\title{
Crossing borders: theory, assessment and treatment in borderline personality disorder
}

Citation for published version (APA):

Giesen-Bloo, J. H. (2006). Crossing borders: theory, assessment and treatment in borderline personality disorder. [Doctoral Thesis, Maastricht University]. Datawyse / Universitaire Pers Maastricht. https://doi.org/10.26481/dis.20060908jg

Document status and date:

Published: 01/01/2006

DOI:

10.26481/dis.20060908jg

Document Version:

Publisher's PDF, also known as Version of record

\section{Please check the document version of this publication:}

- A submitted manuscript is the version of the article upon submission and before peer-review. There can be important differences between the submitted version and the official published version of record.

People interested in the research are advised to contact the author for the final version of the publication, or visit the DOI to the publisher's website.

- The final author version and the galley proof are versions of the publication after peer review.

- The final published version features the final layout of the paper including the volume, issue and page numbers.

Link to publication

\footnotetext{
General rights rights.

- You may freely distribute the URL identifying the publication in the public portal. please follow below link for the End User Agreement:

www.umlib.nl/taverne-license

Take down policy

If you believe that this document breaches copyright please contact us at:

repository@maastrichtuniversity.nl

providing details and we will investigate your claim.
}

Copyright and moral rights for the publications made accessible in the public portal are retained by the authors and/or other copyright owners and it is a condition of accessing publications that users recognise and abide by the legal requirements associated with these

- Users may download and print one copy of any publication from the public portal for the purpose of private study or research.

- You may not further distribute the material or use it for any profit-making activity or commercial gain

If the publication is distributed under the terms of Article $25 \mathrm{fa}$ of the Dutch Copyright Act, indicated by the "Taverne" license above, 


\section{Crossing Borders:}

Theory, Assessment and Treatment in Borderline Personality Disorder 
Cover design by Rob Klein Goldewijk

Lay-out by Ine Kengen

(c) J.H. Giesen-Bloo, Maastricht 2006

ISBN-10: 9052785511

ISBN-13: 9789052785516

Published by Universitaire Pers Maastricht

Printed in the Netherlands by Datawyse Maastricht 


\title{
Crossing Borders: Theory, Assessment and Treatment in Borderline Personality Disorder
}

\author{
Proefschrift \\ ter verkrijging van de graad van doctor aan de Universiteit Maastricht, \\ op gezag van de Rector Magnificus, Prof. Mr. G.P.M.F. Mols, \\ volgens het besluit van het College van Decanen, \\ in het openbaar te verdedigen op vrijdag 8 september 2006 om 14.00 uur \\ door \\ Josephine Hubertine Giesen-Bloo \\ Geboren op 6 augustus 1971 te Nijmegen
}

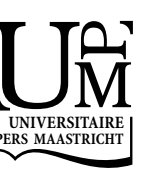




\section{Promotores}

Prof. dr. A. Arntz

Prof. dr. R. van Dyck (VU Medisch Centrum)

Prof. dr. P. Spinhoven (Universiteit Leiden)

\section{Beoordelingscommissie}

Prof. dr. A. Jansen (voorzitter)

Dr. D. Bernstein

Prof. dr. W. van den Brink (Universiteit van Amsterdam)

Prof. dr. J. Livesley (University of British Columbia)

Prof. dr. J. Vlaeyen

This research was supported by Grant 0G-97.002

of the Dutch Health Care Insuranceboard 
Hier sta ik,

ook voor mw. mr. L.M. Bloo-Rosmalen

voor pappa 



\section{Contents}

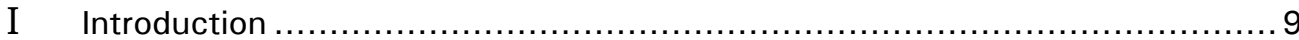

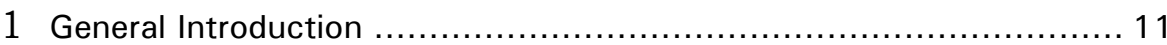

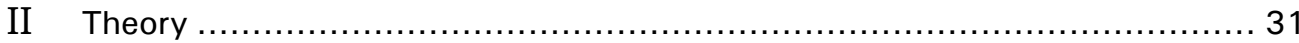

2 World Assumptions and the Role of Trauma in Borderline Personality Disorder ............................................................. 33

3 The Defense Style Questionnaire-48: Factor structure in clinical and non-clinical samples................................................... 47

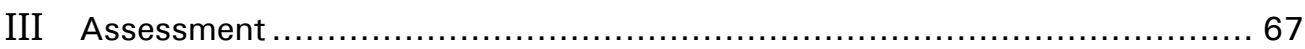

4 Assessment of Borderline Personality Disorder with the Borderline Personality Disorder Severity Index-IV: psychometric evaluation and dimensional structure ........................69

5 The Borderline Personality Disorder Checklist: Psychometric evaluation and factorial structure in clinical and nonclinical

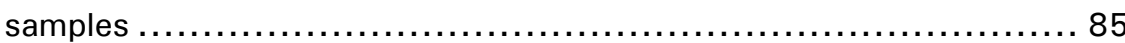

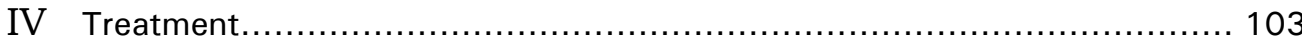

6 Outpatient Psychotherapy for Borderline Personality Disorder: A randomized trial of Schema focused therapy versus Transference focused psychotherapy

7 One-Year follow-up of Outpatient Psychotherapy for Borderline Personality Disorder: Schema focused therapy versus Transference focused psychotherapy

8 The Therapeutic Alliance in Schema Focused Therapy and Transference Focused Psychotherapy for Borderline Personality Disorder

V Summary and General Discussion ................................................. 163

9 Summary and General Discussion ....................................... 165

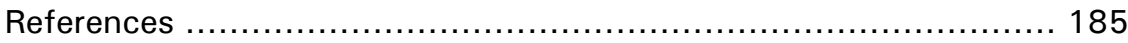

Dutch Summary / Nederlandse samenvatting ............................ 201

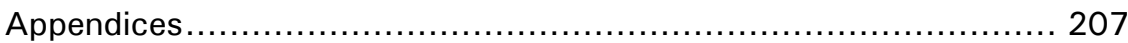

I Interpersonal behavior style of therapists ..................... 209

II BPDSI-IV Criterion cutoff scores ............................... 211

III DSQ-48 (en 43) ............................................... 213

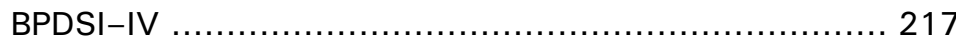

BPD checklist/klachtenlijst ............................... 235

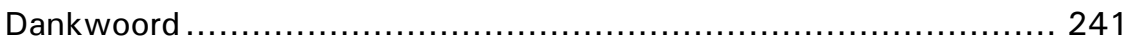

Curriculum vitae ......................................................... 245 



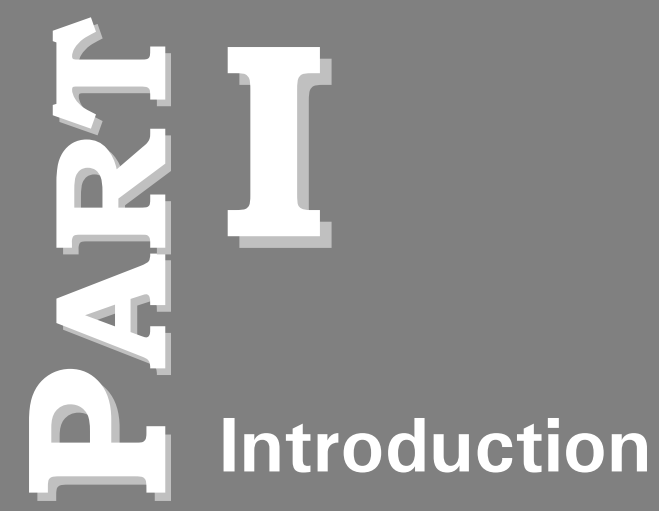




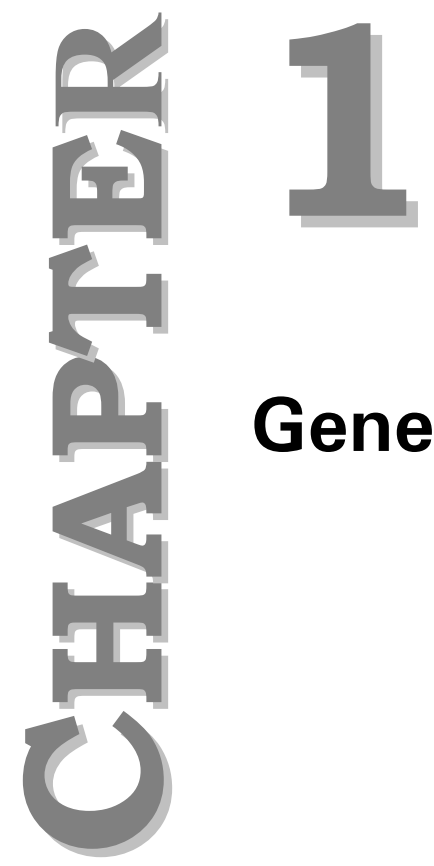

\section{General Introduction}

\section{Adapted from:}

Giesen-Bloo, J. \& Arntz, A. (2000). Borderline persoonlijkheidsstoornis: een uitdaging voor behandeling (Borderline personality disorder: a challenge for treatment). De Psycholoog, 35, 317-324.

and

Giesen-Bloo, J. \& Arntz, A. (2000). Psychoanalytische psychotherapie en cognitieve gedragstherapie voor borderline persoonlijkheidsstoornis (Psychoanalytical psychotherapy and cognitive behavioral therapy for borderline personality disorder). Gedrag \& Gezondheid, 28, 288-295. 



\section{Introduction}

Diana is 32-year old, married, housewife and mother of a toddler. She is admitted to the psychiatric ward of an academic hospital because of a crisis and an unbearable home situation: she threatened her husband with a knife, ventilated her frequent angry moods mostly by smashing crockery and speeding, and tried to overcome the subsequent feelings of emptiness by cutting in her arms or taking lots of valium. Diana felt that she could not hold any responsibility for her behavior anymore, because she experienced a lack of control over her temper tantrums. Her husband also could not deal with the situation anymore. The immediate cause to the current crisis is yet not completely clear. For years, Diana had no contact with her father. However, bringing up his death triggers all kinds of feelings that are difficult to cope with. The attending psychologist suspects a history of sexual abuse but does not pursue the topic out of fear for further decompensation. Diana does not know her husband for a long time, and she is convinced that he will leave her. Her child stems from a previous short-lived relationship. Before getting married, Diana regularly threw herself in passionate relationships, after which she broke them up as abruptly as they started, due to her fear of abandonment. During this period she also misused different drugs and alcohol. Diana made several suicide attempts, some seemed halfhearted: taking many but surely not enough pills, suddenly crossing busy roads etcetera. After graduating from high school, she enrolled in several courses, however she did not finish any of these. Once pregnant, she thought that being a housewife and mom was "her destiny". Nevertheless she now experiences her child as restricting her life too much.

The above case of someone with a Borderline Personality Disorder (BPD), illustrates the complexity of the problems BPD-patients often experience. BPD cannot only be difficult for the individual suffering from it, but also for people around him/her (whether they are partners, family, friends or colleagues), as well as for people who come across BPD-individuals because of their work in (mental) health care settings or other social services. The many different aspects of BPD have consequences for the treatment possibilities. This in addition to the relative short existence of BPD as diagnosis. Therefore, most of the common existing treatments cannot offer adequate solutions to the complexity of BPDpsychopathology. At times therapists feel that their treatment of BPD-patients is as to fight a losing battle, due to a lacking suitable treatment plan. Therapists regularly experience feelings of frustration and incompetence towards BPDpatients. Next to these observations, it is striking that, as noted by Arntz (2005) "there is probably no other mental disorder about which so much has been written as BPD while at the same time so little is known from empirical research as BPD". 
BPD is the central focus of this dissertation, in which several studies with different scopes are compiled. In this introductory part the conceptual development, characteristics and etiology of BPD are described. Furthermore, attention is given to the state of affairs with respect to BPD-treatment and assessment. An outline of the dissertation's aims concludes this chapter.

\section{BPD Conceptualization}

\section{Historical development}

For years, 'borderline' has been used with different interpretations, thereby referring to different groups of patients. In 1938 Stern was the first to give a clinical description of a 'borderline' patient (Stern, 1938). He wrote on a heterogeneous group of patients who had no to little benefit from classical psychoanalytic treatment and besides that, did not fit in the neurotic and psychotic classifications of that time. 'Borderline' indicated patients whose problems lied between the neurotic and psychotic psychopathology. Following this, the 'borderline' concept became popular, especially in psychoanalytic circles. Nowadays, the 'borderline' concepts of Kernberg, Gunderson and the Diagnostic and Statistical Manual of Mental Disorders (DSM) are the most influential.

The psychodynamically oriented view of (Kernberg, 1984; Kernberg, 1996) describes the organization of personality using three criteria that determine someone's ego structure. These are the level of identity integration, the quality of defensive operations and the intactness of reality testing. The patient with a borderline personality organization is dealing with a lack of identity integration (or in other words, with identity diffusion) and is mainly relying on primitive defense mechanisms in which 'splitting' takes a prominent position. Splitting is characterized by a total emotional disconnection between contradictory affect states and a failure to integrate the positive and negative qualities of the self or others into cohesive images. Ambivalent affects cannot be experienced simultaneously. For example, BPD-patients tend to alternate between polar opposites, they experience somebody else and/or oneself as totally good or as a totally bad person (Clarkin, Yeomans, \& Kernberg, 1999). The reality testing is in general in tact. Kernberg's criteria can be assessed by the psychoanalytically trained interviewer with a Dutch version of the 'structured interview' (Derksen, Hummelen, \& Bouwens, 1988).

Kernberg's borderline conceptualization relegates to a person's intrapsychic functioning, which is in contrast to the BPD-concept of Gunderson and the DSM that refer to manifest behaviors and experiences. Gunderson developed his criteria after reviewing the clinical literature and operationalized these criteria in the 
Diagnostic Interview for Borderline patients (DIB; Gunderson \& Singer, 1975). Further research led to a revision of both the criteria and interview (Derksen, 1988; Gunderson \& Zanarini, 1987; Zanarini, Gunderson, Frankenburg, et al., 1989). The borderline personality disorder as such was first defined in the DSMIII (APA, 1980). Eight criteria were formulated after research of (Spitzer, Endicott, \& Gibbon, 1979). The release of the DSM-III-R (1987) hardly held any changes to the BPD criteria. An important difference came with the DSM-IV (1994), a ninth criterion was added concerning short episodes of lost reality testing. Semi-structured interviews, like the 'Structured Clinical Interview for DSMIV of axis II' (SCID-II; First, Gibbon, Spitzer, et al., 1997; Weertman, Arntz, \& Kerkhofs, 2000) and the Structured Interview for Personality Disorders' (SIPD; de Jong, Derks, van Oel, et al., 1996; Pfohl, Blum, \& Zimmerman, 1997) can be used to assess the DSM-based borderline definition.

Tabel 1. DSM-IV-TR Diagnostic Criteria for Borderline Personality Disorder A pervasive pattern of instability of interpersonal relationships, self-image, and affects, and marked impulsivity beginning in early adulthood and present in a variety of contexts, as indicated by five (or more) of the following:

1. Frantic efforts to avoid real or imagined abandonment. Note: Do not include suicidal or self-mutilating behavior covered in Criterion 5 .

2. A pattern of unstable and intense interpersonal relationships characterized by alternating between extremes of idealization and devaluation.

3. Identity disturbance: markedly and persistently unstable self-image or sense of self.

4. Impulsivity in at least two areas that are potentially self-damaging (e.g., spending, sex, substance abuse, reckless driving, binge eating). Note: Do not include suicidal behavior, gestures or threats, covered in Criterion 5.

5. Recurrent suicidal behavior, gestures, or threats, or self-mutilating behavior.

6. Affective instability due to a marked reactivity of mood (e.g., intense episodic dysphoria, irritability, or anxiety usually lasting a few hours and only rarely more than a few days).

7. Chronic feelings of emptiness.

8. Inappropriate, intense anger or difficulty controlling anger (e.g., frequent displays of temper, constant anger, recurrent physical fights).

9. Transient, stress-related paranoid ideation or severe dissociative symptoms.

Note. From American Psychiatric Association (2000, p. 710).

In clinical practice, patient groups diagnosed with the Gunderson borderline concept or with the DSM-III/-R borderline concept are almost identical (Skodol \& Oldham, 1991). Inherently to Kernberg's structural approach of personality organization most patients with a DSM-IV personality disorder, but also many patients with a DSM-IV axis-I disorder, have a borderline personality organization (Kernberg, 1996; Kernberg, Selzer, Koenigsberg, et al., 1989). The patient group 
diagnosed with Kernberg's method is considerably larger and has therefore moderate correspondence with groups diagnosed according to Gunderson or the DSM-III(-R) (Kullgren \& Armelius, 1990).

The conceptualization of the borderline personality disorder has led to an enormous increase in empirical research of this disorder. For example, a review of (Zimmerman, 1994) shows that in particular the reliability and validity of the DSM-concept have been studied intensely since the 1980's. The DSM-criteria for BPD are further widely used in clinical practice and in BPD related research, also by psychodynamical oriented research groups like Kernberg's group. Therefore, in this thesis 'BPD' further indicates the DSM borderline concept. The DSM-IV-TR diagnostic criteria for BPD can be found in Table 1.

\section{Current issues in BPD conceptualization}

Even though the DSM-IV conceptualization of BPD is widespread in clinical practice and in research, the debate on this concept's validity is very much alive. Commonly cited concerns with respect to the categorical DSM model of personality disorders (including BPD) are the excessive diagnostic co-occurrence, heterogeneity among persons with the same diagnosis, absence of non-arbitrary boundary with normal functioning, and inadequate coverage of maladaptive personality functioning (Widiger \& Mullins-Sweat, 2005). Opinions that the BPDconcept should be closely linked to either post traumatic stress disorders, affective disorders or schizophrenia have largely passed (Akiskal, Chen, Davis, et al., 1985; Gunderson \& Phillips, 1991; Gunderson \& Sabo, 1993). Based on the DSM criteria for BPD, there are several studies that examined if differentiations within a (borderline) personality diagnosis or integrations with dimensional profiles could possibly lead to better manageable concepts and understanding (e.g. Hare, Hart, \& Harpur, 1991; Oldham, Skodol, Kellman, et al., 1995; Tyrer \& Johnson, 1996). Suggestions to position a (borderline) personality disorder, together with axis-I disorders, on the continuum of general personality function, have also been made, for instance within the five-factor-model (Lynam \& Widiger, 2001; Trull, Widiger, Lynam, et al., 2003), the interpersonal circumplex (Benjamin, 1996; Kiesler, 1996) and the seven-factor model (Cloninger, 2000). Findings with respect to the presence of higher-order domains within the BPDdiagnostic criteria are mixed. Several proposals for three- and four factor BPDmodels have been made by statistically reducing DSM based BPD-criteria into factors or clusters (Clarkin, Hull, \& Hurt, 1993; Hurt, Clarkin, Widiger, et al., 1990; Livesley \& Schroeder, 1991; Sanislow, Grilo, \& McGlashan, 2000; Sanislow, Grilo, Morey, et al., 2002). The most recent study (Sanislow, Grilo, et al., 2002) found empirical evidence for three homogeneous components: disturbed relatedness, behavioral dysregulation, and affective dysregulation. Yet, other re- 
sults argue for a one-dimensional view of the DSM BPD-criteria over higher-order factors (Arntz, 1999; Fossati, Maffei, Bagnato, et al., 1999).

One of the aims of the present thesis is to contribute to the conceptual debate on BPD. Two studies, which will be presented in Part III, examine whether BPD as a one, a higher order or a DSM-based multidimensional concept is supported best.

\section{Characteristics of BPD patients}

About $1.5-2 \%$ of the general population, $10 \%$ of the outpatient, and $20 \%$ of the inpatient clinical psychiatric patients has a BPD-diagnosis (APA, 1994; Ingenhoven \& van den Brink, 1994; Linehan, 1993b). Of the psychiatric patients being treated for a personality disorder, 30 to $60 \%$ have this diagnosis (APA, 1994). The incidence is unknown but, based on the prevalence and the disorder's duration, can be estimated at 50 new patients per 100.000 persons per year in the age category of 18 to 65 years. Predominance of the BPDdiagnosis in women with an estimate of $75 \%$ is mostly found in clinical settings (Morey, Alexander, \& Boggs, 2005). However, non-clinical epidemiological community studies reveal no gender differences (Torgersen, Kringlen, \& Cramer, 2001; Zimmerman \& Coryell, 1990).

From a pragmatic point of view, the most notorious / well-known symptoms of BPD are recurring crises, hospitalizations, automutilation, suicide attempts, addiction, and depressive/ anxious/ aggressive episodes. About $9 \%$ of BPD-patients, up to $36 \%$ of the most severe BPD-patients, commits and succeeds in their suicide attempt (APA, 1994; Linehan, 1993a; Paris \& ZweigFrank, 2001). Traditional insight oriented psychotherapy does not reduce the suicide risk: an average of $10 \%$ was found in four long-term follow-up studies (Paris, 1996).

Comorbidity with other psychiatric disorders is substantial. BPD-patients often fulfill to criteria of one or more other DSM axis-I and/or axis-II disorder. Most common is the axis-I co-occurance of mood disorders, anxiety disorders, substance use disorders and eating disorders. These comorbid disorders can alternate in a relative high pace, while the BPD-pathology remains present as a stable characteristic. This is often viewed as an inherent aspect of BPD: the severe psychopathology affects the proneness for developing other psychiatric problems. For axis-II, odd, anxious and dramatic cluster personality disorders are each common among BPD-patients. However, the relationship between anxious cluster personality disorders and BPD is particularly strong (Ingenhoven \& van den Brink, 1994; Widiger \& Frances, 1989; Zanarini, Frankenburg, Dubo, et al., $1998 \mathrm{a} ; \mathrm{b})$. 
BPD-patients often experience difficulties in maintaining and keeping up with their work or education, and this is equally so with respect to intimate relationships. These features contribute to the relatively low quality of life that BPDpatients experience (Torgersen, Kringlen, et al., 2001). BPD-patients further have high levels of societal dysfunction and disability (Skodol, Gunderson, McGlashan, et al., 2002). BPD-symptoms tend to calm down in middle age/ late middle age, even though people continue to experience clinical significant problems and quality of life does not appear to improve with ageing (Paris, 1996; Paris \& ZweigFrank, 2001). Typical for BPD-patients, especially in comparison with other personality disordered patients, is that they frequently use both mental and somatic health care facilities, contributing to enormous societal costs (APA, 1994; Chiesa, Fonagy, Holmes, et al., 2002; Linehan \& Heard, 1999; van Asselt, Dirksen, Severens, et al., 2006; Zanarini, Frankenburg, Hennen, et al., 2004). Based on the known mental health care costs in the Netherlands and the BPD-prevalence of the early 1990's, it could be estimated that the annual (governmental) spending on BPD-patients (e.g. through Disablement Insurance Act, Dutch Health Law, unemployment) is at least 341 million euros (750 million Dutch guilders; StatenGeneraal, vergaderjaar 1995-1996; Ten Have, Lorsheyd, Bijl, et al., 1995).

\section{Etiology of BPD}

At present, the etiology of BPD is generally considered to fit a biopsychosocial model, in which biological, psychological and social factors interact in multiple ways in the development and further course of BPD. The basic argument for this model is that although biological, psychological, and social factors are all necessary to develop BPD, none of them by themselves are sufficient to result in a BPD-diagnosis (Paris, 1996).

Biological factors in BPD cover heritability of pronounced presence of personality dimensions (e.g. temper) and personality traits (e.g. extraversion, emotional stability or impulsivity), possible dysfunction of the emotion-regulation system, deviating neurotransmitter levels (i.c. serotonin, noradrenalin and acetylcholine), and dysfunction in (many possible) brain structures related to inhibition and emotional informational processing (Adams, Bernat, \& Luscher, 2001; Coccaro \& Siever, 2005; Linehan, 1993a; Nahas, Molnar, \& George, 2005; Skodol, Siever, Livesley, et al., 2002).

Psychological factors consist of childhood trauma, disorganized attachment system, non-optimal information processing, and etiological theories of different psychological orientations. Early childhood trauma, caregiver neglect, and disorganized attachment style are common in histories of BPD-patients and are significant risk factors in the development of BPD (e.g. Arntz, 2004; Fonagy, 2000; 
Sabo, 1997). According to psychoanalytic theory, BPD originates in a combination of (biological) predispositional factors and disturbances in the early relationships with the caregivers. It is believed by modern psychoanalytical theorists (guided by the object-relation theory) that developmental disorders like BPD, are mainly due to a problematic separation-individuation phase, and shortcomings in emotional responses of the mother. Because of this, the borderline patient mainly uses primitive defenses (over neurotic and mature defenses), is very weak in impulse regulation, and can insufficiently tolerate anxiety. The patient can hardly integrate contradictory feelings and thoughts of his/her self and of others, and therefore these parts are split-off. Complaints and symptoms are read as expressions of underlying conflicts, that are mostly unconscious. (Kernberg, 1984; Kernberg, 1996). Cognitive theorists stress the role of assumptions, hypervigilance, dichotomous thinking and poor sense of self (Arntz, 1999; 2004; Beck, Freeman, \& Associates, 1990; Layden, Newman, Freeman, et al., 1993). The three key assumptions in BPD are: (1) The world is dangerous and malevolent, (2) I am powerless and vulnerable, and (3) I am inherently unacceptable. Another more behavioral view, in which dialectical principles and zen-mindfulness are emphasized, see BPD as a consequence of a dysregulation of emotional control (Linehan, 1993b). This dysregulation is attributed to an interaction between genetically determined emotional vulnerability and an emotionally invalidating childhood environment.

The presence and factorial structure of defense mechanisms as part of Kernberg's psychoanalytical theory and the key assumptions of Beck's cognitive theory were tested in this thesis' context. These studies are presented in Part II.

Social factors refer to environmental influences. Even though these aspects are less investigated than biological and psychological factors, some social factors are found likely to contribute to BPD-development. Living in chaotic and unpredictable family environments, the breakdown of traditional structures resulting in lower social cohesion, fluid versus fixed social roles, and lower continuity between generations-, as well as the rising demand on people to determine individual moral values for autonomous functioning due the (high) pace of societal changes, are named as potential risk factors (Millon \& Grossman, 2005; Paris, 1996; 2005). BPD-patients whose instability actually demands living in a secure and supporting environment, are obviously not protected or helped by the mentioned societal developments.

\section{Assessment in BPD}

Accurate assessment of BPD, whether for diagnostic or research purposes, is essential for both clinical and theoretical comprehension of BPD-pathology. 
This is in fact true for all disorders. However, when insufficient attention is given to the properties and validity of assessment instruments, the result will lead to a blurred perception instead of an increased understanding of a given disorder. This can be traced to the observation that the principles underlying the classification of BPD -the concept's validity- are also deduced from the manner in which BPD is assessed, not only by interpreting data that were obtained with an assessment instrument. Therefore, assessment can affect a concept's validity and, in turn, its theoretical and pragmatic meaning. The other way around, different types of assessment instruments are developed and validated after specific (BPD-)concepts. In that case, the assumption is held that the current concept already has adequate validity to serve as starting point. Following this, it should further be stated that validity can only be established when an assessment method is also deemed reliable (Zimmerman, 1994).

The choice of assessment method often depends on it's purpose (e.g. clinical versus research setting, use in different theoretical orientations) and practical possibilities (e.g. time investments, personnel and costs). Structured and semistructured interviews, and self-administered questionnaires are most common. The available instruments for assessment of (parts of) personality disorders in general are countless (McDermut \& Zimmerman, 2005). The most widely used and prominent diagnostic interviews for personality disorders (Structured Interview Kernberg, DIB-R, SCID-II, SIDP-IV) are already mentioned in the section on BPD-conceptualization. Diagnostic self-report questionnaires tend to overdiagnose and are therefore more suitable for screening aims. However, the nature and complexity of BPD also require specific assessment instruments to facilitate both clinical and research purposes, as some of the current methods encounter interpretative or practical problems with BPD-patients. For example, diagnostic interviews are presently often used as outcome measure in treatment outcome studies, even though they are not designed to detect change, whereas many self-report questionnaires only inquire into certain aspects of BPD instead of the whole array of possible BPD-symptoms.

Another focus of this thesis is further development of BPD-specific assessment instruments: the BPD Checklist self-report questionnaire and the Borderline Personality Disorder Severity Index - IV (BPDSI-IV) semi-structured interview, which are both presumed to assess the severity and frequency of BPD-specific psychopathology in great detail, and to detect changes due to treatment. The BPD Checklist reflects the experienced burden of the pathology by the patient. The BPDSI-IV provides more objective observer-rated information. These studies will be presented in part III. 


\section{State of affairs in BPD treatment}

\section{Crisis and medication}

BPD-patients mostly turn to health care facilities at times of crises (e.g., suicide attempts / threats, despair, substance abuse, automutilation; Turner, Becker, \& DeLoach, 1994). Crisis interventions are of course imperative, but are not sufficient; for that long-term treatment is essential. An accompanying issue is that it is not always clear what needs to be treated; the personality disorder itself, a cluster of symptoms within a personality disorder and/or associated axisI disorders (Gitlin, 1993). What can be said is that medication targeting affective and anxious psychopathology as part of the BPD, can offer (temporary) relief. Pharmacotherapy may also allow BPD-patients to reflect before acting, indirectly providing an entrance for psychosocial interventions and with that the possibility to discontinue medication once patients have learned to manage themselves. But again, pharmacotherapy as it is, does not present an adequate treatment of BPD. (Layden, Newman, et al., 1993; Lieb, Zanarini, Schmahl, et al., 2004; Paris, 1996). Therefore, medication is frequently used with some other treatment modality to relief the acute symptomatic suffering. Combining psychosocial treatment and pharmacotherapy is not easy. The mere possible misuse of prescribed medication by BPD-patients is a realistic risk during treatment (Waldinger \& Frank, 1989). A recent study even suggests that pharmacotherapy may negatively interact with psychological treatment (Simpson, Yen, Costello, et al., 2004).

\section{Process and therapist-patient aspects}

Even though the American Psychiatric Association has published practice guidelines for the treatment of BPD (Oldham, Gabbard, Goin, et al., 2001), it is not yet possible to distinguish a predominant standard treatment within the wide range of psychiatric care available. The current APA-guidelines received severe criticism, and above all demonstrated the need for research in the different aspects of BPD (e.g., McGlashan, 2002; Paris, 2002; Sanderson, Swenson, \& Bohus, 2002; Tyrer, 2002). The difficulty in finding acceptable and clear BPDtreatment possibilities can in part be explained by the heterogeneity of the disorder; each individual BPD-patient needs, to some degree, a tailor-made treatment. A number of factors determine the treatment setting(s), of which the patient's baseline psychopathology, the capacity to engage in a therapeutic relationship and the development during treatment are illustrative (Derksen, Bender, \& Roverts, 1994). The latter authors reviewed several studies on treatment effectiveness for BPD-patients, and identified five factors that are of influence on the 
prosperity of a treatment: (1) quality of the therapeutic relationship, (2) a stable therapeutic frame for the protection of unpredictable and often destructive behavior of the patient, (3) the psychotherapeutic interventions, (4) the treatment's management, especially for intramural settings and (5) pharmacotherapy. With respect to 'psychotherapeutic interventions', it should be noted that the reviewed studies did not agree on which type of interventions contributes to effectiveness. Moreover, the effectiveness of interventions is, in part, dependent of the therapist-patient relationship.

The quality of the therapist-patient relationship, the therapeutic alliance, is generally a robust predictor of treatment outcome (e.g., Horvath \& Symonds, 1991; Martin, Garske, \& Davis, 2000). The importance of the therapist's contribution with respect to interpersonal behavior in the therapy process, next to that of a patient, is also demonstrated by poor treatment outcome when a therapist is (unintentionally) 'drawn' towards negative enmeshment (Henry, Schacht, \& Strupp, 1990). Many treatment manuals carefully 'prescribe' and elaborate on the attitude/stance and demeanor therapists should take on within it's paradigm to achieve successful working relationships and treatment outcomes. This is specifically relevant for BPD-treatments since difficulties in establishing and maintaining (intimate) relationships is so characteristic of BPD-pathology. Constant attention of the therapist on developing a collaborative, constructive therapist-patient relationship, as well as monitoring this relationship during the course of a BPD-treatment, therefore appears a prerequisite. Apparently, it is not selfevident for therapists to note disruptions or threats in a therapeutic alliance that are negatively influencing the patient's change process (Regan \& Hill, 1992; Safran, Muran, Samstag, et al., 2001). Taken together with the potential ability to (in part) successfully identify patients that are high-risk for drop-out by means of the therapeutic alliance and interpersonal behavior styles, these findings underline how essential the working relationship between patient and therapist for positive treatment outcome is (Samstag, Batchelder, Muran, et al., 1998).

\section{Current treatments of BPD}

The prevailing clinical impression is, and fortunately nowadays also for many professionals that already changed to was, that in most mental health care settings no serious efforts for BPD-treatment are made. For instance, the BPDpatient is rapidly discharged or sent away, or only cursory counseled, leaving the actual psychopathology unchanged. Low frequent and long-term treatments (e.g. in social psychiatric service departments of community mental health centers and hospital's outpatient clinics), not aiming for change but just (temporary) stability in the patient, seems to be offered most frequently. Of the available, described BPD- treatments, clinical practitioners often apply psychoanalytically ori- 
ented treatments in both intramural and extramural settings, although the popularity of dialectical behavior therapy is rapidly rising (Linehan, 1993a). The mutual differences in psychodynamic treatment application are large, whether or not caused by the fact that one treatment uses elements of another school's treatment. Some examples of current psychoanalytically based treatments are expressive-supportive therapy, transference-focused psychotherapy, processfocused psychotherapy, mentalization based treatment, group therapy, conversational therapy, private practice counseling and partial hospitalization (Bateman \& Fonagy, 1999; 2004; Clarkin, Yeomans, et al., 1999; Derksen \& Groen, 1994; Kernberg, Selzer, et al., 1989; Luborsky, 1984; Meares, 2004; Rockland, 1992). The definition of BPD in the DSM-III provided leads for other theoretical schools within the psychotherapeutic community, to develop 'their own' specific BPDtreatment. Illustrative of manualized treatments with different theoretical roots are cognitive therapies for BPD (Arntz, 2004; Layden, Newman, et al., 1993; Pretzer, 1990), schema focused cognitive behavioral therapy (Young \& Klosko, 1994; Young, Klosko, \& Weishaar, 2003), interpersonal and cognitive behavioral group therapy (Blum, Pfohl, John, et al., 2002; Marziali \& Munroe-Blum, 1994), and cognitive analytic therapy (Ryle, 1997).

As mentioned before, research on BPD increased dramatically with the publishing of the DSM-III. Nevertheless, effectiveness of BPD-treatments remained untested for many years. Studies that were performed often failed methodological standards. Paris' report (1996) on four large-scale long-term follow-up studies, in which intensive inpatient psychoanalytical therapy was investigated, is not very positive. The principal conclusions indicate that: there is little improvement in the short term, recovery only becomes apparent after ten years of treatment, patients continue to have serious problems during their lives, and the suicidal risk remains high. On the other hand, one report of two years of inpatient psychoanalytical treatment give a more optimistic impression (Tucker, Bauer, Wagner, et al., 1987). BPD-patients improved with respect to impulsive behavior, social adjustment, and number of hospitalizations. Psychoanalytical studies in outpatient settings demonstrate positive treatment effects after one year (Clarkin, Foelsch, Levy, et al., 2001; Clarkin, Koenigsberg, Yeomans, et al., 1994; Stevenson \& Meares, 1992; Waldinger \& Gunderson, 1984). However, a difficulty with psychoanalytical treatments is the high percentage $(50-67 \%)$ of patients terminating therapy prematurely (Skodol, Buckley, \& Charles, 1983; Waldinger \& Gunderson, 1984; Yeomans, Selzer, \& Clarkin, 1993). Furthermore, none of the referred studies had a controlled design, so results should be interpreted with caution. Other manualized treatments similarly lack valid empirical evidence for their effectiveness. Some case-studies have been documented with promising results (Arntz, 1994a; b; Layden, Newman, et al., 1993; Morrison, 2000; Nordahl \& Nysaeter, 2005; Turner, 1989), like some open trials (Arntz, 
2004; Blum, Pfohl, et al., 2002; Brown, Newman, Charlesworth, et al., 2004; Ryle \& Golynkina, 2000; Wildgoose, Clarke, \& Waller, 2001).

\section{Evidence-based treatments}

Marsha Linehan and her colleagues were the first to conduct a randomized controlled clinical trial, in which dialectical behavioral therapy (DBT) for severe BPD-patients was compared with treatment-as-usual (1991, 1993a, 1993b, 1993c, 1994). DBT was more effective than treatment-as-usual with respect to reduced suicide attempts and automutilation, hospitalizations and treatment fidelity (fewer dropouts). Subjective reports of depression, hopelessness, reasons for living, and suicide ideation did not favor DBT over treatment-as-usual. The positive findings were maintained at 1 -year follow-up. Treatment-as-usual was not defined, and therefore not limited to psychodynamically based treatments. The DBT-trial was replicated in the Netherlands with similar findings (Verheul, van den Bosch, Koeter, et al., 2003).

Psychoanalytic long-term partial hospitalization is the only other treatment modality that has shown to be effective in a controlled study (Bateman \& Fonagy, 1999; 2001). BPD-patients in this program were doing significantly better than patients receiving treatment-as-usual. They improved in depressive symptoms, decreased in suicidal and self-mutilatory acts, reduced inpatient days, and showed better social and interpersonal functioning after 6 months of treatment, and continued this development to the end of treatment. Another 18month follow-up demonstrated that effects were not only maintained but also revealed a statistical significant improvement on most measures in contrast to the control patients. However, the results have not been replicated in a second trial nor examined by other independent researchers. Other complications are the differences in treatment intensity between two conditions (e.g partial hospitalization with a whole weekly package of individual psychotherapy, three hours of group psychotherapy, expressive therapy and a community meeting for 1.5 years versus control which existed of two (mean) psychiatric reviews per month, 11.6 (mean; for 90\%) inpatient admission days, with discharge to partial hospitalization (mean 6 months; for $72 \%$ ) followed by outpatient and community follow-up (fortnightly visit of psychiatric nurse; for $100 \%)$ ).

A summarizing remark of the above illustrates that it is possible to bring about (symptomatic) improvement in BPD-patients. Still, the high drop-out rates of psychodynamically based treatments remain a concern, just as the high ongoing suicide risk. Another disadvantage is that often only a portion of the BPDpatients qualifies for certain treatments. With respect to the therapeutic expectations of BPD, cognitive behavioral views tend to hold a relatively more optimistic 
perspective on the possibilities and the pace of change of BPD-manifestations and personality than other theories.

\section{Two experimental treatments for BPD}

Major aim of the current thesis was to determine the effectiveness and relative effectiveness of two promising experimental outpatient treatments for BPD to contribute to a more optimistic perspective of BPD-treatment possibilities. These treatments are psychoanalytically rooted transference focused psychotherapy and cognitive behavioral based schema focused therapy. Both therapies are described with respect to their views on etiology of BPD, objective of treatment, group of borderline patients designed for, key elements, therapy phases and therapist qualifications. Part IV of this thesis presents the actual studies.

\section{Transference Focused Psychotherapy}

Transference Focused Psychotherapy (TFP) is developed by the clinical and research group of Kernberg, thus based on his theory (Clarkin, Yeomans, et al., 1999; Kernberg, Selzer, et al., 1989; Koenigsberg, Kernberg, Stone, et al., 2000; Yeomans, Clarkin, \& Kernberg, 2002; Yeomans, Selzer, \& Clarkin, 1992).

Etiology. Psychoanalytical theory on BPD-etiology is discussed on page 19.

Objective. TFP aims at a better integration of good and bad self-representations in the patient. A second but related goal is to increase the tolerance of the co-existence of good and bad characteristics of others. Reduction in the use of primitive defenses (splitting in particular) and increase of neurotic / mature defenses is a further treatment issue. The transference-relation between therapist and patient is used to realize these objectives.

Aimed borderline patients. TFP is suitable for patients with a borderline personality organization. In DSM-terms, this therapy is appropriate for a large group of axis-II cluster B patients, as well as for patients with a personality disorder NOS. Patients with axis-I pathology can also have a borderline organizational structure, and benefit from TFP. A (comorbid) antisocial personality disorder is contraindicated for TFP, especially when there is an increased level of psychopathy. A treatment history including lack of motivation and dedication to treatment, also leads to an unfavorable TFP-prognosis.

Key elements. The TFP-therapist is much more active in structuring the treatment than in other insight-oriented psychodynamic treatments. The focus in TFP is on the here-and-now rather than on the past (genetic work). The therapist takes on a strictly neutral role - dynamically speaking - in the course of therapy and is not involved in possible crisis interventions outside therapy sessions. Main 
foci of TFP are transference, countertransference, and their interpretation. The transference, on which the therapist concentrates, consists of (primarily aggressively charged) feelings, observations, attitudes and fantasies towards the therapist, that are induced in the therapeutic interaction in the borderline patient. Analyses of this transference brings out the patient's unconscious conflicts so that patient and therapist can work through these conflicts together. Countertransference refers to all emotional responses (thoughts, feelings, fantasies etc.) of the therapist that are experienced in reaction to the patient. Enduring and examining these responses by the therapist is crucial for TFP, since this forms an important information source. Prominent techniques in TFP are exploration, confrontation and interpretation. TFP takes at least two years of sessions twice a week. However, individual differences are considerable.

Therapy phases. TFP has four treatment phases, preceded by a so-called contract phase. The conditions to start TFP are determined in the contract phase. In about six sessions, therapist and patient discuss both their rights and duties until mutually agreed on. Important aim of the contract phase is to protect the treatment against the patient's acting-out. The principal goal of early treatment phase is stabilizing the patient's impulse regulation to reduce acting-out behavior. Starting the development to integrate representations of the patient's self and of others, is the focus of this phase. The therapist will help the patient to understand that his/her fragmented representations function as protection against anxiety, which actually leads to distortions in perceiving day-to-day reality. The midphase is achieved when acting-out behavior is reduced, most potential individual threats of treatment drop-out have subsided, and sort of an equilibrium is reached. A reduction of the number of conflicts in the sessions is also illustrative of the midphase. The dominant tasks of the midphase are to deepen the understanding of the most prevalent transference themes and to deepen the understanding of conflict around primitive aggression and sexuality, as well as their presence in the transference. If the patient can accept and understand the view that his/her identity to consist of separate parts, that he previously rejected by using primitive defense mechanisms, the treatment enters the advanced phase. Further integration of fragmented parts of the patient's self and of others is established in the advanced phase, the integration becomes more complex and realistic. The termination phase arrives once a sufficiently large personality change attained (assessed by the therapist's continuous evaluation), and treatment and life-goals are accomplished. Termination is connected to the entire TFP-process because how a patient accepts termination is a fundamental indication of the general level of functioning. Therefore, possible pathological separation related anxiety towards the therapist (mourning the oncoming 'loss' of the therapist) needs to be addressed, and worked through, during the advanced phase. 
Therapist's qualifications. The principal condition for a TFP-therapist is possessing a thorough knowledge and extensive training in psychodynamical techniques. Clarkin, Yeomans, et al. (1999) aim to develop a treatment which not only is to be performed by psychiatrists, psychologists and psychotherapists, but also by other mental health care professionals like social workers and psychiatric nurses. Regular intervision and supervision are vital to treatment success (especially with respect to possible countertransference influences). In addition, it must be stated that the treatment's quality will (strongly) increase when the therapist has or receives general and/or specific psychoanalytical training.

\section{Schema Focused Therapy}

Beck's early model for cognitive therapy and the constructivist movement inspired Young and colleagues $(1994,1999,2003)$ to develop Schema Focused Therapy (SFT).

Etiology. Central to SFT for personality disorders are schemas. Schemas are knowledge structures which develop during (early) childhood and are expressed in pervasive patterns of thinking, feeling and behaving. Dysfunctional schemas are responsible for the development and maintenance of personality disorders (Arntz \& Kuipers, 1998).

Objective. SFT is an integrative psychotherapy proceeding from a (cognitive) schema model and aims at identifying and changing dysfunctional schemas. Change is achieved through different pathways: cognitive (thinking, reasoning, understanding), experiential (experiencing), and behavioral (acting/behaving).

Aimed borderline patients. SFT, as presented here, is appropriate for DSMIV based BPD patients. However, SFT can also be tailored for patients with axisII cluster B and/or C diagnoses and can be applied for (comorbid) axis-I psychopathology.

Key elements. Young distinguished five schema modes that determine a BPD-patient's state. Schema modes are sets of schemas and coping responses, either unhealthy and/or healthy, which are active at a given moment. A patient can shift from one mode to another, caused by internal or external factors. The five BPD-characteristic modes are the detached protector, the punitive parent, the abandoned/abused child, the angry/impulsive child and the healthy adult. Exact names of the modes are adjusted in consultation with the patient. Furthermore, these names have a metaphorically denotation, no identities or persons are intended. The active mode is continually discussed in treatment sessions, which helps the patient and therapist to understand what is going on. See Arntz \& Kuipers (1998) for a compact review of the specific modes with accompanying schemas, strategies and feelings. SFT has three foci: the therapeutic relationship, life outside sessions, and the past. The therapeutic relationship is extremely 
important to SFT, marked by so-called 'limited reparenting' by the therapist. This implies that the therapist is warm, empathic and stabile, does not reject or abuse the patient, but relates to the patient with respect and setting limits, and acts partly like a "good enough" parent would do towards a small child. The therapist takes on an active role in patient's crises outside sessions. The BPD-patient is even encouraged to contact the therapist in case of a crisis, telephone support is often sufficient. SFT takes at least two years of sessions twice a week.

Therapy phases. SFT has a starting phase and five phases that are related to the five mode model. Some overlap exists between consecutive phases, and sometimes going back to a previous phase can be necessary (e.g., in a crisis). Practical business, a therapeutic contract, basic principles, methods and objectives of the problem conceptualization and of SFT are discussed in the starting phase, lasting between four and eight sessions. The most prominent goal of the first phase is to establish a therapeutic relationship in which the patient perceives the continuous and reliable availability of the therapist. Breaking through 'the detached protector' and 'the punitive parent', as well as a safe attachment of 'the abandoned child' are also important aims. The success of this phase particularly depends of the therapist's behavior in crisis situations. Central to the second phase are symptom- and crises management, meaning that restriction of frequency, intensity and risks for crises, together with the retrieval and discussion of underlying pathology, are then the primary focus. A related point of interest is that crises are not avoided, since the patient needs to experience the therapist as a reliable source of support in these situations. Crises can contribute in accessing difficult aspects of the patient, necessary to reach essential change. Distinctive of the third phase are correcting informational processes, identification of core schemas, and clarifying etiological roots. Therefore, attention is paid to dichotomous thinking, egocentric reasoning, extreme moral values, abandonment and abuse assumptions and feeling inferior. Tackling the 'punitive parent' mode is also emphasized here. Intention of the fourth phase is a definite personality change by means of processing and reinterpreting (traumatic) child experiences, and change of dysfunctional self, other and interpersonal schemas into new adaptive schemas. Furthermore, consolidation of the gained changes is important. Terminating the treatment is the core of the fifth phase, as is further generalization and perpetuation of the changes. Other prominent aspects of this phase are the treatment of rest symptoms, an increase of the patient's independence/autonomy, and the development of healthy (intimate) relationships outside SFT. Actual termination takes place with mutual agreement of therapist and patient.

Therapist's qualifications. As for now, SFT is generally conducted by psychiatrists, psychotherapists, psychologists and social psychiatric nurses, who are 
all extensively trained in Young's model. Like in TFP, regular intervision and supervision and a therapist's clinical experience are vital to an optimal SFT result.

\section{Summary of concrete differences between TFP and SFT}

Significant concrete differences between TFP and SFT can be found in the therapist role, the patient role, and the content of sessions. The therapist in TFP takes on a neutral, reflective stance against a supportive, directive stance of the SFT-therapist. In TFP, the patient agreed to discuss everything that comes up in his/her mind during sessions, and must actively look for help crisis situations other than the TFP-therapist (discussed in contract phase). The patient in SFT is, in contrast, encouraged to contact the therapist in crises. SFT further includes home work assignments and other behavioral techniques that are applicable outside sessions, which are unknown aspects for TFP. Central to TFP-sessions is the discussion of (negatively charged) transferences and their interpretation, whereas in SFT discussions primarily focus on experienced problems between sessions and problematic child experiences.

\section{Thesis' aims and outline}

The present dissertation comprehends seven studies organized in three parts.

Part II contains two studies on theoretical issues in BPD. In Chapter 2 a study is reported that tests the cognitive hypothesis that BPD is characterized by three key assumptions, by using (Janoff-Bulman, 1989) social cognitive 'world assumptive model' of negative effects of trauma. A second aim was to investigate the role of childhood trauma in the content of world views of BPD patients. Chapter 3 focused on the use of defense mechanisms as hypothesized by modern psychoanalytical theorists, by means of the Defense Style Questionnaire (DSQ48). According to Kernberg's object-relation theory different levels of defense mechanisms can be distinguished. BPD-patients are characterized by the use of primitive or immature defense mechanisms (e.g. splitting, idealization/devaluation, primitive denial, projective identification, dissociation and omnipotent control) to protect against intense feelings, affects and impulses that are difficult to accept as part of oneself. Other objectives were to replicate the three-factor structure of the DSQ-36 (Spinhoven, van Gaalen, \& Abraham, 1995), to assess the psychometric properties and to compare the defensive style of different patient and non-patient groups.

Part III consists of two studies on assessment and BPD conceptual issues. 
Chapter 4 and 5 looked into BPD specific assessment instruments: the BPD Checklist self-report questionnaire and the Borderline Personality Disorder Severity Index - IV (BPDSI-IV) semi-structured interview. These instruments were developed to fill a void of BPD-specific instruments, which are required by the nature and complexity of BPD. Both instruments obtain detailed information on the severity and frequency of BPD specific pathology and are designed to function as treatment outcome measure. However, the BPD-Checklist assesses a patient's experienced burden of BPD-symptoms, whereas the BPDSI-IV objectively registers actual BPD-manifestations. Psychometric properties were assessed, clinical norms, specificity and sensitivity derived. Sensitivity to change was only investigated for the BPD-Checklist. However, the sensitivity to change for the BPDSI-IV is illustrated in Chapter 6. Another important objective of these studies involved seven dimensional BPD-models that were used to test whether a one, a higher-order, or a multidimensional BPD-concept is supported best by the BPDChecklist and by the BPDSI-IV data.

Part IV holds three reports of a multicenter randomized controlled trial on outpatient treatment for BPD. Chapter 6 describes the effectiveness and relative effectiveness of Schema Focused Therapy and Transference Focused Psychotherapy during a 3-year course of sessions twice a week, in which patient assessments were three-monthly. Both BPD-treatments' effectiveness in structural change in patients' personality should not only be apparent from a decrease in self-destructive behaviors, but also from reduced pathogenic personality features, reduced general psychopathology, and a consequent increase in quality of life. Chapter 7 examined the maintenance of the found TFP- and SFT-effectiveness after a 1-year follow-up period. Chapter 8 investigated the quality and development of the therapeutic alliance as mediator of change in SFT and TFP.

In Part V, chapter 9, a summary of the preceding study conclusions is provided. Then, results are critically discussed, as is future research.

The designs of studies presented in chapters 4 and 5, like in chapters 6 and 7, are almost identical. Therefore these manuscripts do, in part, overlap each other. 


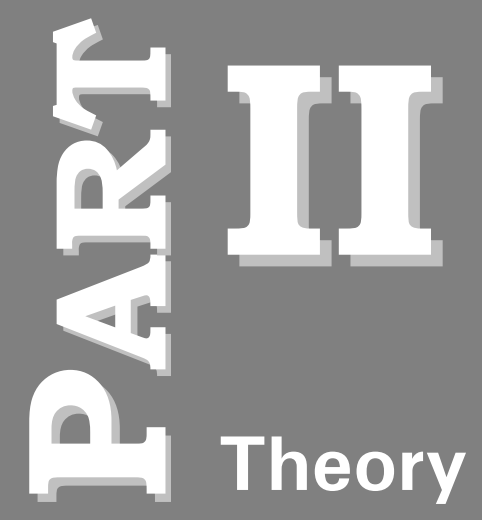





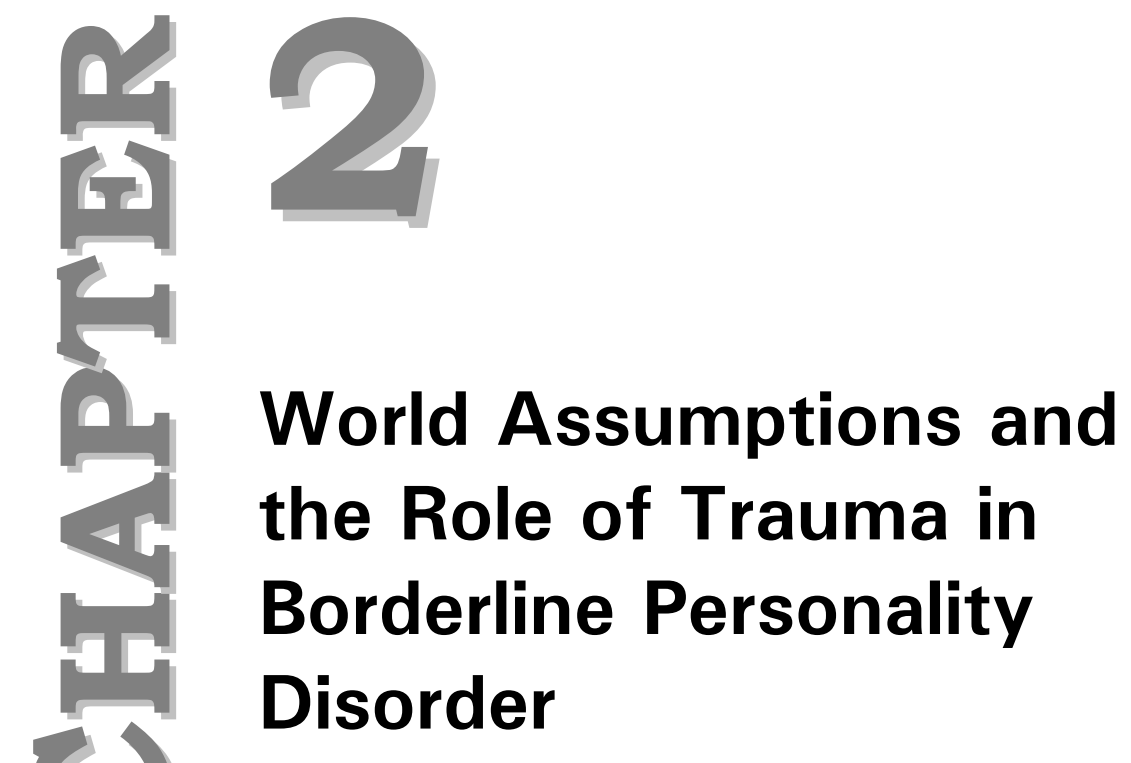

Published as Giesen-Bloo, J., Arntz, A. (2005), Journal of Behavior Therapy and Experimental Psychiatry, 36, 197-208. 


\section{Abstract}

The present study tested whether Borderline Personality Disorder (BPD) is characterized by specific world views as hypothesized by cognitive models, using Janoff-Bulman's world assumptive model of negative effects of trauma (Social Cognition, 1989, 7, 113-136). A second aim of this study was to investigate the role of trauma in the content of world views of BPD patients. Fifteen BPD patients, 14 patients with cluster $C$ personality disorders (PD), 19 patients with axis-I psychopathology and 21 non-patients filled out the World Assumptions Scale, the Personality Disorder Belief Questionnaire, a childhood trauma checklist (assessing physical, emotional and sexual abuse) and the BPD Checklist (severity of BPD psychopathology).

BPD patients appear to view the world as malevolent and perceive less luck independent of trauma but dependent of BPD psychopathology. Furthermore BPD patients have low self worth and persist in specific beliefs independent of trauma or severity of BPD psychopathology. Pretzer's theory of BPD can be largely supported through Janoff-Bulman's world assumptive model. World assumptions of BPD patients can better be explained by the severity of BPD psychopathology than by the presence of trauma. 


\section{Introduction}

The concept of world views or world assumptions was first defined by Parkes, (1971) and Bowlby, (1980). They referred to world assumptions as internal cognitive structures that provide expectations about the world, which enable the individual to develop goals for the future and regulate it's daily functioning. World assumptions are built and solidified over many years. They often go unquestioned because of the individual's need to maintain stability (JanoffBulman, 1992). According to Beck, Freeman, et al., (1990), personality disorders are characterized by specific sets of assumptions. With respect to Borderline personality disorder (BPD), Pretzer, (1990) hypothesized that the basic assumptions focus on three themes: (1) the world is (i.e. others are) dangerous and malevolent, (2) I am powerless and vulnerable and (3) I am inherently unacceptable. Arntz (1994b) and Arntz, Dietzel, \& Dreessen (1999) demonstrated that a set of assumptions lassessed with the personality disorder belief questionnaire (PDBQ)), derived from the work by Pretzer, (1990), were indeed specific for BPD and appeared to be stable. The BPD assumptions seem to constitute an essential characteristic of BPD.

In her work with victims of traumatic events, (Janoff-Bulman, 1989; 1991; 1992) found that most people usually operate on the basic belief of invulnerability. This invulnerability is more or less a derivative from the view that a sense of safety and security is fundamental for the development of a healthy personality and is first developed in early childhood through interaction with the environment. At the basis of personality stands a set of relatively stable fundamental cognitive, on invulnerability based, assumptions about the world en self (the socalled assumptive world). Janoff-Bulman developed a heuristic model specifying the content of these invulnerability-related assumptions. Her model consists of three primary categories, with each category consisting of several assumptions. These categories are (1) perceived benevolence of the world, (2) meaningfulness of the world and (3) worthiness of self.

The first category involves a base rate notion of benevolence/malevolence and is represented by two basic assumptions: the benevolence of the impersonal world and the benevolence of people. The second category, meaningfulness of the world, refers to distributional principles. Assumptions in this category involve the belief that positive and negative events and outcomes are distributed according to the principles of justice (goodness or badness of one's moral character), controllability (engaging in appropriate or inappropriate behaviour) or chance (randomness, meaninglessness). These distributional assumptions do not necessarily exclude each other; people tend to believe in all three principles but to a various extent. Worthiness of self forms the third category and is focusing on the self. The three self-relevant assumptions of this category parallel the men- 
tioned distributional principles namely self-worth, self-controllability and luck. These self-relevant assumptions explicitly deal with the distributional principles on a personal, individual level in contrast with the assumptions of 'meaningfulness of the world'. For example 'chance' assumptions concerns the random distribution of outcomes related to people in general whereas 'luck' regards this aspect to oneself. To explore and compare basic assumptions of victims and nonvictims, Janoff-Bulman (1989) developed the World Assumptions Scale (WAS) made up of eight four-item subscales (6-point Likert scales). She found that people, who experienced a traumatic event many years ago, hold more negative views with respect to perceived self-worth, chance and benevolence of the impersonal world than people who did not. Thus, according to this view, people who experienced negative aftermaths of a trauma have developed negative assumptions regarding perceived self-worth, chance and benevolence of the impersonal world.

The assumptive model of negative effects of trauma of Janoff-Bulman (1989) and the cognitive theory of Pretzer (1990) for BPD do have a striking similarity. BPD's basic theme 'the world is dangerous and malevolent' does fit the negative assumptions in Janoff-Bulman's category of perceived benevolence of the world. 'I am powerless and vulnerable' and 'I am inherently unacceptable' can be related to assumptions of the categories meaningfulness of the world and worthiness of self. More specifically, 'I am powerless and vulnerable' can be placed next to the assumptions of controllability, chance, self-controllability and luck. 'I am inherently unacceptable' is linked with the assumptions of justice and self-worth. Given this, it is possible to test whether Pretzer's basic assumptions are indeed characteristic of BPD by using Janoff-Bulman's theoretical framework. From the above it can be hypothesized that, according to the world assumptive model, BPD individuals view the impersonal world and people as highly malevolent, believe that outcomes are not distributed by the principles of justice, are uncontrollable and determined by chance and do not believe in their self-worth, self-controllability and luck.

A second aspect in this study is the role of trauma in the content of world views. As said before, Janoff-Bulman found differences in world views between victims and non-victims in a sample of undergraduates who were not extensively checked on the presence of psychopathology. Gluhoski \& Wortman (1996) also found significant differences in world views, specifically in perceptions of vulnerability and self-view, for subjects of a community sample who had experienced any type of trauma but weren't screened on the presence of psychopathology. Turning to BPD psychopathology, it is known that a large percentage of BPD patients has experienced childhood trauma, sexually, physically, emotionally or a combination of those (Fossati, Madeddu, \& Maffei, 1999; Herman, Perry, \& van der Kolk, 1989; Sabo, 1997). The findings of Arntz, et al. (1999) support 
the hypothesis that dysfunctional assumptions underlying BPD pathology developed from childhood trauma. This is also to be expected from Janoff-Bulman's theory. To make sure that the BPD patients in the current study have the same specific BPD beliefs previously found (Arntz, Dietzel, et al., 1999; Arntz, Dreessen, Schouten, et al., 2004), and to compare BPD's world views with BPD specific beliefs, the Personality Disorder Belief Questionnaire- BPD section was added. If BPD patients indeed have the hypothesized different world views compared to control groups, the question arises if this is to explain by the high level childhood trauma's BPD patients have experienced or by the severity of BPD psychopathology (checked for with the BPD Checklist) itself.

\section{Method}

\section{Participants}

The patient groups (BPD, Cluster C PD and Axis I psychopathology) were referred by their therapists from the following mental health institutions: the Community Mental Health Center Maastricht, Psychiatric Hospital 'Vijverdal' and Mental Health Center 'Doctor Poelsoord' both in Maastricht, Community Mental Health Center 'Parnassia' in The Hague, Psychiatric Hospital 'Vincent van Gogh' in Venray, Psychiatric Hospital 'St. Jozef' in Bilzen (Belgium), Community Psychiatric Hospital in Rekem (Belgium) and Psychiatric Hospital 'Ziekeren' in St. Truiden (Belgium). Diagnoses were assessed with DSM-IV based semi-structured interviews (SCID I and II). All subjects had to be between age 18 and 60. General exclusion criteria were psychotic disorder and mental retardation. BPD patients were allowed to have comorbid personality and/or axis I disorders. Cluster C patients had to meet criteria for at least one cluster C PD and less than 3 BPD criteria, comorbid axis I psychopathology was allowed. Patients with axis I psychopathology had to meet criteria for at least one axis I disorder and weren't allowed to have threshold diagnoses of PD's or meet any BPD criterion. Nonpatients were recruited with advertisements in free local papers, could not have psychological complaints and could not have any axis I disorder, PD or meet any BPD criterion. After complete description of the study to the subjects, written informed consent was obtained.

\section{Materials}

Dutch versions of the SCID-I and SCID-II were used to diagnose axis I psychopathology and personality disorders (DSM -IV version; First, Gibbon, et al., 
1997; First, Spitzer, Gibbon, et al., 1996; Groenestijn, Akkerhuis, Kupka, et al., 1999; Weertman, Arntz, et al., 2000).

A Dutch version of the WAS (Giesen-Bloo, 2001; Janoff-Bulman, 1989) was used to determine the different sets of assumptions as formulated in the world assumptive model. This self-report questionnaire consists of 32 items which are rated on 6-point Likert scales from strongly disagree to strongly agree. The internal consistencies of the subscales in a student population were satisfactory to good (Cronbach alpha's 0.66 to 0.76; Janoff-Bulman, 1989). The present sample showed moderate to very good internal consistencies (Cronbach alpha's: benevolence of the world 0.88 , benevolence of people 0.71 , justice 0.60 , controllability 0.75 , randomness/chance 0.47 , self worth 0.82 , selfcontrollability 0.77 and luck 0.84 ).

The Personality Disorder Beliefs Questionnaire - BPD section (PDBQ; Arntz, Dietzel, et al., 1999; Arntz, Dreessen, et al., 2004) is developed after Beck's theory of PD (Arntz, 1994b; Beck, Freeman, et al., 1990). It includes 20 beliefs, believed to be specific for BPD. The strength of each belief is rated on $100 \mathrm{~mm}$ visual analogue scales with "I don't believe this at all" on one end and "I believe this completely" on the other end. In a previous study (Arntz, Dietzel, et al., 1999) the PDBQ-BPD section showed an excellent internal consistency (Cronbach alpha 0.95).

A structured childhood trauma interview (Arntz, Dietzel, et al., 1999; van den Bossche, Kremers, Sieswerda, et al., 1999) was used to assess traumatic experiences during childhood. Information was obtained on the occurrence, severity, age(s) of onset, duration, perpetrator(s) and specific acts of sexual abuse, physical abuse and emotional abuse and neglect. The answer categories were pre-set and used to calculate a weighed composite score for childhood trauma, with earlier and more severe, as more related perpetrators, experiences receiving larger weights. The composite trauma score derived from this interview proved to have a good internal consistency (Cronbach alpha 0.80).

The BPD checklist (Arntz, Dreessen, \& Giesen-Bloo, 1999) was added to assess the severity of borderline related symptoms during the last month. Subjects indicated on a 5-point Likert scale from 'not at all' to 'extremely' how much they were troubled by 47 different complaints, derived from DSM-IV BPD criteria. The internal consistency of this checklist in a BPD population proved to be excellent (Cronbach alpha 0.93; Giesen-Bloo, Arntz, van Dyck, et al., 2001). 


\section{Results}

\section{Group comparability}

Fifteen patients (11 women, 4 men) with BPD, 14 patients ( 7 women, 7 men) with cluster C PD, 19 patients with axis I psychopathology (12 women, 7 men) and 21 non-patient controls (12 women, 7 men) participated in this study. BPD patients were significant younger than the other groups with a mean age of 30.9 years (MANOVA with mean group deviation contrasts, $p=0.008$ ). Mean age of the Cluster C PD group was 38.6 years, of the axis I psychopathology 39.2 years and of the controls 39.0 years. The groups did not differ significantly in terms of gender, educational level or proportion living with a partner. Age was the only variable for which further analyses were corrected.

\section{Janoff-Bulman's World Assumptive Model and Pretzer's Cognitive theory for BPD}

Mean scores on the WAS subscales paralleled to Pretzer's basic themes for BPD, the PDBQ-BPD subscale and test statistics of MANOVA deviation contrasts are summarized in Table 1. The overall multivariate between group effect was strongly significant, ( $\left.F_{\text {Hot }}(42,122)=3.68, p<0.001\right)$. As expected, BPD patients had significantly lower ratings on the subscales of benevolence of people and benevolence of the world compared to the other groups, confirming the hypothesis of BPD patients perceiving the world as less benevolent (or more malevolent) than non-patients. Non-patients had significantly higher benevolence ratings. The ratings on controllability, randomness and self-controllability, related to Pretzer's theme 'I am powerless and vulnerable', failed to show significant differences between the groups. However, on the subscale of luck BPD patients had the hypothesized significant lower scores than the other groups, indicating that BPD patients strongly perceive that they have less luck in their lives than other people. Non-patients rated significantly higher luck. The results for justice and self-worth, representing Pretzer's third theme 'I am unacceptable', were mixed. The average scores of the groups for justice weren't significantly different. With respect to self-worth, BPD patients did have a significant lower score than the other groups reflecting that BPD patients view themselves less positively than other people. Again, non-patients rated this scale significantly higher. The PDBQ-BPD subscale demonstrated its ability to discriminate between groups. The BPD patients made the highest belief ratings and differed significantly from the other groups. Axis I patients and non-patients made significantly lower belief ratings than the average rating. The belief ratings of Cluster $C$ patients were in between the BPD patients and the Axis I patients. 
Chapter 2

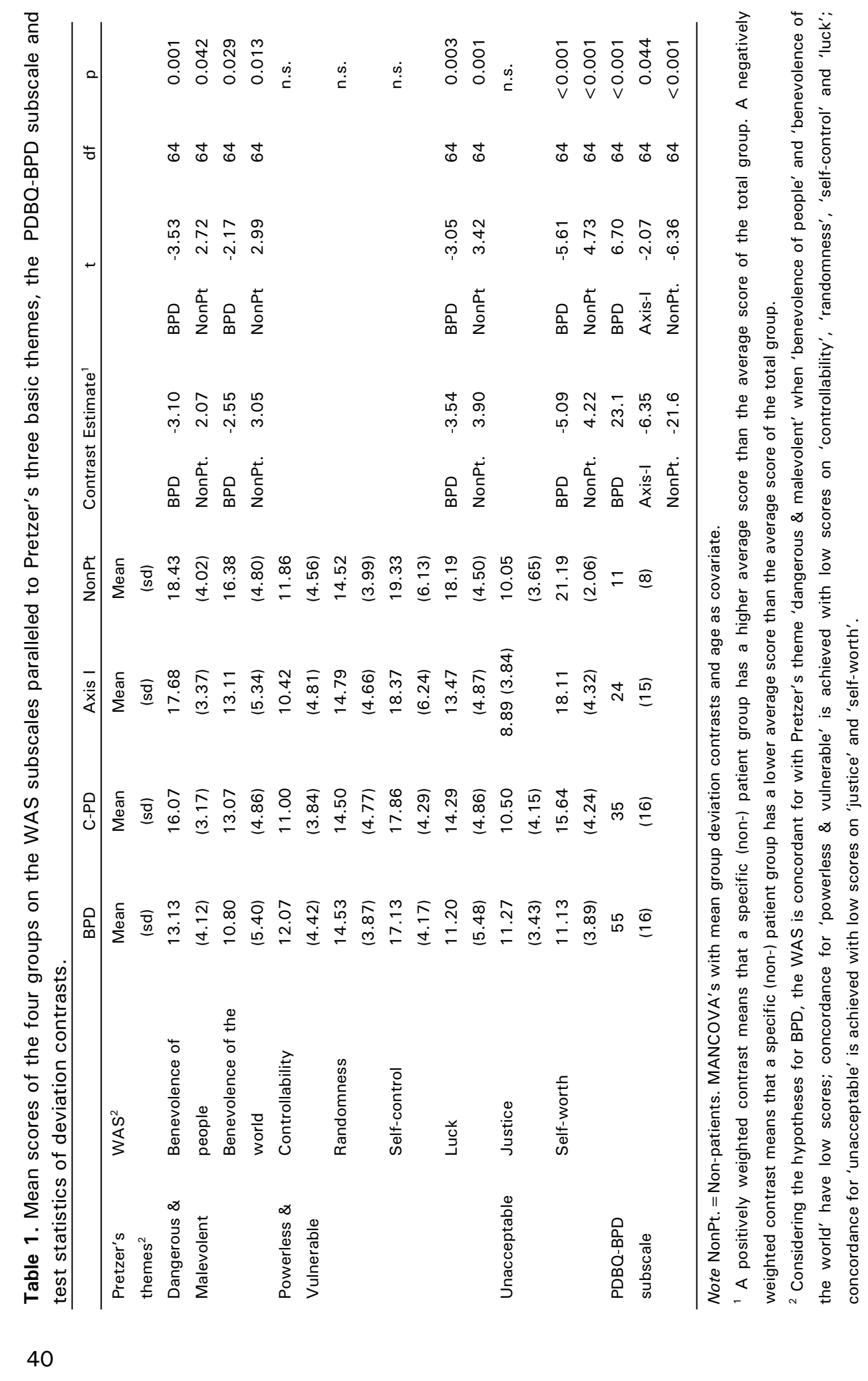




\section{The role of childhood trauma in world views}

The Pearson correlations of childhood trauma scores with those subscales of the WAS that were important in confirming Pretzer's theory for BPD, the PDBQ-BPD subscale and the BPD Checklist can be found in Table 2.

Table 2. Pearson correlations of trauma composite scores with those subscales of the WAS important in confirming Pretzer's theory for BPD, the PDBQ-BPD subscale and the BPD Checklist.

\begin{tabular}{llllllc}
\hline & WAS-BP & WAS-BW & WAS-SW & WAS-L & $\begin{array}{c}\text { PDBQ- } \\
\text { BPD }\end{array}$ & $\begin{array}{c}\text { BPD } \\
\text { Checklist }\end{array}$ \\
\hline Total trauma & $-0.29^{* *}$ & $-0.22^{*}$ & $-0.41^{* *}$ & $-0.26^{*}$ & $0.46^{* *}$ & $0.49^{* *}$ \\
Sexual abuse & $-0.28^{*}$ & $-0.23^{*}$ & $-0.34^{* *}$ & $-0.23^{*}$ & $0.37^{* *}$ & $0.38^{* *}$ \\
Physical abuse & $-0.24^{*}$ & -0.17 & $-0.24^{*}$ & -0.15 & $0.25^{*}$ & $0.31^{* *}$ \\
Emotional & & & & & & $0.46^{* *}$ \\
abuse/neglect & $-0.23^{*}$ & -0.16 & $-0.39^{* *}$ & $-0.27^{*}$ & $0.48^{*}$ \\
\hline
\end{tabular}

Note. ${ }^{*}=$ Significant at $p<0.05,1$-tailed

* ${ }^{*}$ Significant at $p<0.01,1$-tailed

The hypothesis that people who experienced a lot of childhood trauma perceive others and the world as less benevolent was confirmed. The relation between childhood trauma and self-worth showed, following the hypothesis, that someone with high trauma ratings sees him/herself less worthy than somebody else. The correlation between childhood trauma and luck indicates that people high on trauma consider themselves less lucky than other people. As expected, a strong positive relationship was found between childhood trauma and borderline specific beliefs. Another pronounced positive correlation was revealed between childhood trauma and borderline related psychopathology (BPD Checklist) at which more trauma was related to more borderline related psychopathology. Pearson correlations of the separate trauma composite scores: sexual abuse, physical abuse and emotional abuse/neglect with the specific WAS-subscales, PDBQ-BPD section and the BPD checklist, are mostly similar to the correlations of the total trauma composite score. However, physical and emotional trauma do not have a significant correlation with the assumptions of benevolence of the world by themselves. Physical abuse also fails to show a significant negative correlation with 'luck'.

The groups were compared on trauma before testing more detailed hypotheses on world views of BPD patients. An analysis of variance with age as covariate showed significant differences between the groups $(F(3,58)=12.90$, $p<0.001)$. BPD patients had the highest scores of experienced childhood trauma, gradually followed by Cluster C patients, Axis I patients and nonpatients. The cluster $\mathrm{C}$ patients formed the only group that, based on trauma 
scores, didn't stand out as a group amidst the other groups, as is shown by the deviation contrasts.

Table 3. Mean composite scores of the four groups on the structured trauma interview and test statistics.

\begin{tabular}{lccrrr}
\hline & $\begin{array}{l}\text { Trauma } \\
\mathrm{M}(\mathrm{sd})\end{array}$ & $\begin{array}{c}\text { Contrast Esti- } \\
\text { mate }\end{array}$ & $\mathrm{t}$ & $\mathrm{df}$ & $\mathrm{p}$ \\
\hline BPD $(n=15)$ & $93.73(30.72)$ & 40.29 & 5.54 & 58 & $<0.001$ \\
Cluster C $(n=14)$ & $56.21(39.27)$ & 0.57 & 0.08 & 58 & 0.813 \\
Axis I $(n=18)$ & $42.28(29.12)$ & -13.76 & -2.14 & 58 & 0.046 \\
Controls $(n=16)$ & $29.25(23.19)$ & -27.11 & -3.97 & 58 & $<0.001$ \\
\hline
\end{tabular}

Note. ANCOVA with mean deviation contrasts and age as covariate.

To examine if the content of world assumptions of BPD patients is explained by the high level childhood trauma these patients have experienced or by the presence of BPD psychopathology itself, a series of analyses of covariance where age and either trauma severity or BPD psychopathology severity were entered as covariates, were executed. Only the total trauma score will be used instead of the separate trauma scores to avoid loss of statistical power. The results of these analyses can be found in Table 4. It turned out that BPD patients view the world as less benevolent (more malevolent) and consider themselves less lucky compared to other people independent of the amount of childhood trauma. However, when corrected for severity of BPD psychopathology, the effects disappeared. Neither level of childhood trauma or BPD psychopathology affected the previous results of the assumptions of self-worth, for which BPD patients had the lowest ratings. The earlier results on the PDBQ-BPD section also weren't influenced by the use of trauma or borderline psychopathology severity as a covariate and remained solid.

Table 4. BPD patient's t-values and test statistics, contrasted to the three control groups, with age and childhood trauma or BPD related psychopathology as covariates of WAS relevant subscales paralleled to Pretzer's three basic themes and the PDBQ-BPD subscale.

\begin{tabular}{|c|c|c|c|c|c|c|c|c|c|}
\hline \multirow[t]{2}{*}{ Pretzer's themes } & \multirow[t]{2}{*}{ WAS } & \multicolumn{4}{|c|}{ Covariate Childhood Trauma } & \multicolumn{4}{|c|}{ Covariate BPD psychopathology } \\
\hline & & $\mathrm{CE}$ & $\mathrm{t}$ & $\mathrm{df}$ & $\mathrm{p}$ & $\mathrm{CE}$ & $\mathrm{t}$ & $\mathrm{df}$ & $\mathrm{p}$ \\
\hline Dangerous \& & $\mathrm{BP}$ & -3.08 & -2.62 & 57 & 0.011 & -1.69 & -1.57 & 57 & 0.122 \\
\hline Malevolent & BW & -2.89 & -1.86 & 57 & 0.068 & -0.81 & -0.55 & 57 & 0.584 \\
\hline Powerless \& & $\mathrm{L}$ & -3.51 & -2.48 & 57 & 0.016 & -1.41 & -1.02 & 57 & 0.313 \\
\hline \multicolumn{10}{|l|}{ Vulnerable } \\
\hline Unacceptable & SW & -5.28 & -4.74 & 57 & $<0.001$ & -3.37 & -3.12 & 57 & 0.003 \\
\hline \multicolumn{2}{|c|}{ PDBO-BPD subscale } & 24 & 5.48 & 57 & $<0.001$ & 12 & 3.46 & 57 & 0.001 \\
\hline
\end{tabular}

Note. $\mathrm{BP}=$ Perceived benevolence of people, $\mathrm{BW}=$ Perceived benevolence of the world, $\mathrm{L}=$ Luck, $\mathrm{SW}=$ Self Worth, $\mathrm{CE}=$ Contrast Estimate . 


\section{Discussion}

The present study had two goals: (1) testing whether Pretzer's basic themes for BPD are characteristic of BPD by using Janoff-Bulman's theoretical framework of world assumptions and (2) examining the role of trauma in the content of world views of BPD patients.

With respect to the first objective, using Janoff-Bulman's world assumptive model can largely support Pretzer's cognitive theory. BPD patients perceive the world and other people significantly less benevolent than other people or, fitting in Pretzer's first basic theme 'The world is dangerous and malevolent'. Pretzer's second theme 'I am powerless and vulnerable' was less supported by the results, only on the subscale of luck emerged a significant difference between the BPD patients and the other groups. BPD patients believe they are less lucky than other people or, in other words, they meet more adversity in their lives without having any logical arguments and chance is left as an explanation for these experiences. As for Pretzer's third theme 'I am inherently unacceptable', the most valuable subscale of the WAS in this respect, self-worth, proved that BPD patients tend to view themselves significantly less positive than other people. The subscales of controllability, randomness/chance and self-controllability (related to Pretzer's second theme) and justice (related to Pretzer's third theme) didn't give significant results. However, when taking a closer look at the phrasing of the items this is not much of a surprise. The items inquire into the efforts and actions a person has undertaken instead of inquiring in how these efforts and actions effectively work out for the person him/herself. A second issue for controllability, randomness and justice is that the items about the world are formulated in a very general manner, they are not about the position of the person him/herself in the world or related to other people, but about people in general. This non-personal formulation might have undermined the validity of these items.

The results related to the second aim of the study demonstrated, as hypothesized, mostly significant negative correlations between experienced childhood trauma and those WAS-subscales supporting Pretzer's cognitive theory for BPD. Separate physical abuse and emotional abuse/neglect scores did not show significant correlations with 'benevolence of the world', which was possibly caused by the relatively small sample size. The limited internal consistency of the 'luck' subscale of the WAS offers a possible explanation for the non-significant relation between physical abuse and this subscale. The data confirmed the expectation that BPD patients experienced significantly more childhood trauma than the other groups. As for the content of world assumptions of BPD patients in this study, it can be concluded that these can better be explained by the presence of BPD than by the presence and severity of childhood trauma. This sug- 
gests that not childhood trauma in itself, but only the way it is processed and becomes part of personality, determines basic world views.

The finding that people who experienced more trauma hold more negative world assumptions with respect to benevolence of the world, perceived selfworth and luck is largely consistent with Janoff Bulman's results (1989). The current study showed a more distinct effect of trauma on the category of benevolence of the world than in Janoff Bulman's study, where a trauma effect was found in the assumptions about the impersonal world but not in the assumptions about other people. Perhaps the use of different groups of participants in this study, patients of mental health care institutions versus undergraduate students, accounts for this contrast.

A second aspect is that Janoff-Bulman (1989) demonstrated a negative influence of trauma on the distributional principle of chance whereas this study demonstrated a negative influence of trauma on the self-relevant dimension of chance, luck. However, this can be understood by looking into the definition of trauma in both studies and their relation to chance and luck. For Janoff-Bulman (1989), trauma was defined as the occurrence of one (or more) extreme negative event during a person's live, namely: death of a parent, death of a sibling, incest, rape, fire that destroyed their home and an accident that resulted in their own serious disability. In this study, trauma involved many different types of physical, sexual and emotional abuse or neglect in childhood and was calculated to a weighed composite score. Even though one can see certain overlap between these two sets of trauma, one can argue on a rational basis that most trauma of the first study, if occurring, happen primarily without any (possible) personal involvement of a respondent and are often caused through external factors (e.g. parent dies of illness, house burnt down because of a short circuit, disability in car crash while in the passenger seat) and can therefore be linked with the assumptions of chance, that are formulated in a general, non-personal way. Traumata in the present study implicitly comprehend individual involvement/interaction in childhood (forced sexual actions, 'disciplinary' beatings). So, the interpersonal aspect(s) of traumata are pivotal in the used trauma-interview, an explicit contrast with Janoff-Bulman's study where traumata were mostly not interpersonal. This observation leaves a more prominent role for individual information processing which, in turn, leads to internal attributions and can therefore be linked with the self-relevant assumptions of luck. Based on this argumentation the found trauma-effect on luck instead of chance seems a natural consequence.

Considering that the traumatized BPD patients were on average considerably older than the traumatized undergraduate students $(30.9$ years versus $<25$ years) whereas both studies mainly focused on the effect of childhood trauma, it can be noted that world assumptions appear stable over an even longer period 
than Janoff-Bulman already found. Yet, it is not unquestionable clear if this stability of world views is really due to the presence of experienced childhood trauma since the analyses of the current data have shown that, once corrected for BPD psychopathology severity, world views of BPD patients do not differ from other patient and non patient groups while WAS self-worth and BPD specific beliefs keep their discriminating ability. In a prospective study, Gluhoski \& Wortman (1996) assessed the influence of trauma on changes in world views and found negative effects on self-view and vulnerability. The effect on self-view is comparable to the effect on self worth in the current study. The increased vulnerability views after experiencing trauma can be paralleled, considering the previous remarks on personal involvement in trauma, to the results on chance (Janoff-Bulman, 1989) and luck (present study). However, the reliability and validity of Gluhoski and Wortman's trauma instrument was not assessed and the operalization of their world view constructs can be discussed. Another aspect is that the prospective part of their study made that the subjects experienced trauma mainly during their adult life (average age 53 years). The difference between the types of trauma (e.g. divorce, change of residence and serious financial difficulties) of Gluhoski and Wortman and our study further complicates a generalization of their findings to the area of BPD.

The specificity of BPD beliefs and the observed pattern of beliefs over the different groups in the present study are in line with previous findings (Arntz, Dietzel, et al., 1999; Arntz, Dreessen, et al., 2004) This confirms the association of personality disorder related beliefs with (personality) psychopathology in general but, like before, does not prove the theory that PD's are caused by trauma. Nevertheless, the present results further suggest that the PDBQ-BPD subscale is a more specific measure of BPD pathology than the WAS subscales.

Not all hypotheses of this study could be accepted. One probable explanation is the relative small sample size. A larger sample size would probably increase the robustness of the current findings, maybe even confirm other proposed hypotheses, and enlarge the external validity. A suggestion for future research would be to investigate world views in different trauma groups, for example a specific group of patients with post traumatic stress disorder (without PD's) next to general Axis I patients. The differences in impact of trauma experience during childhood or during adult life on world assumptions also deserve attention.

In sum, it can be concluded that Pretzer's theory for BPD (Pretzer, 1990) can be largely supported through Janoff-Bulman's world assumptive model (Janoff-Bulman, 1989). Furthermore, world assumptions of BPD patients can better be explained by the severity of BPD psychopathology than by the presence of trauma. 



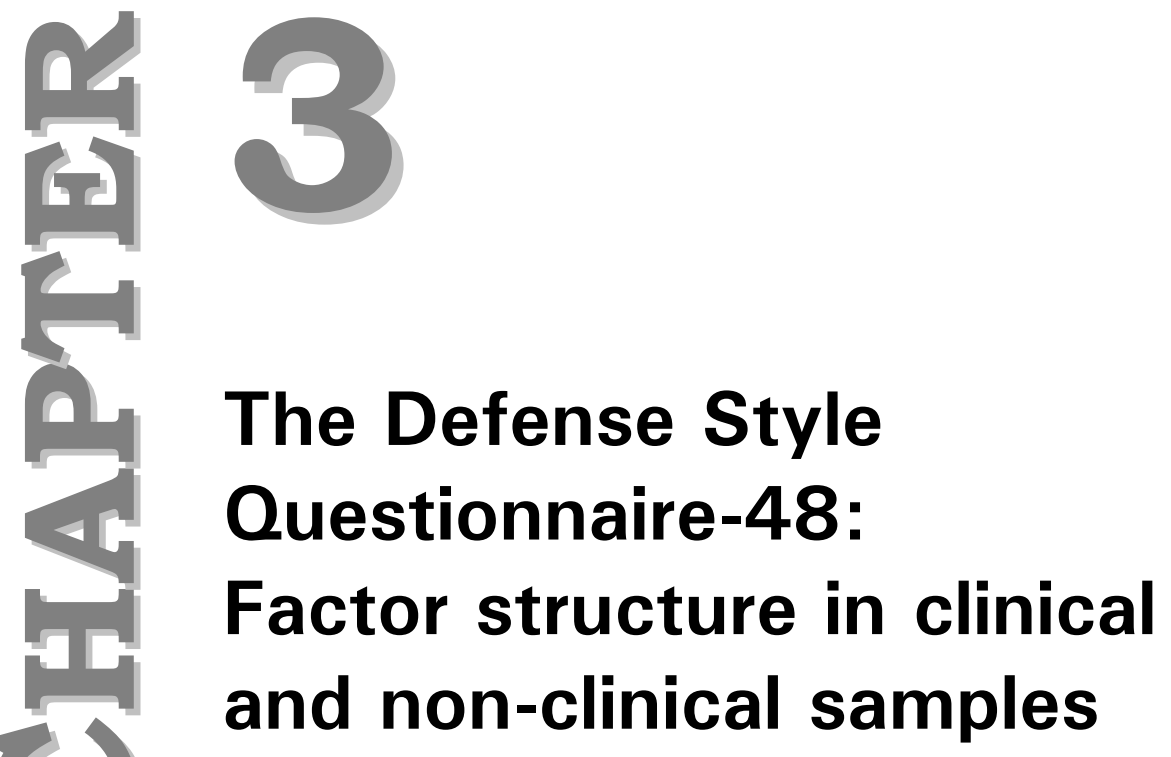

Giesen-Bloo, J., Arntz, A., Opdenacker, J., Spinhoven, P. (Submitted for publication) 


\title{
Chapter 3
}

\begin{abstract}
Focus of the present study is the 48-item Defense Style Questionnaire (DSQ-48). Central aims are to replicate the three-factor structure of the DSQ-36 (Spinhoven, van Gaalen, et al., 1995), to assess the psychometric properties and to compare the defensive style of different patient and non-patient groups. 129 BPD patients, 55 Cluster C personality disorder patients, 45 patients with only Axis-I psychopathology and 53 non-patients participated in the study. Validation data were obtained with self-report questionnaires and a semi-structured interview. The findings of Spinhoven, van Gaalen, et al. (1995) were replicated alas with identical psychometric shortcomings. Subsequent explorative principal component analysis revealed a five-factor structure (distrust, self-protection, self-sacrifice, avoidance and control) with better psychometric features. Replication in a new sample of 472 non-patients demonstrated satisfactory psychometric properties of this five-factor structure.
\end{abstract}




\section{Introduction}

The concept of defense mechanisms has been used, especially in the psychoanalytic literature, for a long time but not in a clear and consistent way. Defense mechanisms are used to explain certain intrapsychic processes which are believed to be unconscious (Bond, Gardner, Christian, et al., 1983). Classical psychoanalysts viewed the use of defense mechanisms compensatory to the expressions of drives but according to modern psychoanalysts (self-psychology, object-relation theory) defense mechanisms also serve to regulate the effects of stress and to maintain and protect self-esteem and individual personality organization (Cooper, 1998).

The unconscious aspect of defense mechanisms troubled their reliable and valid assessment by clinical interviews, projective methods or vignettes for a long time. Other developed (scoring) methods (e.g. Cramer, 1999; Cramer \& Blatt, 1990; Davidson \& MacGregor, 1996; Lerner, Albert, \& Walsh, 1987; Perry $\&$ lanni, 1998) improved the reliability and validity of the assessment of defense mechanisms but more practical disadvantages as high costs, need of experienced and trained raters, and highly time-consuming procedures did not change. Bond, Gardner, et al. (1983) argued that, with the premises that persons can become aware of the defenses they use at times when defenses fail temporarily or through comments of other people, it must be possible to develop a selfreport questionnaire for defense mechanisms. This premise is also based on the belief that people are able to comment on their behavior, unaware of its defensive function. The result was the Defense Style Questionnaire (DSO; Bond, Gardner, et al., 1983) consisting of 81 items that represent various defense mechanisms, which must be rated on a 9-point Likert scale from 'strongly disagree' to 'strongly agree'. Further development, for both practical and theoretical reasons, led to a 36-item DSO (Andrews, Pollock, \& Stewart, 1989; Andrews, Singh, \& Bond, 1993). The results of a study of this short form DSO (Muris \& Merckelbach, 1994) were supportive of construct validity and test-retest reliability. Spinhoven, van Gaalen, et al. (1995) examined the psychometric properties and factorial structure of the Dutch version of the short 36-item DSO. The found three-factor structure (immature, neurotic and mature defenses) of the 36-item DSO was very similar to the structure found by Andrews, Pollock, et al. (1989), as was construct validity. However, the results suggested limited discriminant validity between different groups of psychiatric patients since group differences disappeared after controlling for neuroticism symptoms. Moreover, the results indicated that the distribution of the 36-items clearly deviated from a normal distribution, that the amount of variance explained with principal component analysis (PCA) was relatively small and that the reliability of the factors was insufficient. The neuroticism factor in particular was weak and consisted of a too small 
number of items (4). A strong neurotic factor is desirable to let the DSO adequately serve as a (treatment) outcome measure, as well as for clinical and research purposes. We therefore modified the 36-item DSO because of these shortcomings; several items were rephrased, and 1 mature item and 11 neurotic items of Bond's 88-item DSQ (1983) were added. The current DSQ-version therefore consists of 48 items, representing 18 defense mechanisms.

Most studies on defensive functioning are limited to patients with DSM Axis-I psychopathology and/or non-patients without evaluation of axis II diagnostics and psychopathology (e.g. Andrews, Pollock, et al., 1989; Andrews, Singh, et al., 1993; Pollock \& Andrews, 1989; Sinha \& Watson, 1999; Spinhoven, van Gaalen, et al., 1995). Theoretical and clinical arguments, of which the view that defensive style is part of one's personality structure is probably the most obvious (e.g. Kernberg, 1996), promote an extended focus to investigate the relations between DSM Axis-II personality disorders and defensive functioning. Several studies which did include patients with personality disorders (PD) besides patients with no personality pathology and non-patients demonstrated that PDpatients used more immature defenses than axis-I patients and non-patients (Bond, Paris, \& Zweig-Frank, 1994; Devens \& Erickson, 1998; Paris, ZweigFrank, Bond, et al., 1996; Sammallahti \& Aalberg, 1995; Sammallahti, Aalberg, $\&$ Pentinsaari, 1994). The relation of immature defensive style and personality disorders was found to be most apparent in patients with a borderline personality disorder (BPD; Bond, Paris, et al., 1994; Devens \& Erickson, 1998; Lerner, Albert, et al., 1987).

The aim of the present study on the DSQ-48 was threefold: (1) to replicate the three-factor structure with immature, neurotic and mature defenses of the DSQ36 , (2) to assess the psychometric properties (homogeneity, internal consistency, discriminant validity, concurrent and construct validity) and (3) to examine and compare the defensive style of BPD-patients, Cluster-C PD patients, patients with only Axis-I psychopathology and non-patients. With respect to validity, it was hypothesized that immature and neurotic defense style would correlate significantly positive with general psychopathological complaints (SCL-90; Arrindell \& Ettema, 1986; Derogatis, Lipman, \& Covi, 1973), with self-ideal discrepancy (Miskimins, Wilson, Braucht, et al., 1971), with specific BPD beliefs (PDBQ-BPD section; Arntz, Dietzel, et al., 1999; Arntz, Dreessen, et al., 2004), with maladaptive schemas (YSQ; Rijkeboer, van den Bergh, \& van den Bout, 2005; Schmidt, Joiner, Young, et al., 1995), and with pathological borderline personality organization features as formulated by Kernberg (IPO; Arntz \& Kerkhofs, 1999; Kernberg \& Clarkin, 1995; Lenzenweger, Clarkin, Kernberg, et al., 2001). Immature defense style should also have a significant positive relation with observed frequency and severity of BPD symptoms (BPDSI-IV; Arntz, van den Hoorn, Cornelis, et al., 2003; Giesen-Bloo, Wachters, Schouten, et al., 2006) and with experienced burden of BPD symp- 
toms (BPD-Checklist; Giesen-Bloo, Arntz, \& Schouten, 2006), whereas neurotic defense style should have a null correlation with these measures. A significantly negative relation was expected between immature and neurotic defense style and self-esteem (Rosenberg, 1965). Mature defense style was thought to show significant relations with validation measures that are opposite to those of immature defense style. Statistical procedures of Spinhoven, van Gaalen, et al. (1995) were followed as much as possible considering the current data set characteristics. Furthermore, it was hypothesized that BPD-patients would demonstrate significantly larger use of immature and neurotic defenses than the other groups. Cluster-C PD patients should have a significantly larger use of immature and neurotic defenses than Axis-I patients and non-patients, as Axis-I patients would use significantly more immature and neurotic defenses than non-patients. Nonpatients were expected to have a significantly larger use of mature defenses than all patient groups.

\section{Method}

\section{Participants}

129 BPD-patients, 55 Cluster-C PD patients and 45 patients with only AxisI psychopathology were concurrently recruited from twelve mental health institutions (inpatient and outpatient) for participation in a series of studies including the present one. Participation to the study was voluntary and could be stopped at any time. 53 Non-patients were recruited through advertisements in local newspapers. Non-patients received gift certificates, rated to the amount of time they participated in the series of studies, with an average worth of 30 euro. After a complete description of the study to all subjects, written informed consent was obtained. Diagnoses were assessed with DSM-IV based semi-structured interviews (SCID-I, SCID-II; First, Gibbon, et al., 1997; First, Spitzer, et al., 1996; Groenestijn, Akkerhuis, et al., 1999; Weertman, Arntz, et al., 2000). All subjects had to be between age 18 and 60. General exclusion criteria were psychotic disorders, bipolar disorder and mental retardation. BPD-patients were allowed to have comorbid personality and/or Axis-I disorders. Cluster-C patients had to meet criteria for at least one Cluster-C PD, were allowed to have comorbid Axis-I disorders but were not allowed to have an additional cluster-A or -B personality diagnosis or more than 2 BPD criteria. Patients with Axis-I psychopathology had to meet criteria for at least one Axis-I disorder, were not allowed to have threshold diagnoses of PD's or meet more than 2 BPD criteria. Non-patients had to be free of psychological complaints, could not have any Axis-I disorder, PD, PD threshold diagnosis or meet more than 2 BPD criteria. 


\section{Instruments}

After completing the diagnostic SCID-interviews in 1 or 2 sessions, the 48item DSO and the following psychometrically robust instruments were administered in another session:

The borderline personality disorder severity index -IV (BPDSI-IV; Arntz, van den Hoorn, et al., 2003; Giesen-Bloo, Wachters, et al., 2006), is a DSM-IV based semi-structured interview, designed to assess the frequency and severity of manifestations, specific for BPD, during the past three months.

The BPD checklist (Giesen-Bloo, Arntz, et al., 2006) is designed to assess the burden of borderline related symptoms during the last month. Subjects indicated on a 5-point Likert scale from 'not at all' to 'extremely' how much they were troubled by 47 different complaints, derived from DSM-IV BPD criteria.

The Personality Disorder Beliefs Questionnaire - BPD section (PDBQ; Arntz, Dietzel, et al., 1999; Arntz, Dreessen, et al., 2004) is developed after Beck's theory of PD (Arntz, 1994b; Beck, Freeman, et al., 1990). It includes 20 beliefs, specific for BPD. The strength of each belief is rated on $100 \mathrm{~mm}$ visual analogue scales with "I don't believe this at all" on one end and "I believe this completely" on the other end.

The Rosenberg Self Esteem Scale (RSES; Rosenberg, 1965) consists of 15 items. Subjects indicate on a 6-point Likert scale from "completely true" to "not true at all" how much they are in agreement with each statement.

Miskimins' self-goal-other discrepancy scale (Miskimins, Wilson, et al., 1971) was used to assess mean self-ideal and self-other discrepancies. 15 pairs of personality characteristics have to be rated on $100 \mathrm{~mm}$ visual analogue scales ('happy' on one end, 'unhappy' on the other end) from different points of view: how am I? (self), how do other people perceive me? (other) and how do I want to be? (ideal).

The Symptom Checklist -90 (SCL-90; Arrindell \& Ettema, 1986; Derogatis, Lipman, et al., 1973) assesses general physical and psychological complaints and gives a general measure of psychopathology. Subjects rate to what extent they were bothered by 90 listed complaints during the last week on a 5-point Likert scale from "not at all" to "very much".

The items of the self-report Inventory of Personality Organization (IPO; Arntz \& Kerkhofs, 1999; Kernberg \& Clarkin, 1995; Lenzenweger, Clarkin, et al., 2001) reflect a borderline personality organizational structure. The characterizing 90 items concern identity diffusion, use of lower level defenses, alterations in reality testing, pathological object relations (narcissistic and poor quality) and superego pathology, rated on a 5-point Likert scale from "never true" to "always true". This study only focused on the sum score of someone's use of lower level defenses (14 items). 


\section{Data analysis}

The statistical procedures of Spinhoven, van Gaalen, et al. (1995) were largely repeated in the total group and in the separate groups. After testing the distribution of items, confirmatory factor analysis with simultaneous components analysis was executed (SCA; Kiers, 1990; Nunnally, 1978). SCA works with an a priori matrix in binary form where each variable receives a weight of 1 on the component to which it supposedly belongs and zeroes for the other components. This multiple group method is especially suitable for designs where (identical) factors are to be defined in more than two groups (Millsap \& Meredith, 1988). To check if the simultaneous components adequately summarize the information in the populations, the amount of variance explained with SCA will be compared to that of a principal component analysis (PCA) on the same information. Homogeneity and internal consistency of the factor scales were determined by calculating mean inter-item correlations, item-rest correlations and Cronbach's alpha's for each factor. Items had to load at least 0.40 on their factor with a difference in loadings on other factors of at least 0.20. Mean inter-item correlations were expected to range between 0.20 and 0.40 , item-rest correlations were expected to be above 0.30. Correlations between factors were supposed be less than 0.60 (Briggs \& Cheek, 1986). Internal consistencies were satisfactory if Cronbach's $\alpha$ was above 0.70 (Nunnally, 1978). Construct and concurrent validity were examined with Pearson product moment correlations and point biserial correlations. Discriminant validity was to be determined with MANCOVA's and, if indicated, univariate analyses followed by post hoc Scheffe contrasts.

\section{Results}

\section{Group comparability}

Demographic data on the four groups are summarized in Table 1. Seventytwo percent of the Axis-I patients had an anxiety disorder as primary diagnosis, 14 percent a mood disorder, 7 percent an eating disorder, 5 percent a disorder due to a general medical condition and 2 percent a somatoform disorder.

BPD-patients were on average significantly younger than all other groups and had on average significantly more Axis-I diagnoses than Cluster C or Axis-I patients. Compared to Axis-I patients and non-patients significantly more BPDpatients were not employed, but on disability or welfare. Therefore, further between-group analyses (discriminant validity) were corrected for these variables. 
Table 1. Demographic data and number of Axis-I diagnoses on all groups

\begin{tabular}{lccccccccc}
\hline & \multicolumn{2}{c}{$\begin{array}{c}\text { BPD } \\
\text { patients } \\
\end{array}$} & $\mathrm{N}=129)$ & \multicolumn{2}{c}{$\begin{array}{c}\text { Cluster-C } \\
\text { patients } \\
(\mathrm{N}=55)\end{array}$} & $\begin{array}{c}\text { Axis-I } \\
\text { patients } \\
(\mathrm{N}=45)\end{array}$ & $\begin{array}{c}\text { Non-clinical } \\
\text { controls } \\
(\mathrm{N}=53)\end{array}$ \\
\hline Women & 115 & 90 & 35 & 64 & 32 & 71 & 33 & 62 \\
\hline & Mean & SD & Mean & SD & Mean & SD & Mean & SD \\
\hline Age & $30.04^{*}$ & 7.42 & 37.33 & 9.54 & 33.64 & 10.54 & 36.55 & 15.02 \\
Educational level & 7.48 & 2.25 & 7.89 & 2.29 & 7.82 & 1.87 & 7.81 & 2.18 \\
Employment status & $4.70^{* *}$ & 2.85 & 4.20 & 2.36 & 3.82 & 2.06 & 3.60 & 2.45 \\
Number of & $2.42^{* *}$ & 1.47 & 1.80 & 1.22 & 1.49 & .86 & - & - \\
Axis-I disorders & & & & & & & & \\
\hline$*$ & $P<.05$ for BPD patients vs Cluster C patients, vs Axis-I patients and vs non-patients. \\
$* *$ & $P<.05$ for BPD patients vs Axis-I patients and vs non-patients. & & \\
$* * *$ & $P<.01$ for BPD patients vs Cluster C patients and vs Axis-l patients. & &
\end{tabular}

Factor structure, homogeneity and internal consistency

The Kolmogorov-Smirnov one-sample test revealed that all 48 items scores were not normally distributed in either the total sample or the separate BPDgroup. Thirty-four of the DSO-items in the Cluster C PD-group were not normally distributed, 24 in the Axis-I group and 36 in the non-patient group. A normal distribution is not an imperative assumption for executing factor analyses but a consequence of these skewed distributions is that one can expect smaller amounts of explained variance than under optimal conditions. SCA revealed that the immature, neurotic and mature factor structure explained $27.3 \%$ of the total variance in the total group, with a loss of $3,4 \%$, compared to the accompanying PCA. This finding is similar to the results of Spinhoven, van Gaalen, et al. (1995) and Andrews, Pollock, et al. (1989). In the different patient groups SCA explained 20.9 to $25.5 \%$ of the total variance with the current 3 -factor model (losses with PCA between 3.2 to $7.6 \%$ ). However, in the non-patient group, the model explained $30.9 \%$ of the total variance ( $4.5 \%$ less than PCA).

Table 2 summarizes the factor scales' homogeneity indicators and internal consistency measure in the total group and the separate groups. 
Table 2. Mean inter-item correlations, range item-remainder correlations, and Cronbach's alpha for each group and the total group

\begin{tabular}{|c|c|c|c|c|}
\hline BPD patients ( $n=129)$ & $\begin{array}{c}\text { Mean } \\
\text { item loading } \\
\text { (range) }\end{array}$ & Mean rii & $\begin{array}{l}\text { Mean rir } \\
\text { (range) }\end{array}$ & Alpha \\
\hline Factor 1 Immature defenses & $.44(.08 .75)$ & .16 & $.36(-.02 .69)$ & .80 \\
\hline Factor 2 Neurotic defenses & $.45(.26 .57)$ & .13 & $.31(.13 .46)$ & .70 \\
\hline Factor 3 Mature defenses & $.45(.15 .51)$ & .10 & $.23(.02 .39)$ & .55 \\
\hline \multicolumn{5}{|l|}{ Cluster C PD patients ( $n=55)$} \\
\hline Factor 1 Immature defenses & $.40(-.01 .66)$ & .12 & $.30(-.14 .57)$ & .75 \\
\hline Factor 2 Neurotic defenses & $.40(-.03 .72)$ & .06 & $.19(-.04 .61)$ & .53 \\
\hline Factor 3 Mature defenses & $.41(.07 .56)$ & .08 & $.19(-.14 .36)$ & .47 \\
\hline \multicolumn{5}{|l|}{ Axis-I patients $(n=45)$} \\
\hline Factor $1 \mathrm{Immature}$ defenses & $.45(-.00 .67)$ & .16 & $.35(-.11 .65)$ & .79 \\
\hline Factor 2 Neurotic defenses & $.33(.18 .74)$ & .08 & $.21(.01 .65)$ & .58 \\
\hline Factor 3 Mature defenses & $.42(.01 .69)$ & .08 & $.21(-.21 .53)$ & .48 \\
\hline \multicolumn{5}{|l|}{ Non-patients $(n=53)$} \\
\hline Factor 1 Immature defenses & $.52(.24 .75)$ & .23 & $.44(.16 .68)$ & .84 \\
\hline Factor 2 Neurotic defenses & $.46(.29 .60)$ & .16 & $.34(.16 .49)$ & .74 \\
\hline Factor 3 Mature defenses & $.49(.32 .57)$ & .15 & $.30(.12 .40)$ & .63 \\
\hline \multicolumn{5}{|l|}{ Total group ( $n=282)$} \\
\hline Factor $1 \mathrm{Immature}$ defenses & $.52(-.08 .75)$ & .23 & $.45(-.18 .70)$ & .87 \\
\hline Factor 2 Neurotic defenses & $.43(.19 .61)$ & .13 & $.30(.04 .51)$ & .71 \\
\hline Factor 3 Mature defenses & $.43(.15 .59)$ & .09 & $.22(-.08 .41)$ & .52 \\
\hline
\end{tabular}

Note. $r_{\text {ii }}=$ inter-item correlation; $r_{\text {ir }}=$ item-rest correlation.

Homogeneity was not very satisfactory since only the mean inter-item correlations of the immature defense factors of the total group and the non-patients lie in the optimal range of .20-.40. Most items showed significantly positive correlations with other factor items. Three items (suppression (41), idealization (47) and denial (18)) held significantly negative correlations with some of their other factor items. The internal consistency of the immature factor ranged from satisfactory to good. Cronbach's alpha of the neurotic factor was insufficient for the Cluster C PD group and the Axis-I psychopathology group but satisfactory for the total, BPD and non-clinical groups. The mature factor lacked internal consistency in all groups.

Pearson correlations between DSQ factor scores and the demographic variables in the total group were all but one, below 0.20 (Surwillo, 1980). Age had a significant negative correlation with immature defenses $(r=-.20, P=.001)$. Results in each group separately were highly similar. Exceptions were significantly negative correlations between gender and immature defenses in the Axis-I group $(r=-.33, P=.027)$ and in the non-clinical group $(r=-.28, P=.044)$, and a signifi- 
cantly positive correlation between educational level and mature defenses in the non-clinical group $(r=.43, P=.002)$.

\section{Concurrent and Construct validity}

Pearson correlations between defense factors, and with the other instruments, for the total group and test statistics are depicted in Table 3.

Table 3. Pearson product moment correlations between defensive factors, with other instruments and test statistics for the total group.

\begin{tabular}{|c|c|c|c|c|}
\hline & & \multicolumn{3}{|l|}{ DSQ factors } \\
\hline & & $\begin{array}{l}\text { 1. Immature de- } \\
\text { fenses }\end{array}$ & $\begin{array}{l}\text { 2. Neurotic De- } \\
\text { fenses }\end{array}$ & 3. Mature defenses \\
\hline Factor 1 & $(n=282)$ & - & $.54 * *$ & .05 \\
\hline Factor 2 & $(n=282)$ & .68 & - & $.13^{*}$ \\
\hline Factor 3 & $(n=282)$ & .07 &.$\overline{21}$ & _- \\
\hline SCL-90 & $(n=281)$ & $.65 * *$ & $.47 * *$ & $-.13 *$ \\
\hline BPDSI-IV & $(n=239)$ & $.61 * *$ & $.34 * *$ & $-.15^{*}$ \\
\hline BPD Checklist & $(n=247)$ & $.70 * *$ & $.45 * *$ & $-.16 *$ \\
\hline Self-Ideal Discrepancy & $(n=226)$ & $.65 * *$ & $.40 * *$ & $-.25^{* *}$ \\
\hline RSES & $(n=245)$ & $-.39 * *$ & $-.26 * *$ & $.22 * *$ \\
\hline PDBQ-BPD section & $(n=278)$ & $.74 * *$ & $.56 * *$ & -.10 \\
\hline IPO-lower level & $(n=108)$ & $.75 * *$ & $.55 * *$ & .06 \\
\hline
\end{tabular}

Note. With respect to factor correlations: above diagonal pearson $r$, below diagonal pearson $r$ corrected for attenuation. ${ }^{*} P<.05,{ }^{*} P<.001$. SCL-90: Symptom Checklist-90; BPDSI-IV: Borderline Personality Disorder Severity Index-IV; RSES: Rosenberg Self Esteem Scale; PDBQ: Personality Disorder Belief Questionnaire; IPO-lower level: Inventory of Personality Organization use of lower level defenses.

Immature defenses showed a large and significantly positive correlation with neurotic defenses. Immature defenses and mature defenses appeared to have no relation at all. The correlation of neurotic defenses with mature defenses was small and significantly positive. All measures held the hypothesized relative correlations with the DSQ-factor scores in the total group (although two correlations of mature defenses with the PDBQ-BPD section and with the IPO-lower level defenses did not reach a significant level), supporting an order of immature, neurotic and mature defenses.

\section{Discriminant validity}

Table 4 contains mean DSQ-subscale scores with standard deviations and test statistics for the total group and for all separate groups. 
Table 4. Factor scores for each group for the DSQ-48.

\begin{tabular}{lccccccccc}
\hline & \multicolumn{2}{c}{$\begin{array}{c}\mathrm{BPD} \\
\text { patients } \\
(\mathrm{N}=129)\end{array}$} & \multicolumn{2}{c}{$\begin{array}{c}\text { Cluster-C } \\
\text { patients } \\
(\mathrm{N}=55)\end{array}$} & \multicolumn{2}{c}{$\begin{array}{c}\text { Axis-I } \\
\text { patients } \\
(\mathrm{N}=45)\end{array}$} & $\begin{array}{c}\text { Non-clinical } \\
\text { controls } \\
(\mathrm{N}=53)\end{array}$ \\
\hline Factor scale scores & Mean & $S D$ & Mean & $S D$ & Mean & $S D$ & Mean & $S D$ \\
\hline 1. Immature defenses & $4.27^{\mathrm{a}}$ & 1.10 & $3.36^{\mathrm{b}}$ & 0.93 & $2.70^{\mathrm{c}}$ & 0.90 & $2.37^{\mathrm{c}}$ & 0.91 \\
2. Neurotic defenses & $4.95^{\mathrm{a}}$ & 1.09 & $4.46^{\mathrm{b}}$ & 0.83 & $4.14^{\mathrm{b}, \mathrm{c}}$ & 0.93 & $3.82^{\mathrm{c}}$ & 1.03 \\
3. Mature defenses & $4.85^{\mathrm{a}, \mathrm{b}}$ & 1.09 & $4.61^{\mathrm{a}}$ & 0.99 & $4.90^{\mathrm{a}, \mathrm{b}}$ & 1.03 & $5.26^{\mathrm{b}}$ & 1.13 \\
\hline
\end{tabular}

Note. Groups with a different suffix are significantly different from each other with at least $P<.05$.

DSQ-subscale score distributions were normally distributed. A multivariate analysis with group as independent variable, the DSQ-factor scores as dependent variables, and age and employment status as covariates revealed a significant main effect for group $(F(9,834)=15.86, P<.001)$. Contributions of the covariates were non significant. Subsequent univariate analyses of variance with Scheffe contrasts for each DSQ-factor demonstrated that BPD patients have a significantly higher use of immature and neurotic defenses than both patient and non-patient groups. $\mathrm{Pa}$ tients with Cluster C PD's had significantly higher ratings on immature defenses than Axis-I patients and non-patients, and significantly higher ratings on neurotic defenses than non-patients. No significant differences were observed between Axis-I patients and non-patients.

\section{Post hoc analyses}

Recapitulating the planned data analyses results, we replicated the findings of Spinhoven, van Gaalen, et al. (1995) and Andrews, Pollock, et al. (1989) with respect to the immature, neurotic and mature defense factor structure. However, factorial homogeneity, internal consistency and discriminant validity were limited, whereas concurrent and construct validity were satisfying.

Results of a SCA analysis on the DSQ-36 items of Andrews and Spinhoven, as a subset from the present DSQ-48, did replicate the 1989 and 1995 findings very well, alas with identical shortcomings (data not shown). So, the expected psychometric improvements of the DSQ-48 compared to the DSQ-36, were not found. It became therefore interesting to examine whether it was possible to find a different factor structure with better psychometric features. Fourteen of the eigenvalues accompanying the initial SCA of the DSQ-48 for the total group were above 1. A hypothesis following this observation is the possible absence of the higher order factor model, which the hypothesized 3-factor model was, and the presence of a multi-factorial model like one representing the 18 different defense mechanisms. Based on SCA, the 18 defense mechanisms structure was responsible for $60,6 \%$ of the total explained variance in the total group com- 
pared to $70,0 \%$ explained variance with an 18-factor PCA. Homogeneity of the 18 defense mechanism factors was insufficient. The probability of a satisfactory multi-factorial solution for the current defense mechanism definitions (Bond, Gardner, et al., 1983) was then dismissed and further statistic evaluation of the DSQ-48 was initiated out of item level.

Figure 1. Scree plot of the DSQ-48

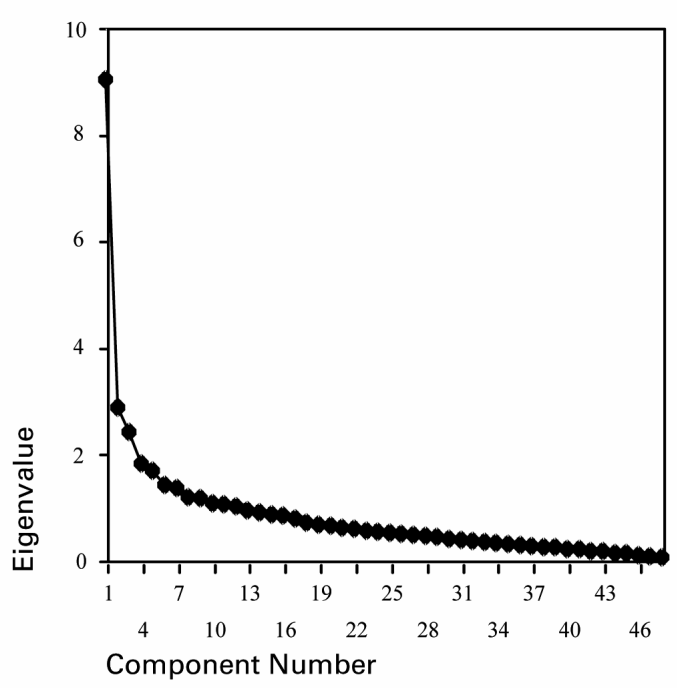

Procedure Based on the scree plot (Figure 1; Cattell, 1966), 3-, 4- and 5-factor solutions were explored for the total study group.

First, principal component analyses (PCA) with varimax rotation and followed by oblique rotation, with either 3,4 or 5 factors, were executed. To assign an item to a factor, the item loading had to be larger than 0.40 and differ at least 0.20 with each other. The assigned items were then interpreted for their content which led to more specific and defining factor labels. Subsequently were the yet unassigned items reviewed and assigned to a factor when both the item loading was larger than 0.20 and the item content fitted the factor definition. Further items that could not be assigned, were left out. The 3-, 4- and 5-factor model that appeared, were then subjected to SCA to decide which factor model would fit the current data best and would be a suitable proposition in the conceptual debate on defensive style. All three explored factor solutions were interpretable. The final choice between the 3-, 4-, or 5-factor model was made on basis of the following aspects: the amounts of explained variance with PCA and with SCA, the range of item loadings, the mean item loading, the inter-item correlations and the item-remainder correlations of each factor, the internal consistency of a factor, the number of problematic items (item loading $<0.40,<0.20$ difference with other factors or both) and factor correlation matrices. A 5-factor solution turned out to be the theoretically and statistically best model and will be presented here like the original 3-factor structure was presented. The 5-factor model was then tested in a new sample of non-patients. 
Five-factor model of the DSQ The 5-factor model, as best fit for the DSO48 , consisted of 43 items; two items did not load over 0.20 on any factor and three items did not match with the factor's content. Factor I was named distrust and consists of 11 items describing distrust and suspicion in other people and that of other people towards oneself, whether or not with an aggressive notion. These items were all part of the original immature factor. Factor II was labelled self-protection. Seven items reflect a sense of trust in what lies ahead someone and describe manners to focus on retaining mental stability. Four items belonged previously to mature defenses, two items to immature defenses and one to neurotic defenses. Self-sacrifice refers to factor III. Ten items represent thoughts, feelings and behaviours which are not automatically leading up to someone's optimal state of well-being because other people interests are attended to, also when it appears not necessarily warranted or indicated. All these factor items were originally neurotic defense items. Factor IV is called avoidance. The nine items convey coping behaviors and thoughts that facilitate avoidance of difficult feelings or situations. Six items formerly belonged to the immature defense factor, two to neurotic defenses and one to mature defenses. Control is the fifth and last factor. These items show that being in control of everything can resolve difficult feelings or situations. Five out of six items were earlier mature defenses items and one item was an immature defense item

Table 5 contains statistics on the 5 -factor model's factor structure, homogeneity and internal consistency.

The SCA for the distrust, self-protection, self-sacrifice, avoidance and control factor structure could explain $37.7 \%$ of the variance in the total group with an acceptable $2.4 \%$ loss compared to the explorative 5-factor PCA. SCA explained variance in the separate groups was similar, although the loss to PCA averaged $5.9 \%$. Factor item loadings, inter-item correlations, item-remainder correlations and internal consistencies together appeared considerably better for the total and separate groups than for the immature, neurotic and mature factor structure. Following the relative relationships of correlations with other validation measures, the defense styles can be ranked from most pathological to healthy in the order distrust, avoidance, self-sacrifice, self-protection, and control. The correlation between self-esteem and self-protection, fits the ranking but the negative direction of the relationship is unexpected. Table 6 displays mean DSO 5factor scores with standard deviations and test statistics for the total group and for all separate groups. 
Table 5. Statistics of proposed 5-factor model of defensive styles for all groups.

\begin{tabular}{|c|c|c|c|c|}
\hline & $\begin{array}{c}\text { Mean item } \\
\text { loading (range) }\end{array}$ & Mean rii & Mean rir (range) & Alpha \\
\hline \multicolumn{5}{|l|}{ Total group $(n=282)$} \\
\hline I Distrust & $.65(.39 .81)$ & .36 & $.56(.26 .75)$ & .86 \\
\hline II Self-protection & $.51(.42 .61)$ & .13 & $.26(.16 .39)$ & .53 \\
\hline III Self-sacrifice & $.49(.34 .58)$ & .16 & $.32(.14 .42)$ & .66 \\
\hline IV Avoidance & $.61(.42 .70)$ & .29 & $.48(.25 .59)$ & .79 \\
\hline V Control & $.59(.49 .69)$ & .22 & $.36(.23 .49)$ & .62 \\
\hline \multicolumn{5}{|l|}{ BPD patients $(n=129)$} \\
\hline I Distrust & $.59(.37 .77)$ & .29 & $.49(.23 .69)$ & .82 \\
\hline II Self-protection & $.48(.39 .63)$ & .09 & $.20(.11 .22)$ & .44 \\
\hline III Self-sacrifice & $.52(.43 .59)$ & .19 & $.35(.26 .44)$ & .69 \\
\hline IV Avoidance & $.52(.37 .69)$ & .18 & $.34(.17 .54)$ & .66 \\
\hline V Control & $.54(.41 .68)$ & .14 & $.26(.11 .47)$ & .50 \\
\hline \multicolumn{5}{|c|}{ Cluster C PD patients $(n=55)$} \\
\hline I Distrust & $.54(.35 .71)$ & .23 & $.41(.16 .62)$ & .75 \\
\hline II Self-protection & $.41(.31 .48)$ & .03 & $.08(-.04 .18)$ & .19 \\
\hline III Self-sacrifice & $.39(.19 .52)$ & .07 & $.16(-.05 .31)$ & .39 \\
\hline IV Avoidance & $.59(.43 .69)$ & .26 & $.44(.26 .59)$ & .76 \\
\hline V Control & $.55(.46 .61)$ & .16 & $.27(.15 .39)$ & .51 \\
\hline \multicolumn{5}{|l|}{ Axis-I patients $(n=45)$} \\
\hline I Distrust & $.55(.25 .79)$ & .24 & $.42(.11 .67)$ & .75 \\
\hline II Self-protection & $.58(.48 .67)$ & .22 & $.38(.26 .49)$ & .67 \\
\hline III Self-sacrifice & $.43(.08 .61)$ & .09 & $.22(-.16 .44)$ & .52 \\
\hline IV Avoidance & $.51(.29 .67)$ & .17 & $.33(.07 .53)$ & .65 \\
\hline V Control & $.56(.38 .76)$ & .17 & $.31(.09 .58)$ & .56 \\
\hline \multicolumn{5}{|l|}{ Non-patients $(n=53)$} \\
\hline I Distrust & $.63(.19 .86)$ & .34 & $.53(.03 .79)$ & .81 \\
\hline II Self-protection & $.57(.39 .73)$ & .21 & $.36(.14 .57)$ & .64 \\
\hline III Self-sacrifice & $.49(.36 .58)$ & .16 & $.32(.17 .41)$ & .65 \\
\hline IV Avoidance & $.58(.49 .71)$ & .26 & $.44(.33 .58)$ & .75 \\
\hline $\mathrm{V}$ Control & $.59(.48 .72)$ & .23 & $.36(.20 .55)$ & .61 \\
\hline
\end{tabular}

Factor correlation matrix for total group

(above diagonal pearson $\mathrm{r}$, below diagonal pearson $\mathrm{r}$ corrected for attenuation)

\begin{tabular}{llllll}
\hline & I & II & III & IV & V \\
I Distrust & - & $.39 * *$ & $.47 * *$ & $.56^{* *}$ & $-.27^{* *}$ \\
II Self-protection & .57 & - & $.36 * *$ & $.39 * *$ & .06 \\
III Self-sacrifice & .63 & .61 & - & $.56^{* *}$ & $-.21^{* *}$ \\
IV Avoidance & .68 & .60 & .69 & - & $-.28^{* *}$ \\
V Control & -.37 & .11 & -.33 & -.41 & - \\
SCL-90 $(n=281)$ & $.65^{* *}$ & $.35^{* *}$ & $.44^{* *}$ & $.50^{* *}$ & $-.43^{* *}$ \\
\hline
\end{tabular}




\begin{tabular}{lccccc}
\hline BPDSI-IV $(n=239)$ & $.61^{* *}$ & $.29^{* *}$ & $.29^{* *}$ & $.46^{* *}$ & $-.37^{* *}$ \\
BPD Checklist $(n=247)$ & $.74^{* *}$ & $.34^{* *}$ & $.42^{* *}$ & $.51^{* *}$ & $-.43^{* *}$ \\
Self-Ideal Discr. $(n=226)$ & $.61^{* *}$ & $.25^{* *}$ & $.37^{* *}$ & $.66^{* *}$ & $-.53^{* *}$ \\
RSES $(n=245)$ & $-.35^{* *}$ & $-.17^{*}$ & $-.26^{* *}$ & $-.47^{* *}$ & $.45^{* *}$ \\
PDBQ-BPD $(n=278)$ & $.73^{* *}$ & $.37^{*}$ & $.53^{* *}$ & $.64^{* *}$ & $-.42^{* *}$ \\
IPO-lower level def. $(n=108)$ & $.77^{*}$ & $.45^{*}$ & $.51^{* *}$ & $.64^{* *}$ & $-.29^{*}$ \\
\hline
\end{tabular}

Note. $\mathrm{rii}=$ inter-item correlation; $\mathrm{r}_{\mathrm{ir}}=$ item-remainder correlation; ${ }^{*} P<.01,{ }^{*} P<.001 . \mathrm{SCL}-90$ : Symptom Checklist-90; BPDSI-IV: Borderline Personality Disorder Severity Index-IV; RSES: Rosenberg Self Esteem Scale; PDBO: Personality Disorder Belief Questionnaire; IPO-lower level: Inventory of Personality Organization - use of lower level defenses.

Table 6. Factor scores for each group for the 5 factor DSQ-43 items.

\begin{tabular}{lllllllll}
\hline & \multicolumn{2}{c}{$\begin{array}{c}\text { BPD } \\
\text { patients } \\
(\mathrm{N}=129)\end{array}$} & \multicolumn{2}{c}{$\begin{array}{c}\text { Cluster-C } \\
\text { patients } \\
(\mathrm{N}=55)\end{array}$} & \multicolumn{2}{c}{$\begin{array}{c}\text { Axis-I } \\
\text { patients } \\
(\mathrm{N}=45)\end{array}$} & \multicolumn{2}{c}{$\begin{array}{c}\text { Non-clinical } \\
\text { controls } \\
(\mathrm{N}=53)\end{array}$} \\
\hline Factor scores & Mean & $S D$ & Mean & $S D$ & Mean & $S D$ & Mean & $S D$ \\
I Distrust & $3.97^{\mathrm{a}}$ & 1.45 & $2.81^{\mathrm{b}}$ & 1.13 & $2.28^{\mathrm{b}, \mathrm{c}}$ & 0.97 & $1.88^{\mathrm{c}}$ & 0.96 \\
II Self-protection & $5.08^{\mathrm{a}}$ & 1.22 & $4.31^{\mathrm{b}}$ & 0.99 & $4.46^{\mathrm{b}}$ & 1.39 & $3.97^{\mathrm{b}}$ & 1.25 \\
III Self-sacrifice & $4.86^{\mathrm{a}}$ & 1.26 & $4.51^{\mathrm{b}}$ & 0.89 & $4.09^{\mathrm{b}, \mathrm{c}}$ & 1.05 & $3.74^{\mathrm{c}}$ & 1.06 \\
IV Avoidance & $5.39^{\mathrm{a}}$ & 1.31 & $4.91^{\mathrm{a}}$ & 1.46 & $3.60^{\mathrm{b}}$ & 1.23 & $3.22^{\mathrm{b}}$ & 1.27 \\
V Control & $3.61^{\mathrm{a}}$ & 1.29 & $3.60^{\mathrm{a}}$ & 1.26 & $4.14^{\mathrm{a}}$ & 1.36 & $5.39^{\mathrm{b}}$ & 1.41 \\
\hline
\end{tabular}

Note. Groups with a different suffix are significantly different from each other with at least $P<.05$.

BPD-patients can be significantly discriminated from all other groups with respect to distrust, self-protection, and self-sacrifice. Concerning the avoidance factor, BPD-patients differ significantly from Axis-I patients and non-patients but not from Cluster C PD patients. Cluster C PD patients differentiate themselves significantly from non-patients on distrust and self-sacrifice and also additional from Axis-I patients, when avoidance is considered. Non-patients distinguish themselves significantly from all other groups with respect to control.

\section{Cross validation in new sample}

Statistics of the proposed 5-factor model (of defensive styles) in a new sample of 472 non-patients are depicted in Table 7. The sample consisted of 472 persons without psychological complaints. Compared to the previous non-patient sample, the current group had about ten percent more women (339 females, 72\%), was on average four years younger in age (mean 32.45 years, SD 11.44), and the educational status was on average two levels higher (mean level 9.70, SD 1.43). 
Table 7. Statistics of proposed 5-factor model of defensive styles in new sample of nonpatients.

\begin{tabular}{lcccc}
\hline & $\begin{array}{c}\text { Mean item load- } \\
\text { ing (range) }\end{array}$ & Mean rii & $\begin{array}{c}\text { Mean rir } \\
\text { (range) }\end{array}$ & Alpha \\
\hline Non-patients $(n=472)$ & $.55(.33 .66)$ & .23 & $.39(.19 .53)$ & .71 \\
I Distrust & $.50(.43 .57)$ & .13 & $.25(.17 .33)$ & .52 \\
II Self-protection & $.50(.42 .59)$ & .17 & $.33(.23 .46)$ & .67 \\
III Self-sacrifice & $.54(.40 .69)$ & .20 & $.38(.19 .58)$ & .71 \\
IV Avoidance & $.56(.41 .66)$ & .18 & $.30(.13 .43)$ & .55 \\
V Control & & & & \\
\hline
\end{tabular}

Factor correlation matrix for total group

(above diagonal pearson $r$, below diagonal pearson $r$ corrected for attenuation)

\begin{tabular}{llllll}
\hline & I & II & III & IV & V \\
I & - & $.35^{* *}$ & $.37^{* *}$ & $.38^{* *}$ & -.05 \\
II & .57 & - & $.44^{* *}$ & $.34^{* *}$ & $.21^{* *}$ \\
III & .54 & .75 & - & $.39 * *$ & .07 \\
IV & .54 & .58 & .57 & - & -.07 \\
V & -.08 & .39 & .11 & -.11 & - \\
\hline
\end{tabular}

Note. $\mathrm{rii}=$ inter-item correlation; $\mathrm{rir}=$ item-remainder correlation; ${ }^{*} P<.001$.

The SCA for the 5 -factor model explained $31.60 \%$ of the variance in the total group with a $3.1 \%$ loss compared to the 5-factor PCA. Factor item loadings were good. As for the inter-item correlations, only 'distrust' and 'avoidance' were within the preferred range of .20-.40. Item-rest correlations were mostly on acceptable levels. Internal consistencies of 'distrust' and 'avoidance' were satisfactory, 'self-sacrifice' was almost satisfactory, whereas 'self-protection' and 'control' had insufficient Cronbach's alpha's.

\section{Discussion}

Aim of the present study was (1) to replicate the immature, neurotic and mature factor structure of the DSQ-36 with the DSQ-48, (2) to examine the psychometric properties of the DSQ-48, and (3) to compare defense styles of BPD patients with other patients and non-patients (discriminant validity).

The immature, neurotic and mature factor structure was replicated to the same level as Andrews, Pollock, et al. (1989) and Spinhoven, van Gaalen, et al. (1995). As intended, the internal consistency of the DSQ-48 neurotic factor was higher than of the DSQ-36 neurotic factor but the internal consistency of the mature factor was still insufficient. Further, evidence for concurrent, construct and, in part, discriminant validity was obtained. However, the psychometric limi- 
tations of the DSQ-36 were also replicated. Factorial homogeneity, internal consistency and in part discriminant validity were not satisfactory, as was the amount of variance explained with SCA. Although an immature, neurotic or mature defense style structure appears to have theoretical coherence and is wellknown and accepted throughout clinical practice, the statistical evidence for this structure is not solid. Taken previous and present DSQ-studies together (Andrews, Pollock, et al., 1989; Sammallahti \& Aalberg, 1995; Spinhoven, van Gaalen, et al., 1995; Trijsburg, van t' Spijker, Van, et al., 2000), one may hypothesize that the construct of defense mechanisms may be valid but that grouping in immature, neurotic and mature levels needs refinement. Other studies on defense style assessment, some using observer-rated measures, also illustrate that it is problematic to reach a well-defined order in defense styles based on maturity levels (Bond, Gardner, et al., 1983; Perry \& Cooper, 1989; Vaillant, 1976). A suggestion for adjustments in the interpretation of defense styles for this DSQ comes from the post-hoc analyses. The five factors of the defense style model that emerged from the post hoc analyses were distrust, selfprotection, self-sacrifice, avoidance, and control. The factors' order from most pathological to healthy appears to be distrust, avoidance, self-sacrifice, selfprotection and control, based on the correlations with other measures.

Compared to the 3-factor immature, neurotic and mature model, the suggested 5-factor model (like the explored 3- en 4-factor models) was able to explain a higher percentage of the total variance, had relatively larger factor item loadings, better inter-item and item-rest correlations, as well as more satisfying internal consistencies of the different factors. Correlations between factors and with validation measures also improved in the 5 -factor model. These observations held not only for the total group, but also for the separate groups. The level of discriminant validity for BPD-patients of the 5 -factor model was higher than for the 3-factor model. The tenor of the current findings is in line with other studies (Bond, Paris, et al., 1994; Devens \& Erickson, 1998; Lingiardi, Lonati, Delucchi, et al., 1999; Paris, Zweig-Frank, et al., 1996; Vaillant, 1994) demonstrating that BPD patients have stronger relations with the use of lower level defenses than less severe PD's as neurotic and/or Cluster C PD's have. Our results even indicated that the distrust, self-protection and self-sacrifice factors were able to distinguish BPD patients from Cluster $C$ patients, next to Axis-I patients and non-patient controls. Considering the content of the avoidance factor, being a core feature of Cluster C PD symptoms, one could already expect this factor not to differentiate between BPD and Cluster C PD's. 'Avoidance', on the other hand, proves to be able to distinguish PD patients from Axis-I patients and nonpatient controls.

A salient finding is that the Axis-I group could not be differentiated from the non-patient group by both the 3- and 5-factor defense style model, except for 
'control' of the 5 -factor model. This is especially interesting since psychiatric outpatients in the studies of Andrews, Pollock, et al. (1989), Spinhoven, van Gaalen, et al. (1995) and Trijsburg, van t' Spijker, et al. (2000) of apparently similar nature as the current Axis-I patients, could be discriminated from nonpatients in defensive style. A possible explanation can be the relatively low sample size of the Axis-I group. In this respect the axis-I patient group size constitutes a limitation of the present study. Another notable result is the clear discriminative power of the healthy control factor between non-clinical controls and patient groups.

Results of the 5-factor model replication in a large non-patient sample were less optimal as in the original non-patient sample. Compared to the previous results, the inter-item correlations and internal consistencies of the 'self-protection' and 'control' factors were somewhat disappointing. However, a possible explanation can be found in the relatively small number of items of these factor scales (respectively 7 and 6). The factor-item loadings of all factors, the inter-item and item-rest correlations of 'distrust', 'self-sacrifice' and 'avoidance' were satisfactory and comparable to earlier findings. When compared to the original mature, neurotic and immature DSO-structure, the replicated 5-factor model remains favourable over the 3 -factor structure. Unfortunately, no additional validation measures could be collected from the second non-patient sample. It was therefore not possible to see whether the ranking of factor scales with respect to psychopathology as obtained with the first sample could also be replicated.

Examining the DSO 5-factor scoring patterns within groups, it is striking that BPD patients have the highest scores of all groups on all factors but 'control'. Applicable to all four factors, a possible explanation is that the BPD characteristic tendency to think and act in extremes is reflected in the scores. This in contrast to other patients and non-patients who tend to think and act in a more nuanced way. The score of 'distrust' may seem relatively low compared to the other BPD factor scores while it's content (mainly splitting, acting out and projection) is generally viewed as most typical for BPD patients (e.g. Bond, Paris, et al., 1994; Lingiardi, Lonati, et al., 1999). Yet, of all groups BPD patients still have clearly the highest 'distrust' score, which means that they rely more on these defenses than others. The high BPD scores on 'avoidance' and 'selfprotection' can be understood in terms of BPD patients who are trying everything possible within their ability to avoid and/or block the intense mental suffering BPD patients are known to experience. The high 'self-sacrifice' score reflects BPD patients' dysfunctional willingness and efforts to achieve and maintain relationships with other people because of their abandonment fears. As expected, 'control', as the healthiest defense style, had the lowest score in the BPD group. The factor scoring patterns within the Cluster C PD group, Axis-I group and nonclinical group were as expected, fitting their psychopathological level and char- 
acteristics. With respect to content, do these interpretations further contribute to the validity of the 5 -factor model.

Another possible explanation for some BPD factor scores can be made referring to the DSQ self-report format. The DSQ assesses someone's defense style but it does not incorporate the appropriate application of defenses, including timing and extensiveness, in particular situations as is better possible with observer-rated methods. This observation also fits the moderate to low correlations between clinical, observer-rated defense style assessment and self-report defense style assessments (Perry \& Hoglend, 1998; Perry \& lanni, 1998) The 'self-protection' factor item 'when I have to face a difficult situation I try to imagine what it will be like and plan ways to cope with it' is used as illustration to this. The groups ratings on this item were similar $(P=.248)$. However, even though BPD patients may use this defense as much as other patients and nonpatients, considering the specific psychopathology of BPD patients, their actual imaginations and coping following this item will be different and unhealthier in nature than those of other groups but this cannot be read from the available DSQ scores (e.g. in preparing a job interview BPD patients will have a hard time listing their qualities with their average low self-esteem or have less objections to have some drink or take a few pills as part of their preparation). The item further illustrates how an initially mature defense item can have an 'immature' function. This may apply to many DSO items. It, therefore, not only touches the limitations of the self-report format but also the conceptual issue of maturity in defense styles.

We suggest to refer to the ranking of the 5-factor model as from most pathological to healthy (distrust, avoidance, self-sacrifice, self-protection and control), and not from immature, via neurotic, to mature. The maturity concept would be confusing since several defense mechanisms of the 3-factor model would then be oppositely classified in the 5-factor model. For example, the previously immature item 'I fear nothing' became part of the 'control' factor, which then would be a mature item. In the 5-factor model, the items and factors seem to be (general) coping mechanisms, without being specifically embedded in a psychodynamic framework. The correlations between the five factors and the PDBQ-BPD section (cognitive oriented beliefs), and between the five factors and the IPO-lower level defenses (psychoanalytical oriented) were almost identical, again making one wonder whether the concepts of cognitive coping strategies and defense strategies differ from each other or are largely overlapping.

The results of the present study lead to several suggestions for future research. First of all, it would be interesting to replicate the alternative 5-factor model (with 43 DSQ items) in new BPD-, Cluster C and Axis-I patient samples. Furthermore, the relation between this self-report DSO and other observer-rated defense style assessment methods (interview, vignettes, Q-sort) needs to be 


\section{Chapter 3}

clarified. The use of self-report and observer-rated measures next to each other, can be of value as an additional information source in the therapeutic process. Another relation to be disseminated is that between defense styles and cognitive coping strategies. Defense styles do play a large role in treatment processes and it would be worthwhile to examine the modifiability of defense styles. Results of an outpatient treatment study for BPD point into this direction (Giesen-Bloo, van Dijk, et al., 2006), but how sensitive defense styles to change are, and which variables are mediating or moderating this modification still needs to be determined.

\section{Conclusions}

The original structure of the DSQ of mature, neurotic and immature factors could only be replicated with psychometric shortcomings. A newly proposed 5factor structure of 'distrust', 'self-protection', 'self-sacrifice', 'avoidance' and 'control', fits the DSO (including 43 of the 48 items) statistically and interpretational better than the 3 -factor structure. Future research is recommended to replicate the 5-factor structure and to further disseminate the interpretation of defense styles. 


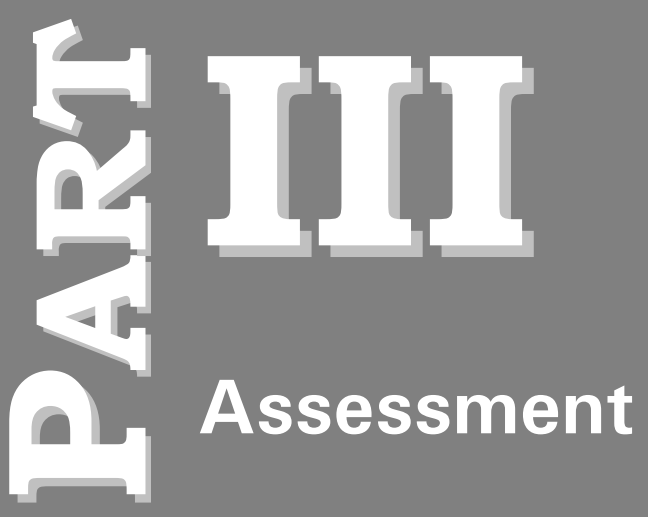





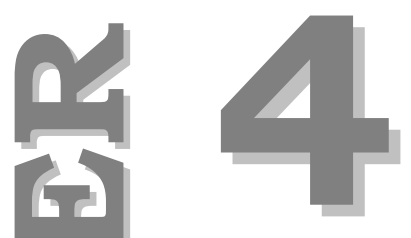

Assessment of Borderline Personality Disorder with the Borderline Personality Disorder Severity Index-IV: psychometric evaluation and dimensional structure 


\section{Abstract}

Background Debates on the (evaluative) assessment and conceptualization of Borderline Personality Disorder (BPD) are very much alive.

Aims To assess (psychometrical) quality of the Borderline Personality Disorder Severity Index - IV (BPDSI-IV) and to test different BPD-concepts in examining whether a one, a higher order or a DSM-multidimensional BPD concept is supported best.

Method The BPDSI-IV is a DSM-IV based semi-structured interview, designed to assess frequency and severity of BPD-specific manifestations during the past three months and developed for use as a treatment outcome measure. 242 subjects (108 BPD patients, 31 Cluster-C personality disorder patients, 27 patients with only Axis-I psychopathology and 76 non-patient controls) were interviewed with the BPDSI-IV. Additional data were obtained with self-report questionnaires. Seven multidimensional BPD-models were tested.

Results Internal consistency, discriminant, construct and concurrent validity proved to be very good. Clinical norms were derived and the instrument showed high sensitivity and high specificity. Confirmatory factor analyses supported both a one and 9-dimensional model based on the DSM-IV criteria. 


\section{Introduction}

Assessment of personality disorders (PDs) has been improved with the development of semi-structured interviews which have a long term perspective (Weertman, Arntz, Dreessen, et al., 2003; Zimmerman, 1994). However, the instability and fluctuations in symptoms of the borderline personality disorder (BPD) require an additional short term perspective. Another difficulty in assessing severity of BPD psychopathology is the conceptualisation of BPD. One major issue is whether or not BPD should be viewed as a one-dimensional or a multidimensional construct (Adams, Bernat, et al., 2001; Arntz, 1999; Clarkin, Hull, et al., 1993; Fossati, Maffei, Bagnato, et al., 1999; Hurt, Clarkin, et al., 1990; Livesley \& Schroeder, 1991; Morey, 1991; Sanislow, Grilo, et al., 2002; Zanarini, Gunderson, et al., 1989). Another issue is the categorical versus dimensional discussion. Implicit to a categorical approach is a considerable amount of heterogeneity, which accompanying problems are undesirable and argue for a dimensional view (Layden, Newman, et al., 1993; Lenzenweger \& Clarkin, 1996; Livesley, 2001). But, a categorical conceptualisation is often found to be more convenient in both clinical practice and empirical research.

The present study aims to assess the (psychometrical) quality of the Borderline Personality Disorder Severity Index - fourth version (BPDSI-IV). The interview covers a period of three months and is also developed for use as a treatment outcome measure. The BPDSI-IV is a DSM-IV based semi-structured interview, designed to assess the frequency and severity of manifestations, specific for BPD. Starting point was an adapted version of an interview developed by Weaver and Clum (1993). Psychometric properties of the BPDSI-I (1-year version) and BPDSI-II (3-months version) were very good and the instrument was sensitive to improvement during treatment (Arntz, van den Hoorn, et al., 2003). These results (Arntz, van den Hoorn, et al., 2003) and various practical arguments led to a clear distinction within the criterion 'unstable relationships' into 'partner relationships' and 'relationships with other people', the removal of two items, the formulation of seventeen new items and a more detailed explanation and guideline above each DSM-IV criterion for the interviewer. Clinical experiences and practical considerations were responsible for a further revision of the BPDSI-III into the BPDSI-IV. The criterion order of the BPDSI-IV is now identical to the order of the DSM-IV BPD criteria and a number of items were reformulated. The BPDSI-IV consists of 70 items, spread over nine criteria. For each item the frequency of the last three months is rated on an 11-point scale, running from 0 (never) to 10 (daily). Identity disturbance forms an exception since this item concerns a stable sense of self over a time period instead of a quantifiable symptom. Identity disturbance items are therefore rated on severity on a 5-point Likert scale, running from 0 (absent) to 4 (dominant, clear and well-defined not 
knowing who he/she is), the mean is multiplied with 2.5. Criteria scores for the 9 DSM-IV criteria are derived by averaging the item scores. The total score is the sum of the nine criteria scores (range 0-90). Moreover, the BPDSI-IV's structure lends itself for testing different BPD-concepts to examine whether a one-factor, a higher order factor or a DSM based eight or nine factor BPD concept is supported best. We compared seven dimensional BPD models.

\section{Method}

\section{Participants}

108 BPD-patients, 31 Cluster-C PD patients as a clinical control group with Axis-II psychopathology and 27 patients with only Axis-I psychopathology as a clinical control group with symptomatic psychopathology, were concurrently recruited from twelve mental health institutions (inpatient and outpatient). Participation to the study was voluntary and could be stopped at any time. 76 nonpatients were recruited through advertisements in local newspapers. Nonpatients received gift certificates, rated to the amount of time they participated in the study, with an average worth of 30 Euro. After a complete description of the study to all subjects, written informed consent was obtained. Diagnoses were assessed with DSM-IV based semi-structured interviews (SCID-I and II; First, Gibbon, et al., 1997; First, Spitzer, et al., 1996; Groenestijn, Akkerhuis, et al., 1999; Weertman, Arntz, et al., 2000). All subjects had to be between age 18 and 60. General exclusion criteria were psychotic disorders, bipolar disorder, psychiatric disorders secondary to medical conditions and mental retardation. BPD-patients were allowed to have comorbid personality and/or Axis-I disorders. Cluster-C patients had to meet criteria for at least one Cluster-C PD, were allowed to have comorbid Axis-I disorders but were not allowed to have an additional cluster-A or -B personality diagnosis or more than 2 BPD criteria. Patients with Axis-I psychopathology had to meet criteria for at least one Axis-I disorder, were not allowed to have threshold diagnoses of PD's or meet more than 2 BPD criteria. Non-patient controls had to be free of psychological complaints, could not have any Axis-I disorder, PD, PD threshold diagnosis or meet more than 2 BPD criteria.

\section{Measures}

After completing the diagnostic and BPDSI-IV interviews, the following instruments were administered: 
The BPD checklist (Giesen-Bloo, Arntz, et al., 2006) was added to assess self-reported severity of borderline related symptoms during the last month. Subjects indicated on a 5-point Likert scale from 'not at all' to 'extremely' how much they were troubled by 47 different complaints, derived from DSM-IV BPD criteria. The internal consistency in a BPD population proved to be excellent (Cronbach $\alpha=0.93$ ) (Giesen-Bloo, Arntz, van Dyck, et al., 2002).

The Personality Disorder Beliefs Questionnaire - BPD section (PDBQ) (Arntz, Dietzel, et al., 1999; Arntz, Dreessen, et al., 2004) is developed after cognitive theory of PD (Arntz, 1994b; Beck, Freeman, Davis, et al., 2004). It includes 20 beliefs, believed to be specific for BPD. The strength of each belief is rated on $100 \mathrm{~mm}$ visual analogue scales with "I don't believe this at all" on one end and "I believe this completely" on the other end. In a previous study (Arntz, Dietzel, et al., 1999), the PDBQ-BPD section showed excellent internal consistency (Cronbach $\alpha=0.95$ ).

The Rosenberg Self Esteem Scale (RSES) (Rosenberg, 1965; van den Hout, Arntz, \& Kunkels, 1995) consists of 15 items. Subjects indicate on a 6-point Likert scale from "completely true" to "not true at all" how much they agree with each statement. Internal consistency was found to be excellent (Cronbach's $\alpha=0.91$ ) (Arntz, van den Hoorn, et al., 2003).

Miskimins' self-goal-other discrepancy scale (Miskimins, Wilson, et al., 1971) assesses mean self-ideal and self-other discrepancies. 15 pairs of opposed personality characteristics have to be rated on $100 \mathrm{~mm}$ visual analogue scales from different points of view: how am I? (self), how do other people perceive me? (other) and how do I want to be? (ideal). The self-goal-other discrepancy scales had very good internal consistencies in the present study (Cronbach's $\alpha$ of self-other discrepancy $=0.86$, of self-ideal discrepancy $=0.89$ ) (Arntz, van den Hoorn, et al., 2003).

The Defense Style Questionnaire - 48 (DSQ-48) (Andrews, Pollock, et al., 1989; Giesen-Bloo, Arntz, Opdenacker, et al., 2006) is a psychoanalytically based self-report instrument, showing how subjects use mature, neurotic and immature defense mechanisms in daily life. 48 statements are rated on a 9-point Likert scale from "do not agree at all" to "do agree very much". Internal consistency of immature defenses proved to be very good in the present study population (Cronbach's $\alpha=0.88$ ).

The Symptom Checklist -90 (Arrindell \& Ettema, 1986; Derogatis, Lipman, et al., 1973) assesses general psychopathological complaints. Subjects rate to what extent they were bothered by 90 listed complaints during the last week on a 5-point Likert scale from "not at all" to "very much". The SCL-90 showed excellent internal consistency in the current sample (Cronbach's $\alpha=0.97$ ). 


\section{Tested multidimensional BPD concepts}

To examine whether a one, a higher order or a DSM-multidimensional BPD concept is supported best, seven multidimensional models of BPD were tested by means of the BPDSI-IV items. The appropriate BPDSI-IV/DSM-IV criteria, as we assigned them, are in parentheses after each model's factors. Because of the development of the DSM over time, not all DSM criteria of the BPDSI-IV could be appointed to a specific factor in every model. Therefore, only items relating to a particular factor of a model were used in the analysis, items of BPDSI-IV criteria not relevant for the model were omitted (see also data analysis section). The following BPD-models with the corresponding factors were included:

A. (Zanarini, Gunderson, et al., 1989) differentiated four scales of borderline pathology with the diagnostic interview for borderline patients (revised): affect $(6,7,8)$, cognition (9), impulsivity $(4,5)$ and interpersonal relationships $(1,2)$.

B. (Hurt, Clarkin, et al., 1990) identified three sets of BPD-criteria based on correlations between the DSM-III: identity $(1,3,7)$, affect $(2,6,8)$ and impulsivity $(4,5)$.

C. Morey, (1991; in Adams, Bernat, et al., 2001) developed the personality assessment inventory in which BPD is defined with four dimensions: affective instability $(6,8)$, identity problems $(3)$, negative relationships $(1,2)$ and self damaging behavior (5).

D. (Livesley \& Schroeder, 1991) reviewed the literature and examined which behaviours, of a pool of borderline related behaviours, fitted BPD patients best and differentiated them most from other cluster B personality disorder patients. They found three factors underlying BPD, namely their core BPD factor instability/disorganisation $(1,2,3,6,7,9)$ and the more associated factors interpersonal exploitation (no DSM-IV criteria applicable) and self damaging behavior $(4,5,8)$.

E. (Clarkin, Hull, et al., 1993) found evidence for a three factor structure in BPD in a study on the eight DSM-III-R criteria: uncertainty about self \& interpersonal difficulties $(1,2,3,7)$, affect $\&$ affect regulation $(5,6,8)$, and impulsivity (4).

F. (Clarkin, Hull, et al., 1993) further discussed on the value of anger/hostility as an independent fourth factor (8 separately from affect \& affect regulation).

G. (Sanislow, Grilo, et al., 2002) tested the DSM-IV BPD criteria as a unitary construct but also as a three-factor model comprising disturbed relatedness $(2,3,7,9)$, behavioral dysregulation $(4,5)$ and affective dysregulation $(1,6,8)$. 


\section{Raters}

The diagnostic and BPDSI-IV interviews were conducted by 21 extensively trained interviewers: nine psychologists/psychotherapists, one psychiatric nurse, two psychiatric research assistants and nine academic students who were finishing their masters thesis. One psychologist made second ratings of taped BPDSIIV interviews.

\section{Data analysis}

Internal consistencies of the total and criterion scores were satisfactory if Cronbach's $\alpha$ was above 0.70 (Nunnally, 1978). T-tests with planned comparisons or Mann Whitney tests were used to assess discriminant validity, as were Pearson (partial) correlations for concurrent and construct validity. Clinical norms were derived with formulas of Jacobson and Truax (Jacobson \& Truax, 1991).

To examine the dimensional structure of BPD, first order confirmatory factor analyses with structured equation modelling were executed with the BPDSIIV items fitted into the seven BPD models, and by calculating the Akaike information criterion (AIC; Akaike, 1987). Since only nested models can be statistically tested against each other, using the AIC is the best alternative. The AIC is a relative measure, able to compare different models that contain the same information. Every model had AIC's computed for the proposed three- or fourfactor structure, for a one-factor structure and for a so-called all factor structure, in which all DSM criteria of a model were taken as separate factors (six, eight or nine). The lowest AIC of comparable models indicates the most informative model. The three concepts of Hurt, Clarkin, et al. (1990) and both of Clarkin, Hull, et al. (1993) could be compared directly to each other, as were the two concepts of Livesley \& Schroeder (1991) and Sanislow, Grilo, et al. (2002). The models of Zanarini, Gunderson, et al. (1989) and Morey (1991) stood on themselves.

All tests were interpreted with a significance level of $5 \%$. Analyses were performed using the Statistical Package for Social Sciences, version 11.5 and LISREL version 8.30.

\section{Results}

\section{Group comparability}

Demographic data on the four groups are summarized in Table 1. Seventeen Axis-I patients had an anxiety disorder as primary diagnosis, 8 patients a mood disorder and 2 patients a somatoform disorder. 
Table 1. Demographic data, number of Axis-I diagnoses, number of Axis-II diagnoses without main diagnosis and number of BPD traits on all groups

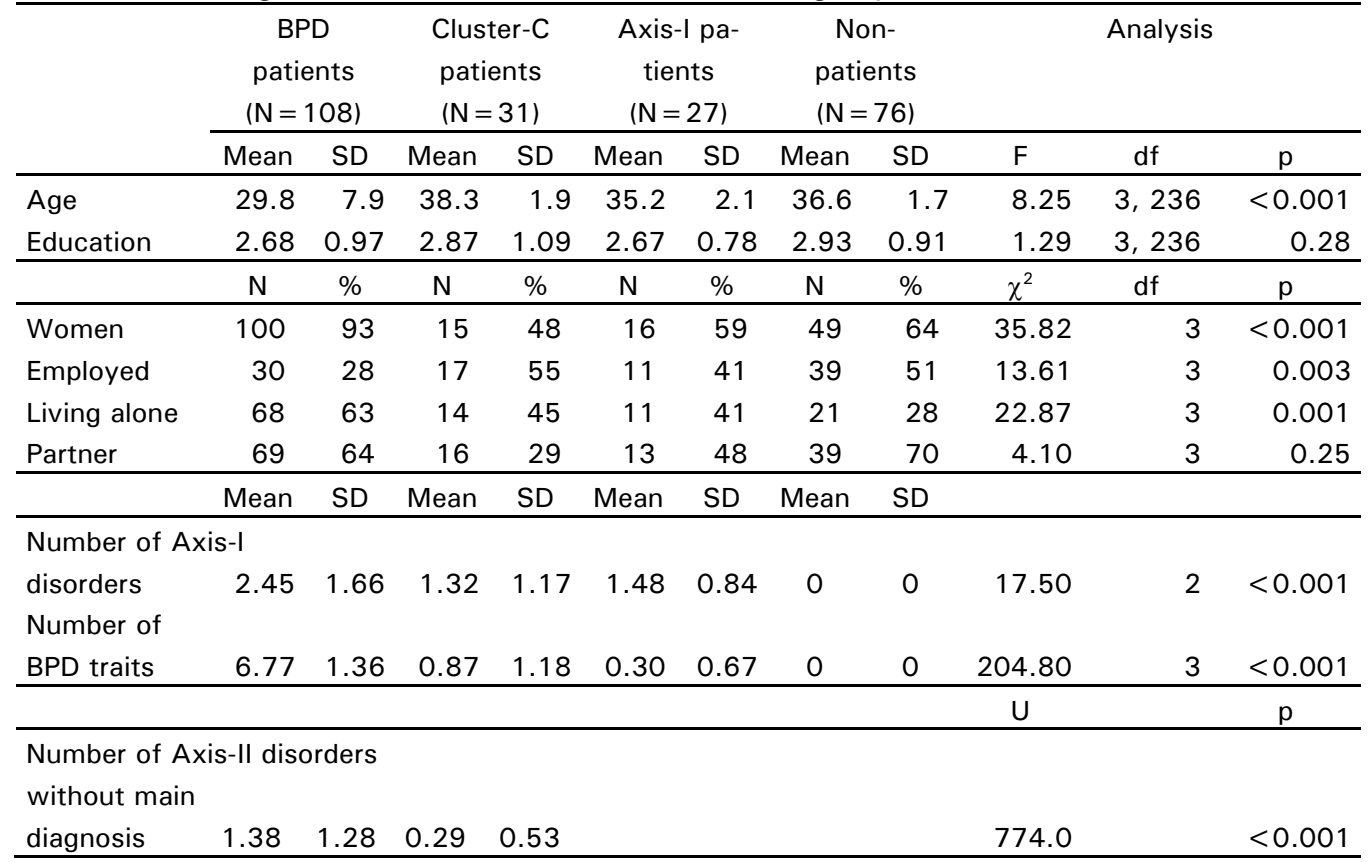

Note. ANOVA's for age and educational level; Chi-Square tests for gender, employment, living situation and partner; Kruskal-Wallis tests for number of Axis-I diagnoses and number of BPD traits; Mann-Whitney test for number of Axis-II diagnoses without main diagnosis.

The groups differed significantly with respect to age, gender, employment status, living situation, number of Axis-I diagnoses, number of Axis-II diagnoses without main diagnosis and number of BPD traits. No significant differences were found for educational level and having a partner. BPD-patients had on average significantly more Axis-I diagnoses than Axis-I patients $(U=927.50$, $p=0.003)$ and Cluster-C PD patients $(\mathrm{t}(68.6)=4.3, p<0.001)$. It was decided to correct further between-group analyses for these variables. Analyses on the BPD-patients were only corrected for number of Axis-I diagnoses as a regression analysis on the BPD group revealed a significant contribution of this variable to the BPDSI-IV score $(t=3.651, p<0.001,11.2 \%$ variance explained), the other variables were not significantly related to BPDSI-IV score within the BPD group.

\section{Interrater reliability}

Intraclass Correlation Coefficients (ICC's) of the BPDSI-IV total score and criterion scores were excellent $(0.98-1.00)^{1}$. ICC's for individual items ranged 
from 0.75 to 1.00 with the median at 1.00 . For five items no ICC could be computed due to lack of variance. ${ }^{1}$

\section{Internal consistency}

The internal consistency of the BPDSI-IV total score proved to be excellent in the total group (Cronbach's $\alpha=0.96$ ) and was very good for the BPD group (Cronbach's $\alpha=0.85$ ). Based on the total group, the internal consistency was also calculated for the separate cluster scores, or DSM criterion scores and ranged from satisfactory to excellent; avoids any abandonment $\alpha=0.79$, unstable relationships $\alpha=0.70$, identity disturbance $\alpha=0.89$, self-damaging impulsivity $\alpha=0.67$, recurrent (para)suicidal behavior $\alpha=0.81$, affective instability $\alpha=0.93$, chronic feelings of emptiness $\alpha=0.86$, lack of anger control $\alpha=0.80$, dissociation and paranoid ideation $\alpha=0.80$. Item-rest correlations ( $r_{i r}$ 's) of the item and criterion scores related to the total score ranged from zero to 0.88 (median items $=0.48$, median criteria $=0.78$ ) (Zimmerman \& Coryell, 1990). Removal of the items that appeared to have a negative influence on the internal consistency of the BPDSI-IV total score did not lead to such an improvement in internal consistencies of the involved criteria and BPDSI-IV total score that a removal was sufficiently warranted.

\section{Discriminant validity}

Table 2 presents BPDSI-IV mean total scores, mean DSM criterion scores and test statistics for all four groups.

BPDSI-IV total scores and DSM criterion scores of BPD-patients were all significantly higher than those of the control groups. Four items did not discriminate between BPD and other patients or non-patient controls, an additional 6 items could not differentiate between BPD- and Cluster-C patients and another 6 items were not able to separate BPD- from Axis-I patients. The problematic items, mainly situated in the DSM criteria 'unstable relationships', 'self-damaging impulsivity' and '(para)suicidal behavior', had very low frequencies in BPDpatients and were mostly dealing with the extreme behaviors of their DSMcriterion (e.g. new partners, hard drug use, sex with people you hardly know, sticking needles in your body).

\section{Concurrent and construct validity}

Pearson correlations and partial correlations of BPDSI-IV sum score and other instruments for the total group and the BPD group are depicted in Table 3. 
Table 2. BPDSI-IV mean total scores and mean DSM criterion scores of all groups.

\begin{tabular}{|c|c|c|c|c|c|c|c|c|c|c|}
\hline & \multirow[t]{2}{*}{ BPD } & \multirow[t]{2}{*}{ C-PD } & \multirow[t]{2}{*}{ Axis-I } & \multirow[t]{2}{*}{ NPCo } & \multicolumn{2}{|c|}{$\mathrm{BPD} \sim \mathrm{C}-\mathrm{PD}$} & \multicolumn{2}{|c|}{ BPD Axis-I } & \multicolumn{2}{|c|}{$\mathrm{BPD} \sim \mathrm{NPCo}$} \\
\hline & & & & & $t / U$ & $\mathrm{p}$ & $\mathrm{t} / \mathrm{U}$ & $\mathrm{p}$ & $\mathrm{t} / U$ & $\mathrm{p}$ \\
\hline Abandonment & 3.47 & 1.09 & 0.97 & 0.07 & 8.33 & $<0.001$ & 8.77 & $<0.001$ & 17.0 & $<0.001$ \\
\hline Relationships & 2.33 & 0.49 & 0.49 & 0.14 & 322.0 & $<0.001$ & 11.20 & $<0.001$ & 373.5 & $<0.001$ \\
\hline Identity dist. & 4.95 & 0.65 & 0.56 & 0.02 & 103.0 & $<0.001$ & 80.0 & $<0.001$ & 1.0 & $<0.001$ \\
\hline Impulsivity & 1.62 & 0.37 & 0.55 & 0.09 & 439.0 & $<0.001$ & 549.0 & $<0.001$ & 361.5 & $<0.001$ \\
\hline (Para)suicide & 1.15 & 0.29 & 0.15 & 0.00 & 736.5 & $<0.001$ & 535.0 & $<0.001$ & 864.5 & $<0.001$ \\
\hline Affective stab. & 6.96 & 2.79 & 1.20 & 0.32 & 7.06 & $<0.001$ & 12.14 & $<0.001$ & 111.5 & $<0.001$ \\
\hline Emptiness & 6.48 & 2.52 & 1.44 & 0.19 & 9.23 & $<0.001$ & 172.5 & $<0.001$ & 22.5 & $<0.001$ \\
\hline Anger-control & 3.00 & 1.25 & 0.73 & 0.36 & 703.5 & $<0.001$ & 338.0 & $<0.001$ & 523.5 & $<0.001$ \\
\hline Dissociation & 2.59 & 0.50 & 0.64 & 0.01 & 402.0 & $<0.001$ & 448.5 & $<0.001$ & 285.5 & $<0.001$ \\
\hline T o t a I & 32.50 & 9.95 & 6.73 & 1.20 & 13.96 & $<0.001$ & 40.0 & $<0.001$ & 0.00 & $<0.001$ \\
\hline
\end{tabular}

Table 3. Pearson correlations and partial correlations of the BPDSI-IV total score with other variables and test statistics.

\begin{tabular}{lcccc}
\hline & \multicolumn{2}{c}{ Total group } & \multicolumn{2}{c}{ BPD-patients } \\
\cline { 2 - 5 } & $r$ with BPDSI-IV & Corrected $r^{1}$ & $r$ with BPDSI-IV & Corrected $r^{2}$ \\
\hline \# DSM-IV BPD criteria & $0.89^{* * *}$ & $0.77^{* * *}$ & $0.33^{* * *}$ & $0.30^{* *}$ \\
BPD Checklist & $0.86^{* *}$ & $0.68^{* * *}$ & $0.54^{* * *}$ & $0.51^{* *}$ \\
PDBQ-BPD section & $0.77^{* *}$ & $0.56^{* * *}$ & $0.39^{* * *}$ & $0.40^{* *}$ \\
Self-other discrepancy & $0.21^{* *}$ & 0.03 & 0.09 & -0.01 \\
Self-ideal discrepancy & $0.58^{* * *}$ & $0.24^{* * *}$ & $0.38^{* * *}$ & $0.31^{* *}$ \\
DSQ-48 mature defenses & $-0.11^{* * *}$ & -0.10 & -0.03 & -0.04 \\
DSQ-48 neurotic defenses & $0.40^{* *}$ & $0.16^{*}$ & -0.10 & -0.14 \\
DSQ-48 immature def. & $0.62^{* *}$ & $0.35^{* * *}$ & $0.20^{+}$ & $0.18^{+}$ \\
SCL-90 & $0.81^{* *}$ & $0.58^{* * *}$ & $0.54^{* * *}$ & $0.48^{* * *}$ \\
Self-esteem & $-0.69^{* * *}$ & $-0.39^{* * *}$ & $-0.32^{* * *}$ & $-0.29^{* *}$ \\
\hline
\end{tabular}

Note. $+p<0.10 ;{ }^{*} p<0.05 ;{ }^{*}{ }^{*} p<0.01 ; * * * p<0.001$ (2-tailed). ${ }^{1}$ Corrected for age, gender, employment status, living situation, number of Axis-I diagnoses and number of Axis-II diagnoses without BPD; ${ }^{2}$ Corrected for number of Axis-I diagnoses.

The BPDSI-IV scores of the total group were strongly related, even after correction, to the hypothesized other variables. Initially significant correlations of the BPDSI-IV with self-other discrepancy and DSQ-48 mature defenses disappeared after correction for age, gender, employment status, living situation, number of Axis-I diagnoses and number of Axis-II diagnoses without BPD. Correlation with neurotic defenses was minimal. The BPDSI-IV also showed the hypothesized correlations in the BPD group, except for immature defenses $(p<0.10)$. Correction for number of Axis-I diagnoses led to minimal changes. 


\section{Clinical norms, sensitivity and specificity}

Clinical norms were derived with formulas of Jacobson and Truax (1991). Considering the BPD group, the dysfunctional cutoff score came to 14.93, indicating that people with a higher score very probably show BPD pathology. The functional cutoff score for these groups was 4.12, people with a lower score are probably nonpatients. The reference point between both cutoff scores, between BPD-patients and non-patient controls, is 5.66. Elaborating on the dysfunctional cutoff score, sensitivity was 0.97 (105 out of 108 BPD subjects were correctly classified) and specificity was 1 (76 out of 76 non-patients were correctly classified). For the functional cutoff score sensitivity was 1 (108 out of 108) and specificity 0.95 (72 out of 76). A reference point between the BPD group and the patient groups (Cluster-C and Axis-I) was 19.22. So, 19 distinguishes BPD-like psychopathology from cluster $C$ personality disorders.

The reference point between BPD patients and all other groups was 15.76. In sum, we propose 15 as clinical cutoff score for recovery from BPD, and 19 as inclusion criterion for BPD treatment trials.

\section{Dimensional structure}

First order confirmatory factor analyses using structured equation modeling were executed. The AIC's of the seven models and test statistics can be found in Table 4.

Each model explained the BPDSI-IV data, based on the AIC, significantly better with an all-factor structure than with the proposed multifactor or onefactor structures (all $P<.001$ ). So, the AIC's indicate that the gain of information using an all factor model outweighs the gain of using a three- or four-factor model. Furthermore, based on the AIC, Hurt's, et al. model (1990) extracted more information than both models of Clarkin, et al. (1993). Sanislow et al. model (2002) was similarly favorable over Livesley \& Schroeder (1991). Significance levels are not available because statistical tests are not allowed in comparing non-nested models. Noteworthy is the similarities between the most informative models of Hurt, et al. (1990) and Sanislow, et al. (2002): Hurt's 'impulsivity' and Sanislow's 'behavioral dysregulation' contain the same DSM-IV/BPDSI-IV criteria; Hurt's 'identity' and Sanislow's 'disturbed relatedness' (leaving out the 'dissociation' criterion due to absence in DSM-III-R classification of BPD) have two of the three criteria in common, and Hurt's 'affect' and Sanislow's 'affective dysregulation' also share two out of three criteria. 


\section{Chapter 4}

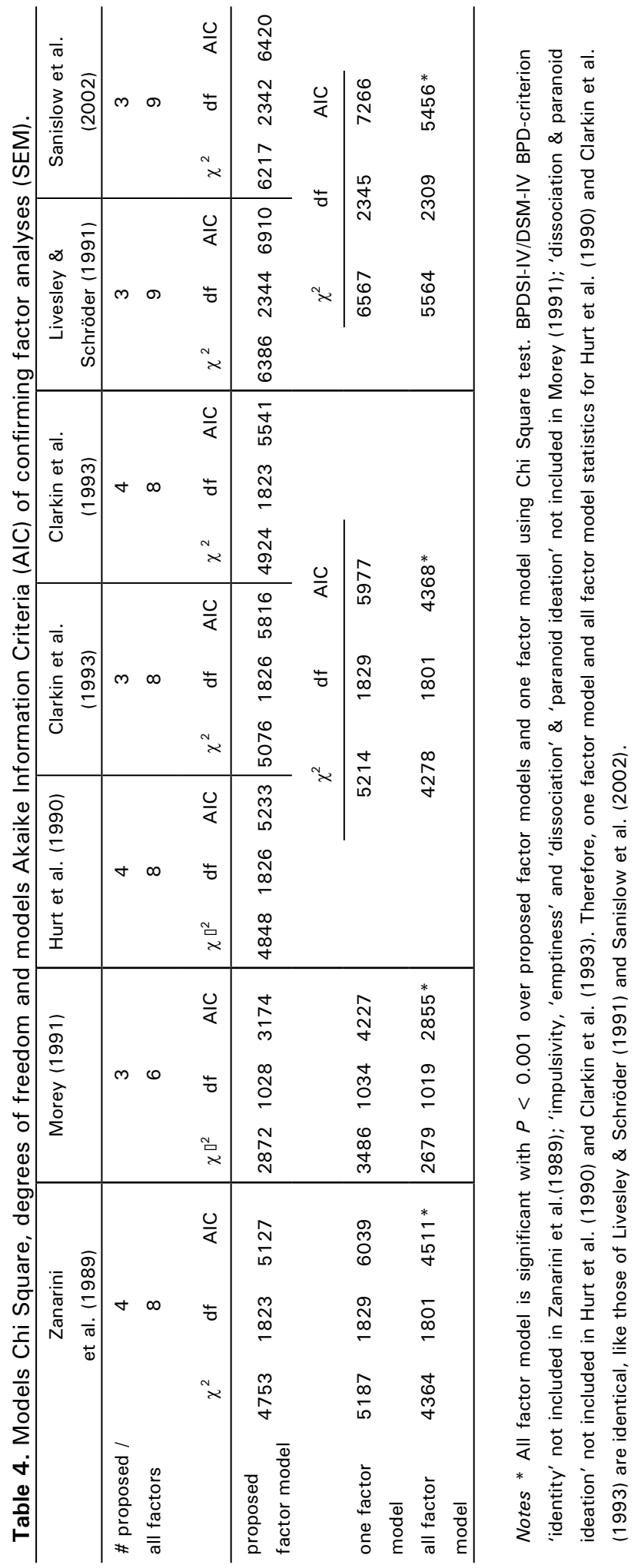




\section{Discussion}

It can be concluded that the BPDSI-IV proved to be a psychometrically sound instrument; the BPDSI-IV can assess severity and frequency of BPD specific psychopathology over a three-month period. The BPDSI-IV had excellent inter-rater reliability, satisfactory to excellent internal consistencies and very good discriminant, concurrent and construct validity. Clinical norms, specificity and sensitivity were derived for use throughout clinical practice and treatmentoutcome research (not for diagnosis since the BPDSI-IV is not in essence a diagnostic instrument). In addition, the BPDSI-IV has been used in a randomised controlled multicenter treatment study and proved to be sensitive to change (GiesenBloo, Arntz, et al., 2002; Giesen-Bloo, van Dyck, Spinhoven, et al., 2006).

Comparing the psychometric properties of the BPDSI-II (Arntz, van den Hoorn, et al., 2003) and the BPDSIIIV an overall improvement can be detected. The internal consistency of the total score (Cronbach's $\alpha$ ) for the total group and for the BPD group is again very satisfying (whole group: 0.96 versus 0.93 , and BPD group: 0.85 versus 0.82 ). For most DSM-IV BPD criteria the internal consistencies of the BPDSI-IV were higher than those of the BPDSI-II. Impulsivity is the only criterion with a marginal acceptable internal consistency of 0.67 for both the BPDSI-II and BPDSI-IV. An explanation could be that the eleven questioned impulsive behaviours are very typical for borderline psychopathology but also have a very divers nature (e.g. spending money, unsafe sex, reckless traffic participation, binge eating). A person's impulsive behaviour has often an idiosyncratic pattern (often between 4 or 6 of the 11 different items present, and null scores on the other items), making it unlikely that one individual scores on all impulsivity items. It is therefore probably too optimistic to expect that a higher internal consistency is possible. The discriminant, concurrent and construct validity for total and criterion scores were similar for BPDSI-II and BPDSI-IV. A possible limitation is the found influence of the number of axis-I diagnoses on the BPDSI-IV score. On the other hand this correlation might be spurious, as more severe BPD pathology might probably also lead to more Axis-I disorders.

Most adjustments that were made from the BPDSI-II to the BPDSI-IV seem satisfying, although a number of BPDSI-IV items were not able to differentiate between the groups.

The problematic items of the BPDSI-IV were mainly situated in the DSM criteria 'unstable relationships', 'impulsivity' and '(para)suicidal behaviors'. All problematic items had very low base rates in the BPD group. A possibility is that although these behaviours are specific for BPD, their occurrence is of very low frequency, so that they are not often exhibited in a 3-month period. But these items may also lack specificity to BPD. Further research is needed to elucidate this issue. 
The items 'sex with strangers' and 'shoplifting', having very low base rates in two studies, may be considered for removal in a next version of the BPDSI. Whether or not to remove items that fail to discriminate between groups is a delicate matter, which needs balancing statistics and theory. The other questionable items are thought to contain specific manifestations for BPD. Even though they do not meet psychometrical standards, theoretical grounds will probably prevent removal from a BPDSI-V. Future research will then have to prove the rightness of these decisions or show that reconsideration is indicated.

An issue for the 'relationships' criterion is its current internal structure, consisting of two parts with equal weight: 'partner relations' and 'relationships with other people'. However, when an individual does not have a partner, all questions for 'partner relations' were rated zero. This, in turn, may lead to an underestimation of the criterion 'relationships' and probably also that of the accompanying internal consistency.

The conclusions from the confirming factor analyses of seven different models depend on the application purposes of the BPD concept. If one wants to distinguish different classes within the BPD structure, we suggest to consider the nine DSM-IV criteria as nine factors, since our results imply that there seems to be no useful reduction of the DSM BPD criteria other than combining them into one BPD score. Compared to an all factor model (six, eight or nine), reduction to three or four factors (clusters of DSM-IV/BPDSI-IV criteria) led to considerable information loss. This part of the conclusion is not in line with a found three-factor structure as best differentiating and understanding BPD-structure by Sanislow, Grilo, et al. (2002), and can probably be explained by the use of the BPDSI-IV versus a structured interview for personality disorders. The BPDSI-IV checks the DSM-IV BPD criteria on a microscopic level with several items for each criterion, whereas a structured interview checks on a more general level, already filtering and clustering patient information to the (non)presence of a BPDcriterion. We found no convincing evidence for a model hypothesizing dimensions between the criterion level and the BPD-as-a-whole level. Thus, although the polythetic DSM model allows a wide variation in BPD criteria combinations leading to heterogeneity on the criterion level, the symptom level- the concept underlying the criteria appears to be one 'overall' dimension. Therefore, these results do support the primary conclusion by Sanislow, Grilo, et al. (2002) of BPD being a statistical unitary construct. Studies by Arntz (1999) and Fossati, Maffei, et al. (1999) also concur with such conclusion.

The relatively small sample size may be a limitation of this study, despite the large number of referring institutions. However, undermining this possible limitation are studies (Guadagnoli \& Velicer, 1988; MacCallum \& Widaman, 1999) that demonstrate the lack of validity of common rules of thumb for setting a sample size when executing factor analyses, and argue that absolute sample 
size is more important in determining the stability of a sample component pattern relative to the population pattern. Furthermore, the promising results with highly significant findings demonstrate a large power. Still, a larger sample size, especially in the clinical control groups, and extension to other clinical control groups (schizophrenia, cluster B personality disorders) would enlarge external validity. Future research should focus on further refinement of the BPDSI-interview, the discriminating power of the interview between different patient groups and its use as a treatment outcome measure.

${ }^{1}$ ICC-study with 26 mixed patients and nonpatients, five interviewers and one independent rater. A table with the ICC's, and also rir's, of individual, criterion and total scores can be requested from the author. 



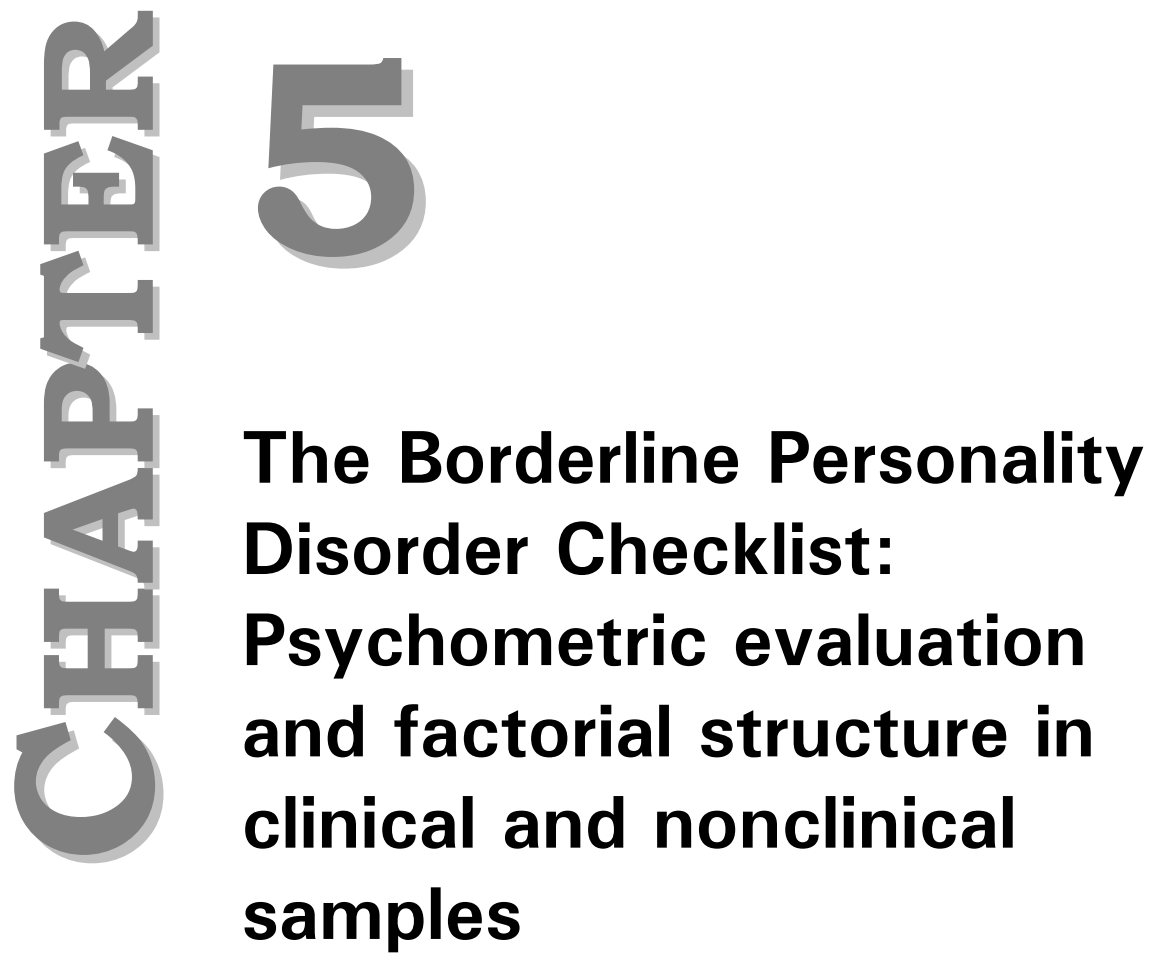

Giesen-Bloo, J., Arntz, A., Schouten, E. (Submitted for publication) 


\section{Chapter 5}

\section{Abstract}

The present study examined the assessment and conceptualization of borderline personality disorder with the Borderline Personality Disorder Checklist (BPD Checklist). The BPD Checklist is a DSM-IV based self-report questionnaire, designed to assess the experienced burden of specific BPD symptoms during the previous month. 140 BPD patients, 55 Cluster $C$ personality disorder patients, 57 patients with only Axis-I psychopathology and 87 nonclinical controls participated in the study. Psychometric properties and change during treatment of the BPD Checklist were evaluated. Internal consistency, construct, concurrent and discriminant validity proved to be very good. Clinical norms and cutoff scores with high sensitivity and specificity, were derived. The questionnaire is suitable as a screening instrument and treatment outcome measure since it proved to be sensitive to change. First order confirmatory factor analyses using the BPD Checklist items on seven dimensional BPD models supported both a one- and nine dimensional BPD model, the last being based on the DSM-IV criteria. 


\section{Introduction}

Borderline personality disorder (BPD) is a severe psychiatric condition, prevalent throughout health care settings, in which the instability in important life-areas (e.g. maintaining relationships, emotional regulation, impulsivity, identity and cognitive processes; APA, 1994) is the only constant factor. Clinical and research interests in BPD have expanded enormously since the DSMconceptualization of BPD. More or less parallel to this development is the increased understanding that the nature and complexity of BPD also requires specific screening and assessment instruments for both clinical and research purposes that aim to understand BPD and to reach evidence-based BPD treatment practice. Current common diagnostic interviews (e.g. SCID-II and DIB; First, Gibbon, et al., 1997; Zanarini, Gunderson, et al., 1989) often double as outcome measures in BPD (treatment) research (Brown, Newman, et al., 2004; Clarkin, Levy, Lenzenweger, et al., 2004; Hoglend, 1993; Nordahl \& Nysaeter, 2005; Stevenson \& Meares, 1992). In addition, interviews mostly cover long-term periods, are not designed to detect change in shorter time frames and above all, are very time consuming thus expensive. A further problem in investigating BPD with most of the widely used (self-report) outcome measures, is their focus on aspects of relatively general psychopathology, thereby only reflecting a part of the whole BPD-condition (e.g. general symptom levels, anxiety, depression, selfesteem). If compensated for in treatment evaluation by using a large set of instruments, time investment is again large.

Another difficulty in assessing severity of BPD psychopathology is the conceptualisation of BPD. One major issue is whether or not BPD should be viewed as an one-dimensional or a multidimensional construct (Hurt, Clarkin, et al., 1990; Morey, 1991; Zanarini, Gunderson, et al., 1989; Arntz, 1999; APA, 1994; Clarkin, Hull, et al., 1993; Fossati, Maffei, et al., 1999; Livesley \& Schroeder, 1991; Sanislow, Grilo, et al., 2002).

Two BPD specific instruments were developed in an outpatient general community mental health care setting with academic affiliations: the self-report BPD Checklist - 47 items questionnaire and the semi-structured borderline personality disorder severity index interview (BPDSI). Both instruments aim to assess the current severity of specific BPD manifestations and are designed for the screening and treatment evaluation of BPD patients. However, the BPD Checklist inquires for someone's experienced burden of BPD complaints during the last month, whereas the BPDSI forms a quantitative index of the severity and frequency of occurred BPD specific behaviors during the last three months. These instruments complement each other, e.g. by the self-report versus the interview format. Furthermore, the necessary time investment for the application of these measures is quite efficient. The relative short time frames make it also possible 
to map BPD characteristic instability. This paper addresses the properties of the BPD Checklist, the BPDSI is further described in Arntz, van den Hoorn, et al. (2003) and Giesen-Bloo, Wachters, et al. (2006). The BPD Checklist is a 47-item self-report questionnaire, based on therapists' clinical experiences, the relevant existing BPD literature and the BPD criteria of the DSM-IV. Items must be rated on a 5-point Likert scale, ranging from 'not at all' to 'extremely', indicating how much someone was troubled by the 47 different BPD complaints during the last month. The BPD Checklist's structure is similar to the DSM-IV BPD diagnostic criteria. Therefore, one can view the total sum score of the BPD Checklist or separate criterion sum scores.

The aim of the current study is (1) to assess the psychometric properties of the BPD Checklist, (2) to derive clinical norms, specificity and sensitivity, (3) to investigate the sensitivity to change and (4) to examine whether a one, a higher order or a DSM-multidimensional BPD concept is supported best when using the BPD Checklist items in testing seven dimensional models of BPD. Internal consistency, discriminant, concurrent and construct validity were examined in samples of BPD-patients, Cluster-C personality disorder patients, patients with only Axis-I psychopathology and non-clinical controls. It was hypothesized that the BPD Checklist would correlate positively with observed frequency and severity of BPD symptoms (BPDSI-IV; Arntz, van den Hoorn, et al., 2003; Giesen-Bloo, Wachters, et al., 2006), with specific BPD beliefs (PDBQ-BPD section; Arntz, Dietzel, et al., 1999; Arntz, Dreessen, et al., 2004), with maladaptive schemas (YSQ; Rijkeboer, van den Bergh, et al., 2005; Schmidt, Joiner, et al., 1995), with self-ideal discrepancy (self-goal-other discrepancy scale; Miskimins, Wilson, et al., 1971), with immature defense mechanisms (DSQ-48; Andrews, Pollock, et al., 1989; Giesen-Bloo, Arntz, et al., 2006), with pathological borderline personality organization features as formulated by Kernberg (IPO; Arntz \& Kerkhofs, 1999; Kernberg \& Clarkin, 1995; Lenzenweger, Clarkin, et al., 2001), and with general psychopathological complaints (SCL-90; Arrindell \& Ettema, 1986; Derogatis, Lipman, et al., 1973). A significant negative relation was expected between the BPD Checklist and self-esteem (RSES; Rosenberg, 1965). No substantial correlations with neurotic and mature defenses were expected. With respect to the different control groups, it was expected that BPD-patients' scores on the BPD Checklist would be significantly higher than either Cluster C PD patients, patients with Axis-I pathology or nonclinical controls. 


\section{Method}

\section{Participants}

140 BPD-patients, 55 Cluster C PD patients and 57 patients with only Axis-I psychopathology were concurrently recruited from twelve mental health institutions (inpatient and outpatient). Participation to the study was voluntary and could be stopped at any time. 87 nonclinical controls were recruited through advertisements in local newspapers. Nonclinical controls received gift certificates, rated to the amount of time they participated in the study, with an average worth of 30 euro. After a complete description of the study to all subjects, written informed consent was obtained. Diagnoses were assessed with DSM-IV based semi-structured interviews (SCID-I and II; First, Gibbon, et al., 1997; First, Spitzer, et al., 1996; Groenestijn, Akkerhuis, et al., 1999; Weertman, Arntz, et al., 2003; Weertman, Arntz, et al., 2000). All subjects had to be between age 18 and 60. General exclusion criteria were psychotic disorders, bipolar disorder, psychiatric disorders secondary to medical conditions and mental retardation. BPD-patients were allowed to have comorbid personality and/or Axis-I disorders. Cluster-C patients had to meet criteria for at least one Cluster-C PD, were allowed to have comorbid Axis-I disorders but were not allowed to have an additional cluster-A or -B personality diagnosis or more than 2 BPD criteria. Patients with Axis-I psychopathology had to meet criteria for at least one Axis-I disorder, were not allowed to have threshold diagnoses of PD's or meet more than 2 BPD criteria. Nonclinical controls had to be free of psychological complaints, could not have any Axis-I disorder, PD, PD threshold diagnosis or meet more than 2 BPD criteria.

\section{Measures}

After completing the diagnostic interviews in 1 or 2 sessions, the BPD Checklist and a series of instruments were administered in another session for convergent and discriminant validity of the BPD Checklist.

The Borderline Personality Disorder Severity Index-IV (BPDSI-IV; Arntz, van den Hoorn, et al., 2003; Giesen-Bloo, Wachters, et al., 2006). The BPDSI-IV is a DMS-IV BPD criteria based semi-structured interview and forms a quantitative index of the current severity and frequency of specific BPD manifestations. The interview covers a period of three months and is also developed for use as a treatment outcome measure. The BPDSI-IV consists of 70 items; spread over nine criteria in the same order as the DSM-IV. Each item is rated on an 11-point scale, running from 0 (never) to 10 (daily). Identity disturbance forms an exception since this concerns a stable sense of self over a time period instead of a 
quantifiable symptom. Therefore identity items are rated on a scale from 0 ('absent') to 4 ('dominant, clear and well-defined not knowing who he/she is'), the mean is then multiplied with 2.5. The total score is the sum of the nine averaged criteria scores (range 0-90). The index, but also the separate criterions, possess adequate reliability, as well as discriminant, concurrent and construct validity in similar patient groups and nonclinical controls (Arntz, van den Hoorn, et al., 2003; Giesen-Bloo, Wachters, et al., 2006).

The Personality Disorder Beliefs Questionnaire - BPD section (PDBO; Arntz, 1999; Arntz, Dreessen, et al., 2004). The PDBO is developed after cognitive theory of PD (Arntz, 1994b; Beck, Freeman, et al., 1990). The BPD-section includes 20 beliefs, believed to be specific for BPD. The strength of each belief is rated on $100 \mathrm{~mm}$ visual analogue scales with "I don't believe this at all" on one end and "I believe this completely" on the other end. Good internal consistency, criterion validity and stability of beliefs were demonstrated in Cluster A, B and C PD-patients, Axis-I patients and nonclinical controls (Arntz, 1999; Arntz, Dreessen, et al., 2004).

The Young Schema Questionnaire (YSQ; Rijkeboer, van den Bergh, et al., 2005; Schmidt, Joiner, et al., 1995). The YSO reflects 16 schemas after Young's extended cognitive theory of PD (Young \& Klosko, 1994). 205 items are rated on a 6-point Likert scale from "not true at all" to "totally true". Only a subsample of BPD patients $(n=81)$ filled out this questionnaire. The YSO proves to be highly sensitive in predicting the presence or absence of psychopathology (Rijkeboer, van den Bergh, et al., 2005). Internal consistency (Cronbach's alpha) of the YSO in the current sample was 0.98 .

The Rosenberg Self Esteem Scale (RSES; Rosenberg, 1965). The RSES consists of 15 items. Subjects indicate on a 6-point Likert scale from "completely true" to "not true at all" how much they agree with each statement. The scale has shown to possess good internal consistency, as well as construct, convergent and discriminant validity (Blascovich \& Tomaka, 1991; Rosenberg, 1965; 1979).

The Self-Goal-Other Discrepancy Scale (Miskimins, Wilson, et al., 1971). The self-goal-other discrepancy scale assesses mean self-ideal and self-other discrepancies. 15 pairs of opposed personality characteristics have to be rated on $100 \mathrm{~mm}$ visual analogue scales from different points of view: how am I? (self), how do other people perceive me? (other) and how do I want to be? (ideal). Only self-ideal discrepancy was administered in this study. The scale has adequate reliability, construct and discriminant validity (Berry, Miskimins, \& Wilson, 1972; Miskimins \& Baker, 1973; Miskimins, Wilson, et al., 1971).

The Defense Style Questionnaire - 48 (DSQ-48; Andrews, Pollock, et al., 1989; Giesen-Bloo, Arntz, et al., 2006). The DSQ-48 is a psychoanalytically based self-report instrument, showing how subjects use mature, neurotic and 
immature defense mechanisms in daily life. 48 statements are rated on a 9-point Likert scale from "do not agree at all" to " do agree very much". Reliability and validity (discriminant, concurrent \& construct) of the immature and neurotic scales are good, the mature scale is less robust (Giesen-Bloo \& Arntz, 2005; Spinhoven, van Gaalen, et al., 1995; Trijsburg, van t' Spijker, et al., 2000).

The Symptom Checklist -90 (SCL-90; Arrindell \& Ettema, 1986; Derogatis, Lipman, et al., 1973). The SCL-90 is a widely used self-report questionnaire, assessing general psychopathological complaints. Subjects rate to what extent they were bothered by 90 listed complaints during the last week on a 5-point Likert scale from "not at all" to "very much". Internal consistency of the global scale in the present sample was excellent (Cronbach's alpha $=0.97$ ). Convergent and discriminant validity were supported in an outpatient psychiatric population (Brophy, Norvell, \& Kiluk, 1988).

The Inventory of Personality Organization (IPO; Arntz \& Kerkhofs, 1999; Kernberg \& Clarkin, 1995; Lenzenweger, Clarkin, et al., 2001). The items of the IPO self-report questionnaire reflecting a borderline personality organizational structure were administered to a subsample of BPD patients $(n=78)$. The characterizing 90 items concern identity diffusion, use of lower level defenses, alterations in reality testing, pathological object relations (narcissistic and poor quality) and superego pathology, and were rated on a 5-point Likert scale from "never true" to "always true". Reliability and validity have been demonstrated in nonclinical samples (Lenzenweger, Clarkin, et al., 2001).

\section{Tested multidimensional BPD concepts}

To examine whether a one, a higher order or a DSM-multidimensional BPD concept is supported best, seven multidimensional models of BPD were tested by means of the BPD Checklist's items. The appropriate BPD Checklist / DSM-IV criteria, as we assigned them, are in parentheses after each model's factors. Because of the development of the DSM over time, not all DSM criteria of the BPD Checklist could be appointed to a specific factor in every model. Therefore, only items relating to a particular factor of a model were used in the analysis, items of BPD Checklist criteria not relevant for the model were omitted (see also data analysis section). The following BPD-models with the corresponding factors were included:

Zanarini, Gunderson, et al. (1989) differentiated four scales of borderline pathology with the diagnostic interview for borderline patients (revised version): affect $(6,7,8)$, cognition (9), impulsivity $(4,5)$ and interpersonal relationships $(1,2)$. 
Hurt, Clarkin, et al. (1990) identified three sets of BPD-criteria based on correlations between the DSM-III: identity $(1,3,7)$, affect $(2,6,8)$ and impulsivity $(4,5)$.

Morey (1991; in Adams, Bernat, et al., 2001) developed the personality assessment inventory in which BPD is defined with four dimensions: affective instability $(6,8)$, identity problems $(3)$, negative relationships $(1,2)$ and self damaging behavior (5).

Livesley \& Schroeder (1991) reviewed the literature and examined which behaviours, of a pool of borderline related behaviours, fitted BPD patients best and differentiated them most from other cluster B personality disorder patients. They found three factors underlying BPD, namely their core BPD factor instability/disorganisation $(1,2,3,6,7,9)$ and the more associated factors interpersonal exploitation (no DSM-IV criteria applicable) and self damaging behavior $(4,5,8)$.

Clarkin, Hull, et al. (1993) found evidence for a three factor structure in BPD in a study on the eight DSM-III-R criteria: uncertainty about self \& interpersonal difficulties $(1,2,3,7)$, affect $\&$ affect regulation $(5,6,8)$, and impulsivity (4).

Clarkin, Hull, et al. (1993) further discussed on the value of anger/hostility as an independent fourth factor (8 separately from affect \& affect regulation).

Sanislow, Grilo, et al. (2002) tested the DSM-IV BPD criteria as a unitary construct but also as a three-factor model comprising disturbed relatedness $(2,3,7,9)$, behavioral dysregulation $(4,5)$ and affective dysregulation $(1,6,8)$.

\section{Data analysis}

Internal consistencies of the total and criterion scores were deemed satisfactory if Cronbach's $\alpha$ was above 0.70 (Nunnally, 1978). T-tests with planned comparisons or Mann Whitney tests were used to assess discriminant validity, as were Pearson (partial) correlations for concurrent and construct validity. Clinical norms were derived with formulas of Jacobson \& Truax (1991). The sensitivity to change was tested with paired T-tests. To examine the dimensional structure of BPD, first order confirmatory factor analyses with structured equation modelling were executed with the BPD Checklist items fitted into the seven BPD models, and by calculating the Akaike information criterion (AIC; Akaike, 1987) Since only nested models can be statistically tested against each other, using the AIC is the best alternative. The AIC is a relative measure, able to compare different models that contain the same information. Every model had AIC's computed for the proposed three- or four-factor structure, for a one-factor structure and for a so-called all factor structure, in which all DSM criteria of a model were taken as separate factors (six, eight or nine). The lowest AIC of comparable models indicates the most informative model. The three concepts of Hurt, Clarkin, et al. (1990) and both of Clarkin, Hull, et al. (1993) could be compared 
directly to each other, as were the two concepts of Livesley \& Schroeder (1991) and Sanislow, Grilo, et al. (2002). The models of Zanarini, Gunderson, et al. (1989) and Morey (1991) stood on themselves.

All tests were interpreted with a significance level of $5 \%$. Analyses were performed using the Statistical Package for Social Sciences, version 11.5, and LISREL version 8.30.

\section{Results}

\section{Group comparability}

Demographic data, number of Axis-I diagnoses and number of BPD traits on the four groups are summarized in Table 1. Thirty-three Axis-I patients had an anxiety disorder as primary diagnosis, 18 patients a mood disorder, 4 patients a somatoform disorder and 1 patient a eating disorder. Twenty-eight Cluster C PD patients had an obsessive compulsive personality disorder, 24 patients an avoidant personality disorder and 3 patients a dependent personality disorder.

Table 1. Demographic data, number of Axis-I diagnoses and number of BPD traits on all groups

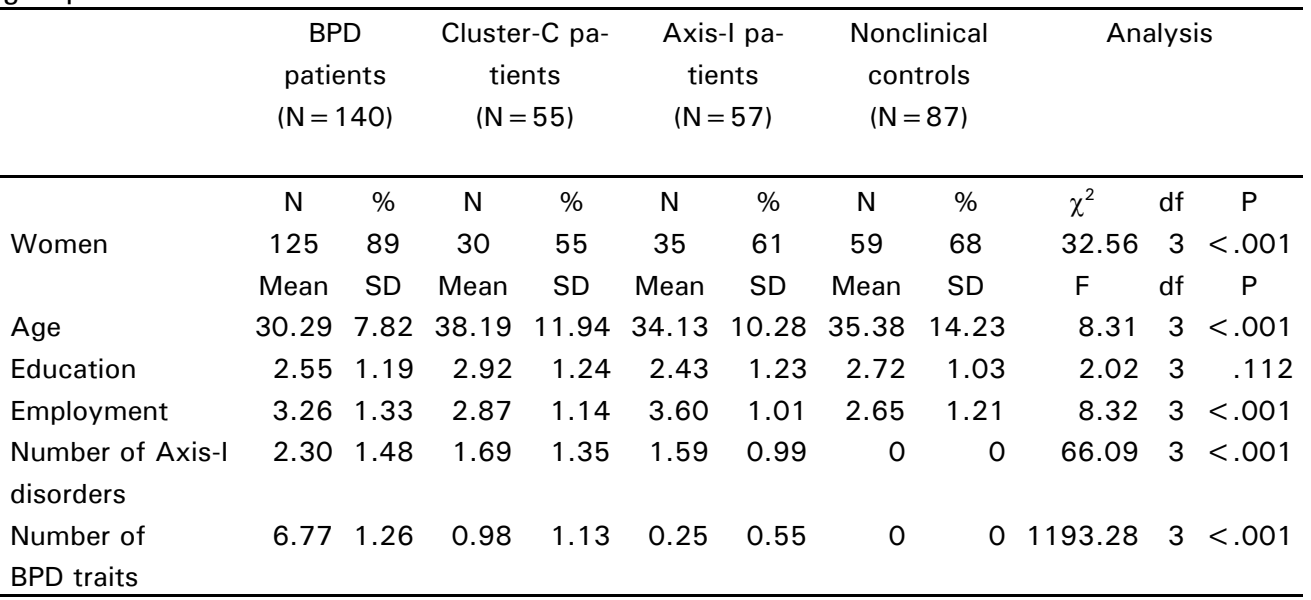

Note. Kruskal-Wallis test for gender; ANOVA's for age, educational level, employment status, number of axis-I disorders and number of BPD traits.

The groups differed significantly with respect to age, gender, employment status, number of Axis-I disorders and number of BPD traits. No significant difference was found for educational level. BPD-patients had on average significantly more Axis-I diagnoses than Axis-I patients $(U=2532, P=0.001)$. Therefore, further between-group analyses were corrected for these variables. Analy- 
ses on the BPD-group were only corrected for number of Axis-I diagnoses as a regression analysis on the BPD-group showed a single significant contribution of this variable to the BPD Checklist total score $(\mathrm{t}=2.16, P=0.033,12.0 \%$ variance explained).

\section{Internal consistency}

The internal consistency of the BPD Checklist proved to be excellent for both the total group and the BPD group (Cronbach's $\alpha$ respectively 0.97 and 0.92). Based on the total group, the internal consistency was also calculated for the separate cluster scores, or DSM criterion scores, and ranged from satisfactory to excellent; avoids any abandonment $\alpha=0.87$, unstable relationships $\alpha=0.78$, identity disturbance $\alpha=0.89$, self-damaging impulsivity $\alpha=0.69$, recurrent (para)suicidal behavior $\alpha=0.82$, affective instability $\alpha=0.93$, lack of anger control $\alpha=0.78$, dissociation and paranoid ideation $\alpha=0.86$. No $\alpha$ for chronic feelings of emptiness was calculated since this criterion is questioned through only one item, which correlated very well with the total score $\left(r_{i i}=0.84\right)$. Item-rest correlations $\left(r_{i r}{ }^{\prime} s\right)$ of the item, and of criterion scores related to the total score ranged from -0.01 to 0.90 (median items $=0.65$, median criteria $=0.84)^{1}$. Based on the item-rest correlations, two items appeared to have a negative influence: 'gambling' and 'shoplifting', both belonging to the self-damaging impulsivity criterion. Removal of these items improved the internal consistency of the selfdamaging impulsivity criterion to an acceptable homogeneity level (Cronbach's $\alpha=0.72$ ), the improvement in internal consistency of the total scale was negligible.

\section{Change during treatment}

Thirty-nine BPD patients participated in the uncontrolled pilot study of a large randomized controlled clinical treatment trial in which schema focused therapy and transference focused therapy were compared (SFT; Young \& Klosko, 1994; Young, Klosko, et al., 2003) (TFP, Clarkin, Yeomans, et al., 1999; Yeomans, Clarkin, et al., 2002). Therapy was open-ended and consisted of individual sessions twice a week. The BPD Checklist was administered threemonthly. With respect to the BPD Checklist, a six-month evaluation for 32 patients showed a significant average decrease of the total score with 14.00 points $(t(31)=2.64, P=.013$, two-tailed, $E S=.52)$. At one-year of SFT or TFP therapy, the total score of 26 patients dropped further with an average 24.27 points compared to baseline $(t(25)=3.58, P=.001$, two-tailed, $\mathrm{ES}=.95)$.

Eighty-eight BPD patients participated in the mentioned randomized controlled trial (Giesen-Bloo, van Dyck, et al., 2006). They also demonstrated large 
reductions in experienced BPD psychopathology as measured with the BPD Checklist (Giesen-Bloo, Arntz, et al., 2002). Six months after start of SFT or TFP therapy, 73 patients reduced their total BPD Checklist score with an average 17.34 points $(t(72)=6.24, P<.001$, two-tailed, $E S=.59)$. At one-year of therapy, the total score of 68 patients dropped further with an average 25.22 points compared to baseline $(t(67)=7.48, P<.001$, two-tailed, ES $=.83)$. The average BPD total score's reduction for 54 patients was 29.78 after two years of therapy $(t(53)=7.81, P<.001$, two-tailed, $E S=1.12)$.

\section{Discriminant validity}

Table 2 presents BPD Checklist mean total scores, mean DSM criterion scores with standard deviations and test statistics for all four groups.

Table 2. BPD Checklist mean total scores and mean DSM criterion scores of all groups.

\begin{tabular}{|c|c|c|c|c|c|c|c|c|c|c|}
\hline $\begin{array}{l}\text { Cluster } \\
\text { (score range) }\end{array}$ & $\begin{array}{l}\text { BPD } \\
\text { Mean } \\
\text { (sd) }\end{array}$ & $\begin{array}{l}\text { C-PD } \\
\text { Mean } \\
\text { (sd) }\end{array}$ & $\begin{array}{l}\text { Axis-I } \\
\text { Mean } \\
\text { (sd) }\end{array}$ & $\begin{array}{l}\text { NPCo } \\
\text { Mean } \\
(\mathrm{sd})\end{array}$ & $\begin{array}{l}\text { BPD - } \\
t / U\end{array}$ & $\begin{array}{l}\text { C-PD } \\
p\end{array}$ & $\begin{array}{l}\mathrm{BPD} \sim \\
\mathrm{t} / U\end{array}$ & $\begin{array}{c}\text { Axis-I } \\
p\end{array}$ & $\begin{array}{l}\text { BPD - } \\
U\end{array}$ & $\begin{array}{c}\text { NPCo } \\
p\end{array}$ \\
\hline $\begin{array}{l}\text { Abandonment } \\
\qquad(7-35)\end{array}$ & $\begin{array}{l}19.44 \\
(6.03)\end{array}$ & $\begin{array}{l}14.31 \\
(5.39)\end{array}$ & $\begin{array}{l}11.67 \\
(4.41)\end{array}$ & $\begin{array}{r}7.33 \\
(0.76)\end{array}$ & 2026 & $<0.001$ & 8.82 & $<0.001$ & 88.5 & $<0.001$ \\
\hline $\begin{array}{l}\text { Relationships } \\
\qquad(3-15)\end{array}$ & $\begin{array}{r}8.59 \\
(3.11)\end{array}$ & $\begin{array}{r}6.04 \\
(2.76)\end{array}$ & $\begin{array}{r}5.19 \\
(2.32)\end{array}$ & $\begin{array}{r}3.34 \\
(0.74)\end{array}$ & 2019.5 & $<0.001$ & 7.45 & $<0.001$ & 464 & $<0.001$ \\
\hline $\begin{array}{l}\text { Identity dist. } \\
(8-40)\end{array}$ & $\begin{array}{l}23.13 \\
(5.79)\end{array}$ & $\begin{array}{l}17.58 \\
(5.99)\end{array}$ & $\begin{array}{l}13.74 \\
(5.30)\end{array}$ & $\begin{array}{r}9.03 \\
(1.47)\end{array}$ & 5.87 & $<0.001$ & 10.57 & $<0.001$ & 119.5 & $<0.001$ \\
\hline $\begin{array}{c}\text { Impulsivity } \\
(9-45)\end{array}$ & $\begin{array}{l}15.39 \\
(4.23)\end{array}$ & $\begin{array}{l}12.04 \\
(3.69)\end{array}$ & $\begin{array}{l}11.04 \\
(2.82)\end{array}$ & $\begin{array}{r}9.76 \\
(1.20)\end{array}$ & 1830.5 & $<0.001$ & 1288.5 & $<0.001$ & 770 & $<0.001$ \\
\hline $\begin{array}{c}\text { (Para)suicide } \\
(3-15)\end{array}$ & $\begin{array}{r}5.85 \\
(3.03)\end{array}$ & $\begin{array}{r}3.98 \\
(1.59)\end{array}$ & $\begin{array}{r}3.39 \\
(1.03)\end{array}$ & $\begin{array}{r}3 \\
(0)\end{array}$ & 2247.5 & $<0.001$ & 1579 & $<0.001$ & 1609.5 & $<0.001$ \\
\hline $\begin{array}{l}\text { Affective } \\
\text { stab. } \\
\qquad(4-20)\end{array}$ & $\begin{array}{l}14.34 \\
(3.76)\end{array}$ & $\begin{array}{l}10.47 \\
(3.89)\end{array}$ & $\begin{array}{r}9.07 \\
(3.54)\end{array}$ & $\begin{array}{r}4.70 \\
(1.27)\end{array}$ & 6.31 & $<0.001$ & 9.09 & $<0.001$ & 83 & $<0.001$ \\
\hline $\begin{array}{l}\text { Emptiness } \\
\qquad(1-5)\end{array}$ & $\begin{array}{r}3.83 \\
(1.11)\end{array}$ & $\begin{array}{r}3.02 \\
(1.19)\end{array}$ & $\begin{array}{r}2.30 \\
(1.13)\end{array}$ & $\begin{array}{r}1.15 \\
(0.72)\end{array}$ & 4.35 & $<0.001$ & 8.71 & $<0.001$ & 273.5 & $<0.001$ \\
\hline $\begin{array}{l}\text { Anger-control } \\
\qquad(4-20)\end{array}$ & $\begin{array}{r}8.54 \\
(3.28)\end{array}$ & $\begin{array}{r}6.04 \\
(2.65)\end{array}$ & $\begin{array}{r}5.23 \\
(2.09)\end{array}$ & $\begin{array}{r}4.17 \\
(0.72)\end{array}$ & 1906.5 & $<0.001$ & 1338 & $<0.001$ & 731 & $<0.001$ \\
\hline $\begin{array}{c}\text { Dissociation } \\
(8-40)\end{array}$ & $\begin{array}{l}18.77 \\
(5.85)\end{array}$ & $\begin{array}{l}14.49 \\
(5.06)\end{array}$ & $\begin{array}{l}12.65 \\
(4.68)\end{array}$ & $\begin{array}{r}8.74 \\
(1.69)\end{array}$ & 4.77 & $<0.001$ & 7.04 & $<0.001$ & 277.5 & $<0.001$ \\
\hline $\begin{array}{l}\text { T o t a I } \\
(47-235)\end{array}$ & $\begin{array}{l}117.88 \\
(25.61)\end{array}$ & $\begin{array}{r}87.96 \\
(25.00)\end{array}$ & $\begin{array}{r}74.26 \\
(22.44)\end{array}$ & $\begin{array}{l}51.23 \\
(5.62)\end{array}$ & 7.47 & $<0.001$ & 11.22 & $<0.001$ & 20.5 & $<0.001$ \\
\hline
\end{tabular}

Note. C-PD = Cluster-C PD; NPCo $=$ Non-patient Controls; stab. =stability. T-tests of planned comparisons, with separate variance estimates or Mann-Whitney tests. 
Table 3. Pearson correlations and partial correlations of the BPD Checklist total score with other variables and test statistics.

\begin{tabular}{|c|c|c|c|c|}
\hline & \multicolumn{2}{|c|}{ Total group } & \multicolumn{2}{|c|}{ BPD-patients } \\
\hline & $r$ with BPD-CL & Corrected $r^{1}$ & $r$ with BPD-CL & Corrected $r^{2}$ \\
\hline \multirow[t]{2}{*}{ \# DSM-IV BPD criteria } & $0.75 * * *$ & $0.63 * * *$ & $0.27 * *$ & $0.22 *$ \\
\hline & $(n=325)$ & $(n=316)$ & $(n=132)$ & $(n=130)$ \\
\hline \multirow[t]{2}{*}{ BPDSI-IV } & $0.85 * *$ & $0.75 * * *$ & $0.56 * * *$ & $0.51 * * *$ \\
\hline & $(n=267)$ & $(n=263)$ & $(n=126)$ & $(n=124)$ \\
\hline \multirow[t]{2}{*}{ PDBQ-BPD section } & $0.67 * * *$ & $0.52 * * *$ & $0.39 * * *$ & $0.34 * * *$ \\
\hline & $(n=334)$ & $(n=312)$ & $(n=140)$ & $(n=130)$ \\
\hline \multirow[t]{2}{*}{ Self-ideal discrepancy } & $0.62 * * *$ & $0.43^{* * *}$ & $0.29 * *$ & $0.24 * *$ \\
\hline & $(n=287)$ & $(n=278)$ & $(n=127)$ & $(n=125)$ \\
\hline \multirow[t]{2}{*}{ DSO-48 mature defenses } & -0.09 & -0.05 & -0.55 & -0.04 \\
\hline & $(n=276)$ & $(n=271)$ & $(n=124)$ & $(n=122)$ \\
\hline \multirow[t]{2}{*}{ DSQ-48 neurotic defenses } & $0.49 * *$ & $0.35 * *$ & $0.30 * *$ & $0.28 * *$ \\
\hline & $(n=276)$ & $(n=271)$ & $(n=124)$ & $(n=122)$ \\
\hline \multirow[t]{2}{*}{ DSQ-48 immature def. } & $0.72 * * *$ & $0.62 * * *$ & $0.56 * * *$ & $0.54 * * *$ \\
\hline & $(n=276)$ & $(n=271)$ & $(n=124)$ & $(n=122)$ \\
\hline \multirow[t]{2}{*}{ SCL-90 } & $0.89 * * *$ & $0.81 * * *$ & $0.81 * * *$ & $0.79 * * *$ \\
\hline & $(n=292)$ & $(n=283)$ & $(n=126)$ & $(n=124)$ \\
\hline \multirow[t]{2}{*}{ Self-esteem } & $-0.75 * * *$ & $-0.64 * * *$ & $-0.58 * * *$ & $-0.57 * * *$ \\
\hline & $(n=280)$ & $(n=271)$ & $(n=131)$ & $(n=129)$ \\
\hline \multirow[t]{2}{*}{ YSO } & & & $0.79 * * *$ & $0.79 * * *$ \\
\hline & & & $(n=81)$ & $(n=81)$ \\
\hline \multirow{2}{*}{\multicolumn{2}{|c|}{ IPO-use lower level defenses }} & & $0.67 * * *$ & $0.68 * * *$ \\
\hline & & & $(n=78)$ & $(n=77)$ \\
\hline \multirow{2}{*}{\multicolumn{2}{|c|}{ IPO-identity diffusion }} & & $0.68 * * *$ & $0.69 * * *$ \\
\hline & & & $(n=78)$ & $(n=77)$ \\
\hline \multicolumn{3}{|l|}{ IPO-alterations reality testing } & $0.54 * * *$ & $0.54 * * *$ \\
\hline & & & $(n=78)$ & $(n=77)$ \\
\hline \multicolumn{3}{|c|}{ IPO-BPO pathological object relations } & $0.58 * * *$ & $0.58 * * *$ \\
\hline & & & $(n=77)$ & $(n=77)$ \\
\hline \multirow[t]{2}{*}{ IPO-superego pathology } & & & $0.56 * *$ & $0.58 * * *$ \\
\hline & & & $(n=78)$ & $(n=77)$ \\
\hline
\end{tabular}

Note. ${ }^{*} P<0.05 ;{ }^{*} P<0.01 ; * * * P<0.001$ (2-tailed). ${ }^{1}$ Corrected for age, gender, employment status and number of Axis-I diagnoses; ${ }^{2}$ Corrected for number of Axis-I diagnoses. Abbreviations: BPD-CL $=$ BPD Checklist, def. $=$ defenses.

BPD Checklist total scores and DSM-criterion scores of BPD-patients were all significantly higher than those of the control groups. Two items (gambling and shoplifting) did not discriminate between BPD and any control group, the same items responsible for the limited internal consistency of the criterion 'selfdamaging impulsivity'. They had very low frequencies in the BPD-patients. Three other items were not able to differentiate between BPD- and Cluster-C patients: identity item 'not daring to recognize the bad sides of yourself' $(P=0.057)$, dis- 
sociation item 'not being able to remember important things (not because of drugs)' ( $P=0.319$ ) and abandonment item 'frantically trying to prevent others from leaving you' $(P=0.118)$, although differences were all in the expected directions.

\section{Concurrent and construct validity}

Pearson correlations and partial correlations of BPD Checklist total score and other instruments for the total group and the BPD group are depicted in Table 3 .

The BPD Checklist scores of the total group were strongly related, even after correction, to the hypothesized other variables. The significant positive correlation with neurotic defenses deviated from the expected null correlation. Most correlations dropped slightly after correction for age, gender, employment status and number of Axis-I diagnoses but their discriminative power became more pronounced. The BPD Checklist also showed the hypothesized correlations in the BPD group. Correction for number of Axis-I diagnoses led to minimal changes.

\section{Clinical norms, sensitivity and specificity}

Clinical norms were derived with formulas of Jacobson \& Truax (1991). Considering the BPD group, the dysfunctional cutoff score came to 67.28, indicating that people with a higher score very probably show BPD pathology. The functional cutoff score, based on the non-clinical control group was 62.47, people with a lower score are probably non-patients. The reference point between both cutoff scores, between BPD-patients and non-patient controls, is 63.32. Elaborating on the dysfunctional cutoff score, sensitivity was 0.99 (138 out of 140 BPD patients were correctly classified) and specificity was 0.98 (85 out of 87 non-patients were correctly classified). For the functional cutoff score sensitivity was 1 (140 out of 140) and specificity 0.94 (82 out of 87). A reference point between the BPD group and the patient groups (Cluster-C and Axis-I) was 99.23. So, scores above 100 signify BPD-like psychopathology.

In sum, we propose 67 as clinical cutoff score for recovery from BPD, and 100 as indicative of BPD and inclusion criterion for BPD treatment trials.

\section{Factorial structure}

First order confirmatory factor analyses using structured equation modeling were executed. The AIC's of the seven models and test statistics can be found in Table 4. 
Chapter 5

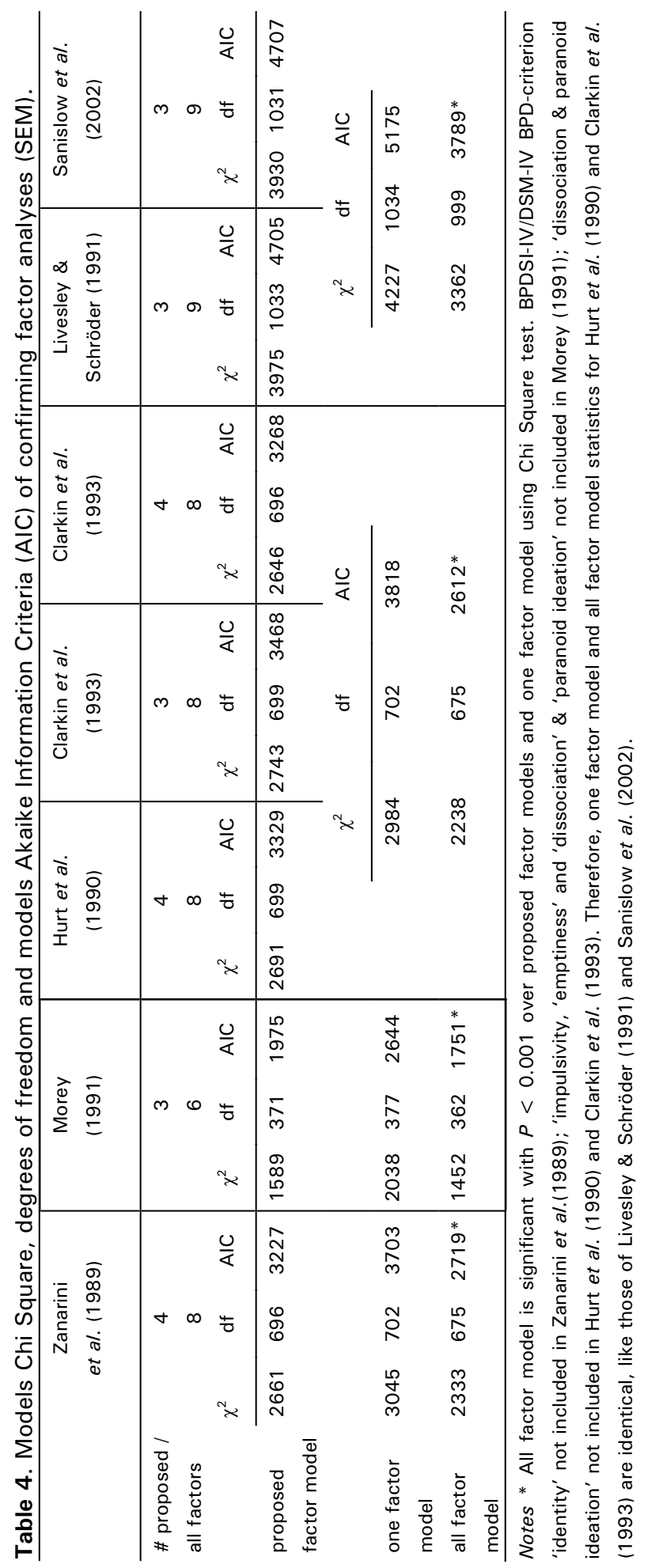


The all-factor models (meaning models with DSM-IV criteria as factors) explained the BPDSI-IV data significantly better, based on the AIC, than the proposed multifactor or one-factor structures (all $P<.001$ ). So, the AIC's indicate that the gain of information using an all factor model outweighs the gain of using a three- or four-factor model. Furthermore, based on the AIC, Clarkin, Hull, et al. 's 4-factor model (1993) extracted more information than their 3-factor model (1993) and that of Hurt, Clarkin, et al. (1990). Sanislow's model (2002) and that of Livesley \& Schroeder (1991) do equally well. Significance levels for betweenrow comparisons in Table 2 are not available because statistical tests are not allowed in comparing non-nested models.

\section{Discussion}

This study examined the BPD Checklist with psychometrical methods for suitability as a screening instrument and/or a (treatment) outcome measure and aimed to contribute to an increase in understanding of the conceptualization of BPD.

The internal consistency of the total and criterion scores was mostly very satisfying. Only the 'self-damaging impulsivity' criterion was marginally acceptable. However, removing two of the nine criterion-items, 'gambling' and 'shoplifting' improved this internal consistency from $\alpha=0.69$ to $\alpha=0.72$. Another supporting argument would be that these items were also problematic with respect to the discriminating power between BPD patients and other (non) patients. On the other hand, both items had very low base rates in the BPD group. A possible explanation could be that, although the nature of these behaviors is specific for BPD, their occurrence is of very low frequency, so that they are not often exhibited in a 1-month period. But, these low frequencies may also indicate that these behaviours are not very common in BPD and therefore not prototypical of BPD symptomatology. Two psychometric studies of the BPDSI (Arntz, van den Hoorn, et al., 2003; Giesen-Bloo, Wachters, et al., 2006) dealt with similar problems for it's items concerning gambling and shoplifting. Following these repeated findings on 'gambling' and 'shoplifting', we suggest to leave these symptoms aside the future DSM conceptualizations of BPD. The lack of discriminant ability between BPD patients and Cluster C PD patients of one abandonment, one identity and one dissociation item cannot be explained by low frequencies in the BPD group. Whether or not to remove items remains a delicate matter, which needs the balancing of statistics and theory. In our opinion, removal of these items is for theoretical reasons not yet fully warranted. Moreover, the contribution of each item towards the corresponding DSM-criterion 
score or total score was not disproportionably. Further research on the BPD Checklist will have to demonstrate the appropriateness of this decision.

The interpretation of the confirming factor analyses of seven different models depend on the application purposes of the BPD concept. If one wants to distinguish different classes within the BPD structure, we suggest to consider the nine DSM-IV criteria as nine factors, since our results imply that there seems to be no useful reduction of the DSM BPD criteria other than combining them into one BPD score. Compared to an all factor model (six, eight or nine), reduction to three or four factors (clusters of DSM-IV criteria) led to considerable information loss. This observation is not in line with a found three-factor structure as best differentiating and understanding BPD-structure by Sanislow, Grilo, et al. (2002), and may be related to the use of a self-report questionnaire versus a structured interview for personality disorders. The BPD Checklist checks the DSM-IV BPD criteria on a microscopic level with several items for each criterion (except for emptiness), whereas a structured interview checks on a more general level, already filtering and clustering patient information to the (non-)presence of a BPDcriterion. We found no convincing evidence for a model hypothesizing dimensions between the criterion level and the BPD-as-a-whole level. Thus, although the polythetic DSM model allows a wide variation in BPD criteria combinations leading to heterogeneity on the criterion level - the concept underlying the criteria appears to be one 'overall' dimension. Therefore, these results do support the primary conclusion by Sanislow, Grilo, et al. (2002) of BPD being a statistically unitary construct. Studies by Arntz (1999) and Fossati, Maffei, et al. (1999) also concur with this view. Most striking with respect to the current findings, is the resemblance with the factor results of a study on the BPDSI-IV (Giesen-Bloo, Wachters, et al., 2006) with a similar design, including the same seven BPD models. The favourableness of a so-called all-factor BPD model (based on the DSM) over the proposed or a one-factor model was almost exactly replicated in this study. Only two differences can be noted within the proposed factor models AIC's: (1) the 4-factor model of Clarkin, Hull, et al. (1993) and not the 3-factor model of Hurt, Clarkin, et al. (1990), explained the data best in the present study and (2) the model of Sanislow, Grilo, et al. (2002) was not superior over Livesley \& Schroeder (1991), but the data was explained equally well. The mentioned differences in proposed factor models illustrate that creating different factors with different labels, which are then viewed on themselves, do not necessarily provide additional information/understanding of an individual compared to a one or an all-factor model. The choice for using a one, a proposed of an all-factor BPD model depends on its aim and/or setting for which specific (BPD-) information is required.

As noted in the introduction section, the BPD Checklist and the BPDSI are complementary to each other. By now, both instruments have proven to comply 
to psychometric standards. It will be interesting to examine different manners of determining improvement (or worsening) of BPD-patients during therapy: the clinical perspective of one's therapist, a person's experienced change (the BPD Checklist), and a person's objective change (the BPDSI). As interesting will be to investigate whether different measures of change keep in step with each other. If possible, it is therefore desirable to administer the BPD Checklist and the BPDSI, at the same assessments. Preliminary results of BPD Checklist and BPDSI scores in a BPD treatment trial indicate that observed improvement in frequency of BPD manifestations precedes the experienced improvement in their burden (Giesen-Bloo, Arntz, et al., 2002)

\section{Conclusions}

It can be concluded that the BPD Checklist proved to be a psychometrically sound instrument. The BPD Checklist had good to excellent internal consistencies and very good discriminant, convergent and construct validity. The BPD Checklist proved to be sensitive to change since the experienced BPD specific symptoms significantly decreased during psychotherapeutic treatments. First order confirmatory factor analyses supported both a one- and a nine dimensional model based on the DSM-IV BPD criteria. Clinical norms, specificity and sensitivity were derived for use in clinical practice and in research. We proposed 67 as clinical cutoff score for recovery from BPD when the BPD Checklist is used as treatment outcome measure. To indicate BPD patients, for example in screening/inclusion procedures before BPD treatment trials, a cutoff score of 100 is suggested.

Future research should focus on the functioning of the BPD Checklist as screening instrument and as treatment outcome measure. Further solidifying the BPD Checklist's validity, including other clinical control groups (e.g. cluster B personality disorders, schizophrenia) is also indicated.

${ }^{1} A$ table with the item-rest correlations of individual, criterion and total scores can be requested from the author. 



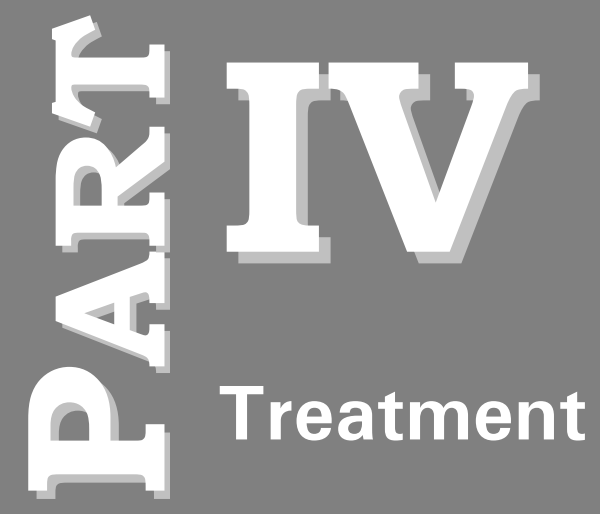





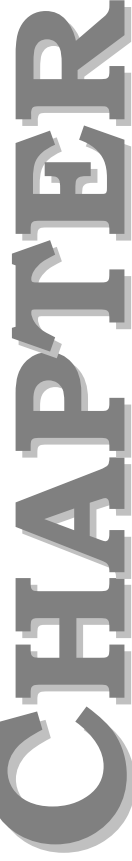

\section{Outpatient Psychotherapy} for Borderline Personality Disorder: A randomized trial of Schema focused therapy versus Transference focused psychotherapy

Giesen-Bloo, J., van Dyck, R., Spinhoven, P., van Tilburg, W., Dirksen, C., van Asselt, T., Kremers, I., Nadort, M., Arntz, A. (2006). Archives of General Psychiatry, 63, 649-58. 


\section{Abstract}

Context Borderline Personality Disorder is a severe and chronic psychiatric condition, prevalent throughout health care settings. So far, of current treatments only limited effects have been documented.

Objective To compare the effectiveness and relative effectiveness of schema-focused therapy (SFT) and psychodynamically based transference-focused psychotherapy (TFP) in patients with borderline personality disorder.

Design A multicenter, randomized 2-group design.

Setting Four general community mental health centers.

Participants Eighty-eight patients with a Borderline Personality Disorder Severity Index, fourth version, score greater than a predetermined cutoff score.

Intervention Three years of either SFT or TFP with sessions twice a week.

Main Outcome Measures Borderline Personality Disorder Severity Index, fourth version, score; quality of life; general psychopathologic dysfunction; and measures of SFT/TFP personality concepts. Patient assessments were made before randomization and then every 3 months for 3 years.

Results Data on 44 SFT-patients and 42 TFP-patients were available. The sociodemographic and clinical characteristics of the groups were similar at baseline. Survival analyses revealed a higher dropout risk for TFP-patients than for SFT-patients $(P=0.01)$. Using an intention-to-treat approach, statistically and clinically significant improvements were found for both treatments on all measures after a 1-, 2- and 3-year treatment periods. After 3 years of treatment, survival analyses demonstrated that significantly more SFT-patients recovered (relative risk $=2.18 ; P=.04$ ) or showed reliable clinical improvement (relative risk $=2.33 ; P=.009$ ) on the Borderline Personality Disorder Severity Index, fourth version. Robust analysis of covariance (ANCOVA) showed that they also improved more in general psychopathologic dysfunction and measures of SFT/TFP personality concepts $(P<.001)$. Finally, SFT-patients showed greater increases in quality of life than TFP-patients (robust ANCOVAs, $P=.03$ and $P<.001$ ). 
Conclusion Three years of SFT or TFP proved to be effective in reducing borderline personality disorder-specific and general psychopathologic dysfunction and measures of SFT/TFP concepts and in improving quality of life; SFT is more effective than TFP for all measures. 


\section{Introduction}

Borderline personality disorder (BPD) is marked by chronic instability in multiple areas (ie, emotional dysregulation, self-harm, impulsivity and identity disturbance). The prevalence of BPD is estimated to be $1 \%$ to $2.5 \%$ in the general population and $10 \%$ to $50 \%$ in psychiatric outpatient and inpatient settings (APA, 1994). The medical and other societal costs for BPD are substantial (Ten Have, Lorsheyd, et al., 1995; van Asselt, Dirksen, et al., 2006). Suicide risk is estimated to be up to $10 \%$ (Paris, 1993). A few treatments - outpatient dialectical behavior therapy (Lieb, Zanarini, et al., 2004; Linehan, Armstrong, Suarez, et al., 1991; Linehan, Heard, \& Armstrong, 1993; Linehan, Schmidt, Dimeff, et al., 1999; Verheul, van den Bosch, et al., 2003) and psychoanalytically oriented treatments (Bateman \& Fonagy, 1999; 2001; Munroe-Blum \& Marziali, 1995) have demonstrated some effectiveness in randomized clinical trials of patients with BPD, as manifested by good treatment retention, and reduced suicide attempts, acts of self-harm, and hospitalizations. However, no pharmacological or psychosocial treatment has demonstrated efficacy for all aspects of BPD, such as affective, identity and interpersonal disturbances (APA, 2001)

We compared the effectiveness of 2 prolonged outpatient treatments that aim at achieving full recovery from BPD: schema-focused therapy (SFT; Young, 1999; Young, Klosko, et al., 2003) and transference-focused psychotherapy (TFP; Clarkin, Yeomans, et al., 1999; Yeomans, Clarkin, et al., 2002). Schemafocused therapy is an integrative cognitive therapy, and TFP is a psychodynamically based psychotherapy. Both treatments intend to bring about a structural change in patients' personality, which should be apparent not only from a decrease in self-destructive behaviors but also from reduced pathologic personality features, reduced general psychopathologic dysfunction, and increased quality of life. In designing this randomized controlled trial, we decided to compare SFT and TFP because (1) these treatments seemed promising in an uncontrolled pilot study and therapists' individual clinical experiences (now further supported by open studies; Arntz, 1999; Clarkin, Foelsch, et al., 2001; Nordahl \& Nysaeter, 2005), (2) earlier studies already demonstrated specialized psychotherapeutic approaches are more effective than control conditions (including treatment-asusual and natural course; Bateman \& Fonagy, 1999; Lieb, Zanarini, et al., 2004; Linehan, Heard, et al., 1993; Perry, Banon, \& lanni, 1999; Verheul, van den Bosch, et al., 2003), and (3) no treatment-as-usual could control for treatment goals, intensity and session-frequency. 


\section{Methods}

\section{Study Design}

A multicenter, randomized 2-group design was used. Randomization to SFT or TFP was stratified across 4 community mental health centers and was performed by a study-independent person after the adaptive biased urn procedure (Schouten, 1995). We used this procedure (1) to keep allocation at each site unpredictable until the last patient to avoid unintentionally affecting ongoing screening procedures and (2) to keep the number of patients in balance between the conditions at each site. Each patient's first assessment occurred after inclusion and before randomization. Then, assessments were made every 3 months for 3 years by independent research assistants.

Therapists at secondary and tertiary community mental health institutes in the areas of the 4 participating treatment centers referred patients based on a clinical diagnosis of BPD. Patients were then assessed at each site (patients from The Hague were assessed in Leiden) using the Structured Clinical Interviews for the DSM-IV, Versions I and II (First, Gibbon, et al., 1997; First, Spitzer, et al., 1996; Groenestijn, Akkerhuis, et al., 1999; Weertman, Arntz, et al., 2000). Patients were further screened using a semistructured clinical interview, the Borderline Personality Disorder Severity Index, fourth version (BPDSI-IV; score range 0-90; Arntz, van den Hoorn, et al., 2003; Giesen-Bloo, Wachters, et al., 2006).

All the study therapists were affiliated with 1 of the 4 treatment centers. Nine experienced and extensively trained therapists with a master's degree in psychology diagnosed patients $(2$ therapists in Amsterdam, 4 in Leiden/The Hague, and 3 in Maastricht). All used interviews are highly reliable (McDermut \& Zimmerman, 2005; Weertman, Arntz, et al., 2003; Zimmerman, 1994). The BPDSI-IV cutoff score of 20 also discriminates patients with BPD from patients with other personality disorders (Giesen-Bloo, Wachters, et al., 2006), crosschecking the SCID-II BPD-diagnosis. Self-report questionnaires (the Dissociative Experiences Scale; Draijer \& Boon, 1993) and the Dutch Screening List for Attention Deficit Hyperactivity Disorder for adults (Kooij, 1997) were used in the screening process, if indicated, followed by the Semistructured Clinical Interview for DSM-IV - Dissociative Disorders (Boon \& Draijer, 1995; Steinberg, 1993) and/or an adjusted semistructured interview for Attention Deficit Hyperactivity Disorder (Dreessen, Stroux, \& Weckx, 1998). If low intelligence or illiteracy was suspected, the Wechsler Adult Intelligence Scale (Zimmerman, Woo, Glasser,et al., 1973) and/or the Dutch Adult Reading Test (Schmand, Lindeboom, \& van Harskamp, 1992) were administered. A positive screening procedure took 2 months, and this interval served as a patient's motivational check for undergoing intensive psychotherapy. Signed informed consent was obtained after full expla- 
nation of the procedures and of both therapies before the first assessment and randomization. Thirteen experienced and trained research assistants with higher vocational training in psychology assessed patients for treatment outcome measures (4 research assistants in Amsterdam, 5 in Leiden/The Hague, 4 in Maastricht). Study researchers, screeners, research assistants and SFT/TFP therapists were masked to treatment allocation during the screening procedure and the first assessment. The medical ethical committees of the participating centers approved the study. Participants did not receive compensation for screening or assessments but were exempt from the Dutch standard personal contribution to psychotherapy sessions (then $\$ 10$ per session). Participating in assessments was obligatory to receiving therapy.

\section{Patients}

Inclusion criteria were a main diagnosis of BPD, age 18 to 60 years, BPDSI-IV score greater than 20, and Dutch literacy. General exclusion criteria were psychotic disorders (except short, reactive psychotic episodes), bipolar disorder, dissociative identity disorder, antisocial personality disorder, attentiondeficit/hyperactivity disorder, addiction of such severity that clinical detoxification was indicated (after which entering treatment was possible), psychiatric disorders secondary to medical conditions, and mental retardation. These disorders were excluded since they generally need primary treatment. An exception is antisocial personality disorder because its "lie" feature is an explicit contraindication for TFP. Comorbid Axis-I and Axis-II disorders were allowed, as was medication use.

\section{Treatment Conditions and Therapists}

Both treatments were offered in 50-minutes sessions twice a week. Treatment protocols addressed the theoretical model, treatment frame, different phases, and the use of strategies and techniques (Clarkin, Yeomans, et al., 1999; Yeomans, Clarkin, et al., 2002; Young, 1999; Young, Klosko, et al., 2003). Central to SFT is the assumption of 4 schema modes specific to BPD. Schema modes are sets of schemas expressed in pervasive patterns of thinking, feeling and behaving. The distinguished modes in BPD are detached protector, punitive parent, abandoned/abused child, angry/impulsive child. In addition, some presence of the healthy adult is assumed. Change is achieved through a range of behavioral, cognitive and experiential techniques that focus on (1) the therapeutic relationship, (2) daily life outside therapy (also through home work assignments), and (3) past (traumatic) experiences. Recovery in SFT is achieved when dysfunctional schemas no longer control or rule the patient's life. Central to TFP 
is a negotiated treatment contract between patient and therapist, being the treatment frame. Change is achieved through analyzing and interpreting the transference relationship, focusing on the here-and-now context. Prominent techniques are exploration, confrontation and interpretation. Recovery in TFP is reached when good and bad representations of self (and of others) are integrated and when fixed primitive internalized object relations are resolved.

Nine therapists treated 1 patient each (4 SFT and 5 TFP), 28 treated 2 patients each (17 SFT and 11 TFP), and 7 treated 3 patients each ( 2 SFT and 5 TFP), with no between-group difference $(P=.27)$. Three therapists held doctoral degrees (1 SFT and 2 TFP), 37 held master's degrees (19 SFT and 18 TFP), and 4 held bachelor's degrees with postgraduate training (3 SFT and 1 TFP), with no betweengroup difference $(P=.42)$. All the therapists had previous therapy experience in their orientation with patients with BPD (mean [SD]: SFT, 9.95 [4.98] years; TFP, 11.73 [6.28] years), with no between-group differences $P=.39$ ). There were significantly more female SFT-therapists than TFP-therapists (15 vs $7 ; P=.04$ ), but without significantly contributing to treatment outcome $(P=.92)$.

Two supervisors initially trained the therapists. Essential to both treatments is supervision. Weekly local supervision with 4 to 5 SFT or TFP therapists, a 1 day central supervision every 4 months, and a 2-day central supervision every 9 months by Jeffrey Young, PhD (SFT), or Frank Yeomans, MD, PhD (TFP), were provided throughout the study. Psychiatrists from different orientations, including 2 SFT-therapists and 3 TFP-therapists, regularly evaluated the patients taking medication at the start and during treatment, prescribing according to good clinical practice, similar to American Psychiatric Association guidelines. No other concurrent treatments were allowed.

\section{Treatment Integrity Check}

Treatment integrity was monitored by means of supervision. Randomly selected audio tapes of each quarter and of sessions 1-6 (for the TFP-contract phase) were saved for evaluation. All the raters were independent of the study and masked to treatment outcome. One psychologist, masked to allocation, listened to 1 randomly selected tape of each patient, then stated the treatment administered. Eighty-five tapes were correctly classified; 1 SFT-tape was qualified TFP.

Thirty-three (partial) TFP contract phases were rated by trained graduate students in psychology using the Contract Rating Scale (Yeomans, Selzer, et al., 1992), covering patient and therapist responsibilities during and threats to treatment. Seven contract phases' tapes had extremely bad sound quality or were missing. Seventy-one ratings were analyzed, and the mean intraclass correlation coefficient (ICC) across 21 tapes was 0.46 (range 0.17-0.67). The con- 
tract setting adherence and competence had an average rating of 3.22 (range 2.86-3.54), a predetermined rating of 3 was considered adequate (Yeomans, Selzer, et al., 1993; Yeomans, Selzer, et al., 1992).

Other trained therapists for each orientation, assessed the TFP Rating of Adherence and Competence Scale (Clarkin, Yeomans, et al., 1999) or the SFT Therapy Adherence and Competence Scale for BPD (Young, Arntz, \& GiesenBloo, 1999). Both instruments consist of visual analog scale and Likert scale items and have an identical competence cutoff score of at least 60 . Fifty-six TFP tapes and 77 SFT tapes of the second and/or sixth trimester were rated (ICCs across 21 TFP and 20 SFT tapes that were rated twice).

Adherence to TFP was expressed in time percentage of TFP techniques, naming dyad-actors, and emergency focus. Only an average of $7.5 \%$ of the time (median, 4\%) was spent on non-TFP-techniques (ICC $=0.71)$. Valid actor naming occurred in 18 of 56 rated sessions $(\kappa=0.36)$, emergency focus was well kept $(\kappa=0.91)$. The median competence level for different aspects of interventions, treatment frame modification and emotional contact was 65.6 (ICC $=0.73$ ). The median global competence rating of the TFP therapists was 65 (ICC $=0.70$ ).

Adherence to SFT, as for overall appropriateness of used methods and techniques in SFT, was excellent (median, 90.00; ICC =0.76). No non-SFT techniques were observed. The median competence/quality level for applying SFT methods was $85.67(I C C=0.69)$, and the median global competence/quality SFT-therapist ratings was $73.00(I C C=0.78)$.

\section{Assessment}

The primary outcome measure was the score on the BPDSI-IV, a DMS-IV BPD criteria-based semistructured interview; this 70-item index represents the current severity and frequency of the DSM-IV BPD manifestations. The reference period is 3 months, which is appropriate in this study; and shows excellent psychometrics (Cronbach's $\alpha=.85$, interrater reliability, .99; validity and sensitivity to change; Arntz, van den Hoorn, et al., 2003; Giesen-Bloo, Wachters, et al., 2006). Previous research (Arntz, van den Hoorn, et al., 2003; Giesen-Bloo, Wachters, et al., 2006) found a cut-off score (Jacobson \& Truax, 1991) of 15 between patients with BPD and nonpatient controls, with a specificity of 0.97 and a sensitivity of 1.00 . The recovery criterion was, therefore, defined as achieving a BPDSI-IV score of less than 15 and maintaining this score untill the last assessment. A second criterion was reliable change (Jacobson \& Truax, 1991), which reflects individual clinically significant improvement. For the BPDSI-IV, reliable change was achieved when improvement was at least 11.70 points at the last assessment (Jacobson \& Truax, 1991). 
A secondary outcome measure was quality of life, assessed by means of 2 widely used and psychometrically sound self-report questionnaires: the EuroQolthermometer and the World Health Organisation Quality of Life Questionnaire (EuroQol Group, 1990; WHOQOL Group, 1998). The vertical EuroQolthermometer rating indicates one's experienced level between best (100) and worst (0) imaginable health status. The WHOQOL is a 100-item self-report questionnaire, and through the domains of physical health, psychological health, environment, personal convictions, social relationships and extent of independency, the WHO concept of quality of life is assessed. Other secondary outcome measures were assessed at 6-month instead of 3-month intervals and consisted of general psychopathologic measures and measures of SFT/TFP personality concepts, all in self-report format and with robust psychometric properties. More general measures included the BPD Checklist on the burden of BPD-specific symptoms (Arntz, van den Hoorn, et al., 2003; Giesen-Bloo, Arntz, et al., 2006), the Symptom Checklist-90 for subjective experience of general symptoms (Derogatis, Lipman, et al., 1973), the Rosenberg Self Esteem Scale (Rosenberg, 1965) and Miskimins Self-Goal(-Other) Discrepancy Scale for the difference between one's actual and desired/ideal self-perception (Miskimins, Wilson, et al., 1971). Theory specific instruments were the Young Schema Questionnaire on schemas underlying Young's theory (Rijkeboer, van den Bergh, et al., 2005; Schmidt, Joiner, et al., 1995), the Personality Disorder Belief Questionnaire-BPD section on BPD-specific beliefs derived from the Beck cognitive theory of personality disorders (Arntz, Dreessen, et al., 2004), the Inventory of Personality Organisation- borderline character pathology reflecting the facets of psychoanalytical borderline organizational structure developed after Kernberg's theory (Lenzenweger, Clarkin, et al., 2001) and the Defense Style Questionnaire (DSQ)-48 for mature, neurotic and immature defense mechanism use in daily life (Andrews, Pollock, et al., 1989; Giesen-Bloo, Arntz, et al., 2006). Principal component analysis of pretest secondary variables (Andrews, Pollock, et al., 1989; Derogatis, Lipman, et al., 1973; Giesen-Bloo, Wachters, et al., 2006) revealed 1 strong factor, on which only DSQ-mature defenses did not load (loading .15). Similar results were found when analyzing the linear trends of these variables: 1 strong factor and loading of 0.01 for DSQ-mature defenses. Highly similar results were obtained when other assessment points were analysed. After excluding DSQ-mature defenses, the pretest factor's eigenvalue was $7.51 \quad(57.8 \%$ variance) with factor loadings of 0.47-0.93 (median 0.78). The linear trend factor's eigenvalue was $8.63(66.4 \%)$ with factor loadings of 0.49-0.95 (median 0.82). Composite scores for pretest, last observation, and linear trends were derived by computing factor scores by using the regression method, and they are labeled psycho and personality pathology. 


\section{Sample size and data analysis}

BPDSI-IV-based power analyses indicated 45 patients per group are needed to detect a $22 \%$ versus $50 \%$ recovery difference between 2 groups by means of survival analysis, with a 2-sided significance level of $5 \%$ and a power of $80 \%$. An intention-to-treat approach was applied, using either the last observation during the 3-year treatment period or using last-observation-carried-forward method for trend analyses. First, treatment dropout survival analysis using Kaplan-Meier logistic regression (because of time dependency) was executed. Second, the effects of each treatment were evaluated using the McKean Schrader Test Statistic (MSTS; Wilcox, 2005) on the medians of pre-post changes. Then, Cox regression survival analyses on the BPDSI-IV recovery status and on the reliable change status for 3 years after the start of treatment, with treatment group as the covariate were executed. Time independency of relative risks (RRs) was checked. Between-group differences for outcome measures were examined using end point analyses and by analyzing the slopes of linear trend scores across all assessments during the 3 years because all linear trends on outcome measures differed significantly from zero in both conditions (Wilcoxon $z>1.97 ; P<.05$ ), except for DSQ-mature defenses (SFT: $P=.90$; TFP: $P=.50$ ). Therefore, outcome measures assessed every 3 (or 6 ) months were first transformed into linear trend scores, representing the linear change of these measures (except DSQmature defenses) during the 3-year study period.

Heteroscedasticity, skewness of distributions, regression outliers and leverage points analyses revealed that assumptions for parametric tests were not met. Robust analyses of covariance were, therefore, used, with pretest as the covariate, using Wilcox analysis of covariance (ANCOVA) on medians (Wilcox, 1997; 2005). All the tests were interpreted with a significance level of $5 \%$. Analyses were performed using SPSS, version 11.5 for Windows (survival analyses, within-group analyses, Chi-square tests) and the Rplus \& Rallfun Package, version 2.0.0 with extensions v1.v3 and v2.v3 (Wilcox ANCOVAs on medians).

\section{Results}

\section{Patient Accrual}

The study was conducted between September 1, 1999, and April 30, 2004. Patient flow is presented in Figure 1. Of 173 patients referred to the study centers, $40(23.1 \%)$ declined participation (12 patients after initial contact and 28 after having 1 or more appointments in the screening procedure). Another 45 patients (26.0\%) were not eligible for participation; 2 could not commit 
Figure 1. Patient flow of randomized controlled trial

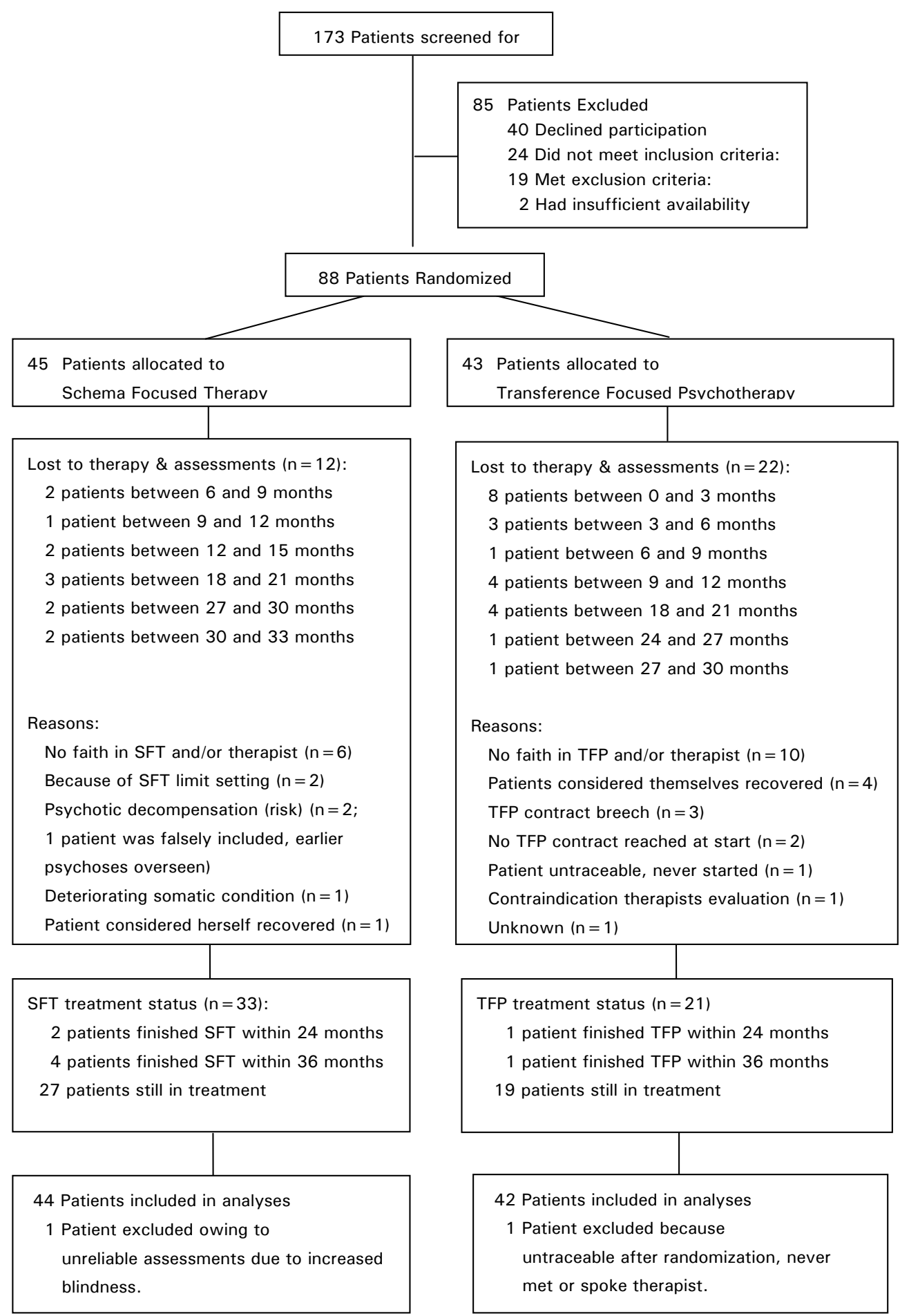


themselves to assessments every 3 months, 24 did not meet the inclusion criteria (14 no BPD diagnosis, 1 had an anorexia nervosa diagnosis that became lifethreatening during the screening procedure and required immediate, longer-term hospitalization, 1 was 17 years old, and 8 had BPDSI-IV scores <20). and 19 met the exclusion criteria $(6$ had bipolar disorder, 1 had psychotic disorder, 1 had valium addiction and refused detoxification, 2 had dissociative identity disorder, 7 had antisocial personality disorder, and 2 had attention-deficit/hyperactivity disorder). Eighty-eight patients $(50.9 \%$ of referrals) participated in the study.

Primary and secondary outcome variables and sociodemographic characteristics did not differ significantly among treatment centers. One SFT and 1 TFP-patient were excluded from analyses; the SFT-patient's poor eyesight made assessments unreliable, and the TFP-patient became untraceable after randomization.

Figure 2. Proportions of patients in TFP and SFT.

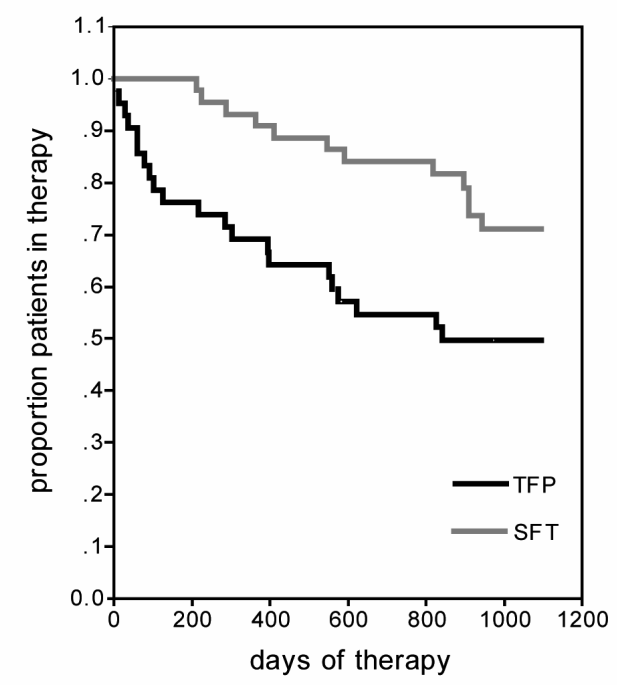

Six $(13.6 \%)$ of 44 SFT patients and 2 (4.8\%) of 42 TFP patients successfully terminated treatment within 3 years, only coded as such when both patient and therapist agreed on termination. No treatment was terminated because the therapist thought the patient was ready to end or owing to refusal of assessments. Twenty-seven SFT patients $(61.4 \%)$ and 19 TFP patients $(45.2 \%)$ were still in treatment after 3 years. Socalled completer SFT-patients (terminated treatment or still in treatment) had significant

fewer therapy sessions than completer TFP-patients (median: 189.5 vs 231.0; MSTS $=3.12 ; P=.002$ ). When patients dropped out of treatment can be read from Figure 1 and 2. No patient committed suicide. Survival analyses on the attrition rates show that TFP-patients have a significantly larger risk of dropout than SFT-patients (Kaplan-Meier method; log-rank statistic $=6.15, P=0.01$ ) (Figure 2). The SFT dropout patients had significantly more sessions than TFP dropout patients (median: 98 vs 34; MSTS = 3.53; $P<.001)$. 


\section{Treatment Groups at Baseline}

Table 1 gives an overview of patients' characteristics in both conditions at baseline. Age, sex, educational level, employment status, and psychotropic medication use did not differ significantly between treatment groups. Patients were mainly women in their 20s and 30s with average educational levels. The treatment groups had similar levels of BPD abnormality, quality of life and psycho- and personality pathology. Numbers of comorbid Axis-I and Axis-II disorders were equally distributed. A recent history of recent suicide planning, steps and/or attempts was significantly different between groups but had no effect on BPDSI-IV treatment outcome $(P=.22)$.

\section{Treatment Outcomes}

Results of the primary and secondary outcome measures are given in Table 2 and Figure 3. Significant effects at 3-years of SFT or TFP emerged for patients' reduction of BPDSI scores (SFT: MSTS $=-9.81, P<.001, d=2.96$; TFP: MSTS = 5.99, $P<.001, d=1.85$ ), improvement in quality of life (EuroQol thermometer score SFT: MSTS $=6.09, P=.001, d=1.84 ;$ TFP: $\mathrm{MSTS}=2.06, P=.044$, $d=0.64$; WHOQOL total score SFT: $\mathrm{MSTS}=4.86, P<.001, d=1.46$; TFP: MSTS $=3.73, P<.001, d=1.16)$ and reduction in psycho- and personality pathology (SFT: MSTS $=-6.73, P<.001, d=2.02$; TFP: $\mathrm{MSTS}=-2.75, P<.006$, $d=0.84)$. Both SFT and TFP-patients improved significantly on all DSM-IV BPD criteria ( $P<.001$ on all BPDSI-IV subscales) (Figure 4$)$. All effects were already apparent after 1 year.

Survival analysis on the BPDSI-IV recovery criterion with treatment group and baseline BPDSI-IV as predictors (covariates) showed a significant effect in favor of SFT (Wald =3.88, $P=.049, \mathrm{RR}=2.15$ with 95\% CI[1.00-4.59]), baseline BPDSI-IV was not significant $(P=.38)$ (Figure 4). Without baseline BPDSI-IV, the group effect was comparable (Wald $=4.04, P=.04, \mathrm{RR}=2.18$ with $95 \% \mathrm{Cl}[1.02-4.66]$ ). Differential dropout can only partly explain the difference between treatments because survival analysis with dropout status as an additional covariate was not significant for dropout, and the group effect became nonsignificant, although still in the same direction (group Wald $=2.67, P=.10, \mathrm{RR}=1.91$ with $95 \% \mathrm{Cl}[0.88-4.14]$; dropout Wald $=1.90, P=.17, \mathrm{RR}=1.84$ with $95 \% \mathrm{Cl}[0.77-4.35])$. The group-effect persisted when the analysis was adjusted for the use of psychotropic medication as time-dependent covariate (13 assessments; Wald $=4.42, P=.04, \mathrm{RR}=2.26$ with 95\% CI[1.06-4.85]). Baseline BPDSI-IV was not significant $(P=.33)$. Psychotropic medication use had a significant negative effect on recovery (Wald $=6.21, P=.013$, $\mathrm{RR}=0.38$ with $95 \% \mathrm{CI}[0.18-0.81]), 55 \%$ of patients who did not use 
Table 1. Sociodemographic and clinical characteristics of 86 study participants

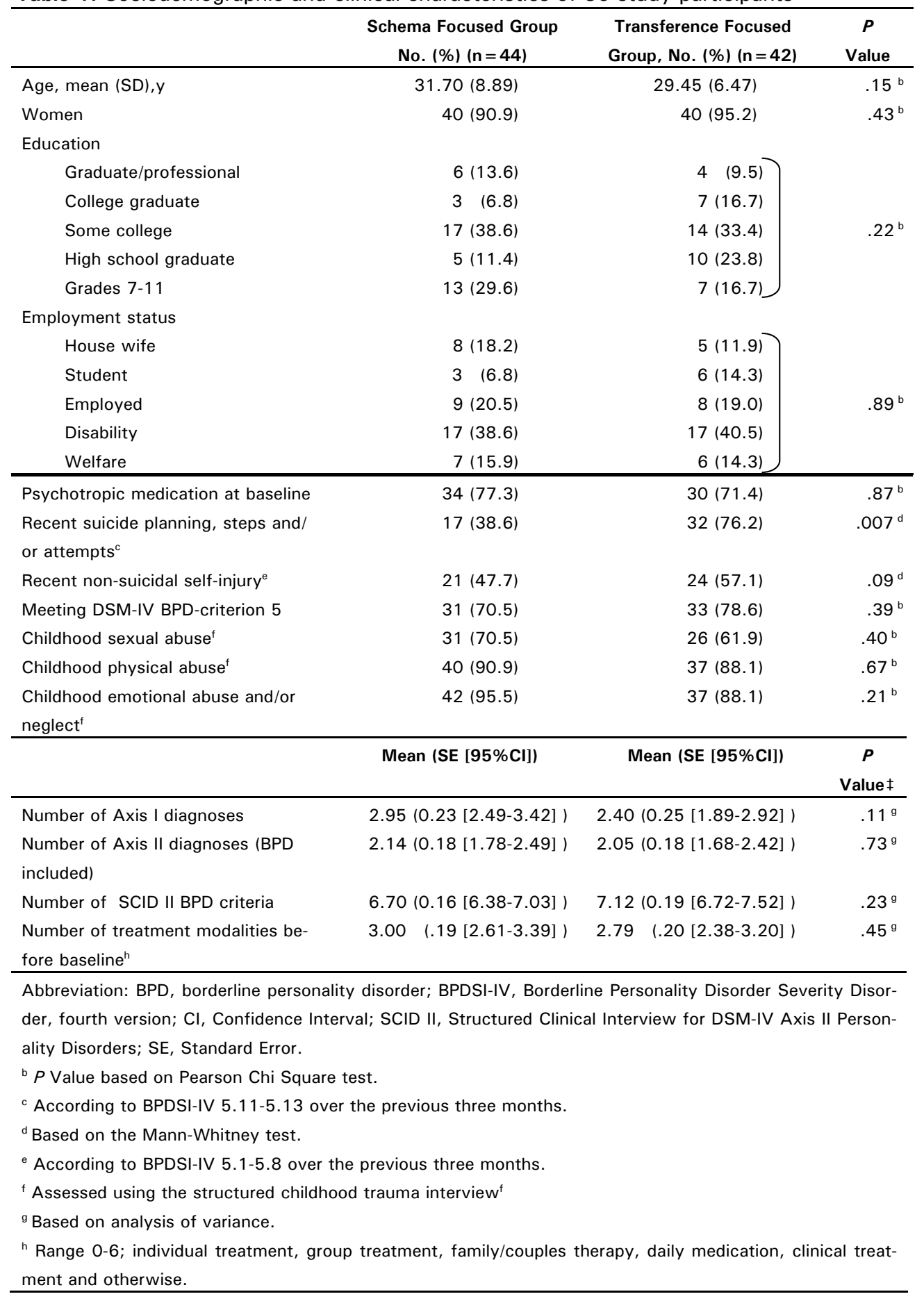


Table 2. Primary and Secondary Outcome Measures in 86 study participants ${ }^{\text {aa }}$

\begin{tabular}{|c|c|c|c|}
\hline & $\begin{array}{l}\text { Schema Focused Group } \\
\text { No. }(\%)(N=44)\end{array}$ & $\begin{array}{c}\text { Transference Focused Group } \\
\text { No. (\%) }(\mathrm{N}=42)\end{array}$ & $\begin{array}{c}P \\
\text { Value }\end{array}$ \\
\hline Recovery criterion 15 , yes & $20(45.5)$ & $10(23.8)$ & $.04^{\mathrm{a}}$ \\
\hline \multirow[t]{2}{*}{ Reliable Change, yes } & $29(65.9)$ & $18(42.9)$ & $.03^{a}$ \\
\hline & Median (SE [95\%CI]) & Median (SE [95\%CI]) & $\begin{array}{c}P \\
\text { Value }\end{array}$ \\
\hline \multicolumn{4}{|l|}{ 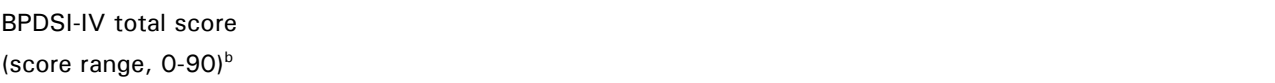 } \\
\hline Baseline & $33.53(1.23$ [31.12-35.94]) & $34.37(1.23[31.96-36.78])]$ & \\
\hline 12-mo treatment & $22.18(1.67[18.91-25.45])$ & $25.13(1.76[21.68-28.58])$ & $.01^{\mathrm{c}, \mathrm{d}}$ \\
\hline 24-mo treatment & $17.77(1.21[12.32-20.14])$ & $23.38(1.79$ [19.87-26.89] ) & \\
\hline 36-mo treatment & $16.24(1.51[13.28-19.20])$ & $21.87(1.71[17.95-25.79])$ & $.005^{\mathrm{c}, \mathrm{e}}$ \\
\hline \multicolumn{3}{|l|}{ EuroQol Thermometer } & score (range $0-100)^{f}$ \\
\hline Baseline & $(3.29[43.55-56.45])$ & $(2.72[49.67-60.33])$ & \\
\hline 12-mo treatment & $56 \quad(2.52[51.06-60.94])$ & $64 \quad(4.85[54.49-73.51])$ & $<.001^{\mathrm{c}, \mathrm{d}}$ \\
\hline 24-mo treatment & $(3.49[58.16-71.84])$ & $(4.85[59.49-78.51])$ & \\
\hline 36-mo treatment & $64.5(4.66[55.37-73.63])$ & $67.5(2.91[61.80-73.20])$ & $.70^{\mathrm{c}, \mathrm{e}}$ \\
\hline \multicolumn{4}{|l|}{$\begin{array}{l}\text { WHOQOL total scores } \\
\text { (range } 4-20)^{f}\end{array}$} \\
\hline Baseline & $10.33(0.19[9.96-10.70])$ & $10.42(0.09[10.24-10.60])]$ & \\
\hline 12-mo treatment & $11.17(0.26[10.66-11.68])$ & $11.17(0.19[10.80-11.54])$ & $.03^{c, d}$ \\
\hline 24-mo treatment & $11.42(0.36[10.71-12.13])$ & $11.23(0.26[10.72-11.74])$ & \\
\hline 36-mo treatment & $11.59(0.29[11.02-12.16])$ & $11.09(0.19[10.72-11.46])$ & $.16^{\mathrm{c}, \mathrm{e}}$ \\
\hline \multicolumn{4}{|l|}{$\begin{array}{l}\text { Psycho- \& Personality } \\
\text { factor score }\end{array}$} \\
\hline Baseline & $0.36 \quad(0.06[0.24-0.48])$ & $0.64(0.13[0.15-0.89])]$ & \\
\hline 12-mo treatment & $-0.14(0.18[-0.49-0.21])$ & $0.22(0.13[-0.03-0.47])$ & $<.001^{\mathrm{c}, \mathrm{d}}$ \\
\hline 24-mo treatment & $-0.39(0.16[-0.70--0.08])$ & $-0.02(0.15[-0.31-0.27])$ & \\
\hline 36-mo treatment & $-0.56(0.12[-0.80--0.32])$ & $0.13(0.18[-0.22-0.48])$ & $.007^{\mathrm{c}, \mathrm{e}}$ \\
\hline \multicolumn{4}{|c|}{$\begin{array}{l}\text { Abbreviation: ANCOVA, analysis of covariance; BPDSI-IV, Borderline Personality Disorder Severity Index, } \\
\text { fourth version; CI, Confidence interval; SE, Standard Error; WHOQOL, World Health Organization Quality of } \\
\text { Life assessment. }\end{array}$} \\
\hline \multicolumn{4}{|c|}{${ }^{b}$ Higher scores indicate more severe borderline personality disorder abnormalities. } \\
\hline \multicolumn{4}{|c|}{$\begin{array}{l}{ }^{c} \text { Based on Wilcox ANCOVA: robust ANCOVA }{ }^{41,42} \text { based on Wilcox Rallfun package (Rand R. Wilcox, De- } \\
\text { partment of Psychology, University of Southern California, Los Angeles; www-rfc.usc.edu/ rwilcox/). } \\
\text { d Linear Trend Wilcox ANCOVA on medians across } 13 \text { assessments (psycho-and personality } 7 \text { assessments). } \\
\text { e Endpoint Wilcox ANCOVA on medians. }\end{array}$} \\
\hline \multicolumn{4}{|c|}{ Higher scores indicate higher levels of quality of life } \\
\hline${ }^{g}$ Higher scores indicate mor & ho- and personality pathology & & \\
\hline
\end{tabular}


medication at start recovered compared with $28 \%$ of those using medication. The treatment group $X$ medication interaction was not significant. Patient use of psychotropic medication across time is shown in Figure 5. In addition, 1 TFP patient started taking a mood stabilizer 3 months after the start of treatment and continued throughout the study.
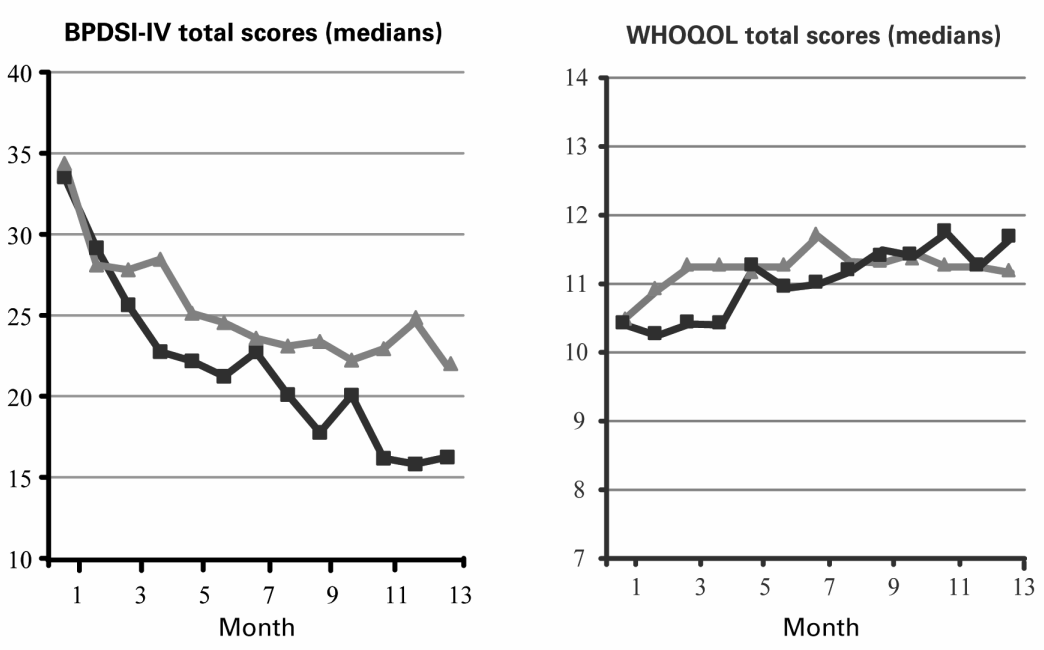

EuroQol thermometer score (medians)

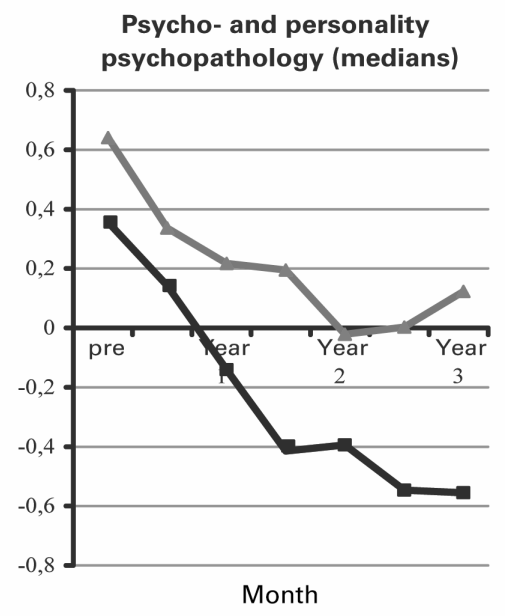

Figure 3. Median primary and secondary outcome measure scores.

Note BPDSI-IV total scores, range 0-90; WHOQOL total scores, range 4-20; Euroqol thermometer scores, range 0-100; Composite score psycho- and personality pathology factor scores. 
Survival analysis on reliable change status and baseline BPDSI-IV again showed a SFT effect (Figure 4) (Wald $=6.90, P=0.009, R R=2.33$ with $95 \%$ CI[1.24-4.37]). As expected for the BPDSI-IV based reliable change criterion, baseline BPDSI-IV had a significant effect (Wald $=15.01, P<.001, R R=1.07$, CI[1.03-1.10]). The SFT effect remained after including time-dependent psychotropic medication use (SFT Wald $=7.40, P=.007, \mathrm{RR}=2.38$ with $95 \% \mathrm{CI} 1.27$ 4.43]; medication Wald $=8.54, P=.003, \mathrm{RR}=.40, \mathrm{Cl}[0.22-0.74])$. Time $\mathrm{X} \mathrm{RR}$ interactions were not significant (recovery $P=.13$, reliable change $P=.20$ ).
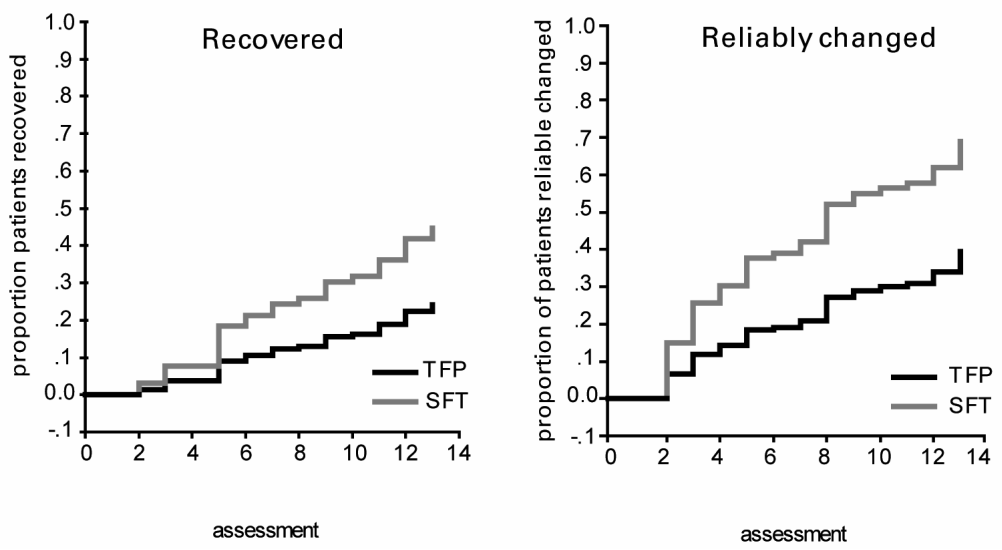

Figure 4. Proportion of patients recovered and reliable changed for TFP and SFT, adjusted for baseline BPDSI-IV.

Results of the Wilcox ANCOVA on BPDSI-IV medians of the last observation again proved that SFT is more effective than TFP (MSTS $=2.83, P<0.005$, $d=0.62$ ). Subsequent linear trend analysis using Wilcox ANCOVA on the BPDSIIV of all 13 assessments demonstrated a similar group effect in favor of SFT (MSTS $=2.66, P=.01, d=0.58$ ). Wilcox robust ANCOVA tests at the last observation of all median BPDSI-IV subscales revealed that the SFT group improved significantly more than the TFP group with respect to abandonment fears $(P=.04)$, relationships $(P=.03)$, identity disturbance $(P=.02)$, impulsivity $(P=.03)$, (para)suicidal behavior $(P=.048)$, and dissociative and paranoid ideation $(P=.02)$ (Figure 6$)$. No significant group differences were found for other subscales, although anger showed a trend in favor of SFT $(P=.06)$.

The Wilcox ANCOVA on EuroQol thermometer 3-years treatment medians did not show a group effect. However, the linear trend analysis using Wilcox ANCOVA on the EuroQol thermometer medians across 3 years revealed a significantly sharper increase in ratings for the SFT group than for the TFP group 
(MSTS $=2.16, P=0.03, d=0.46$ ). A small crossing effect was observed on the WHOQOL: SFT-patients had slightly lower total scores than TFP-patients at baseline and slightly higher total scores after 3 years of treatment. No statistically significant group effect emerged when the last observation medians were compared using Wilcox ANCOVA, and SFT had a stronger increase than TFP when the linear trend across all WHOOOL assessments was analysed using Wilcox ANCOVA $(P<0.001)$.

Figure 5. Patients using psychotropic medications by condition.

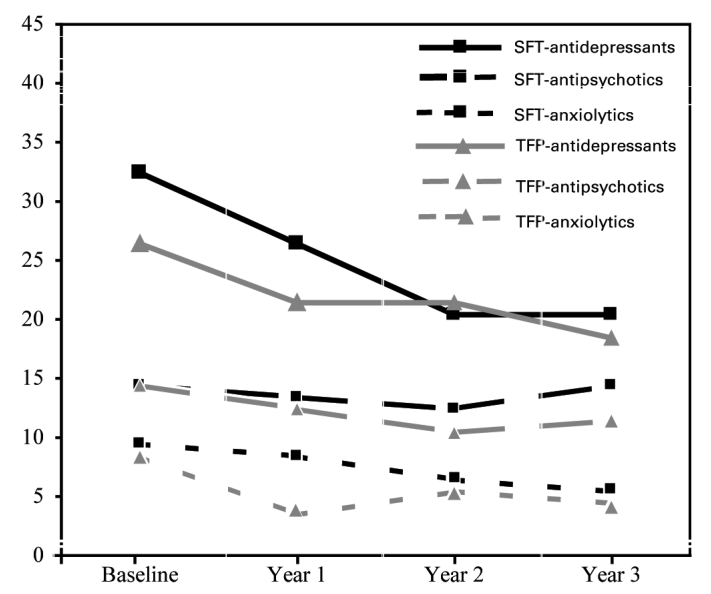

Wilcox ANCOVA on psychoand personality pathology factor scores of last observation medians showed a significantly larger effect for SFT than for TFP (MSTS $=2.68, P=.007, d=0.58$ ). Linear trend analysis on the psycho- and personality pathology factor scores across 3 years using Wilcox ANCOVA showed a significantly steeper decline for the SFT group than for the TFP group (MSTS $=3.30, P<.001, d=0.72$ ).

\section{Discussion}

Three years of SFT and TFP sessions proved to bring about a significant change in patients' personality, shown by reductions in all BPD symptoms and general psychopathologic dysfunction, increases in quality of life, and changes in associated personality features. Using intention-to-treat analysis with adjustments for baseline assessments, SFT and TFP effectiveness became apparent at 12 months of treatment and was further extended at 3 years of treatment. Schema-focused therapy was superior to TFP with respect to reduction in BPD manifestations, general psychopathologic dysfunction, and change in SFT/TFP personality concepts. All in all, it seems that changes in manifest (BPD) psychopathologic dysfunction go hand in hand with changes in pathologic personality features. An explanation may be that both treatments address the level of personality, not merely the 'surface' symptom level. Schema-focused therapy was not consistently dominant over TFP with respect to patients' improved quality of life, as trend and end-point analyses yielded different results. 

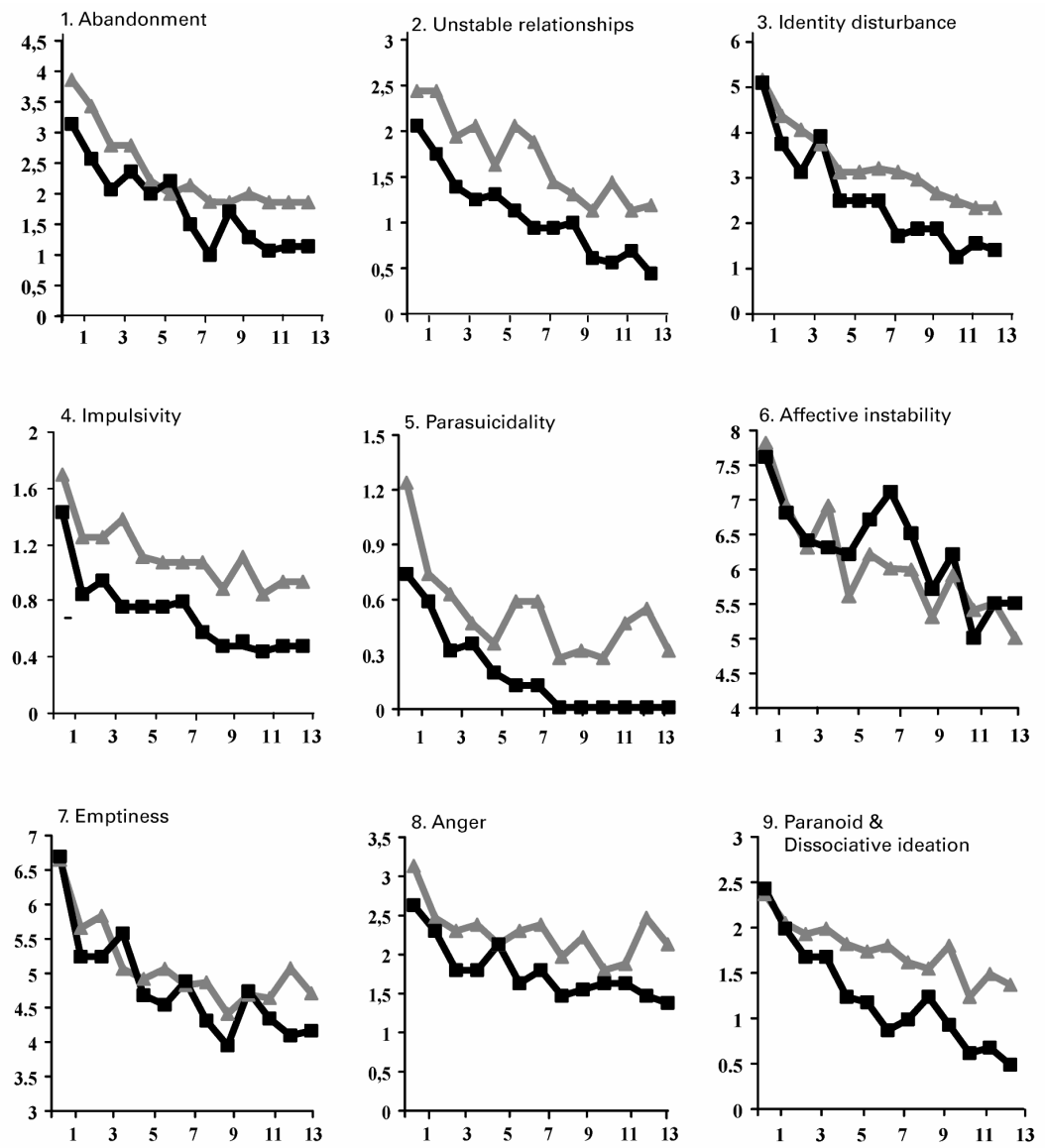

Figure 6. Median Borderline Personality Disorder Severity Index, fourth version (BPDSI-IV).

Note BPD manifestations according to the DSM-IV criteria as assessed with the BPDSI-IV for every assessment. SFT ( $\mathbf{\square} ; \mathrm{n}=44)$ and $\operatorname{TFP}(\Delta ; \mathrm{n}=42)$.

Schema-focused therapy had a significantly lower attrition rate than TFP. However, both treatments demonstrate that patients with BPD can be motivated for and continue prolonged outpatient treatment. To our knowledge, this is the first 3-year controlled treatment effectiveness study for BPD. An additional 1year follow-up period after the initial 3-year treatment period has recently been completed. The cost-effectiveness of SFT and TFP will then be determined.

Caution is recommended when comparing the current findings with study results on outpatient dialectical behavior therapy (DBT) and psychoanalytically oriented mentalization based treatment (MBT; Linehan, Armstrong, et al., 1991; 
Bateman \& Fonagy, 1999). Most essential is a different primary aim in DBT and MBT, namely, to reduce the self-destructive psychopathologic dysfunction of BPD and not its overall personality change. Comparisons are further hampered by differences in treatment setting (outpatient versus partial hospitalization in MBT), time investment/intensity for the patient (e.g. $>4$ hours weekly in MBT, at least $3-3 \frac{1}{2}$ in DBT, and 2 in SFT and TFP), number of therapists involved (MBT > DBT > SFT/TFP), use of (severity) outcome measures, and studied treatment duration ( 1 year for DBT, $1 \frac{1}{2}$ years MBT, and 3 years SFT/TFP). Still, it remains that the present study established effectiveness for all aspects of BPD pathology and, moreover, quality of life with large treatment effect sizes. Regarding attrition rates and reduction of (para)suicidality (BPDSI-IV subscales impulsivity and parasuicidality), SFT holds up well compared to other BPD treatments studies (Bateman \& Fonagy, 1999; Linehan, Armstrong, et al., 1991). It can be argued that DBT and MBT are possibly most optimal for a subgroup of patients with BPD who have prominent parasuicidal abnormalities, whereas SFT and TFP are meaningful for the wide range of patients with BPD. The 1-year attrition rate of the present TFP group seems to be similar to that in an uncontrolled TFP-study by Clarkin, Foelsch, et al. (2001) (the difference was not significant; $\left.\chi^{2}(\mathrm{df}=1, \mathrm{n}=60)=0.33, P=0.57\right)$, although comparing is problematic because patients in the uncontrolled TFP study knew beforehand what therapy they would receive and that the free study treatment period was limited to 1 year. Regarding (para)suicidal behavior (BPDSI-IV subscale), our TFP-patients' improvement seems to be larger than the uncontrolled TFP study (1-year $d^{\prime}$ s: TFP Clarkin et al. 0.15-0.46; present study TFP 0.67). Compared with 1 year of cognitive therapy (Brown, Newman, et al., 2004), our data indicate that SFT and TFP seem to yield better results with respect to a study's main outcome measures (1year $d$ 's: SFT 0.43-1.03; TFP 0.09-0.99; CT 0.22-0.55) (Brown, Newman, et al., 2004; Clarkin, Foelsch, et al., 2001). A single case series of 18 to 36 months of SFT with large effect sizes (1.8-2.9) further support the potential of SFT in treating BPD (Nordahl \& Nysaeter, 2005). Psychotropic medication use was related to poorer outcome (but unrelated to BPD severity at baseline). Whether more difficult-to-treat patients are generally taking medication, whether medication counteracts psychotherapy (Simpson, Yen, et al., 2004), or whether other factors are involved remains unclear.

Despite that 30 patients had reached the BPDSI-IV recovery criterion, many of them were still in treatment after 3 years. First, patient and therapist were masked to assessment results to avoid unintentionally affecting study participants. Second, changing BPD symptoms is one thing, but installing safe attachment, functional conscience, and functional and positive self- and other views is another thing. For example, self-mutilation or relation crises may have stopped, but this does not mean that a patient's self-esteem has risen. 
A limitation of this study is that most research assistants learned their patients' treatment allocation as the study progressed, as patients talked about their treatment and therapists. However, results of secondary computerassessed self-report measures (in an individual, private setting), concurred with the observer-rated (interview) findings, making it unlikely that results can be contributed to knowledge of treatment allocation. In addition, study psychiatrists were not per se masked to treatment allocation. A third limitation is the absence of a natural-course control group.

Recently, Zanarini and colleagues (Zanarini, Frankenburg, Hennen, et al., 2003) found that symptomatic improvement of BPD phenomenology is common and stable among BPD-patients during a 6-year natural-course follow-up. A difficulty in interpreting the findings of Zanarini and colleagues is whether improvement is the natural course in BPD or the result of received treatments and/or other factors. Note that previous studies found specialized psychotherapy to be superior to natural-course or control treatments (Bateman \& Fonagy, 1999; Lieb, Zanarini, et al., 2004; Linehan, Armstrong, et al., 1991; Perry, Banon, et al., 1999; Verheul, van den Bosch, et al., 2003). Moreover, the differences in outcome between SFT and TFP are due to treatment, otherwise results should have been the same after three years of treatment.

In conclusion, this study contributes to a positive treatment perspective for BPD by lending support to SFT, as a valid evidence-based practice. However, straightforward recommendations for clinical practice cannot and should not be made on the basis of only 1 effectiveness study. More research is needed to replicate and subsequently solidify current findings, for example, comparisons of SFT/TFP with other specific BPD treatments, treatment-as-usual, and the natural course. Furthermore, possible adjustments within the treatment frames could be explored, as health care efficiency is the target of many countries' policies and economics. Hypothesized effective ingredients of SFT for patients with BPD may be (1) the model's transparency, (2) the therapist's "reparenting" attitude on the attachment issues of patients with BPD, (3) the many hands-on techniques/ strategies that offer a patient structure and control, and (4) the opportunity to contact the SFT-therapist (within limits) between sessions. Future research needs to identify factors that facilitate optimal treatment indication.

\section{Acknowledgements}

We wish to acknowledge the contribution of the participating BPD patients, the SFT and TFP therapists; consultants Jeffrey Young PhD (SFT) and Frank Yeomans MD PhD (TFP), the screening-psychologists and research assistants. Furthermore we sincerely acknowledge the statistical advice of Hubert Schouten PhD, Erik Schouten, and Rand Wilcox PhD. 


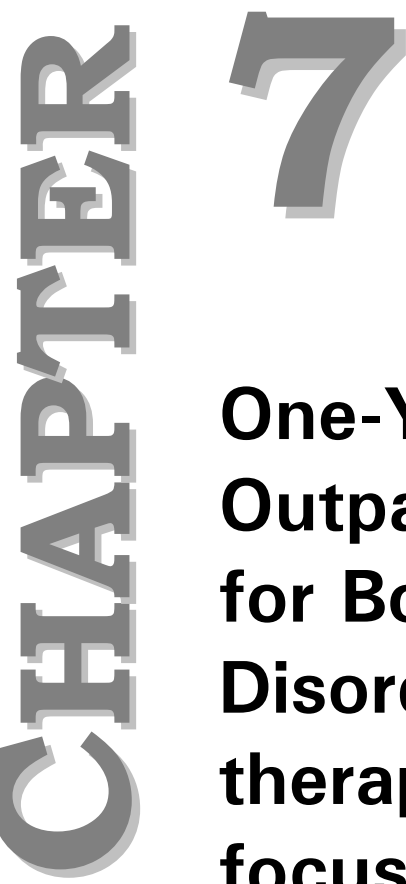

One-Year follow-up of Outpatient Psychotherapy for Borderline Personality Disorder: Schema focused therapy versus Transference focused psychotherapy

Giesen-Bloo, J., van Dyck, R., Spinhoven, P., van Tilburg, W., Dirksen, C., van Asselt, T., Kremers, I., Nadort, M., Arntz, A. (Submitted for publication) 


\section{Abstract}

Objective Borderline Personality Disorder is a severe and chronic psychiatric condition, prevalent throughout health care settings. Few treatments are evidence based, little is known of prolonged treatments' effects and maintenance. The present study examined the maintenance of the effectiveness and relative effectiveness of three years of schema focused therapy (SFT) or transference focused therapy (TFP) for borderline patients after a 1-year follow-up period. The study also assessed whether medication use, treatment dropout, study dropout and treatment status (still in treatment or treatment completed) influenced the condition effects.

Method One-year naturalistic follow-up of a randomized controlled two group design in which eighty-six patients with borderline personality disorder of four general community mental health centers participated. Main Outcome Measures were the Borderline Personality Disorder Severity Index, 4th version (BPDSI-IV), quality of life, general psychopathology and measures of SFT/TFP personality concepts. As assumptions for parametric tests were not met, robust analyses of covariance were employed with pretest as covariate, using Wilcox ANCOVA on medians.

Results Data of 44 SFT-patients and 42 TFP-patients were analyzed. Using an intention-to-treat approach, statistically and clinically significant improvements in both treatment groups endured on almost all measures. Differences in improvement between SFT and TFP remained, but additional improvement achieved during the follow-up year was not significant. Recovery rates were $52.3 \%$ (SFT) and $28.6 \%$ (TFP).

Conclusions Treatment effects after 3-years of SFT/TFP further increased at 1-year follow up, and SFT was superior to TFP on all measures except fot equal effects on quality of life. The differences between SFT and TFP on the primary outcome measure did not depend on medication use, dropout status or completion of treatment. The study contributes to a positive treatment perspective for BPD. 


\section{Introduction}

Borderline personality disorder (BPD) has for long been viewed as a serious, chronic and difficult to treat psychiatric condition with considerable impact on the personal and societal level. However, recent years demonstrated considerable progress in the development of manualized treatment possibilities, specific for BPD (Bateman \& Fonagy, 2004; Layden, Newman, et al., 1993; Linehan, 1993a; b; Marziali \& Munroe-Blum, 1994; Meares, 2004; Pretzer, 1990; Ryle, 1997; Yeomans, Clarkin, et al., 2002; Young, Klosko, et al., 2003). Unfortunately most treatments are examined in uncontrolled studies, only few are supported by randomized controlled trials (Bateman \& Fonagy, 1999; Giesen-Bloo, van Dyck, et al, 2006; Linehan, Armstrong, et al, 1991; Munroe-Blum \& Marziali, 1995; Verheul, van den Bosch, et al, 2003). Efficacy is often assessed and manifested by good treatment retention, and reduced suicidal behavior, impulsivity and hospitalizations. Even though the effects found are somewhat limited, i.e. not covering the full range of BPD manifestations, the longer-term follow-up reports available for these treatments (Bateman \& Fonagy, 2001; Linehan, Heard, et al, 1993; Stevenson \& Meares, 2005) read positively in that effects are maintained over time. The long-term perspective for BPD patients offered by more general, both retrospective and prospective, follow-up studies ranges from pessimistic to moderately optimistic (McGlashan, 1986; Links, Heslegrave \& van Reekum, 1998; Paris, Brown \& Nowlis, 1987; Sandell, Alfredsson, Berg, et al, 1993; Stone, 1993; Zanarini, Frankenburg, et al, 2003).

This paper describes the 1-year naturalistic follow-up results of a multicenter randomized controlled trial, on which we recently reported (Giesen-Bloo, van Dyck, et al, 2006). In this RCT, we compared two prolonged outpatient treatments that address patients' levels of personality (not merely symptom level) and aim for full recovery from BPD pathology: Schema Focused Therapy (SFT; Young, 1994; Young, Klosko, et al., 2003) and Transference Focused Psychotherapy (TFP; Clarkin, Yeomans, et al, 1999; Yeomans, Clarkin, et al., 2002). Three years of biweekly SFT and TFP sessions resulted in significant structural change in patient's personality, shown by reduction of all BPD symptoms and general psychopathology, increase in quality of life and change in associated personality features. Effectiveness became apparent early on in treatment. SFT was significantly superior to TFP with respect to reduction in BPD pathology, general psychopathology, and change in measures of SFT/TFP personality concepts. SFT was superior to TFP in reduction of manifestations of six of the nine DSM-IV BPD criteria. Furthermore, SFT had a significantly lower attrition rate than TFP.

Aim of the present study was to investigate whether (differential) treatment effects found were maintained or changed, at 1 year follow-up. A secondary aim 
was to assess whether or not differences between SFT and TFP depend on medication use, treatment dropout, study dropout, and treatment completion status.

\section{Methods}

The study design, screening and randomization procedures, patient characteristics, health care settings, treatment conditions, therapists and details of outcome variables are described in the original article (Giesen-Bloo, van Dyck, et al., 2006). The naturalistic follow-up study period was between September 2003 and April 2005.

\section{Subjects}

Eighty-six BPD-patients (44 SFT, 42 TFP), analyzed in the original RT, were approached for naturalistic follow-up assessment one year after the experimental 3-year treatment period. The two treatment groups did not significantly differ on demographic or clinical characteristics at baseline, except for recent suicidal manifestations, which did not influence the results however. After the experimental 3-year randomly assigned manual-based treatment period, it was open to the therapist how and/or whether treatment was to be continued. All ongoing SFT/TFP treatments were continued or terminated according to regular procedures.

\section{Assessment}

Outcome variables. The primary outcome measure was the BPDSI-IV, a DSM-IV BPD criteria based semi-structured interview; the 70-item index represents the current severity and frequency of DSM-IV BPD manifestations. The reference period is three months. The total score has excellent psychometric properties (Cronbach's $\alpha 0.85$, interrater reliability 0.99 , validity and sensitivity to change) (Arntz, van den Hoorn, et al, 2003; Giesen-Bloo, Wachters, et al, 2006). Previous research (Arntz, van den Hoorn, et al, 2003; Giesen-Bloo, Wachters, et al, 2006) found a cut-off score (Jacobson \& Truax., 1991) of 15 between BPD patients and non-patient controls, with a specificity of 0.97 and a sensitivity of 1 . The recovery criterion was therefore defined as achieving a BPDSI-IV score of less than 15, and maintaining this score till the last assessment. A second criterion was reliable change (Jacobson \& Truax., 1991), which 
reflects individual clinical significant improvement. For the BPDSI-IV, reliable change was achieved when improvement was at least 11.70 points at the last assessment.

A secondary outcome measure was quality of life, assessed with two widely used and psychometrically sound self-report questionnaires: the EuroQolthermometer and the World Health Organisation Quality of Life Questionnaire (WHOQOL; the EuroQol Group, 1990; the WHOQOL Group, 1998). The vertical Euroqol-thermometer rating indicates one's experienced level between best (100) and worst $(0)$ imaginable health status. The WHOQOL is a 100 -item self-report questionnaire, and through the domains of physical health, psychological health, environment, personal convictions, social relationships and extent of independency, the WHO concept of quality of life is assessed. Other secondary outcome measures were assessed at 6-month instead of 3-month intervals and consisted of general psychopathology measures and measures of SFT/TFP personality concepts, all in self-report format and with robust psychometric properties. More general measures included the BPD Checklist on the burden of BPD-specific symptoms (Giesen-Bloo, Arntz, et al, 2006), the Symptom Checklist-90 for subjective experience of general symptoms (Derogatis, Lipman, et al, 1973), the Rosenberg Self Esteem Scale on self-esteem (Rosenberg, 1965) and Miskimin's Self-Goal(-Other) Discrepancy List on the difference between one's actual and desired/ideal self-perception (Miskimins, Wilson, et al, 1971). Theory specific instruments were the Young Schema Questionnaire on schema-sets underlying Young's theory (Rijkeboer, van den Bergh, et al, 2005), the Personality Disorder Belief Questionnaire-BPD section on BPD-specific beliefs derived from Beck's cognitive theory of personality disorders (Arntz, Dreessen, et al, 2004), the Inventory of Personality Organisation- borderline character pathology reflecting the facets of psychoanalytical borderline organizational structure developed after Kernberg's theory (Lenzenweger, Clarkin, et al, 2001) and the Defense Style Questionnaire (DSQ)-48 on mature, neurotic and immature defense mechanism use in daily life (Andrews, Pollock, et al, 1989; Giesen-Bloo, Arntz, et al, 2006). Principal component analysis of pre-test secondary variables (Wilcox, 1997; 2005; 2006) revealed one strong factor, on which only DSQ-mature defenses did not load (loading .15). Highly similar results were obtained when the followup assessment was analysed. After excluding DSQ-mature defenses, the pre-test factor's Eigenvalue was 7.51 (57.75\% variance) with .47-.93 factor loadings (median .78). Composite scores for pre-test, last observation and follow-up, were derived by computing factor scores with the regression method, and are labelled "psycho-and personality pathology". 


\section{Data analysis}

Conservative intention-to-treat analyses with last-observation-carriedforward for missing follow-up values were applied $(N=86)$. Mixed effect modeling analyses were not indicated, as drop-out status was associated with both condition and poorer treatment response, so that the randomness assumption was violated. For the primary outcome measure, we investigated whether the data of the patients who were assessed at follow-up lead to different conclusions compared to the intention-to-treat analysis. We also assessed treatment differences in those who were still in treatment, and in those that had regularly finished treatment.

Heteroscedasticity, skewness of distributions, regression outliers and leverage points analyses revealed that assumptions for parametric tests were not met. Therefore, robust analyses of covariance were employed with pretest as covariate, using Wilcox ANCOVA on medians (Wilcox, 1997; 2005).

Effects of each treatment were evaluated with the McKean Schrader Test Statistic (MSTS Wilcox, 2005) on the medians of baseline to follow-up changes, and on the medians of post to follow-up changes. Then, logistic regression analyses on the BPDSI-IV recovery status and on the reliable change status at follow-up with treatment group and baseline BPDSI-IV as covariates, were executed. Between-group differences were examined with end point (i.c. follow-up) analyses. Because of the small N's ANCOVA's within therapy regularly finished and still-in-therapy subgroups were done with a bootstrap method. This robust ANCOVA yields a between group $P$-level after conditioning on the covariate (Wilcox, 2006).

All tests were interpreted with a significance level of $5 \%$. Analyses were performed using the Statistical Package for Social Sciences, version 12.0 for Windows (within-group analyses, Chi-square tests) and the Rplus \& Rallfun Package, version 2.0.0 with extensions v1.v3 and v2.v3 (Wilcox ANCOVA's on medians).

\section{Results}

\section{Subjects}

Twenty-six SFT- and 19 TFP-patients were still in treatment after the experimental treatment period of 3 years. Treatment intensity (Table 1) for SFTpatients was significantly less than for TFP-patients during the follow-up year (Mann whitney $U=112, Z=-3.21, P=.001$ ), $P=.004$ ). With respect to treatment completers, meaning those patients that are still in treatment after four 
years or successfully terminated treatment, median number of treatment sessions for SFT is 222, for TFP 292. These are also significantly different from each other (MSTS $=4.45, P<.001, d=1.22$ ). There were no SFT or TFP treatment dropout patients during the follow-up year. In addition, the reduced use of psychotropic medication in both groups was maintained. Actual follow-up data could be collected for 22 SFT- and 21 TFP-patients. If indicated, status information of other patients who completed the original 3-year study period was obtained of the patient's therapist.

Table 1. Treatment status and treatment frequency of patients still in treatment during the follow-up year.

\begin{tabular}{|c|c|c|c|}
\hline Still in treatment & $\begin{array}{c}\text { Schema Focused } \\
\qquad \begin{array}{c}\text { No. (\%) } \\
N=26\end{array}\end{array}$ & $\begin{array}{c}\text { Transference Focused } \\
\qquad \begin{array}{c}\text { No. (\%) } \\
N=19\end{array}\end{array}$ & $\begin{array}{c}P \\
\text { Value } \dagger\end{array}$ \\
\hline \multicolumn{4}{|l|}{$\begin{array}{l}\text { Treatment frequency during } \\
\text { follow-up year }\end{array}$} \\
\hline Twice weekly & $2 \quad(7.7)$ & $10(52.6)$ & \\
\hline once weekly & $8(30.8)$ & $6(31.6)$ & \\
\hline once fortnightly & $3(11.5)$ & - & $.001 \%$ \\
\hline once 3 weeks/month & $5(19.2)$ & - & \\
\hline Less & $8(30.8)$ & $3(15.8)$ & \\
\hline Intention-to-treat sample & $N=44$ & $N=42$ & \\
\hline $\begin{array}{l}\text { Treatment finished at } 4 \text { ye } \\
\text { (excluding drop-outs) }\end{array}$ & $12(27.3)$ & 5 (11.9) & .296 \\
\hline \multicolumn{4}{|c|}{ Using Psychotropic medication } \\
\hline Baseline & $34(77.3)$ & $30(71.4)$ & \\
\hline 3-years treatment & $23(52.3)$ & $23(54.8)$ & .507 \\
\hline Follow up & $22(50.0)$ & $24(57.1)$ & \\
\hline Treatment completers & $\mathbf{N}=32$ & $\mathbf{N}=\mathbf{2 1}$ & \\
\hline \multicolumn{4}{|l|}{ Completers' median number } \\
\hline sessions at 4 years & 222 & 292 & $<.001$ \\
\hline
\end{tabular}

$\dagger P$ Value based on Pearson Chi Square, except for $\mathcal{K}$, which was based on Mann whitney test.

\section{Treatment outcomes.}

The results of the primary and secondary outcome measures are shown in Table 2 and Figure 1 and 2. BPD-pathology was further reduced in both groups during the follow-up year, but this reduction was not significant compared to the 3-year assessment (SFT $P=.45$, TFP $P=.65$; but was significant compared to baseline: SFT MSTS $=-9.00, P<.001, d=2.72$; TFP MSTS $=-6.68, P<.001$, $d=2.06)$. Within each group, reductions on the BPDSI-IV subscale scores 
Table 2. Primary and Secondary Outcome Measures $(\mathrm{N}=86)$

\begin{tabular}{|c|c|c|c|}
\hline & $\begin{array}{c}\text { Schema Focused Group } \\
\text { No. (\%) }\end{array}$ & $\begin{array}{c}\text { Transference Focused Group } \\
\text { No. (\%) }\end{array}$ & $\begin{array}{c}P \\
\text { Valuet }\end{array}$ \\
\hline Recovery criterion 15 , yes & $23(52.3)$ & $12(28.6)$ & .035 \\
\hline \multirow[t]{2}{*}{ Reliable Change, yes } & $30(68.2)$ & $22(52.4)$ & .063 \\
\hline & Median (SE [95\%Cl]) & Median (SE [95\%CI]) & $\begin{array}{c}P \\
\text { Value } \ddagger\end{array}$ \\
\hline \multicolumn{4}{|l|}{$\begin{array}{l}\text { BPDSI-IV total score } \\
\text { (score range, } 0-90)^{*}\end{array}$} \\
\hline Baseline & $33.53(1.23[31.12-35.94])$ & $34.37(1.23[31.96-36.78])$ & \\
\hline 36-mo treatment & $16.24(1.51[13.28-19.19])$ & $21.87(1.71$ [17.95-25.79] ) & \\
\hline 1-year follow-up & $14.58(1.57[11.50-17.66])$ & $20.80(1.62[17.62-23.98])$ & $.004^{\mathrm{a}}$ \\
\hline \multicolumn{4}{|l|}{$\begin{array}{l}\text { EuroQol Thermometer } \\
\text { score (range } 0-100)^{* *}\end{array}$} \\
\hline Baseline & $50 \quad(3.29[43.55-56.45])$ & $55 \quad(2.72[49.67-60.33])$ & \\
\hline 36-mo treatment & $64.5(4.66$ [55.37-73.63]) & $67.5(2.91[61.80-73.20])$ & \\
\hline 1-year follow-up & $67.5(3.29[60.92-74.08])$ & $70 \quad(2.33[65.34-74.66])$ & $.648^{\mathrm{a}}$ \\
\hline \multicolumn{4}{|l|}{$\begin{array}{l}\text { WHOQOL total scores } \\
\text { (range } 4-20)^{* *}\end{array}$} \\
\hline Baseline & $10.33(0.19$ [ 9.96-10.70]) & $10.42(0.09$ [10.24-10.60]) & \\
\hline 36-mo treatment & $11.59(0.29$ [11.02-12.16]) & $11.09(0.19$ [10.72-11.46]) & \\
\hline 1-year follow-up & $11.88(0.44[11.00-12.76])$ & $11.41(0.26[10.89-11.93])$ & $.233^{\mathrm{a}}$ \\
\hline \multicolumn{4}{|l|}{$\begin{array}{l}\text { Psycho- \& Personality factor } \\
\text { score*** }\end{array}$} \\
\hline Baseline & $0.42(0.06[0.30-0.54])$ & $0.70(0.12[0.46-0.94])$ & \\
\hline 36-mo treatment & $-0.49(0.12[-0.73--0.25])$ & $0.19(0.18[-0.16-0.54])$ & \\
\hline 1-year follow-up & $-0.56(0.06[-0.68--0.44])$ & $0.13(0.19[-0.24-0.50])$ & $.001^{\mathrm{a}}$ \\
\hline $\begin{array}{l}\text { Abbreviation: SE, Standard } \\
\dagger P \text { Value based on binary } \\
\ddagger P \text { value based on Wilcox } \\
\text { a Endpoint Wilcox ANCOV } \\
* \text { Higher scores indicate more } \mathrm{s} \\
* * \text { Higher scores indicate } \mathrm{h} \\
* *{ }^{*} \text { Higher scores indicate } \\
\text { scores are slightly differen } \\
14 \text { instead of } 13 \text { assessme }\end{array}$ & $\begin{array}{l}\text {; Cl, Confidence interval. } \\
\text { ic regression. } \\
\text { OVA: robust ANCOVA }{ }^{41,42} \text { bas } \\
\text { medians. } \\
\text { BPD pathology. } \\
\text { levels of quality of life } \\
\text { psycho- and personality patho } \\
\text { the previous report }{ }^{23} \text { as thes }\end{array}$ & on Wilcox Rallfun package. & $\begin{array}{l}\text { 6-month } \\
\text { ted over }\end{array}$ \\
\hline
\end{tabular}

(corresponding to the 9 DSM-IV BPD-criteria) were maintained during the followup year $(P<.05$ on all BPDSI-IV criteria compared to baseline), further improvements on the subscales were not significant compared to the 3-year mark. Quality of life as assessed with the EuroQol thermometer, continued to increase in both groups during the follow-up year, but this increase was not significant compared to the 3-year assessment (SFT $P=.62$, TFP $P=.50$; compared to baseline: SFT MSTS $=3.43, P=<.001, d=1.03$; TFP MSTS $=4.19, P<.001$, $d=1.29$ ). WHOQOL-scores further improved during the follow-up year, (com- 
pared to baseline: SFT MSTS $=3.19, P=.001, d=0.96$; TFP MSTS=3.59, $P<.001, d=1.11$ ), but the improvement was not significant compared to the 3 year assessment (SFT $P=.59$; TFP $P=.32$ )
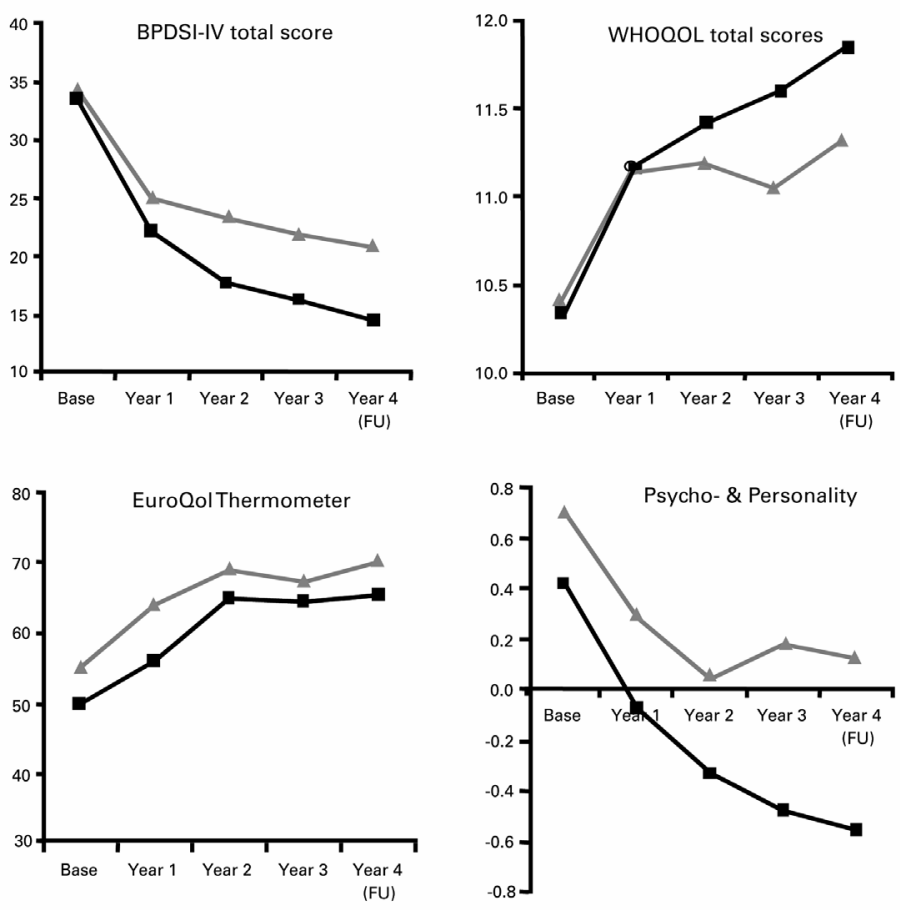

Figure 1. Graphs of medians of primary and secondary outcome measures $(N=86)$.

Note BPDSI-IV total scores, range 0-90; WHOQOL total scores, range 4-20; Euroqol thermometer scores, range 0-100; Composite score psycho- and personality factor scores. SFT (回) and TFP $(\Delta)$.

Logistic regression analysis on the BPDSI-IV recovery criterion at 1-year follow up with treatment group and baseline BPDSI-IV as predictors (covariates) showed a significant effect in favor of SFT (Wald $=4.45, P=.035$, OR 2.64 with CI[1.07-6.49]), baseline BPDSI-IV was N.S. $(P=.27)$. Without baseline BPDSI-IV the group effect was similar (Wald $=4.88, P=.027$, OR 2.74 with CI[1.12-6.69]). The SFT-effect became even stronger when psychotropic medication use at baseline and/or at 3-years, were additionally included as covariates (Wald $=5.34$, $P=.021$, OR 3.31 with $\mathrm{CI}[1.19-9.11])$, whereas psychotropic medication use at 3 -years is negatively associated with recovery (Wald $=5.43, P=.020$, OR 0.29 with CI[0.10-0.82]). BPDSI-IV baseline remained N.S. $(P=.17)$. All condition by 
covariate interactions failed to reach significance and were therefore not included in the final analyses.

Adding completion of the follow-up assessment (yes/no) as additional covariate to condition and psychotropic medication use at year 3 yielded the following results: SFT was still superior to TFP (Wald $=5.18, P=.023, \mathrm{OR}=3.08$ with $\mathrm{Cl}$ [1.17-8.13]), having completed follow-up assessments has no significant effect (Wald $=2.09, P=.15$, OR=2.04 with $\mathrm{Cl}[0.78-5.36]$ ), whilst medication use was still of negative influence (Wald $=6.69, P=.010, \mathrm{OR}=.28$ with $\mathrm{Cl}$ [0.11-0.73]). Thus having completed follow-up assessment did not influence SFT's superiority over TFP (OR in follow-up assessment completers $=3.10$; in non-completers $\mathrm{OR}=3.07$ ).
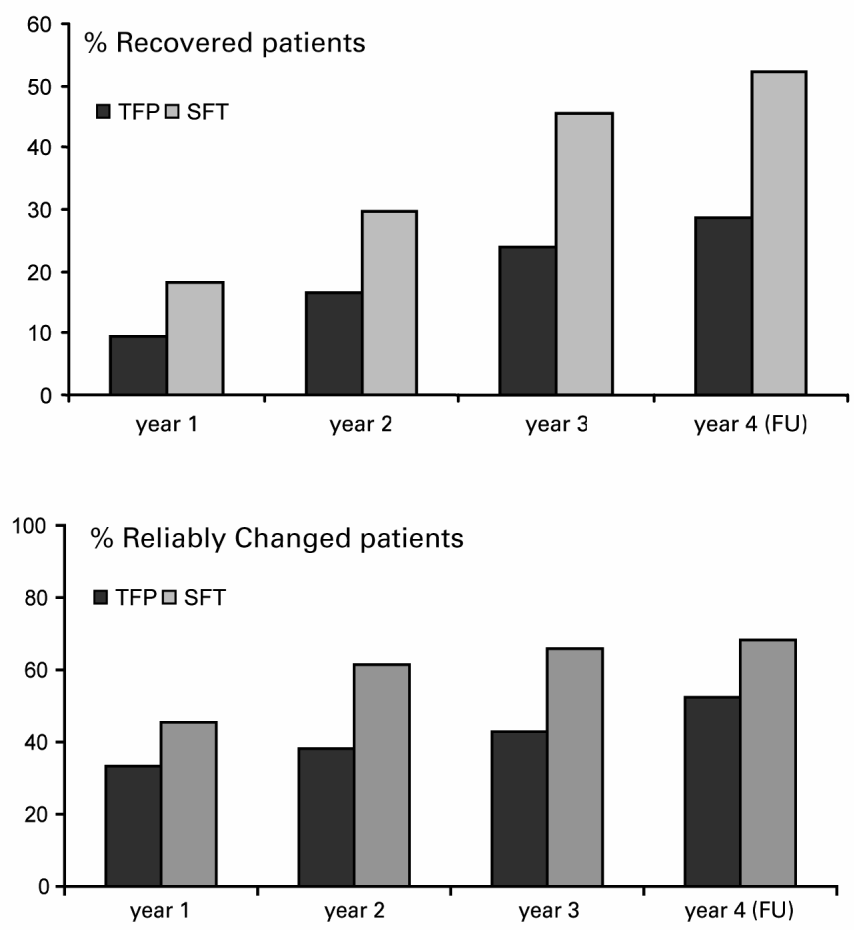

Figure 2. Percentages of recovered patients en reliably changed patients per year $(\mathrm{N}=86)$

When treatment drop-out (yes/no) was added as third covariate to condition and psychotropic medication use at year 3 , SFT just failed to reach significance compared to TFP (Wald=3.63, $P=.057, \mathrm{OR}=2.59$ with $\mathrm{Cl}$ [0.97-6.89]). Being a treatment drop-out was not of significant influence (Wald =1.91, $P=0.17, \mathrm{OR}=2.08$ with $\mathrm{Cl}[0.74-5.90])$, medication use remained of significant 
negative influence (Wald $=6.46, P=.011, \mathrm{OR}=.28$ with $\mathrm{Cl}[0.11-0.75]$ ). Thus taking into account treatment drop-out status somewhat reduced SFT's favorableness over TFP (OR in treatment completers $=2.78$; in non-treatment completers $O R=2.25$ ). Within the treatment completer group, the patients who terminated treatment before or at year 4 (follow-up) reached significantly more often the recovery criterion than the patients who were still in treatment (Wald $=6.18, P=.013, \mathrm{OR}=5.54$ with $\mathrm{Cl}[1.44-21.33]$ ). This result is in contradiction with the hypothesis that only patients who remain in treatment will achieve recovery.

Logistic regression analysis on reliable change status at 1-year follow-up revealed a trend for SFT (Wald $=3.45, P=.063, \mathrm{OR}=2.46$ with $\mathrm{CI}$.951-6.38]). As expected, baseline BPDSI-IV had a significant contribution to reliable change (Wald =8.46, $P=.004, \mathrm{OR}=1.11$ with $\mathrm{CI}[1.03-1.19]$ ). Higher baseline scores were associated with a larger chance to reach this criterion. When psychotropic medication use at baseline and/or 3-years were also included as predictors of reliable change at 1 -year follow-up, treatment group and baseline BPDSI-IV appeared as significant factors, not medication (SFT: Wald $=4.17, P=.041$, OR 2.74 with CI[1.04-7.20]; BPDSI-IV baseline: Wald=7.36, $P=.007$, OR 1.09 with CI[1.03-1.18]; baseline medication use $P=.71$; 3-years medication use $P=.29$ ). All condition by covariate interactions failed to reach significance and were therefore not included in the final analyses.

Adding having completed the follow-up assessment (yes/no) as additional predictor of reliable change yielded the following results; SFT's superiority over TFP just failed to reach significance (Wald $=3.63, P=.057, \mathrm{OR}=2.58$ with $\mathrm{Cl}$ [0.97-6.86]), having completed follow-up assessments was N.S. (Wald $=3.08$, $P=.08, \mathrm{OR}=2.37$ with $\mathrm{Cl}[0.90-6.19]$ ), whilst baseline BPDSI was of influence (Wald $=8.49, P=.004, \mathrm{OR}=1.12$ with $\mathrm{Cl}$ [1.04-1.19]). Thus, having completed follow-up assessments did only marginally influence SFT's superiority over TFP (OR in completers $=2.27$; in non-completers $O R=2.58$ ).

Results of the Wilcox ANCOVA on BPDSI-IV 1-year follow-up medians proved SFT to be more effective than TFP (MSTS $=3.55, P<.001, d=0.77$ ). When the patients who completed the follow-up assessment were analyzed separately, SFT again was superior to TFP (MSTS $=3.04, P=.002, d=0.93$ ). The median at follow-up reached by SFT-assessment completers was 11.79 , and 19.13 for TFP-assessment completers. SFT was also significantly more effective than TFP when the patients who dropped out of treatment were excluded (MSTS $=2.62, P=.008, d=0.72$; median of treatment completers at follow-up for SFT 12.49, for TFP 19.13). Within treatment completers we also analyzed condition effects in the two subsamples: patients still-in-treatment $(\mathrm{N}=31)$ and patients who finished therapy $(\mathrm{N}=22)$. Robust ANCOVA's on FU BPDSI-IV scores with BPDSI baseline as covariate using bootstrap technique revealed that 
in both samples SFT was superior to TFP, $P=.045$ (equivalent to $d=0.72$ ) and $P=.003$ (equivalent to $d=1.25$ ).

Wilcox robust ANCOVA tests at 1-year follow-up on all BPDSI-IV subscales medians demonstrated that SFT-patients had significantly larger improvements than TFP-patients with respect to abandonment, relationships, impulsivity, (para)suicidal behavior, emptiness, anger and dissociative \& paranoid ideation (all $P<.05)$. No significant group differences were found for identity disturbance and affective instability.

The Wilcox ANCOVA on both quality of life measures 1-year follow-up medians did not show a group effect (EuroQol thermometer MSTS $=0.46 P=.65$; WHOQOL MSTS $=1.21 P=0.23$ ).

Wilcox ANCOVA on medians of psycho- and personality pathology 1-year follow-up medians revealed a significantly larger effect for SFT than for TFP (MSTS $=3.21, P=.001, d=0.69$ ).

\section{Discussion}

After a 1-year naturalistic follow-up of the experimental 3-year treatment period, more BPD-patients treated with prolonged SFT and TFP sessions reached recovery or reliable change than at post test. The 1-year follow up also learned that improvements achieved with respect to BPD-pathology, experienced 'quality of life' and associated personality features were maintained. SFT remained favorable over TFP with respect to reduction in BPD-pathology, general psychopathology and change in SFT/TFP personality concepts. Differences previously found between treatments for quality of life-measures were no longer detected at follow-up. The significant difference in treatment intensity is of specific interest, demonstrating that SFT obtains better results than TFP with fewer sessions. In this respect it should be noted that the SFT protocols allows, and even indicates adjustments of treatment frequency such as gradual decrease of frequency and booster sessions. The TFP-protocol allows for less variation. Another noteworthy remark concerns the fact that all patients who continued treatment after 3 years remained with their therapists in the SFT- or TFP-model. Therefore, the naturalistic design of the 1-year follow-up study actually turned out to be a controlled design, making the SFT-TFP outcome comparison even more relevant.

Factors like treatment drop-out, FU assessment drop-out, and medication did not influence SFT's favorableness over TFP; Odds Ratios on the recovery criterion were in the 2.59-3.31 range (median 2.74). Analyses of the main outcome measure (BPDSI-IV) in subgroups revealed that SFT was superior to TFP in the intention-to-treat sample, in the FU assessment completers, in the treatment completers, in those that finished treatment regularly, and in those still in treat- 
ment, all with large effect sizes. Thus, the observed treatment effects seem not to depend of assessment or treatment status.

Efficacy of other treatments, like Dialectical Behavior Therapy (DBT) and Mentalization Based Treatment (MBT), is often assessed and manifested by good treatment retention, and reduced suicidal behavior, impulsivity and hospitalizations, not by covering the full range of BPD manifestations (Linehan, Armstrong, Suarez, 1991; Bateman \& Fonagy, 1999). Longer-term follow-up reports available for these treatments (Linehan, Heard, Armstrong, 1993; Bateman, Fonagy, 2001) read positively in that effects are maintained over time. The long-term perspective for BPD patients offered by more general, both retrospective and prospective, follow-up studies ranges from pessimistic to moderately optimistic (McGlashan, 1986; Paris, Brown, Nowlis, 1987; Sandell, Alfredsson, Berg et al, 1993; Stone, 1993; Links, Heslegrave, van Reekum, 1998; Paris, Zweig-Frank, 2001; Skodol, Gunderson, Shea et al, 2005; Zanarini, Frankenburg, Hennen et al, 2003). Comparing the present follow-up results to randomized studies on DBT and MBT is difficult, mostly due to differences in treatment aims (reduction of self-destructive psychopathology versus structural changes), settings (outpatient for DBT/SFT/TFP and partial hospitalization for MBT) and duration (1-year DBT, 1.5-year MBT, 3-year SFT/TFP). As important are differences in BPDpatient baseline characteristics with respect to self-destructive and parasuicidal behaviors. One-year follow-up of DBT-patients (Linehan, Heard, Armstrong, 1993) and 1.5-year follow-up of MBT-patients (Bateman, Fonagy, 2001) demonstrated maintenance of therapeutic effects, MBT-patients even continued to improve. DBT was primarily developed for treatment of severe (para)suicidal problems. MBT seems effective in severe partially hospitalized patients. SFT was found effective for both less and more severe patients, including self-injuring patients. Further research is needed to unravel which of the different treatments is most effective in subpopulations of BPD-patients. But note that the body of evidence for BPD-treatment effectiveness is still small. More well-designed BPDtreatment effectiveness studies, including other type of BPD-treatments and long-term follow-up are needed. An ongoing large randomized clinical trial comparing TFP, DBT and supportive psychotherapy forms a promising example (Clarkin, Levy, Lenzenweger et al, 2004). Uncontrolled studies may also be informative (e.g. Stevenson, Meares, D'Angelo, 2005; Nordahl \& Nysaeter, 2005).

A possible limitation of this study is the absence of a regular treatment-asusual (TAU) or no treatment condition. However, it is questionable whether such a condition as TAU for BPD-patients exists. The clinical impression is that the variety of treatment modalities and intensity of what supposedly is TAU, is very large and with little to no coherence. Comparisons of SFT, and also of TFP, to other manual-based treatments for BPD are more informative. 
Psychotropic medication use was not the focus of the present study but is of course associated and intertwined with mental health care for BPD-patients. The observation that patients can keep their psychotropic medication use reduced during follow-up is a positive one. One could hypothesize that this is also a result of patients' attained personality changes. The finding that psychotropic medication use was related to poorer outcome, but unrelated to BPD severity at baseline is noteworthy. The finding in a controlled study that adding a SSRI to DBT made DBT ineffective suggests that similar studies should be done with SFT and TFP (Simpson, Yen, et al., 2004). What factor is causing this relationship needs additional study.

Treatment termination was not linked to the recovery criterion but left to the therapist and patient. Most patients who achieved recovery status during their treatment continued therapy. Only after three years of treatment, feedback of individual assessment data was given to both patient and therapist, thereby examining treatments as close to clinical reality as possible without influencing therapist's and patient's motivation by the assessment results. It should be noted that the treatment intensity of many SFT-patients who recovered and reliably changed was already reduced as a result of the patient's experience of progress and/or the therapist's clinical evaluations. This may relate to the aim of both BPD-treatments to bring about significant fundamental personality change. Reaching 'symptomatic' recovery of BPD does not imply that all psychopathology and quality of life are at acceptable levels. Furthermore, actual changes in personality need time to consolidate. Other factors in the decision process of treatment termination are the patient's expectations, patient's dependence on 'being in treatment' and individual therapist's standards.

Although many patients still received treatment, it should be stressed that many of them received less intensive treatment, e.g. at year 4 more than $60 \%$ of the SFT-patients still receiving SFT had fewer sessions than once a week, about $50 \%$ once every 3 weeks or less (booster sessions). Even though this study supports the idea that long-term BPD-treatments are justified, it appears necessary to address and define guidelines for treatment termination that include different aspects (e.g. assessments of complaints, pathology, social functioning) and different perspectives (objective versus clinical, patient versus therapist). The availability of long-term treatments to BPD-patients is often negatively affected by governmental and health care providers' regulations. Demonstration of (cost-) effectiveness of such treatments may help to influence such regulation on a rational rather than a priori basis. Excluding all subjective aspects from clinical decisions is not realistic, but regulating treatment termination can potentially increase long-term treatment availability while remaining within reasonable limits of treatment duration. In this process, information on the cost-effectiveness of these treatments will be necessary, and is currently being assessed. 
The findings of this follow up study illustrate how difficult the decision can be to define the optimal treatment duration. Although originally the treatment was planned for 3 years, 27 out of the 44 SFT patients were still in treatment during year 4. If we limit ourselves to SFT and consider figure 1, different decisions would be reached depending upon the choice of criteria. As far as obtaining a BPDSI score below 15 is concerned, continuation into the fourth year seems defensible. This holds even more for the improvement of psychopathology, personality factors and quality of life, although the Euroqol seems to have reached it's optimum already after the second year. The present data do not support defining a 3 year period as the optimum treatment duration; rather they suggest that for a majority of the patients, treatment during a fourth year, albeit less frequently, may be indicated, if the aim is recovery and substantial improvement in psychopathology and personality pathology. A topic for future research would be which criterion, e.g. non-caseness or strong improvement of personality and psychopathology factors best predicts stability of the positive outcome.

Some recent large studies (Zanarini, Frankenburg, Hennen et al, 2003, 2005; Skodol, Gunderson, Shea et al, 2005) concluded that patients with BPD tend to remit in natural course, often, but not always, with intensive or sustained treatment. Future research should therefore explore whether lowerfrequent treatment can do as well as long-term treatment. Note also that in the present study a fine-graded dimensional interview was used to assess BPD manifestations. In our view, this gives a more valid indication of the degree of present BPD pathology than dichotomous criteria scores.

In conclusion, this study continues to contribute to a positive treatment perspective for BPD. As previously reported, future research recommendations focus on identifying active elements of SFT and TFP, and factors that facilitate treatment indication such as patient and therapist characteristics or their treatment expectations. A next step will be to examine whether the current findings can be generalized to regular non-academic and non-research mental health care settings. A first effort is made with an implementation study of SFT in general psychiatry (principal investigators: Marjon Nadort and Richard van Dyck). Another multicenter randomized trial testing SFT-effectiveness in patients with less impairing personality disorders will start in 2006 (principal investigator: Arnoud Arntz). 


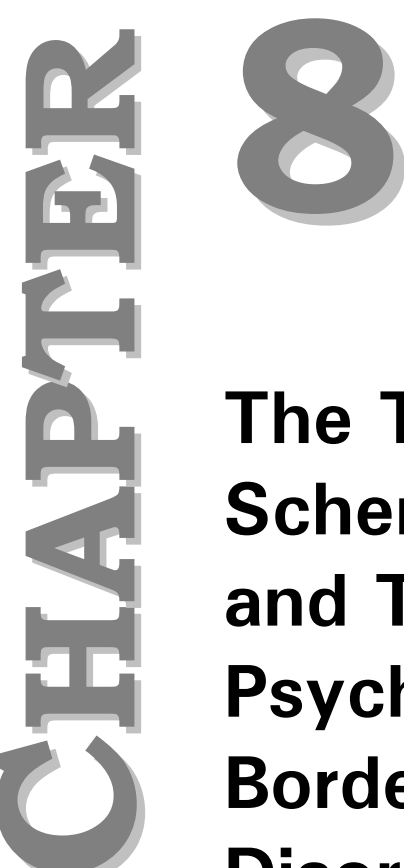

The Therapeutic Alliance in Schema Focused Therapy and Transference Focused Psychotherapy for Borderline Personality Disorder 


\section{Abstract}

This study investigated the quality and development of the therapeutic alliance as a mediator of change in Schema Focused Therapy (SFT) and Transference Focused Psychotherapy (TFP) for borderline personality disorder. 78 Patients were randomly allocated to 3-years SFT or TFP with sessions twice a week. Scores for the therapeutic alliance were higher in SFT than in TFP. Negative ratings of therapists and patients at early treatment were predictive of dropout, while increasingly positive ratings of patients in the first half of treatment predicted subsequent clinical improvement. Dissimilarity between therapist and patients in pathological personality characteristics had a direct effect on growth of the therapeutic alliance. It is concluded that the therapeutic alliance is an important common therapy factor critically affected by type of treatment. 


\section{Introduction}

The therapeutic alliance can be defined as the quality of involvement between therapist and patient, as reflected in their task teamwork and personal rapport (Orlinsky, Ronnestad, \& Willutzki, 2004). The quality of the alliance proves to be consistently associated with a positive outcome across different forms of psychotherapy. Especially the therapist's contribution to the alliance as rated by patients shows a consistent although modest relationship with outcome (Martin, Garske, et al., 2000; Orlinsky, Ronnestad, et al., 2004). Although, the therapeutic alliance seems to be a common therapy factor crucial to the change process across different therapeutic orientations, comparative research of the quality of the therapeutic alliance is necessary to detect possible similarities and differences across orientations.

The results of studies investigating the therapeutic alliance across different treatment modalities in mostly heterogeneous groups of patients with an Axis I disorder are equivocal (e.g. Brunink \& Schroeder, 1979; Marmar, Gaston, Gallagher, et al., 1989; Raue, Goldfried, \& Barkham, 1997; Sloane, Staples, Cristol, et al., 1975). Nevertheless, the available evidence suggests that compared to psychodynamic psychotherapy the alliance in cognitive-behavior therapy is more characterized by supportive communication, expressed sympathy and interpersonal contact. It is conceivable that there are more pronounced differences in the therapeutic alliance in the treatment of personality disorders instead of Axis I disorders. From a cognitive-behavioral perspective on the treatment of personality disorders a closer, warmer therapeutic alliance is necessary than in the treatment of an acute Axis I disorder such as anxiety or depression (Beck, Freeman, et al., 1990; Beck, Freeman, et al., 2004). Much of the therapist's role consists of a process of re-education and in the course of time the therapist even becomes a role model for the patient. In schema-focused therapy, therapists even try to provide 'limited reparenting' in order to meet partially the unmet emotional needs in order to develop more healthy schemas (Young, Klosko, et al., 2003). This is in contradiction to some psychodynamic approaches in which the 'real relationship' is considered to be on a path to boundary violation, anti-therapeutic, and a probable manifestation of countertransference enactment (Bateman \& Fonagy, 2004). Especially in transference focused psychotherapy the potential of intense negative transference reactions to disturb the therapeutic alliance is emphasized and accordingly the need to interpret these negative reactions early in treatment (Clarkin, Yeomans, et al., 1999). Only in a more advanced stage of treatment the patient is expected to have gained a more empathic, realistic impression of the therapist as a person.

Up till now, no longitudinal research is available into different alliance qualities between psychodynamic versus cognitive-behavioral therapy in the treat- 
ment of personality-disordered individuals. This study tries to advance earlier comparative research of the therapeutic alliance (a) by investigating a homogeneous group of patients with a borderline personality disorder (BPD), (b) by using two well-defined forms of cognitive-behavioral and psychodynamic therapy with dissimilar therapeutic alliance qualities, and (c) by studying the development of the therapeutic alliance during treatment.

The consistent relationship of the quality of the therapeutic alliance with outcome can be interpreted in different ways. Outcome and alliance measures may be confounded and the quality of the alliance may even be a mere epiphenomenon of positive treatment change. However, in many studies, evidence for the therapeutic alliance as a mediator of change has been collected showing that outcome can be predicted from early alliance ratings (e.g. Gaston, Marmar, Gallagher, et al., 1991; Salvio, Beutler, Wood, et al., 1992). Although it may be expected that in the treatment of personality disordered individuals early rating of the therapeutic alliance will predict premature termination and outcome, it is conceivable that in addition growth of the therapeutic alliance during the first phase of treatment will facilitate later outcome. Especially in the treatment of BPD patients the establishment of a therapeutic relationship is not readily accomplished since most of these patients' problems are generally manifested in the interpersonal realms. So the development and maintenance of a collaborative therapeutic alliance during the first year of treatment as a prerequisite for further treatment is seen as one of the central issues of intensive long term treatment across different psychotherapeutic orientations (Beck, Freeman, et al., 1990; Clarkin, Yeomans, et al., 1999).

The investigation of the intertwined and sequential relationship between alliance and client's improvement during treatment is seen as an advancement compared to the research into early alliance scores as predictors of later outcome (Barber, Connolly, Crits-Christoph, et al., 2000). Up till now no systematic research on the predictive relationships among changes in the therapeutic alliance and outcome in different phases of long term treatment of personality disorder has been reported. Consequently, the purposes of the present study is not only to analyze whether (a) the quality of the therapeutic alliance at early treatment predicts dropout and outcome, but also (b) whether there is any support of a causal role of growth of the therapeutic alliance during the first phase of treatment in facilitating later outcomes.

In psychotherapy outcome research, the major focus is on comparing the effectiveness of various theoretical and technical approaches, while trying to control, reduce or eliminate the influence of therapist factors by constructing treatment manuals that ideally can be applied in an identical manner to all patients within a particular diagnostic category by any therapist (Lambert, 1989). As a result, relatively little attention has been given to the therapeutic impact of 
discrete therapist variables such as age, experience and training, ethnicity, and gender, and only a few studies have examined therapist variables in a relational or interpersonal context (Beutler, Malik, Alimohamed, et al., 2004).

Particularly with respect to personality traits, research has tried to define ways in which therapist and patient qualities mutually interact, resulting in patterns of match and mismatch (Beutler, Malik, et al., 2004). Two opposing viewpoints have been developed. The conventional presupposition that similarity in personality traits increases the attachment and participation in treatment and consequently promotes outcome has been confirmed in some studies (e.g. Herman, 1998), but support for the opposite presupposition has also been found (e.g. Berry \& Sipps, 1991). In this view it is argued that if therapist's and patient's dysfunctional personality characteristics are harmoniously blended, this will result in a therapist's blind spot severely hampering an adequate understanding of his or her emotional reactions to the patient and controlling these in the therapeutic relationship.

It is well known that the therapeutic alliance can be extremely complicated in the treatment of BPD (Beck, Freeman, et al., 1990; Yeomans, Clarkin, et al., 2002; Young \& Klosko, 1994). Both within a cognitive and psychodynamic perspective, personal qualities of the therapists in their reciprocal relationship with the personal qualities of the patient are assumed to be responsible for the quality and development of the therapeutic alliance. Activation of therapists' cognitive schema's by the expression of similar early maladaptive schema's in patients (Beck, Freeman, et al., 1990; Young, Klosko, et al., 2003) or unconscious countertransference reactions elicited by the patient's primitive projective mechanisms (Clarkin, Yeomans, et al., 1999) may preclude the functional processing of "transference" reactions in the therapeutic relationship. Therefore, it is to be expected that the match of pathological personality characteristics of therapists and patients will impede the development of the therapeutic alliance

By examining dissimilarity in pathological personality characteristics between therapists and patients, the development of the therapeutic alliance and clinical outcome within one comprehensive analysis, the present study tries to improve on earlier research on the impact of therapists' personality traits on outcome (a) by assessing pathological personality traits of relevance for borderline personality disorder; (b) by studying these traits in an interpersonal context; and (c) by investigating whether therapist-patient similarity in pathological personality characteristics indirectly impacts outcome by its direct effect on the therapeutic alliance.

In sum, the purpose of the present study was to investigate the following predictions: (a) the quality of the therapeutic alliance is rated higher in SFT than in TFP; (b) a lower quality of the therapeutic alliance at early treatment predicts premature treatment termination and clinical outcome; (c) growth of the thera- 
peutic alliance during the first year of therapy facilitates later clinical improvement; (d) dissimilarity in pathological personality characteristics between therapists and patients facilitates the development of the therapeutic alliance and indirectly affects therapy outcome.

\section{Methods}

The present study was conducted as part of a multicenter randomized trial, two groups design. For a fuller description of the methods and results of this trial the reader is referred to Giesen-Bloo, van Dyck, et al. (2006).

\section{Design}

Patients' first assessment was made after inclusion and before random allocation to treatment conditions. Then assessments were made every three months for three years. Primary outcome measures were administered at each assessment. The measurements for the quality of the therapeutic alliance were only collected after three months (i.e. early treatment), after 15 months (i.e. midtreatment) and after 33 months (i.e. late treatment). Personality assessments of patients took place at baseline, while therapists answered the personality questionnaires three months after the start of therapy.

Independent trained research assistants assessed patients. Patients diagnoses were assessed with the SCIDs, semi-structured clinical interviews for the Diagnostic and Statistical Manual of Mental Disorders, Fourth Edition (DSM-IV; First, Gibbon, et al., 1997; First, Spitzer, et al., 1996). Patients were also screened with a semi-structured clinical interview, the Borderline Personality Disorder Severity Index, fourth version (BPDSI-IV; Arntz, van den Hoorn, et al., 2003; Giesen-Bloo, Wachters, et al., 2006). A BPDSI-IV cutoff score of 20 (range 0-90) discriminates BPD patients from other personality pathology patients. Signed informed consent was obtained after full explanation of procedures and of both therapies, before the first assessment and randomization. Study researchers, therapists and research assistants had no foreknowledge of treatment allocation. The study protocol was approved by the medical ethical committees of the four participating centers.

\section{Patients and Settings}

Patients were referred by mental health institutes. Inclusion criteria were a main diagnosis of BPD, age between 18 and 60 years, a BPDSI-IV score above 20 and Dutch literacy. General exclusion criteria were psychotic disorders (except short, reactive psychotic episodes), bipolar disorder, dissociative identity disorder, antisocial personality disorder, attention deficit hyperactivity disorder, 
addiction of such severity that clinical detoxification was indicated (after which entering treatment was possible), psychiatric disorders secondary to medical conditions and mental retardation.

\section{Treatment Conditions and Therapists}

Both treatments were administered in biweekly 50 minutes sessions. Treatment protocols addressed the theoretical model, treatment frame, different phases and the use of strategies and techniques of SFT (Young \& Klosko, 1994; Young, Klosko, et al., 2003) and TFP (Clarkin, Yeomans, et al., 1999; Yeomans, Clarkin, et al., 2002). Jeffrey Young PhD (SFT) and Frank Yeomans MD PhD (TFP) initially trained the participating therapists. Weekly local intervision with 45 SFT or TFP therapists, 4-monthly 1-day central supervision and 9-monthly 2day central supervision by Jeffrey Young (SFT) or Frank Yeomans (TFP) was provided in the study. Treatment integrity was assessed by independent rating of audiotapes of randomly selected sessions of each trimester for each patient. Adherence and quality to the SFT and TFP manuals was good.

\section{Assessment}

Borderline Personality Disorder Severity Index (BPDSI-IV). The primary outcome measure, the BPDSI-IV, is a DMS-IV BPD criteria based semi-structured interview and forms a quantitative index of the current severity and frequency of specific BPD symptoms (Arntz, van den Hoorn, et al., 2003). The interview covers a period of three months, is suitable for use as a treatment outcome measure and showed excellent (interrater) reliability, validity and sensitivity to change. The internal consistency of the BPDSI-IV total score was 0.83 .

Working Alliance Inventory (WAI). The WAI (Horvath \& Greenberg, 1989) consists of three subscales of 12 items each, rated on a 5-point Likert-type scale ranging from 1 ("never") to 5 ("always"). The subscales address agreement about the goals of therapy, agreement about the tasks of therapy, and the bond between the client and therapist. Patients completed the patient form (WAI-P) measuring the contribution of the therapist to the alliance as perceived by the patient and therapists completed the therapist form (WAI-T) in which they rated the contribution of the patient to the alliance. Because of the high intercorrelations among subscales (WAI-P range: 0.69-0.88; WAI-T range: 0.67-0.89) subscale mean scores were added together to derive a global score. A higher score on the WAI indicates a higher quality of the working alliance. The internal consistency of the WAI-P was 0.94 and of the WAI-T 0.95.

Difficult Doctor-Patient Relationship Questionnaire - Ten Item Version (DDPRQ-10). The DDPRO is a 10-item self-report questionnaire, which aims to measure the extent to which patients are experienced as frustrating or difficult in the therapeutic relationship by their doctor or therapist and provoke levels of dis- 
tress that transcend the expected and accepted level of difficulty (Hahn, Thompson, Stern, et al., 1990). The items are answered on a 6-point Likert-type scale ranging from 1 ("not at all") to 6 ("a great deal"). The total score equals the mean of the 10 items. A higher score indicates a higher level of therapist frustration. The internal consistency of the DDPRO was 0.79 .

Young Schema Questionnaire (YSQ). The YSO is a 205-item self-report questionnaire developed to measure 16 core beliefs or early maladaptive schemas (Schmidt, Joiner, et al., 1995). The items are answered on a 6-point Likerttype scale ranging from 1 ("totally inapplicable to me") to 6 ("describes me perfectly"). The sixteen core beliefs are: (1) Abandonment/instability; (2) Defectiveness/shame; (3) Emotional deprivation; (4) Mistrust/abuse; (5) Social isolation; (6) Dependence/ incompetence; (7) Vulnerability to harm and illness; (8) Enmeshment; (9) Failure to achieve; (10) Social undesirability; (11) Entitlement/grandiosity; (12) Insufficient self-control/self-discipline; (13) Self-sacrifice; (14) Subjugation; (15) Emotional inhibition; and (16) Unrelenting standards. Item mean scores were calculated for each scale. A higher score on a scale indicates a higher endorsement of dysfunctional core beliefs. Subscale mean scores were summed up to derive a global score for dysfunctional core beliefs. The YSO was found to be valid and reliable in a study by Rijkeboer, van den Bergh, et al. (2005). Internal consistencies of the YSO subscales in the present study varied from 0.77 to 0.94 in the therapist sample ( 0.99 for the total scale) and from 0.82 to 0.93 in the patient sample (0.98 for the total scale).

Inventory of Personality Organization (IPO). A shortened 90-item IPO was derived from the 155-item IPO (Lenzenweger, Clarkin, et al., 2001). The 90-item IPO consists of three primary clinical and two secondary interpersonal relations scales. The IPO items have a 5-point Likert-type scale ranging from 1 ("never true") to 5 ("always true"). The three primary clinical scales relevant to the central dimensions of Kernberg's personality organization model (i.e., reality testing (13 items), identity diffusion (17 items), and primitive psychological defenses (14 items)) have been psychometrically investigated and display adequate internal consistency and good test-retest reliability (Lenzenweger, Clarkin, et al., 2001). Item mean scores for each of these subscales were calculated. A higher score on a scale indicates a higher level of this pathological personality characteristic. Subscale mean scores were added together to derive a global score for impairments in personality organization. Internal consistencies of these three IPO subscales in the present study varied from 0.84 to 0.88 in the therapist sample ( 0.95 for the total scale) and from 0.82 to 0.86 in the patient sample $(0.93$ for the total scale). 


\section{Statistical analyses}

Differences in the quality and development of the therapeutic alliance between treatment conditions were analysed with a 2 (group) $\times 3$ (time) mixed factorial design with repeated measures on the second factor.

Because in previous research a clinical cutoff score of 15 discriminated between BPD patients and non-patient controls (Arntz, van den Hoorn, et al., 2003), with a sensitivity of 1 and specificity of 0.97 , the recovery criterion was therefore defined as a BPDSI-IV score of less than 15, and maintaining this score till the last assessment. A second success criterion used, was the Jacobson and Truax reliable change index (Jacobson \& Truax, 1991). For the BPDSI-IV, reliable change was achieved when a reduction of at least 11.70 was achieved. Using logistic regression analyses it was investigated to what extent early treatment WAI-P, WAI-T and DDPRO scores predicted these two outcome criteria above and independent of pre-treatment BPDSI scores. To detect time to dropout, survival analyses were conducted using a proportional hazard approach to survival analysis (Cox regression) with dropout as the dependent variable and pre-treatment BPDSI and early treatment process variables as independent variables.

To determine whether early- to midtreatment changes in process variables predicted mid- to late treatment changes in outcome, cross-lagged correlations among residualized change scores were calculated (Finkel, 1995). When a correlation between early process changes and later outcome changes was statistically significant, hierarchical regression analyses were performed to test whether early process changes still predicted later outcome changes after controlling for autocorrelations (i.e. the correlations between early and late process changes) and synchronous correlations (i.e. the correlations between early process and early outcome changes) (cp. Burns, Kubilus, Bruehl, et al., 2003; Evon \& Burns, 2004). Regressions were also used to determine the inverse association.

Differences between therapists and patients in the profile of cognitive schema's or personality organisation were analysed according to Cronbach and Glesser (Cronbach \& Gleser, 1953). Therapist-patient difference values were computed by using YSO or IPO subscale scores corrected for elevation and scatter. The $D \ni^{2}$ statistic represents the sum of the squared differences on the subscales of the YSO or IPO. The larger the obtained value, the greater the degree of dissimilarity between therapist and patient. In order to test the causal model linking dissimilarity in pathological personality characteristics, development of the therapeutic alliance, and outcome, the analytic strategy as recommended by Baron \& Kenny (1986) was used.

Statistical analyses were performed in the intention-to-treat and the completers sample. An intention-to-treat approach was applied, with either using last clinical endpoint during the 3-year treatment period or using the last clinical endpoint carried forward for trend analyses. Missing data were substituted using a 
last-observation-carried-forward method. In addition, a completers analysis was performed. All tests were interpreted with a significance level of $5 \%$. The Statistical Package for Social Sciences, version 11.0 for Windows, was used for all analyses.

\section{Results}

\section{Participants}

From the 86 patients included in the randomized controlled trial comparing SFT and TFP (Giesen-Bloo, van Dyck, et al., 2006) eight patients in TFP compared to none in SFT terminated treatment prematurely between pre-treatment and the first repeated assessment after three months (Fisher's exact test, $p<.01$ ). In two patients it did not prove to be possible to agree upon a treatment contract, which in TFP is considered to be a prerequisite for starting treatment, and in one patient TFP was contra-indicated according to the subjective evaluation of the therapist. Five other patients dropped out in the first three months of treatment after completing treatment contracting. Although no patients' WAI-P scores of the five dropouts from TFP in this period are available, therapists' WAI-T and DDRPO have been collected. Compared to patients still in treatment at early treatment $(n=78)$ (see Table $2)$, the quality of the therapeutic alliance on the WAI-T was rated as significantly lower $(\mathrm{M}=8.4 ; \mathrm{Sd}=1.1 ; \mathrm{t}(60)=3.67, p<.01)$, and these patients were also rated as significantly more irritating and frustrating in the therapeutic relationship on the DDPRO ( $\mathrm{M}=3.5 ; \mathrm{Sd}=.9 ; \mathrm{t}(60)=2.14, p<.05)$.

Table 1 gives an overview of the biographical and clinical characteristics of the 78 patients in treatment at early treatment, which constitute the intentionto-treat sample of the present study. Chi-square analyses and t-tests for independent samples revealed no significant differences with respect to biographical and clinical characteristics between the 34 patients in the TFP and 44 in SFT condition (all $p>.1$ ).

Forty-four therapists (21 in TFP and 23 in SFT) participated in the randomized controlled trial of TFP compared to SFT. Three therapists held Doctoral degrees, 37 therapists held Masters degrees, and four therapists held Bachelor degrees with postgraduate training. All therapists had prior therapy experience in the associated therapeutic orientation and clinical experience in treating patients with a borderline personality disorder. The ratio of male to female therapist was 1:1. Thirty-two therapists were clinical psychologist, 7 were psychiatrist and five were social workers or psychiatric nurses with an advanced psychotherapeutic training. Thirteen therapists treated one patient, 28 therapists two patients and 3 therapists three patients of the present intention-to-treat sample $(n=78)$. Of the 44 therapists participating in the multicenter randomized trial, 30 (68.2\%) 
Table 1. Biographical and Clinical Characteristics (theoretical ranges between parentheses)

\begin{tabular}{lrrrr}
\hline Variable & \multicolumn{2}{c}{$\begin{array}{c}\text { Schema Focused Therapy } \\
(\mathrm{n}=44)\end{array}$} & $\begin{array}{c}\text { Transference Focused Psycho- } \\
\text { therapy }(\mathrm{n}=34)\end{array}$ \\
\hline & $\mathrm{M}$ & $\mathrm{SD}$ & $\mathrm{M}$ & $\mathrm{SD}$ \\
\cline { 2 - 5 } & & & & \\
Age & 31.7 & 8.9 & 29.4 & 6.5 \\
BPDSI (0-90) & 33.1 & 7.1 & 35.4 & 9.1 \\
N Axis I diagnoses & 2.9 & 1.5 & 2.4 & 1.7 \\
N Axis II diagnoses incl. BPD & 2.1 & 1.2 & 2.1 & 1.3 \\
YSO global score (16-96) & 53.5 & 11.9 & 58.8 & 12.2 \\
IPO global score (3-15) & 8.8 & 1.9 & 8.8 & 1.8 \\
& $\mathrm{No}$ & $\%$ & No & $\%$ \\
Gender (women) & 40 & 90.9 & 32 & 94.1 \\
Education & & & & \\
$\quad$ Graduate/professional & 6 & 13.6 & 2 & 9.5 \\
$\quad$ College graduate & 3 & 6.8 & 6 & 16.7 \\
$\quad$ Some college & 17 & 38.6 & 14 & 33.4 \\
$\quad$ High school graduate & 5 & 11.4 & 6 & 23.8 \\
$\quad$ Grades 7-11 & 13 & 29.6 & 6 & 16.7 \\
\hline
\end{tabular}

Note. $\mathrm{BPDSI}=$ Borderline Personality Disorder Severity Index; YSQ=Young Schema Questionnaire; IPO = Inventory of Personality.

(13 in TFP and 17 in SFT) also gave their informed consent to participate in the present subsidiary study on the therapeutic alliance in the treatment of borderline personality disorder and were willing to answer both the YSO and IPO concerning their own personality characteristics and the WAI-T and DDPRO with respect to the patients which they were treating. Chi-square analyses revealed no significant differences in gender, professional background, therapy condition or number of patients in treatment (all $p>.1$ ) between these therapists and the remaining 14 therapists.

These 30 therapists rated the therapeutic alliance of the 57 of the 78 patients which they treated with SFT or TFP $(73.1 \%$ of the intention-to-treat sample of 78 patients). Chi-square analyses and t-tests for independent samples revealed no significant differences with respect to biographical and clinical characteristics, WAI-P and BPDSI scores between these 57 patients versus the 29 patients which were treated by the other 14 therapists and for which no therapist ratings are available (all $p>.1$ ).

Of the 78 patients with early WAI-P scores and still in treatment at mid and late treatment, data on the middle and late alliance were available for $66(100 \%)$ and $52(98.1 \%)$ patients, respectively. Of the 57 patients with early alliance ratings by the therapist and still in treatment at mid and late treatment, WAI-T and 
DDPRO scores of the middle and late alliance were available for $42(85.7 \%)$ and $33(86.8 \%)$ of the patients at mid and late treatment.

\section{Early- to Mid- to Late Treatment Changes}

Table 2 summarizes early-, mid- and late treatment values for the process and outcome variables. In analysing BPDSI scores $(n=78)$ a significant effect for time, $(F(1,76)=41.54, p<.001)$ was found. Planned comparisons revealed that BPDSI scores changed significantly form early- to midtreatment and also from mid- to late treatment. No significant effect for Group or a significant Group $X$ Time interaction was observed.

In analysing WAI-P scores $(n=78)$ a significant effect for group, $(F(1,76)=6.00, p<.05)$, and time, $(F(1,76)=7.15, p<.01)$ was found. Planned comparisons showed that WAI-P scores changed significantly from early to late treatment. No significant effect for the Group $X$ Time interaction was observed. In analysing WAI-T scores $(n=57)$ only a significant effect for group $(F(1,55)=9.23, p<.01)$ was observed. No significant main effect for time or Group $X$ Time interaction was observed. In analysing DDPQR scores $(n=57)$ a significant effect for group $(F(1,55)=15.25, p<.001)$ and significant interaction of Group X Time $(F(1,55)=6.32, p<.05)$ was observed. No significant main effect for Time was observed.

Table 2. Early-, Mid-, and Late Treatment Values for Process and Outcome Variables (theoretical ranges between parentheses)

\begin{tabular}{|c|c|c|c|c|c|c|c|c|c|c|c|c|}
\hline \multirow{3}{*}{ Variable } & \multicolumn{6}{|c|}{ Schema Focused Therapy } & \multicolumn{6}{|c|}{ Transference Focused Psychotherapy } \\
\hline & \multicolumn{2}{|c|}{ Early } & \multicolumn{2}{|c|}{ Mid } & \multicolumn{2}{|c|}{ Late } & \multicolumn{2}{|c|}{ Early } & \multicolumn{2}{|c|}{ Mid } & \multicolumn{2}{|c|}{ Late } \\
\hline & M & SD & M & $\mathrm{SD}$ & $\mathrm{M}$ & SD & M & SD & $\mathrm{M}$ & SD & M & SD \\
\hline $\begin{array}{l}\text { BPDSI }{ }^{a} \\
(0-90)\end{array}$ & 27.4 & 9.5 & 21.3 & 11.1 & 18.5 & 11.6 & 29.2 & 7.9 & 24.5 & 9.7 & 22.7 & 12.1 \\
\hline $\begin{array}{l}\text { WAI-Pa } \\
(3-15)\end{array}$ & 11.0 & 1.5 & 11.5 & 1.6 & 11.5 & 1.7 & 10.2 & 1.9 & 10.4 & 1.8 & 10.7 & 2.0 \\
\hline $\begin{array}{l}\text { WAI-T }^{b} \\
(3-15)\end{array}$ & 11.1 & 1.2 & 11.0 & 1.2 & 11.2 & 1.4 & 10.1 & 1.4 & 10.1 & 1.5 & 10.0 & 1.5 \\
\hline $\begin{array}{l}\operatorname{DDPRQ}^{\mathrm{b}} \\
(1-6)\end{array}$ & 2.6 & 0.6 & 2.6 & 0.7 & 2.4 & 0.8 & 3.0 & 0.7 & 3.3 & 0.7 & 3.3 & 0.8 \\
\hline
\end{tabular}

Note. Early=early-treatment; Mid=midtreatment; Late=late treatment; BPDSI=Borderline Personality Disorder Severity Index; WAI-P =Working Alliance Inventory- Patient Version; WAI$\mathrm{T}=$ Working Alliance Inventory- Therapist Version; DDPRQ = Difficult Doctor-Patient Relationship Questionnaire; ${ }^{a} \mathrm{n}=78$ (44 in SFT and 34 in TFP); ${ }^{\mathrm{b}} \mathrm{n}=57$ (35 in SFT and 22 in TFP)

Overall, these results suggest that quality of the therapeutic alliance is rated higher in SFT than in TFP by therapists as well as patients. Moreover, the quality of the therapeutic alliance as rated by the patient increases in the course of 
treatment irrespective of treatment condition, while therapist frustration decreased in SFT but increased in TFP.

In order to determine whether the differences in WAI-P, WAI-T and DDPOR scores between treatment conditions may be due to clinical improvement as achieved in the first three months of treatment, first baseline BPDSI scores at the start of treatment were regressed to 3-months early-treatment scores to form residualized change scores. Next, early-treatment WAI and DDPRO scores were analysed with one-way ANOVA's with residualized BPDSI change scores as a covariate. Also after statistically controlling for any changes in BPDSI scores between baseline and early treatment, the differences between conditions in WAI-P scores $(F(1,75)=3.94, p<.05)$, WAI-T scores $(F(1,54)=8.54, p<.01)$, and DDPRO scores $(F(1,54)=5.57, p<.05)$ between SFT and TFP remained statistically significant.

\section{Prediction of Three-Years Outcome by Early Treatment Process Variables}

At the $13^{\text {th }}$ assessment three years after the start of treatment, 20 of the 44 patients in SFT $(45.5 \%)$ and 10 of the 34 patients in TFP $(29.4 \%)$ reached the BPDSI recovery criterion (a score on the BPDSI of less than 15). With respect to the reliable change index (a change of at least 11.70 on the BPDSI), 29 of the 44 patients in SFT $(65.9 \%)$ and 17 of the 34 patients in TFP (50.0\%) reached this criterion after 3 years of treatment. Chi-square analyses revealed no significant association of treatment condition with either the BPDSI recovery or reliable change criterion (all p-values $>.10$ ). Table 3 gives an overview of the WAI-P, WAI-T and DDPRO scores at early treatment of patients divided on the basis of reaching the BPDSI recovery or reliable change criterion.

Using separate logistic regression analyses with the BPDSI recovery or reliable change criterion as dependent variables and BPDSI pretreatment scores, treatment condition and early treatment process scores as independent variables, neither early treatment patients' ratings (WAI-P scores) nor early treatment therapists' ratings of the therapeutic alliance (WAI-T and DDPRQ) were predictive of clinical improvement after three years of treatment.

Furthermore, it was investigated whether premature treatment termination was related to the quality of the therapeutic alliance at early treatment. From early treatment (after three months) to midtreatment (after 15 months) 12 patients dropped out of treatment ( 7 in TFP and 5 in SFT). In the period between midtreatment and late treatment (after 33 months) a further 13 patients dropped out (6 in TFP and 7 in SFT). There was no significant association of treatment condition with dropout rate in this particular treatment period. Separate Cox regression analyses with BPDSI pretreatment scores, treatment condition and early treatment process scores as independent variables revealed a significant effect for 
Table 3. Ratings of the Therapeutic Alliance at Early Treatment of Recovered versus Not-Recovered and Reliably Changed versus Not-Reliably Changed Patients (theoretical ranges between parentheses)

\begin{tabular}{|c|c|c|c|c|}
\hline \multirow[t]{2}{*}{ Variable } & $\mathrm{M}$ & SD & $\mathrm{M}$ & SD \\
\hline & Not-Recovered & \multicolumn{3}{|c|}{ Recovered } \\
\hline WAI-P a & 10.4 & 1.8 & 11.2 & 1.4 \\
\hline WAI-T ${ }^{b} \quad(3-15)$ & 10.7 & 1.4 & 10.7 & 1.3 \\
\hline \multirow[t]{2}{*}{ DDPRO $^{\mathrm{b}} \quad(1-6)$} & 2.8 & 1.7 & 2.7 & 0.6 \\
\hline & \multicolumn{2}{|c|}{ Non-Reliably Changed } & \multicolumn{2}{|c|}{ Reliably Changed } \\
\hline WAI-P ${ }^{c} \quad(3-15)$ & 10.2 & 1.8 & 11.0 & 1.6 \\
\hline WAI-T d (3-15) & 10.5 & 1.3 & 10.9 & 1.4 \\
\hline DDPRO $^{d} \quad(1-6)$ & 2.8 & 0.7 & 2.7 & 0.6 \\
\hline
\end{tabular}

Note.WAI-P $=$ Working Alliance Inventory- Patient Version; WAI-T = Working Alliance InventoryTherapist Version; DDPRQ = Difficult Doctor-Patient Relationship Questionnaire; ${ }^{a} \mathrm{n}=78$ (48 notrecovered vs 30 recovered); ${ }^{b} \mathrm{n}=57$ (35 not-recovered vs 22 recovered); ${ }^{c} \mathrm{n}=78$ (32 notreliably changed vs 46 reliably changed) ; ${ }^{d} n=57$ (26 not-reliably changed vs 31 reliably changed)

early treatment WAI-P scores $(n=78)$, (Wald $(1)=4.379, p<.05$, Hazard ratio $=.775,95 \% \mathrm{Cl}=.610-.984)$. Moreover, especially therapists' early treatment WAI-T and DDPRO scores $(n=57)$ proved to be predictive for time to dropout (Wald $(1)=8.171, p<.01$, Hazard ratio $=.551,95 \% \mathrm{Cl}=.367-.829$, resp. Wald $(1)=11.134, p<.001$, Hazard ratio $=3.133,95 \% \mathrm{Cl}=1.602-6.129)$.

Overall these results suggest that time to dropout is dependent on the quality of the therapeutic alliance as perceived by patient or therapist as early as three months after the start of therapy over and above the effect of pretreatment BPDSI-scores.

\section{Zero-Order Correlations for Early- to Midtreatment and Mid- to Late Treatment Change Scores}

For each process (WAI-P, WAI-T, and DDPRQ) and outcome variable (BPDSI), early treatment scores were regressed on midtreatment scores, and midtreatment scores were regressed on late treatment scores to form early- to midtreatment and mid- to late treatment residualized chance scores. As in previous studies of process and outcome changes in panel designs (Burns, Kubilus, et al., 2003; Evon \& Burns, 2004) changes among the process and outcome variables at different time periods (i.e. autocorrelations) were non-significant, suggesting that early-treatment changes with respect to these factors were unrelated to their corresponding late treatment changes. ynchronous correlations showed that changes in process and outcome factors during the same time period were also in many cases non-significantly related, with only one significant 
association of early- to midtreatment changes in DDPRO scores with early- to midtreatment changes in BPDSI scores, $(r(57)=.31, p<.05)$. These results suggest that variations due to autocorrelation or synchronous correlations will not substantially affect cross-lagged associations.

Cross-lagged correlations demonstrated that early to mid WAI-P was significantly related to mid to late BPDSI $(r(78)=-.34, p<.01)$, whereas early to mid BPDSI was not significantly related to mid to late WAI-P $(r(78)=.11$, ns). On the other hand, early to mid WAI-T and DDPRO were not related to mid to late BPDSI, whereas the converse correlations were also non-significant.

\section{Testing Cross-Lagged Associations With Hierarchical Multiple Regressions}

Hierarchical regressions were performed to analyse whether early- to mid WAI-P change scores remained a significant predictor of mid- to late treatment BPDSI change scores when variance because of early- to midtreatment changes on the BPDSI and mid- to late treatment changes on the WAI-P were controlled (Finkel, 1995). For mid- to late-BPDSI, pre- to mid-WAI-P did emerge as a significant predictor also after controlling for the other change scores and treatment condition ( $F$ change $(1,73)=11,701, p<.001$ ). In testing the converse lagged association, no significant associations were observed (see Table 4).

Table 4. Summary of Hierarchical Regression Analyses: Cross-Lagged Regressions for Working Alliance Inventory - Patient Version $(n=78)$

\begin{tabular}{lllll}
\hline Variable & B & SE B & $R^{2}$ & $\Delta R^{2}$ of step \\
\hline & Mid to late BPDSI & \\
\cline { 2 - 4 }
\end{tabular}

Step 1

Condition

Early to mid BPDSI

Mid to late WAI-P

Step 2

Early to mid WAI-P

Step 1

$\begin{array}{lll}\text { Condition } & -.080 & .231 \\ \text { Early to mid WAI-P } & -.166 & .125 \\ \text { Mid to late BPDSI } & -.228 & .122 \\ \quad 2 & & \\ \text { Early to mid BPDSI } & .060 & .116\end{array}$

Step 2
.217

.109

.107

\begin{tabular}{lrll}
-.201 & .107 & & \\
& & 044 & .044 \\
-.376 & .110 & .176 & $.132^{* *}$ \\
\hline \multicolumn{2}{l}{ Mid to late WAI-P } \\
\hline
\end{tabular}

\section{1}

116
.062

Note. Variables are residualized change scores. Early to mid=early- to mid- treatment; Mid to late $=$ mid- to late treatment; BPDSI $=$ Borderline Personality Disorder Severity Index; WAI$\mathrm{P}=$ Working Alliance Inventory- Patient Version

${ }^{*} p<.05 .{ }^{* *} p<.01$ 


\section{Relationship of Patient-Therapist Dissimilarity to Changes on Process and Out- come Variables}

Using t-tests for independent groups, no significant differences in YSQ and IPO global scores between therapists $(n=17)$ from the SFT condition and TFP condition ( $n=13$ ) were found. Also, $D \ni^{2} \mathrm{YSO}$ and IPO dissimilarity scores of therapist-patient dyads in SFT $(n=35)$ did not differ from those in the TFP condition $(n=22$ ) (all $p$-values $>.1$ ).

Descriptively, therapist-patient $\mathrm{YSQ}$ dissimilarity scores were found to range from 15.6 to $309.0(\mathrm{M}=55.9 ; \mathrm{Sd}=47.3)$ and IPO dissimilarity scores from 7.5 to $69.9(M=23.1$; $S D=14.3)$. Because of positive skewness both dissimilarity measures were log transformed for further statistical analyses resulting in a quasi-normal distribution with adequate skewness $(<1)$.

First, it was investigated whether $D \ni^{2} \mathrm{YSO}$ or IPO dissimilarity scores predicted early- to mid changes on the WAI and DDPRO in addition to and independent of treatment condition and the absolute levels of endorsement of early maladaptive schema's by patients or the patients' personality organization. Using multiple regression analyses with patients' total YSQ scores and condition forced into the equation in the first step, YSQ dissimilarity scores had a positive and significant semipartial correlation $(\mathrm{r}(57)=.30, p<.05)$, with early- to mid-WAI-P scores (F change $(1,53)=5.33, p<.05)$. IPO dissimilarity scores also had a positive and significant correlation $(r(57)=.28, p<.05)$, with early to mid-WAI-P (F change $(1,53)=4.69, p<.05)$. The associations of YSO and IPO dissimilarity scores with early to mid changes on the WAI-T and DDPRO were all nonsignificant (all $p$-values $>.1$ ).

Next, evidence for a direct effect of therapist-patient dissimilarity on earlyto midtreatment and mid- to late treatment changes on outcome measures was investigated. None of the simple regression coefficients of $D \ni^{2}$ YSO or IPO dissimilarity on early- to midtreatment and mid- to late-BPDSI scores proved to be significant. T-tests for independent groups also failed to show any significant differences between dropouts $(n=19)$ and treatment completers $(n=38)$ with respect to YSQ and IPO dissimilarity scores $(p>.1)$.

These results indicate that a higher degree of dissimilarity in maladaptive schema's or personality organization between therapists and patients is associated with the development of a better therapeutic alliance from the patient's and not the therapist's point of view irrespective of treatment condition or the patients' absolute endorsement of maladaptive schema's or global level of personality organization.

\section{Completers analyses}

It was investigated whether the results of the analyses in the completers sample $(n=53)$ were comparable to those in the intention-to-treat sample 
$(n=78)$. The results of the analyses of early- to mid- to late treatment changes, cross-lagged associations of changes on process and outcome measures and the influence of patient-therapist similarity to changes on process and outcome variables essentially yielded the same results.

\section{Discussion}

This study had four aims. Firstly, it was hypothesized that the quality of the therapeutic alliance would be rated higher in SFT than in TFP. This hypothesis was supported both with respect to patients' and therapists' ratings of the therapeutic alliance. Furthermore, it was observed that the quality of the therapeutic alliance as rated by the patient increased in the course of treatment irrespective of treatment condition, while therapist frustration decreased in SFT but increased in TFP. These results indicate that the rating of the alliance reflecting the overall quality of experiences and feelings during a large number of therapy sessions clearly differs between treatment conditions. The higher ratings in SFT possibly reflect the effort in SFT to connect to the patient by adapting an unthreatening and supportive attitude and to develop mutual trust and positive regard (Beck, Freeman, et al., 1990; Beck, Freeman, et al., 2004; Young, Klosko, et al., 2003). Using a schema mode model might help to increase sympathy with the BPD patient, as most dysfunctional behaviors are understood as stemming from unfortunate early life experiences (Young, Klosko, et al., 2003). In contrast, TFP with a contract phase that by its working out might introduce an unnecessarily defensive and adversial tone to the therapy and in which (negative) transference manifestations are interpreted without the use of explicit supportive interventions possibly pressurize the therapeutic alliance (Gunderson, 2000) and even results in growing therapist frustration in the course of therapy.

Secondly, it was expected that a lower quality of the therapeutic alliance at early treatment would predict premature treatment termination and outcome. This expectation was partly corroborated as available therapists' ratings of the alliance were associated with early dropout in TFP during the first three months of treatment and both patients' and therapists' ratings of the therapeutic alliance after three months of treatment were found to predict time to dropout in the remaining treatment period in TFP and SFT. These results support the contention that compared to TFP the therapeutic alliance in SFT may not only be different, but also more therapeutic at least in the first phase of therapy. The finding that compared to SFT significantly more patients terminated TFP prematurely (Giesen-Bloo, van Dyck, et al., 2006) may partly be accounted for by the quality of the therapeutic alliance. As has also been stressed by Linehan and colleagues part of the dropout rate arises from the effect on the therapist of working with 
difficult patients. Both therapist and patient distress possibly can be reduced by shifting therapist interpretations of patient behavior from hostile to friendly (Shearin \& Linehan, 1992). Possibly, the first stages of TFP in which fragmented and partial aggressive self and object representations are activated and interpreted by the therapist poses too high demands on the beginning therapeutic alliance for a substantial proportion of patients with a borderline personality disorder.

Thirdly, it was hypothesized that growth of the therapeutic alliance during the first year of therapy represents an important therapeutic mechanism by which a later reduction of borderline personality disorder pathology is facilitated. The study results corroborated this hypothesis with respect to an enhanced quality of the therapeutic alliance as experienced by the patient. This result enlarges our understanding of the causal role of the therapeutic alliance in the treatment of different psychiatric disorders. Some previous studies investigating relationships among alliance and outcome changes in different periods of therapy seem to suggest that growth of the alliance may be a mere epiphenomenon of treatment gain (e.g. Evon \& Burns, 2004; Tang \& DeRubeis, 1999). However, it is conceivable that in structured, manualized and short-term treatments of many Axis I disorders a collaborative therapeutic relationship is readily accomplished with relatively few complications masking the fact that such a relationship is a necessary precondition to treatment. Of note is that in most treatment studies in Axis I disorders ratings of the quality of the alliance with the WAI are generally high possibly restricting associations with other variables because of restriction of range. Although the somewhat different answering format of the WAI in the present study precludes a direct comparison with previous studies, the ratings of the alliance in the present study in patients with borderline personality disorder, although positive, seem somewhat lower possibly allowing finding relationships with other variables, such as treatment condition, premature termination and subsequent improvement. In the more semi-structured and long-term treatment of Axis II disorders the development and maintenance of the therapeutic alliance constitutes a central issue of therapy and may constitute a central curing mechanism (Orlinsky, Ronnestad, et al., 2004). Consequently attaining this goal will affect therapy outcome. It seems worthwhile to continue research into the intertwined and sequential relationships between alliance and client's improvement during treatment in patient samples where the establishment of the therapeutic alliance is not always readily accomplished (such as Axis II disorders but also Axis I disorders like addictive behavior or medically unexplained somatic symptoms).

The last aim of this study was to test whether dissimilarity in pathological personality characteristics between therapists and patients would facilitate the development of the therapeutic alliance and indirectly affect therapy outcome. 
Although dissimilarity in pathological personality characteristics directly influenced the growth of the therapeutic alliance as rated by the patient, it showed no relationship with outcome.

These results show that it is fruitful to study the impact of therapists' variables in a relational or interpersonal context (Beutler, Malik, et al., 2004) and also that differentiating between (in)direct effects on process or outcome can yield a more balanced view of the causal network in which the alliance-outcome link is embedded (cp. Hilliard, Henry, \& Strupp, 2000). The present findings are in accordance with the presupposition of clinicians from various theoretical orientations that therapists must be able to preserve a neutral part of their mind that is able to accurately monitor and analyze their reactions provoked by schema activation or projective identification.

Taken together, our study results seem to suggest that the therapeutic alliance constitutes an important common factor in the psychotherapeutic change process partly influenced by therapist-patient dissimilarity in personality profile. However, this does not imply that the therapeutic alliance is a "necessary and sufficient" component of change in the treatment of borderline personality disorder. Clear alliance differences between treatments indicate that the quality of the alliance is affected by the nature of treatment. Apparently factors specific to a particular approach influence the quality of the alliance and SFT with its emphasis on the "necessary and sufficient conditions" as identified by the clientcentered school produces a better alliance according to the ratings of both therapists and patients. In addition, the magnitude of the consistent and positive association of alliance with outcome is relatively modest as in most previous studies (Martin, Garske, et al., 2000; Orlinsky, Ronnestad, et al., 2004). It is extremely unlikely that any single process or mechanism will adequately explain most of the variance in outcome. So, the causal role of the alliance does not rule out the possible role of other common factors (such as mitigation of isolation) or variables unique for a particular treatment approach (such as developing more healthy schemas or enhancing reality orientation).

There are at least two reasons to think that the current data deserve serious considerations. First, we used a DMS-IV BPD criteria based semi-structured interview with good psychometric properties as primary outcome measure instead of relying on a self-report measure (Arntz, van den Hoorn, et al., 2003; Giesen-Bloo, Wachters, et al., 2006). Secondly, data on the quality and development of the therapeutic alliance were collected in the course of a randomized clinical trial of intensive long term SFT and TFP in a rather large and clinically representative group of BPD patients (Giesen-Bloo, van Dyck, et al., 2006).

At least three limitations of this study merit consideration. First, the 12 and 18 months treatment lags probably are too extended to accurately detect the timing of process and outcome changes. Collapsing across a 12 month treat- 
ment period and then trying to relate process and outcome changes to changes collapsed across the following 18 months of treatment may have diluted or obscured the actual effects of the therapeutic alliance on outcome or vice versa. In future research it may be advisable to adapt shorter time lags in order to analyze the precise sequence of process and outcome changes (Orlinsky, Ronnestad, et al., 2004; Tang \& DeRubeis, 1999). Secondly, also the results of cross-lagged panel design analyses remain correlational and preclude definitive conclusions about the direction of causality (Finkel, 1995). Thirdly, because eight of the 86 patients included in the randomized controlled trial prematurely terminated TFP treatment between pre-treatment and the first assessment of the therapeutic relationship after three months, the significantly greater efficacy of SFT compared to TFP (Giesen-Bloo, van Dyck, et al., 2006) could not be demonstrated in the present study in 78 patients because of reduced statistical power and noninclusion of these eight non-responders all from the TFP condition. Consequently, no complete mediational analysis (Baron \& Kenny, 1986) could be performed in order to investigate whether the superior efficacy of SFT is mediated by differences in the quality of the therapeutic relationship between treatment conditions.

In sum, results provide support for the assertion that type of treatment differentially affects the quality and development of the therapeutic alliance and that dropout rate and clinical outcome can be partly accounted for by the quality of the therapeutic alliance. Especially ratings of the therapeutic alliance by therapists at early treatment are predictive of dropout, while growth of the therapeutic alliance as experienced by patients in the first part of therapy seems to predict subsequent symptom reduction. These results suggest that the therapeutic alliance is one of the factors producing change in the treatment of BPD patients and that adapting the type of treatment may be an important avenue in trying to enhance the quality of the therapeutic alliance and clinical outcome. 


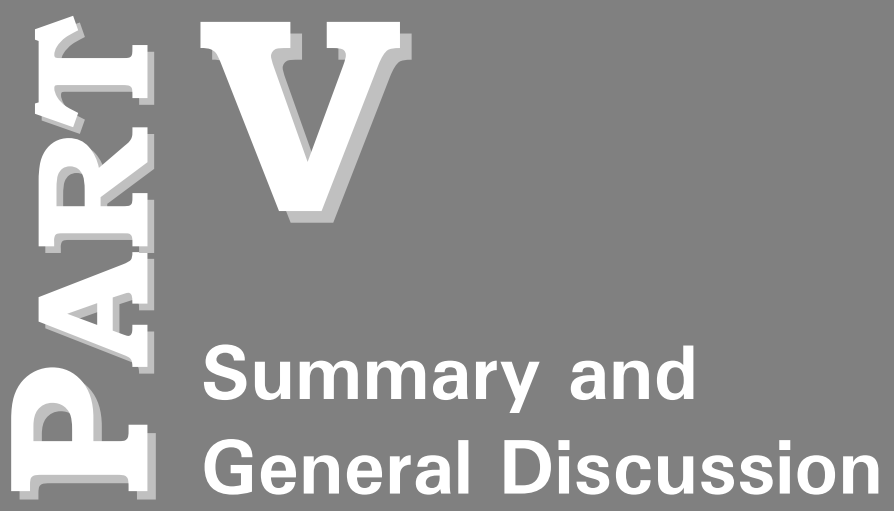





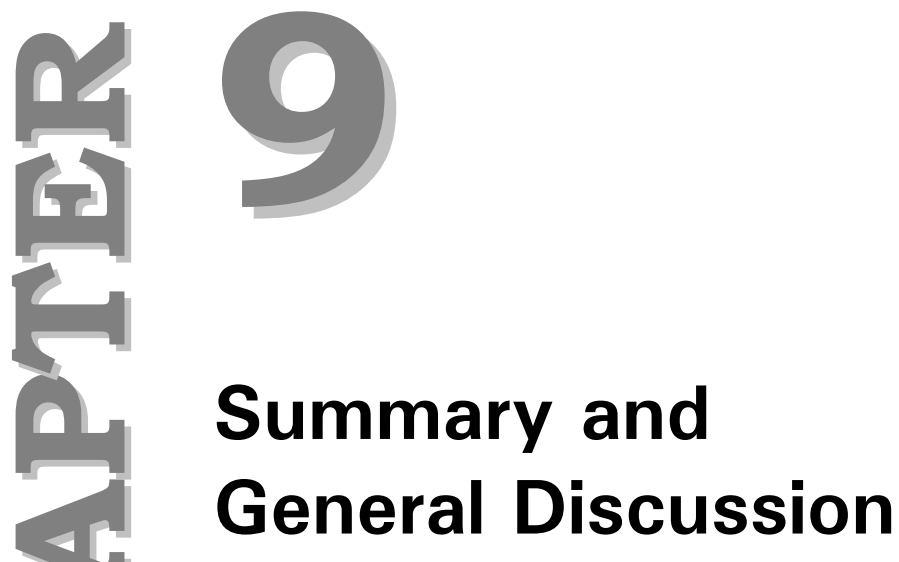





\section{Summary and General discussion}

This thesis started with an overview of the current state of affairs in our knowledge of borderline personality disorder (BPD) with respect to its conceptualization, patient characteristics, etiology, assessment and (experimental) treatments. Within these domains, different empirical research issues were defined and tested in the studies presented. This chapter will give a summary of the findings, followed by a general discussion; including implications for clinical practice, as well as directions for future research.

\section{Summary of study conclusions}

Part II Chapter 2 It was tested whether BPD is characterized by specific world views as hypothesized by cognitive models, using Janoff-Bulman (1989) world assumptive model of negative effects of trauma. A second aim of this study was to investigate the role of trauma in the content of world views of BPD patients. The cognitive theory's central assumptions for BPD (the world is (i.e. others are) dangerous and malevolent; I am powerless and vulnerable; and I am inherently unacceptable) were to a large extent supported by using JanoffBulman's theoretical framework of world assumptions (Janoff-Bulman, 1989). The content of world assumptions of BPD-patients could better be explained by BPD-specific pathology than by presence and severity of childhood trauma.

Chapter 3 In this chapter the psychometric properties of the 48-item Defense Style Questionnaire (DSQ-48) were investigated. Other aims of the DSQ48 study were to replicate the three-factor structure of the DSQ-36 (Spinhoven, van Gaalen, et al., 1995) and to compare the defensive style of different patient and non-patient groups. With respect to the presence and role of defense mechanisms, as defined by the psychoanalytical object-relation theory, BPDpatients can indeed be characterized by the use of primitive defense mechanisms as assessed with the DSO-48 (and the IPO-borderline organization structure), and can be distinguished from other patient and non-patient groups. The psychometric evaluation revealed some shortcomings, eventually leading to an alternative 5-factor structure (distrust, self-protection, self-sacrifice, avoidance and control) with better psychometric properties. Forty-three of the 48 DSQ-items were included.

Part III Chapter 4 and 5 The studies in these chapters examined the assessment and conceptualization of borderline personality disorder with the 'BPDSI-IV' semi-structured interview and the Borderline Personality Disorder Checklist (BPD Checklist) self-report questionnaire. The BPDSI-IV assesses the severity and frequency of BPD-pathology in detail, the BPD-Checklist assesses 
the burden experienced from BPD-pathology. Both instruments proved to be psychometrically sound by having good internal consistencies, and good discriminant, concurrent and construct validity. Clinical norms, specificity and sensitivity were derived for clinical and research applications. The BPD Checklist further revealed sensitivity to change, and the same applied to the BPDSI-IV which was most specifically shown in Chapter 6 . The second research aim involving the BPD Checklist and BPDSI-IV led to the conclusion that, by using the items of either instruments, both a one- and a nine dimensional model based on DSM-IV BPD-criteria have empirical validity. The validity of these one- and nine factor dimensional models was better than of three- or four higher order factor models as suggested in previous research.

Part IV Chapter 6 and 7 The effectiveness of three years of Schema Focused Therapy (SFT) and Transference Focused Psychotherapy (TFP) was investigated in a multicenter randomized controlled two group design. Maintenance of the results was examined after a 1-year follow-up period. Three years of twice weekly SFT and TFP proved to be effective in reducing borderline specific psychopathology, general psychopathology and measures of SFT/TFP concepts, and in improving quality of life. After three years, SFT was more effective than TFP on all measures. One-year follow-up results continue to show a positive treatment perspective for BPD, as more BPD-patients achieved recovery and/or reliably changed status than after 3 years. At year 4 , SFT remained favorable over TFP with regard to reduction in BPD-pathology, general psychopathology and measures of SFT/TFP concepts. SFT and TFP were equal effective for quality of life at 1 -year follow-up.

Chapter 8 The quality and development of the therapeutic alliance as a mediator of change in SFT and TFP was studied. Therapeutic alliance scores were higher in SFT than in TFP. Negative ratings of therapists and patients at early treatment were predictive of dropout, while increasingly positive ratings of patients in the first half of treatment predicted subsequent symptom reduction. Dissimilarity between therapist and patients in pathological personality characteristics had a direct effect on the growth of the therapeutic alliance, but was unrelated to outcome. Based on these findings, it was concluded that the therapeutic alliance is an important common therapy factor critically affected by type of treatment.

Overall it can be concluded that borders have been crossed in borderline personality disorder research. A first border concerned testing (parts of) two psychological models of BPD by using different, cognate psychological theories; Beck's cognitive BPD core assumptions were tested by Janoff-Bulman's social cognitive world assumptions; defensive styles were tested against object-relation 
based primitive defenses and cognitive based coping mechanisms. A second border involved the development of reliable, valid and change-sensitive assessment instruments (the BPDSI-IV and the BPD Checklist), specifically designed for BPD-pathology in screening- and treatment outcome procedures. This includes the different self-report and semi-structured interview formats, even enabling both subjective and objective BPD-pathology assessments. A third border crossed is the successful enterprise of executing a prolonged well-designed randomized clinical multicenter trial of outpatient treatments for borderline patients (Schema Focused Therapy versus Transference Focused Therapy). Moreover, this randomized trial (RT) empirically supports a changing perspective on BPDtreatment: from untreatable to treatable. Finally, the fourth border passed, relates to the unique collaboration of two different psychological treatment formats in one trial.

\section{Discussion of the borderline treatment study}

Many psychological theories and (manual-based) treatments have been developed over the last two decades (see chapters 5 and 6 for references). As a result, the opinions on the possibilities of treating BPD have fortunately become more optimistic. However, research to provide an empirical basis for BPDspecific theories and treatments is still lagging behind. A major aim of this thesis was to contribute to the empirical knowledge by evaluating two relatively new outpatient BPD-treatments. This paragraph will discuss the methodological strengths and weaknesses.

A first strength of this Borderline treatment study is that all participants were screened with optimal diagnostic procedures (Zimmerman, 1994; Zimmerman \& Mattia, 1999a; b), using DSM-IV based semi-structured clinical interviews (SCID-I and -II) that have proven to reliably and validly assess the DSM-IV Axis-I and -Il disorders (First, Gibbon, et al., 1997; First, Spitzer, et al., 1996; Groenestijn, Akkerhuis, et al., 1999; Weertman, Arntz, et al., 2000). Second, the use of a wide, 2-month time frame for the screening procedure was valuable for several referred patients, who realized during this phase that they could and/or would not be able to meet the expected time-investment in therapy and research assessments, and therefore protected both SFT and TFP from attrition unrelated to the treatment itself. However, this wide time frame has also a negative side to it, as it resulted in a selection process among the borderline patients and therefore limits generalization. Other strengths are (3) the sample size, (4) the study's duration, (5) regular 3-monthly assessments with an additional 1-year follow-up enabling to chart progress and treatment processes in detail, (6) the wide array of assessment instruments including psychometrically sound ob- 
server-rated and self-reported a-theoretical measures, theory guided instruments and process measures, (7) the participation of therapists who were trained by treatment developers dr. Young (SFT) and dr. Yeomans (TFP), (8) the use of treatment integrity procedures and (9) and the high comparability of SFT and TFP with respect to treatment intensity and treatment aim (fundamental personality change leading to full recovery).

Even though many strengths could be noted; no study is perfect, nor are study results ever totally unambiguous. As discussed in chapter 6 and 7, limitations of the present study are (1) the absence of a regular treatment-as-usual (TAU) and/or a non-treatment condition, (2) non-blindness of research assistants as the study progressed, as patients talked about their treatment and therapist and (3) non-blindness of the study psychiatrist. Besides the limitations, some observations of the BPD outcome study deserve additional attention.

A first observation is that during the study it became clear that some of the patients had comorbid disorders, which were pre-set exclusion criteria and should have been detected during the screening procedure. According to their therapists, at least three patients apparently suffered from dissociative identity disorder (DID). The current procedure included a screening step with the Dissociative Experience Scale (DES; Boon \& Draijer, 1995; Draijer \& Boon, 1993). If a potential participant had a total score above 25, the elaborate DSM-IV based interview for DID was administered (SCID-D; Boon \& Draijer, 1995). One can wonder whether the DES-cutoff score of 25 was too high, whether the two-step screening procedure was inadequate and all patients should have been interviewed, or whether the SCID-D's properties should be questioned. When balancing methodological and feasibility aspects, the chosen screening procedure on DID still can be considered optimal. However, what becomes evident from this study's experience is that not only experienced screeners are needed but that (peer-) supervision in the screening process is advisable, as well as resistance to time-pressure (e.g. in waiting for old hospital records). As for the current study, two DID-patients were fortunately randomized to SFT, one to TFP, and this allocation more or less reduced a possible unequal influence on treatment outcome. Two other patients prematurely stopped treatment due to psychotic decompensation. Independent retrospective file-based diagnostic procedures learned that one patient was evidently wrongly included, as an earlier psychotic episode was overlooked. The other patient probably suffered from an organic psychosis. Both were allocated to the SFT condition, implying that their inclusion probably counteracted the efficacy of SFT. Still, all patients were included in the outcome analyses for two reasons. Firstly, it was pre-determined that conservative statistical procedures would be employed in order to avoid any artifacts, which means that all available 'cases' should be included. The second argument refers to the intention to study BPD-patients who are optimally representative for a regular 
clinical set of BPD-patients. It was reasoned that wrongful or missed diagnoses are also part of real-life in mental health care. Thus, although it was highly undesirable for both SFT/TFP outcome results to include "false positives", the interest of external validity overruled post-hoc considerations.

A second observation concerns three patients who fell out the study during the TFP-contract phase as patient and therapist were unable to start the contract phase or reach a (verbal) contract. One can ask whether these BPD-patients should be viewed as drop-out due to unsuccessful treatment, since this prephase determines the framework to start actual TFP. In the view of the developers of TFP, a drop-out patient in the contract phase is protected from an ineffective (and unwanted) treatment endeavor. The contract phase aims to function as a filter, and assists optimal treatment indication. However, empirically speaking, including a statistical correction for this functional TFP-feature did not seem warranted as an exclusion from treatment in the contract phase is also an outcome. Another argument is that several studies report on attrition rates as treatment outcome in themselves (Bateman \& Fonagy, 1999; Linehan, Armstrong, et al., 1991), mostly because keeping borderline patients in any treatment has for long been a primary objective in itself. Therefore, reasons of drop-out (including contract phase drop-outs) are separately reported to make individual conclusions possible (Chapter 6, Figure 1).

A third remarkable observation concerns the fact that most TFP dropouts occurred during the first treatment year, whereas most SFT dropouts were counted in the study's second 1.5 year. It was a priori hypothesized that dropouts, if any, would happen during the first year of treatment. This was based on the instability in (meaningful) relationships that many BPD-patients experience. Starting treatment for a borderline patient implies the difficult 'task' of starting a new meaningful relationship with a therapist, exposing oneself to potential risk for additional problematic experiences. This probably also explains (part of) the TFP-dropout occurrence. The differences in therapeutic role between TFP and SFT (dynamically neutral, reflective versus supportive, directive) could also be of influence. From a clinical prospect, late non-responding drop outs are most inconvenient, one wants to detect non-responsiveness (dropout) as soon as possible, 'to help as many patients with less money in less time'. The late SFT dropout patients did not significantly improve on the primary outcome measures. In further developing SFT, specific attention should be paid to characteristics involving SFT-indication. Treatment progress monitoring can also contribute in detecting (and acting) on non-responsiveness, as studies have shown that providing feedback to therapist and patient on the patient's progress can enhance treatment outcome (Lambert, Hansen, \& Finch, 2001; Lutz, Rafaeli, Howard, et al., 2002). When examining the study events and results in retrospect, one should not only search for explanatory factors in different treatment characteris- 
tics but also in (intertwined) therapist and patient characteristics. Paragraph 9.3 will focus on some of these characteristics.

A fourth issue of the borderline treatment study to be addressed regards the ongoing development of the SFT and TFP manuals during the study. Even though both treatment manuals appeared to be at an advanced, final state when the study started, some changes in the guidelines of both SFT and TFP were observed at national supervisions by the treatment developers dr. Young (SFT) and dr. Yeomans (TFP) over the years. An example for SFT is, what was initially called '24-hour reachability' (by telephone) of the therapist, requiring that a SFTtherapist should also be reachable to the patient outside office hours at any time. At first, this seemed a quite strict prerequisite for a therapist to participate in the treatment study. However, the clarification (and application in the Dutch mental health care tradition) of this issue took some time but made clear that a SFTtherapist has room to set personal limits with respect to availability to a patient outside office hours. For instance, a therapist can explain to the patient why he/she cannot answer the phone and discuss what in that case has to be the next step for the patient because he/she is away, on a break, asleep, etcetera. An example for TFP concerns the guidelines for deviating from a technical neutral stance. At first, active involvement of the TFP-therapist towards the patient outside therapy sessions was only very rarely allowed and if permitted, would often lead to termination of TFP and was considered as a (serious) contract breech by the patient. Later in the study, the TFP-therapist appeared to be allowed to temporarily deviate from, and then return to, neutrality more easily without necessarily losing or breaking with the TFP-treatment frame. These developments were completely natural, understandable and justifiable from a clinical perspective. However, from an efficacy research perspective these developments at that time were not welcome since a researcher wants to have clearly defined, stable interventions. Therefore, it should again be emphasized that the outcome results need balanced interpretation. The SFT- and TFP-manual in a next RT will undoubtedly be somewhat different from the SFT- and TFP-manual of the present RT.

The study results present a positive message by increasing the evidencebased treatment possibilities for BPD-patients. However, in studying long-term treatments outcome, the focus is not only effectiveness in itself, but also the relation between treatment duration and effectiveness. Justification of long-term therapy is an issue with considerable clinical (i.e. therapists case loads, waiting lists, achieving optimal improvement) and economical (i.e. health care insurance policies, governmental regulations and mental health care budget) implications. For BPD-patients it is already argued (introductory chapter) that short-term treatments are not sufficient and long-term treatments are indicated. With respect to SFT and TFP, observing the improvement patterns of successful pa- 
tients, as well as identifying factors that were predictive of patient-dropout, can help to define criteria that optimize indication and justification of long-term treatment. Differences between SFT and TFP can be incorporated in these criteria, and may also contribute to a higher and more efficient treatment success/effectiveness rate for both treatments. Unfortunately, Dutch political decision-making in mental health care has a tendency to act before relevant results are available. As a result, many BPD-patients today are denied optimal recovery opportunities and what's more from a societal perspective, savings are missed (e.g. through reduced use of Disablement Insurance Act, Dutch Health Law, unemployment). This study demonstrates the short-sightedness of recent governmental policy changes with respect to the limitations on reimbursement of individual psychotherapy. The now imposed maximum of 50 therapy sessions for BPD patients is absolutely insufficient to reach recovery and/or significant improvement.

In addition to the current discussion, it must be noted that another major part of this study was assessing TFP and SFT's cost-effectiveness (van Asselt, 2006). Preliminary analyses reveal that psychotherapy (either SFT or TFP) from an economically based 'quality adjusted life year'-unit (QALY) point of view, already becomes cost-effective during treatment. In other words, although society has to invest in individual psychotherapeutic treatment, already during treatment society will receive more money back than invested, while quality of life of the patient increases. Cost-effectiveness calculation of SFT and TFP will be available soon. Other issues in BPD that were investigated with data obtained within the present trial centered around three foci: (1) relations between autobiographical memories, memory processing and childhood trauma (Kremers, 2004), and (2) relations between informational processing and cognitive and psychodynamic theories of personality disorders (Sieswerda, 2006). These (sub-) studies further illustrate the significant contribution of the present study framework to an empirical basis for understanding and treating BPD.

\section{Patient - therapist factors in treatment outcome}

With respect to patient - therapist factors, a general distinction can be made between factual variables (e.g. age, gender and other numbers) and process variables. Process variables appear more fluid and often contain an interactive component between patient and therapist, in which the 'factual' factors also play a role (e.g. patient-therapist relationship; therapists' capacity to endure aggression; patients' capacity to self-reflection). As said, the borderline treatment study also investigated determinants of the therapeutic alliance and treatment outcome (See Chapter 8). 
Most treatment outcome studies ascribe treatment effects in patients to the investigated treatment(s), meaning the specific systematic appliance of theoretically defined treatment techniques and strategies. The influence of the relationship between patient and therapist, the therapeutic or working alliance, is also often acknowledged and considered important, see for instance Chapter 8 . Many psychotherapeutic treatment protocols from different backgrounds (like SFT and TFP), emphasize the importance of the therapeutic alliance for optimal treatment results and include, in addition to technical and strategic aspects, specific directions to the interpersonal style a therapist should adopt in treatment. Treatment integrity checks, including the therapeutic stance, are often tailor-made to one treatment, making comparisons between therapeutic stances from different treatment perspectives problematic. Most studies focusing on the patienttherapist relationship in relation to psychotherapy outcome and drop-out, often do not report specifically on the contribution of therapist background to the patient-therapist relationship, and with that more or less implicitly assume that the therapeutic interpersonal style is not substantially different across theoretical schools (Kiesler \& Watkins, 1989; Wallner Samstag, Batchelder, Muran, et al., 1998). Along the ratings for the treatment integrity procedures (Chapter 6) and therapeutic alliance ratings (Chapter 8 ), an exploration whether this implicit assumption is accurate was undertaken. Results of this pilot study can be found in Appendix I. Chapter 8 and the pilot study show, in contrast to the referenced literature, that therapeutic alliance and therapists' interpersonal style are not aspecific, and appear related to treatment method and therefore to therapist background / theoretical schools. Moreover, alliance ratings were predictive of drop-out and treatment outcome. And although the reliability of the pilot study cannot be warranted, it is still striking that the lower affiliation scores of TFPtherapists on the Interpersonal Behavior Observation (IBO) compared to SFTtherapists are in line with the more negative alliance ratings of TFP-therapist than those of SFT-therapists. The present findings further demonstrate that treatment outcome is indeed empirically determined by both treatment method and patienttherapist relationship (including the contribution of therapist's interpersonal style). However, it will be a complicated task to clarify in future studies how and to what extent therapeutic alliance, and inherent to that, therapist's interpersonal style mediate and/or moderate treatment outcome.

The SFT- and TFP-therapist groups were similar in professional status, experience, age and personality features (Young Schema Questionnaire and the Inventory of Personality Organisation). Furthermore, gender did not prove to have a significant contribution to outcome. Still, one should realize that these factors are of influence, in most cases indirectly, on treatment outcome. For example, it will cost a relatively inexperienced therapist probably more attention and effort to adequately set limits during therapy than one of the study therapists. And it 
was already observed during the study that some therapists also had difficulties with limit setting (either in SFT or TFP, and either due to individual character or treatment model interpretation). This can, in turn, increase treatment duration. It is of importance that these aspects are addressed in treatment supervision in order to ensure an optimal match of therapist and patient. An optimal match in personality dimensions was found to be of positive influence on the therapeutic alliance. However, the subsequent effect of this match on treatment outcome is negligible.

Some instability in the SFT therapist group was observed during the study period, which of course was unwanted from an empirical perspective but realistic for the clinical practice of personality disorders (thus enhancing external validity of the SFT-results). Changes in therapist availability can in part be explained by the long duration of the study. However, all switches of therapists within a treatment process in the 3-year study period remarkably happened in the SFTgroup. Four SFT-patients had to switch therapists for a four month period as their own, initial therapist was on maternity leave. Another four SFT-patients had to switch due to changes in their therapists' personal life (e.g. job change, longterm sick leave). Still, how (or if) these switches have affected SFT outcome, cannot be determined, as $n=8$ is too small for a reliable assessment.

A noteworthy patient related factor was the observation that treatment outcome was negatively influenced by use of psychotropic medication. In achieving optimal treatment indication for patients, one can wonder how use of psychotropic medication should influence the decision to offer SFT or TFP. It is no option to deny BPD-patients on psychotropic medication psychotherapeutic treatments; a substantial part of the BPD-population would then be deprived of treatment while these patients do improve, but to a lesser extent than those without psychotropic medication. Furthermore, it is not unlikely that BPDpatients on psychotropic medication form an a priori negative selection. However, it could be useful to investigate whether this subgroup of BPD-patients might benefit more from other (manual-based) BPD-treatments, especially if such a treatment would turn out to be as effective but less intensive and/or less costly. Another hypothesis to be examined, following the present observation and Simpson et al 's finding (2004) that single dialectical behavioral therapy (DBT) was more effective than a combination of DBT with psychotropic medication, is that treatment outcome could improve when potential SFT- (and TFP-) patients would stop or reduce psychotropic medication as much as possible before entering or during treatment. Furthermore, it is of interest to determine if different types of psychotropic medication (antidepressants versus anxiolytics versus antipsychotics) have differential effects on psychosocial treatment outcome. 


\section{Categorical and dimensional conceptualization}

The debate on dimensional versus categorical conceptualizations of personality disorders is rather intense, as demonstrated by the large literature on this subject (e.g. Costa Jr. \& McCrae, 1990; 1992; Frances, 1982; Livesley, Jang, \& Vernon, 1998; Nelson-Gray \& Farmer, 1999; Watson, Clark, \& Harkness, 1994; Westen, ; Widiger \& Mullins-Sweat, 2005). All patient classifications used in this thesis are based on the DSM-IV categorical approach of BPD. The DSM-IV-TR (APA, 2000) refers to the dimensional perspective as an alternative for the categorical model in which "personality disorders represent maladaptive variants of personality traits that merge imperceptibly into normality and into one another" (p.689). Most objections to the categorical conceptualization of personality disorders concern excessive diagnostic co-occurrence, heterogeneity among persons with the same diagnosis, absence of a non-arbitrary boundary with normal functioning, and inadequate coverage of maladaptive personality functioning (e.g. Widiger \& Mullins-Sweat, 2005). Heterogeneity as demonstrated by comorbid disorders, was indeed observed in patient groups from different studies (chapters 2-8). Especially the personality disordered patients had substantial comorbid diagnoses that at times interfered with preset exclusion criteria. For example, patients qualifying for the Cluster $C$ group were not allowed to have more than two traits of a Cluster B personality disorder (SCID-II). Several potential participants were excluded because of this, somewhat reducing the overall clinical representativeness of the Cluster $\mathrm{C}$ group patients.

Despite the disadvantages, there are theoretical and practical aspects that argue for a categorical model. Medicine has always approached illness descriptively and categorically. Only when personality disorders were noted and accepted as a group of mental disorders, the history of personality disorders as such started, and empirical research in this field was initiated (Paris, 1996). The literature on dimensional views of personality proves that these may theoretically be well-founded and may form an optimal base for studying regular/normal/healthy personality but that the dimensional models' relations with psychopathology is yet unclear (Watson, Clark, et al., 1994). Focusing on the present clinical applications of a dimensional perspective, especially with respect to (borderline) personality pathology, problems arise. When having personality functioning represented by a single dimension, the boundary between adaptive and maladaptive personality functioning may be more accurate and complete than in a categorical classification system, but this can easily become cumbersome and impractical in clinical settings. Unlike axis-I disorders, BPD (and other PD's) cannot be shortly described. Categorical constructs (diagnostic labels) seem more accommodating and easier to use, with only one central connotation 
for all constructs/personality disorders to keep in mind: differences between individuals with the same diagnosis do exist. The widespread use throughout medical and mental health care further illustrates the practical strength of categorical data. The issue of individual differences is recognized as much research within the categorical framework on the identification of (higher-order) factors within diagnoses is performed.

Two of the studies (chapter 2 and chapter 3) tested possible categorical classifications within a BPD diagnosis. The similar designs of these studies only varied in the instruments by which different DSM-based categorical BPD-models were tested, which enables a highly valid comparison of the results. Both studies support the present DSM-IV BPD-concept with identical findings. Either data could be best interpreted with a one- or nine factor structure, as interpretations of three or four higher-order factors led to considerable information loss. These results argue against the 'dimensional' objection of heterogeneity as being a diagnostic limitation; individual differences still come together in one underlying perspective. On the other hand, one can view the nine separate DSM-IV criteria as a dimensional description of BPD. Adding either BPD-Checklist or BPDSI-IV to a regular categorical screening or diagnostic procedure, would be even more accommodating towards the dimensional approach, since that would chart a larger heterogeneity-part with additional unique information.

So far, the DSM-model has been largely used to illustrate the contrast of categorical versus dimensional conceptualization with regard to personality disorders. Another line of categorical classifications that are related to personality disorders finds its origin in different psychological orientations. The DSM-IV is a descriptive and a-theoretical system that, as said before, provides common ground and a common 'language' overcoming professional and theoretical differences in the clinical field. Alternatively to the DSM-IV, theory-driven classification models are likewise able to add to the dimensional - categorical debate. Relevant examples here are Beck and colleague's cognitive model of personality disorders (Beck, Freeman, et al., 1990; Beck, Freeman, et al., 2004) and Kernberg's psychodynamic model of personality organizations (Kernberg, 1984; Kernberg, 1996), of which borderline specific parts (BPD key assumptions of the cognitive model and BPD primitive defense mechanisms of the psychodynamic model) were subjected to empirical testing in chapters 2 and 3 . As for the cognitive theory on BPD, good support was obtained for the core assumptions that are believed to be specific for BPD (Beck, Freeman, et al., 1990; Beck, Freeman, et al., 2004). And within another theoretical line, the DSQ-48 was able to prove that with respect to defense mechanisms BPD-patients are indeed characterized by primitive (or immature) defense mechanisms. In addition to the theoretical implications, these findings add to the validity of the SFT and TFP treatment models that were evaluated in chapter 5 and 6 . With respect to the role of childhood 
trauma in the etiology of BPD, correlational evidence further endorsed the cognitive perspective: not the presence of trauma determined world views (or BPD assumptions) but the way it becomes part of personality (i.c. BPD). In sum, both studies were supportive of the validity of categorical theory-driven classifications for borderline personality pathology.

Another consideration is that the dimensional model currently has hardly any treatment directions available for a patient with a dysfunctional dimensional personality profile, let alone for BPD. This is in contrast with numerous and extensive treatment directions based on categorical systems, of which Schema Focused Therapy and Transference Focused Therapy are two examples. Categories apparently are also deemed necessary within the dimensional model, at least with respect to treatment implications. 'Dimensional' suggestions have been made to retain personality disorders as lower-order (behavioral) expressions of personality traits, which would facilitate clinicians to treat symptoms, in addition to an explicit dimensional formulation of (borderline) personality and while avoiding much overlap as occurs with (DSM-IV) comorbidity (e.g Widiger \& MullinsSweat, 2005). Other propositions to use dimensional as well as categorical models complementarily, or to attempt incorporating valuable features of both models in one general approach, have been made as well (Clarkin, Hull, Cantor, et al., 1993; Paris, 1996; Saulsman \& Page, 2004; Shedler \& Westen, 2004; Skodol, Siever, et al., 2002; Trull, Widiger, et al., 2003; Westen \& Shedler, 1999). Whether personality disorders should be classified categorically or dimensionally remains an empirical issue. At present, several taxometrical studies suggest a dimensional conceptualization of personality disorders over a categorical conceptualization (Ayers, 2000; Haslam, 2002; Rothschild, Cleland, Haslam, et al., 2003; Simpson, 1994; Trull, Widiger, \& Guthrie, 1990).

\section{Assessment related issues}

Three of the studies presented contain psychometric evaluations of the Defense Style Questionnaire - 48 (DSQ-48), the BPD Checklist and the Borderline Personality Disorder Severity Index-IV (BPDSI-IV). As already pointed out in the introductory chapter, there is an interface between assessment and conceptualization. The relations between these two facets can take two directions: (1) assessment leading to support or rejection of (underlying) conceptualizations and (2) conceptualizations leading to development or use of tailor-made assessment instruments.

The DSQ-48 study pre-eminently emphasizes the importance and necessity of continuous empirical evaluation in that it is possible that relations between assessment and concepts can change, or as in this case, were adjusted. In the 
present study on defense style, it was decided that the general conceptual meaning was supported, as BPD-patients could be well described within the framework, but adjustments in the classification of defense styles were suggested. Difficulties and differences in ordering defense mechanisms have been likewise encountered and reported by others, and often relate to differences in assessment methods, such as self-report, Q-sort and observer-rated instruments (Bond, Gardner, et al., 1983; Perry \& Cooper, 1989; Vaillant, 1976). Even though it is one of the few aspects within the psychoanalytical tradition that is scientifically investigated for decades, one can wonder whether the self-report DSQ-48 is the best suitable option for testing theoretical defense issues. The self-report format probably limits assessment of defense mechanisms to a more strategic type, excluding types that are context-dependent. The clinical utility of defense mechanisms is quite clear, as these are solidly incorporated in psychodynamic oriented (manual-based) treatments like Transference Focused Psychotherapy. Still, after reviewing the literature on defense mechanisms over the last decades, it appears especially difficult to pinpoint, assess and consistently describe mature defense mechanisms in an overall theoretical and clinical concept. In that respect, immature and neurotic defense mechanisms seem more unequivocal. This impression is further supported by the results of the treatment study, in which the DSQ-48 was included as a secondary outcome measure of TFP-based personality change. The principal component analyses of all secondary measures revealed that the mature defense mechanisms scale was the only scale, of 14 scales, to behave differently (and was therefore excluded from subsequent analyses). In contrast, DSQ-immature and neurotic defense mechanisms and IPO-lower level defenses (similar to immature defense mechanisms) confirmed the hypotheses as patients' improvement and healthier daily functioning also became visible in reduced use of immature and neurotic defenses. These findings contribute to the conceptual validity of immature and neurotic defense categories in themselves. On the other hand, toning down the conceptual stability, the newly proposed factor labels (distrust, self-protection, self-sacrifice, avoidance and control) and the factors' order from most pathological to healthy, make the questionnaire appear less specific for psychoanalytical theory. The information obtained from a DSQ-43 could probably also be framed within cognitive behavioral theory, where it would refer to the use of coping strategies.

The Young Schema Questionnaire and the Personality Disorder Belief Questionnaire - BPD section were (self-report) cognitive theoretical measures of personality change in the borderline treatment study. Like the immature and neurotic defense scales, these measures confirmed the hypotheses (improvement led to reduced presence of pathogenic schemas and beliefs) and showed their conceptual validity in the cognitive framework of BPD. 
The assessment - conceptual relations of both the BPDSI-IV and the BPD Checklist with the DSM-IV BPD diagnosis presently have an excellent fit. The concurrence in BPDSI-IV and BPD Checklist score patterns for different (non-) patient groups, despite the different formats, can be viewed as further support that the current BPD-concept is well reproduced. The internal structure of the 'unstable relationships' criterion of the BPD Checklist differed somewhat from the BPDSI-IV division of this criterion in 'partner relations' and 'relations with other people', but this difference seems to play a relative minor role as both instruments yield such similar results. It will depend on the purpose and setting, in addition to other practical arguments, which instrument will be used. The applicability of these instruments is, in essence, identical. The correspondence between the BPDSI-IV and BPD-Checklist with regard to conceptualization and application purposes, and most importantly for displayed 'behavior' in different populations, opens up new possibilities. One prominent example concerns the exploration of perceptional differences of actual symptomatic change between a therapist, the patient, and an independent rater (the last one only for the BPDSIIV). This will increase empirical understanding of BPD-specific change mechanisms due to treatment, but also enables therapists to objectify his/her perceptions and hypotheses of patients' behavior. The application of assessment instruments in treatment (as 'independent' tool) can also serve as an external pathway to introduce relevant topics in the treatment process.

Clinical cutoff scores, as well as norms with accompanying specificity and sensitivity, were calculated for both total scores of the BPDSI-IV and the BPDChecklist. The severity index is of primary interest but cutoff scores on the separate criterion measures could be of additional value to further document patients change patterns during treatment. Different scenarios relate to this issue. Criterion cutoff scores can possibly test the (manual-based) treatment assertion that specific BPD-problem areas must be addressed in a certain order or specific treatment phase. For example, if acting out in BPD is indeed addressed at start of a treatment, criterion 4 (self-destructive impulsivity) and 5 (parasuicidality) should show reductions in the first time frame. Scores for identity disturbance seen as a more fundamental feature of BPD are expected to diminish at much later times. For treatments aiming at specific symptomatic improvement instead of personality change, the application of one or some BPDSI-IV criteria could be sufficient and also more appropriate (e.g. Verheul, van den Bosch, et al., 2003). In this line, BPDSI-IV criterion cutoff scores were determined based on the BPDSI-IV study population, which can be found in Appendix II. 


\section{Clinical implications and future research}

The studies presented in this thesis demonstrate that BPD can be well and justifiably explained in a cognitive or a psychoanalytical theoretical framework and these frameworks can be fruitfully applied through SFT and TFP in the treatment of borderline patients.

The DSQ-48's validity and use in clinical practice are satisfactory to describe BPD-patients, as they are mostly characterized by an immature/primitive defensive style. When the overall structure of mature, neurotic and immature defensive styles is pursued for less defined patient groups, one should consider the possible limitations (chapter 3 and previous paragraph). The proposed 5-factor model (distrust, self-protection, self-sacrifice, avoidance and control) over 43 items may then be a better statistically coherent framework to describe patients' defense or coping style. The promising results on the BPDSI-IV and the BPDChecklist, support the use of these instruments in different mental health care settings and for different purposes as screening instrument, treatment outcome measure or for therapists' individual use.

In spite of all the positive findings and implications with respect to assessment instruments, one can also conclude that caution should be maintained since replication studies are needed, which is always the case with relatively new assessment instruments. In addition, further refinement and extension to other clinical control groups (especially Cluster B personality disorders, but also schizophrenics) is indicated. A limitation to all proposed cutoff scores and norms is that only one sample was used in calculating these scores. So, cutoff scores (including specificity and sensitivity) should also be re-examined in future research. Furthermore, future developments within the DSM borderline classification system will probably be adapted into new versions of the BPDSI and BPDChecklist. A different noteworthy remark involves the many promising responses on the initial report on the previous version of the BPDSI-IV (Arntz, van den Hoorn, et al., 2003). This not only exemplifies the need there is for BPD-specific assessment instruments, but also led to translations of the BPDSI-IV in Chinese, English, German, Hebrew, Italian, Korean and Norwegian. Consequently, new psychometric evaluations of the BPDSI-IV are currently in progress, with the valuable supplement that cross-cultural differences are simultaneously studied. The German and Italian BPDSI-IV already demonstrated excellent reliability and validity (personal communication). The BPDSI-IV is currently being used for research purposes in Australia, China, Germany, Israël, Italy, Norway, United Kingdom and the United States of America (personal communication).

Some cautionary notes should be made with respect to possible clinical implications after this borderline treatment study. The hiatus in available treatments for BPD-patients existed for a long time, but is now slowly being filled during the 
last decade with treatments for severely impaired BPD-patients as Dialectical Behavioral Therapy (Linehan, Armstrong, et al., 1991; Linehan, Heard, et al., 1993; Linehan, Schmidt, et al., 1999; Linehan, Tutek, Heard, et al., 1994) and Mentalization Based Treatment (Bateman \& Fonagy, 1999; 2001). As our study progressed, an increased eagerness and tendency to get ahead of the actual results was observed in the clinical field. Although the present study has positive results, and proved SFT to be more effective in treating BPD-patients than TFP, one cannot conclude that SFT is automatically the best evidence-based outpatient BPD-treatment and that TFP is only second choice. It must be emphasized that one RT never is a sufficient basis for guidelines. Replications of the present results are needed, as are (historical) comparisons of SFT and TFP to other manual-based treatments and so-called natural course/treatment-as-usual. Considering the Dutch situation in which the only psychotherapeutic treatments that the ministry of health, welfare and sport allows to be paid for by the generated health care funds are traditional psychodynamic treatments, it appears that comparing SFT to these traditional psychodynamic treatments is highly necessary. Furthermore, a historical comparison of the first year of SFT and TFP with a Dutch 1-year DBT efficacy study (Verheul, van den Bosch, et al., 2003) may be an interesting and realistic possibility as the initial plans for the grant application which funded the present RT, included a collaboration with the Dutch DBT study group. Methodological and practical problems eventually led to two separate studies but the main outcome measure (BPDSI-IV) remained the same.

The circumstances under which the present treatments were administered were, despite some methodological limitations, quite ideal. However, as already became clear, governmental and health care insurer's policies have changed during the study period, as a result of which the investigated SFT-treatment frequency and duration, is not realistic anymore for BPD-patients in current clinical practice. On the other hand, recent developments concerning the implementation of diagnostic-treatment-combinations (DBC's) throughout the Dutch health care system opens up new perspectives, as health insurers will have to negotiate with mental health care facilities. So, the generalizability of the results to the present mental health care situation and to other health care settings also has to be demonstrated in implementation studies. Another argument in this respect is that BPD-patients in outpatient settings are mostly female, like our study group, but that BPD-patients in other settings (e.g. forensic and addiction) are predominantly male. The number of male BPD-patients in the present study sample was too small to make any specific statements on differences in treatment results between men and women. A first development to investigate the generalizability of SFT with an implementation study of outpatient SFT for BPD in general psychiatry has recently started (Nadort, van Dyck, Spinhoven, et al., 2005). In the implementation study, SFT will consist of 50 sessions (within the present regula- 
tions) over 1.5 year. On another level, generalizability of SFT to other personality disorders than BPD will also be investigated in 2006 (Arntz, Severens, Evers, et al., 2005; Bernstein, 2006).

This SFT session-reduction introduces another issue; effectiveness of SFT and TFP is demonstrated for the manuals as applied in the treatment study but not for any other SFT/TFP-based treatments. When adjustments are made to the SFT or TFP manuals, for any reason, one should realize that the empirical support of adjusted SFT/TFP manuals cannot be fully based on the present SFT/TFP effectiveness study. Future research should determine how and which elements make SFT and TFP effective treatments.

Another point of attention for therapists who consider to additionally train in SFT or TFP, is that working with SFT or TFP adequately, requires much more training (and ongoing peer supervision and supervision) than many other manualbased treatments for Axis-I disorders. Apart from post-doctoral cognitivebehavioral or psychodynamical training, the reading of the manuals referred to will not automatically make a therapist a SFT- or TFP-therapist (Clarkin, Yeomans, et al., 1999; Young, Klosko, et al., 2003). A Dutch SFT-training has been developed, piloted and tested (Nadort, van Dyck, et al., 2005), and it could be concluded that a 50-hour training program, in combination with peer supervision and supervision, is a sufficient basis for training therapists in SFT. Furthermore, several Dutch regional post-doctoral training institutes (RINO's) started courses in which study therapists teach SFT and TFP. 



\section{References}




\section{References}

Adams HE, Bernat JA, \& Luscher KA (2001). Borderline personality disorder: an overview. In Sutker PB \& Adams HE (Eds.), Comprehensive Handbook of Psychopathology. New York: Kluwer Academic/Plenum Publishers.

Akaike H (1987). Factor analysis and AIC. Psychometrica, 52, 317-332.

Akiskal HS, Chen SE, Davis GC, Puzantian VR, Kashgarian M, \& Bolinger JM (1985). Borderline: an adjective in search of a noun. J Clin Psychiatry, 46(2), 41-8.

Andrews G, Pollock C, \& Stewart G (1989). The determination of defense style by questionnaire. Arch Gen Psychiatry, 46(5), 455-60.

Andrews G, Singh M, \& Bond M (1993). The Defense Style Questionnaire. J Nerv Ment Dis, $181(4), 246-56$.

Arntz A (1994a). Ambulante cognitieve gedragstherapie van de borderline persoonlijkheidsstoornis. In Derksen JJL \& Groen H (Eds.), Handboek voor de behandeling van borderline patiënten. Utrecht: De Tijdstroom.

Arntz A (1994b). Treatment of borderline personality disorder: a challenge for cognitivebehavioural therapy. Behav Res Ther, 32(4), 419-30.

Arntz A (1999). Do personality disorders exist? On the validity of the concept and its cognitivebehavioral formulation and treatment. Behav Res Ther, 37 Suppl 1, S97-134.

Arntz A (2004). Borderline personality disorder. In Beck AT, Freeman A, Davis DD, Pretzer J, Fleming B, Arntz A, \& al. e (Eds.), Cognitive therapy of personality disorders (pp. 187215). New York Guilford press.

Arntz A (2005). Introduction to special issue: cognition and emotion in borderline personality disorder. J Behav Ther Exp Psychiatry, 36(3), 167-72.

Arntz A, Dietzel R, \& Dreessen L (1999). Assumptions in borderline personality disorder: specificity, stability and relationship with etiological factors. Behav Res Ther, 37(6), 545-57.

Arntz A, Dreessen L, \& Giesen-Bloo J (1999). Borderline Personality Disorder Checklist. Maastricht Author.

Arntz A, Dreessen L, Schouten E, \& Weertman A (2004). Beliefs in personality disorders: a test with the personality disorder belief questionnaire. Behav Res Ther, 42(10), 1215-25.

Arntz A, \& Kerkhofs MLM (1999). Inventarisatie van de persoonlijkheidsstructuur (Authorized Dutch translation of the Inventory of Personality Organization). Maastricht: Author.

Arntz A, \& Kuipers $H$ (1998). Cognitieve gedragstherapie bij de borderline persoonlijkheidsstoornis. In van Tilburg W, van den Brink W, \& Arntz A (Eds.), Behandelingsstrategieën bij de borderline persoonlijkheidsstoornis. Houten: Bohn Stafleu Van Loghum.

Arntz A, Severens JL, Evers S, Spinhoven P, Bernstein D, Widdershoven G, \& Abma T (2005). Psychological treatment of personality disorders: a multicentered randomized controlled trial on the (cost-)effectiveness of schema-focused therapy. Maastricht: Author.

Arntz A, van den Hoorn M, Cornelis J, Verheul R, van den Bosch WM, \& de Bie AJ (2003). Reliability and validity of the borderline personality disorder severity index. J Personal Disord, 17(1), 45-59.

Arrindell WA, \& Ettema JHM (1986). Klachtenlijst (SCL-90). Lisse: Swets \& Zeitlinger.

APA (1980). Diagnostic and statistical manual of mental disorders (3rd ed.). Washington DC: Author.

APA (1994). Diagnostic and statistical manual of mental disorders (4th ed.). Washington DC: Author. 
APA (2000). Diagnostic and statistical manual of mental disorders (4th ed., text revision). Washington DC: Author.

APA (2001). Practice guideline for the treatment of patients with borderline personality disorder. American Psychiatric Association. Am J Psychiatry, 158(10 Suppl), 1-52.

Ayers WA (2000). Taxometric analysis of borderline and antisocial personality disorders in a drug and alcohol dependent population. Dissertation abstracts international, 61, 1684.

Barber JP, Connolly MB, Crits-Christoph P, Gladis L, \& Siqueland L (2000). Alliance predicts patients' outcome beyond in-treatment change in symptoms. J Consult Clin Psychol, 68(6), 1027-32.

Baron RM, \& Kenny DA (1986). The moderator-mediator variable distinction in social psychological research: conceptual, strategic, and statistical considerations. J Pers Soc Psychol, $51(6), 1173-82$.

Bateman A, \& Fonagy P (1999). Effectiveness of partial hospitalization in the treatment of borderline personality disorder: a randomized controlled trial. Am J Psychiatry, 156(10), 15639.

Bateman A, \& Fonagy P (2001). Treatment of borderline personality disorder with psychoanalytically oriented partial hospitalization: an 18-month follow-up. Am J Psychiatry, 158(1), 36 42.

Bateman A, \& Fonagy P (2004). Psychotherapy for borderline personality disorder: mentalizationbased treatment. Oxford, UK: Oxford University Press.

Beck AT, Freeman A, \& Associates (1990). Cognitive therapy of personality disorders. New York: Guilford Press.

Beck AT, Freeman A, Davis DD, Pretzer J, Fleming B, Arntz A, \& al. e (2004). Cognitive therapy of personality disorders (2nd ed.). New York: The Guilford Press.

Benjamin LS (1996). Interpersonal diagnosis and treatment of personality disorders. (2nd ed.). New York: Guilford Press.

Bernstein DP (2006). Adaptation, implementation, and initial (one year) efficacy of schema therapy for TBS patients with personality disorders. Maastricht: Author.

Berry KL, Miskimins RW, \& Wilson LT (1972). The concept of self and psychiatric chronicity. J Clin Psychol, 28(1), 5-8.

Berry WG, \& Sipps GJ (1991). Interactive effects of counselor-client similarity and client selfesteem on termination type and number of sessions. Journal of Counseling Psychology, 38, 120-125.

Beutler LE, Malik M, Alimohamed S, Harwood TM, Talebi H, Noble S, \& al. e (2004). Therapist variables. In Lambert MJ (Ed.), Bergin and Garfield's Handbook of psychotherapy and behavioral change (fifth ed.) (pp. 227-306). New York: Wiley

Blascovich J, \& Tomaka J (1991). Measures of self-esteem. In Robinson JP, Shaver PR, \& Wrightsman LS (Eds.), Measures in personality and social psychological attitudes (pp. 115160). New York: Academic Press.

Blum N, Pfohl B, John DS, Monahan P, \& Black DW (2002). STEPPS: a cognitive-behavioral systems-based group treatment for outpatients with borderline personality disorder--a preliminary report. Compr Psychiatry, 43(4), 301-10.

Bond M, Gardner ST, Christian J, \& Sigal JJ (1983). Empirical study of self-rated defense styles. Arch Gen Psychiatry, 40(3), 333-8.

Bond M, Paris J, \& Zweig-Frank H (1994). Defense styles and borderline personality disorder. J Personal Disord, 8, 28-31. 
Boon SB, \& Draijer N (1995). Screening en diagnostiek van dissociatieve stoornissen. Lisse: Swets \& Zeitlinger.

Bowlby J (1980). Attachment and loss (Vol. 2). New York: Basic Books.

Briggs SR, \& Cheek JM (1986). The role of factor analysis in the development and evaluation of personality scales. J Pers, 54, 106-148.

Brophy CJ, Norvell NK, \& Kiluk DJ (1988). An examination of the factor structure and convergent and discriminant validity of the SCL-90R in an outpatient clinic population. J Pers Assess, 52(2), 334-40.

Brown GK, Newman CF, Charlesworth SE, Crits-Christoph P, \& Beck AT (2004). An open clinical trial of cognitive therapy for borderline personality disorder. J Personal Disord, 18(3), 257 71.

Brunink SA, \& Schroeder HE (1979). Verbal therapeutic behavior of expert psychoanalytically oriented, Gestalt, and behavior therapists. J Consult Clin Psychol, 47(3), 567-74.

Burns JW, Kubilus A, Bruehl S, Harden RN, \& Lofland K (2003). Do changes in cognitive factors influence outcome following multidisciplinary treatment for chronic pain? A cross-lagged panel analysis. J Consult Clin Psychol, 71(1), 81-91.

Cattell RB (1966). The scree test for the number of factors. Multivariate Behavioral Research, 1, 245-276.

Chiesa M, Fonagy P, Holmes J, Drahorad C, \& Harrison-Hall A (2002). Health service use costs by personality disorder following specialist and nonspecialist treatment: a comparative study. J Personal Disord, 16(2), 160-73.

Clarkin JF, Foelsch PA, Levy KN, Hull JW, Delaney JC, \& Kernberg OF (2001). The development of a psychodynamic treatment for patients with borderline personality disorder: a preliminary study of behavioral change. J Personal Disord, 15(6), 487-95.

Clarkin JF, Hull JW, Cantor J, \& Sanderson C (1993). Borderline personality disorder and personality traits: a comparison of SCID-II BPD and NEO-PI. Psychol Assess, 5, 472-476.

Clarkin JF, Hull JW, \& Hurt SW (1993). Factor structure of borderline personality disorder criteria. J Personal Disord, 7, 137-143.

Clarkin JF, Koenigsberg H, Yeomans F, Selzer M, Kernberg P, \& Kernberg OF (1994). Psychodynamische psychotherapie bij de borderline patiënt. In Derksen JJL \& Groen H (Eds.), Handboek voor de behandeling van borderline patiënten. Utrecht: De Tijdstroom.

Clarkin JF, Levy KN, Lenzenweger MF, \& Kernberg OF (2004). The Personality Disorders Institute/Borderline Personality Disorder Research Foundation randomized control trial for borderline personality disorder: rationale, methods, and patient characteristics. J Personal Disord, 18(1), 52-72.

Clarkin JF, Yeomans FE, \& Kernberg OF (1999). Psychotherapy for borderline personality. New York: John Wiley \& Sons.

Cloninger CR (2000). A practical way to diagnosis personality disorder: a proposal. J Personal Disord, 14(2), 99-108.

Coccaro EF, \& Siever LJ (2005). Neurobiology. In Oldham JM, Skodol AE, \& Bender DS (Eds.), Textbook of personality disorders. Washington DC: The American Psychiatric Publishing Inc.

Cooper SH (1998). Changing notions of defense within psychoanalytic theory. J Pers, 66, 947964.

Costa Jr. PT, \& McCrae RR (1990). Personality disorders and the five-factor model of personality J Personal Disord, 4, 362-371. 
Costa Jr. PT, \& McCrae RR (1992). The five-factor model of personality and its relevance to personality disorders. J Personal Disord, 6, 343-359.

Cramer P (1999). Personality, personality disorders, and defense mechanisms. J Pers, 67, 535554.

Cramer P, \& Blatt SJ (1990). Use of the TAT to measure change in defense mechanisms following intensive psychotherapy. J Pers Assess, 54(1-2), 236-51.

Crits-Christoph P, \& Mintz J (1991). Implications of therapist effects for the design and analysis of comparative studies of psychotherapies. J Consult Clin Psychol, 59(1), 20-6.

Cronbach LJ, \& Gleser GC (1953). Assessing similarity between profiles. Psychol Bull, 50(6), 456-73.

Davidson K, \& MacGregor MW (1996). Reliability of an idiographic Q-sort measure of defense mechanisms. J Pers Assess, 66(3), 624-39.

de Jong CAJ (1998). IBO-interpersonal behavior observation. Sint Oedenrode NL: NovadicKentron, Network for addiction treatment services.

de Jong CAJ, Derks F, van Oel C, \& Rinne T (1996). Gestructureerd Interview voor de DSM-IV Persoonlijkheidsstoornissen. Sint Oedenrode: Stichting Verslavingszorg Oost Brabant.

de Jong CAJ, van den Brink W, \& Jansma A (2000). ICL-R: Handleiding bij de vernieuwde Nederlandse versie van de Interpersonal Checklist. Sint Oedenrode NL: Novadic-Kentron, Network for addiction treatment services.

Derksen JJL (1988). Het diagnostisch interview voor borderline patiënten: handleiding (DIB). Lisse: Swets Test Services.

Derksen JJL, Bender J, \& Roverts J (1994). De psychologische behandeling van de borderline patiënt. In Derksen JJL \& Groen H (Eds.), Handboek voor de behandeling van borderline patiënten. Utrecht: De Tijdstroom.

Derksen JJL, \& Groen H (Eds.). (1994). Handboek voor de behandeling van borderline patiënten. Utrecht: De Tijdstroom.

Derksen JJL, Hummelen JW, \& Bouwens JMP (1988). Structurele diagnostiek en het structureel interview. Tijdschrift voor Psychiatrie, 30, 445-459.

Derogatis LR, Lipman RS, \& Covi L (1973). SCL-90: an outpatient psychiatric rating scale-preliminary report. Psychopharmacol Bull, 9(1), 13-28.

Devens M, \& Erickson MT (1998). The relationship between defense styles and personality disorders. J Personal Disord, 12(1), 86-93.

Draijer N, \& Boon S (1993). The validation of the dissociative experiences scale against the criterion for the SCID-I, using receiver operating characteristics $(\mathrm{ROC})$ analysis. Dissociation, 6 , 28-37.

Dreessen L, Stroux A, \& Weckx M (1998). Nederlandse vertaling van het gestructureerd klinisch interview voor DSM-IV kind versie (KID-SCID; versie 1.0). Maastricht: Author.

EuroQol Group TE (1990). EuroQol--a new facility for the measurement of health-related quality of life. The EuroQol Group. Health Policy, 16(3), 199-208.

Evon DM, \& Burns JW (2004). Process and outcome in cardiac rehabilitation: an examination of cross-lagged effects. J Consult Clin Psychol, 72(4), 605-16.

Finkel SE (1995). Causal analysis with panel data. Thousands Oaks CA: Sage.

First MB, Gibbon M, Spitzer RL, Williams JBW, \& Benjamin LS (1997). User's guide for the structured clinical interview for DSM-IV axis II personality disorders (SCID-II). Washington DC: American Psychiatric Press. 
First MB, Spitzer RL, Gibbon M, \& Williams JBW (1996). Structured clinical interview for DSM-IV axis I disorders - patient edition (SCID-I/P), version 2.0. New York: Biometrics Research Department, New York State Psychiatric Institute.

Fonagy $P$ (2000). Attachment and borderline personality disorder. Journal of the American Psychoanalytic Association, 48, 1129-1146.

Fossati A, Madeddu F, \& Maffei C (1999). Borderline Personality Disorder and childhood sexual abuse: a meta-analytic study. J Personal Disord, 13(3), 268-80.

Fossati A, Maffei C, Bagnato M, Donati D, Namia C, \& Novella L (1999). Latent structure analysis of DSM-IV borderline personality disorder criteria. Compr Psychiatry, 40(1), 72-9.

Frances A (1982). Categorical and dimensional systems of personality diagnosis: a comparison. Compr Psychiatry, 23(6), 516-27.

Gaston L, Marmar CR, Gallagher D, \& Thompson LW (1991). Alliance prediction of outcome beyond in-treatment symptomatic change as psychotherapy progresses. Psychotherapy Research, 1, 104-112.

Giesen-Bloo J (2001). Wereld Assumpties Schaal (Dutch version of the World Assumptions Scale). Maastricht: Author.

Giesen-Bloo J, \& Arntz A (2005). World assumptions and the role of trauma in borderline personality disorder. J Behav Ther Exp Psychiatry, 36(3), 197-208.

Giesen-Bloo J, Arntz A, Opdenacker J, \& Spinhoven P (2006). The defense style questionnaire 48: factor structure in clinical and nonclinical samples. Submitted for publication.

Giesen-Bloo J, Arntz A, van Dyck R, Spinhoven P, \& van Tilburg W (2002, November 2002). Outpatient treatment of borderline personality disorder: analytical psychotherapy versus cognitive behavior therapy. Paper presented at the Transference Focused Psychotherapy for Borderline Personality Symposium, November 16-17, New York, USA.

Giesen-Bloo J, van Dyck R, Spinhoven P, van Tilburg W, Dirksen C, van Asselt A, Kremers I, Nadort $M$, \& Arntz $A$ (2006). Outpatient psychotherapy for borderline personality disorder: a randomized trial of schema focused therapy versus transference focused psychotherapy. Arch Gen Psychiatry, 63, 649-58.

Giesen-Bloo JH, Arntz A, \& Schouten E (2006). The borderline personality disorder checklist: psychometric evaluation and factorial structure in clinical and nonclinical samples. Submitted for publication.

Giesen-Bloo JH, Arntz A, van Dyck R, Spinhoven P, \& van Tilburg W (2001). Outpatient treatment of borderline personality disorder: analytical psychotherapy versus cognitive behavior therapy. Paper presented at the World Congress of Behavioral and Cognitive Therapies, July 17-21, Vancouver, Canada.

Giesen-Bloo JH, Wachters LM, Schouten E, \& Arntz A (2006). Assessment of borderline personality disorder with the borderline personality disorder severity index-IV: psychometric evaluation and dimensional structure. Submitted for publication.

Gitlin MJ (1993). Pharmacotherapy of personality disorders: conceptual framework and clinical strategies. J Clin Psychopharmacol, 13(5), 343-53.

Gluhoski VL, \& Wortman CB (1996). The impact of trauma on world views. J Soc Clin Psychology, $15,417-429$.

Groenestijn MAC, Akkerhuis GW, Kupka RW, Schneider N, \& Nolen WA (1999). Gestructureerd klinisch interview voor de vaststelling van DSM-IV as I stoornissen. Lisse: Swets \& Zeitlinger. 
Guadagnoli E, \& Velicer WF (1988). Relation of sample size to the stability of component patterns. Psychol Bull, 103(2), 265-75.

Gunderson JG (2000). Psychodynamic psychotherapy for borderline personality disorder. In Gunderson JG \& Gabbard GO (Eds.), Psychotherapy for personality disorders (pp. 33-64). Washington DC: American Psychiatric Press.

Gunderson JG, \& Phillips KA (1991). A current view of the interface between borderline personality disorder and depression. Am J Psychiatry, 148(8), 967-75.

Gunderson JG, \& Sabo AN (1993). The phenomenological and conceptual interface between borderline personality disorder and PTSD. Am J Psychiatry, 150(1), 19-27.

Gunderson JG, \& Singer MT (1975). Defining borderline patients: an overview. Am J Psychiatry, $132(1), 1-10$.

Gunderson JG, \& Zanarini MC (1987). Current overview of the borderline diagnosis. J Clin Psychiatry, 48 Suppl, 5-14.

Hahn SR, Thompson KS, Stern V, Budner NS, \& Wills TA (1990). The difficult doctor-patientrelationship questionnaire - assessing the problem patient. Clinical Research, 38, A739.

Hare RD, Hart SD, \& Harpur TJ (1991). Psychopathy and the DSM-IV criteria for antisocial personality disorder. J Abnorm Psychol, 100(3), 391-8.

Haslam N (2002). The dimensional view of personality disorders: a review of the taxometric evidence. Clin Psychol Review, 23, 75-93.

Henry WP, Schacht TE, \& Strupp HH (1990). Patient and therapist introject, interpersonal process, and differential psychotherapy outcome. J Consult Clin Psychol, 58(6), 768-74.

Herman JL, Perry JC, \& van der Kolk BA (1989). Childhood trauma in borderline personality disorder. Am J Psychiatry, 146(4), 490-5.

Herman SM (1998). The relationship between therapist-client modality similarity and psychotherapy outcome. Journal of Psychotherapy Practice and Research, 7, 56-64.

Hilliard RB, Henry WP, \& Strupp HH (2000). An interpersonal model of psychotherapy: linking patient and therapist developmental history, therapeutic process, and types of outcome. $J$ Consult Clin Psychol, 68(1), 125-33.

Hoglend $P$ (1993). Personality disorders and long-term outcome after brief dynamic psychotherapy. J Personal Disord, 7, 168-181.

Horvath AO, \& Greenberg LS (1989). Development and validation of the working alliance inventory. Journal of Counseling Psychology, 36, 223-233.

Horvath AO, \& Symonds BD (1991). Relationship between working alliance and outcome in psychotherapy: a meta-analyses. Journal of Counseling Psychology, 38, 139-149.

Hurt S, Clarkin J, Widiger T, Fyer M, Sullivan T, Stone M, \& Frances A (1990). Evaluation of DSM III decision rules for case detection using joint conditional probability structures. J Personal Disord, 4, 121-130.

Ingenhoven T, \& van den Brink W (1994). Borderline persoonlijkheidsstoornis: diagnostische en epidemiologische aspecten. In Derksen JJL \& Groen H (Eds.), Handboek voor de behandeling van borderline patiënten. Utrecht: De Tijdstroom.

Jacobson NS, \& Truax P (1991). Clinical significance: a statistical approach to defining meaningful change in psychotherapy research. J Consult Clin Psychol, 59(1), 12-9.

Janoff-Bulman R (1989). Assumptive worlds and the stress of traumatic events: applications of the schema construct. Social Cognition, 7, 113-136. 
Janoff-Bulman R (1991). Understanding people in terms of their assumptive worlds. In Ozer D, Healy J, \& Stewart A (Eds.), Perspectives in personality: self and emotion (pp. 99-116). London: Kingsley.

Janoff-Bulman R (1992). Shattered assumptions: towards a new psychology of trauma. New York: The Free Press.

Kenny DA, \& La Voie L (1985). Separating individual and group effects. J Pers Soc Psychol, 38(249-256).

Kernberg OF (1984). Severe personality disorders. New Haven CT: Yale University Press.

Kernberg OF (1996). A psychoanalytic theory of personality disorders. In Clarkin JF \& Lenzenweger MF (Eds.), Major theories of personality disorders (pp. 106-140). New York Guilford Press.

Kernberg OF, \& Clarkin JF (1995). The Inventory of Personality Organization. White Plains NY: The New York Hospital - Cornell Medical Center.

Kernberg OF, Selzer MA, Koenigsberg HW, Carr AC, \& Appelbaum AH (1989). Psychodynamic psychotherapy of borderline patients. New York: Basic Books.

Kiers HAL (1990). SCA: a program for simultaneous components analysis of variables measured in two or more populations. Groningen (The Netherlands): iec ProGAMMA.

Kiesler DJ (1996). Contemporary interpersonal theory and research: personality, psychopathology, and psychotherapy. Oxford GB: Wiley.

Kiesler DJ, \& Watkins LM (1989). Interpersonal complementarity and the therapeutic alliance: a study of relationship in psychotherapy. Psychotherapy:Theory, Research and Practice, 26, 183-194.

Koenigsberg HW, Kernberg OF, Stone MH, Appelbaum AH, Yeomans FE, \& Diamond DD (2000). Borderline patients: extending the limits of treatability. New York: Basic Books.

Kooij JJS (1997). Screeningslijst voor aandachtstekortstoornis met hyperactiviteit bij volwassenen. Delft: Institute for Mental Health Delfland.

Kremers IP (2004). Remembering autobiographical events in borderline personality disorder: Dissertation Leiden University.

Kullgren G, \& Armelius B (1990). The concept of borderline personality organization: a long-term comparative follow-up study with special reference to borderline personality organization. J Personal Disord, 4, 203-212.

Laforge R, \& Suczek RF (1955). The interpersonal dimension of personality. III. An interpersonal check list. J Pers, 24(1), 94-112.

Lambert MJ (1989). The individual therapist's contribution to psychotherapy process and outcome. Clin Psychol Review, 9, 469-485.

Lambert MJ, Hansen NB, \& Finch AE (2001). Patient-focused research: using patient outcome data to enhance treatment effects. J Consult Clin Psychol, 69(2), 159-72.

Layden MA, Newman CF, Freeman A, \& Byers Morse S (1993). Cognitive therapy of borderline personality disorder. Boston: Allyn \& Bacon.

Lenzenweger MF, \& Clarkin JF (1996). The personality disorder: history, classification and research issues. In Clarkin JF \& Lenzenweger MF (Eds.), Major theories of personality disorders (pp. 1-35). New York: Guilford.

Lenzenweger MF, Clarkin JF, Kernberg OF, \& Foelsch PA (2001). The Inventory of Personality Organization: psychometric properties, factorial composition, and criterion relations with affect, aggressive dyscontrol, psychosis proneness, and self-domains in a nonclinical sample. Psychol Assess, 13(4), 577-91. 
Lerner H, Albert C, \& Walsh M (1987). The rorschach assessment of borderline defenses: a concurrent validity study. J Pers Assess, 51(3), 334-54.

Lieb K, Zanarini MC, Schmahl C, Linehan MM, \& Bohus M (2004). Borderline personality disorder. Lancet, 364(9432), 453-61.

Linehan MM (1993a). Cognitive-behavioral treatment of borderline personality disorder. New York: Guilford Press.

Linehan MM (1993b). The skills training manual for treating borderline personality disorder. New York: The Guilford Press.

Linehan MM, Armstrong HE, Suarez A, Allmon D, \& Heard HL (1991). Cognitive-behavioral treatment of chronically parasuicidal borderline patients. Arch Gen Psychiatry, 48(12), 1060-4.

Linehan MM, \& Heard H (1999). Borderline personality disorder: costs, course, and treatment outcomes. In Miller NE \& Magruder KM (Eds.), Cost-effectiveness of psychotherapy: a guide for practitioners, researchers, and policymakers (pp. 291-305). New York: Oxford University Press.

Linehan MM, Heard HL, \& Armstrong HE (1993). Naturalistic follow-up of a behavioral treatment for chronically parasuicidal borderline patients. Arch Gen Psychiatry, 50(12), 971-4.

Linehan MM, Schmidt H, 3rd, Dimeff LA, Craft JC, Kanter J, \& Comtois KA (1999). Dialectical behavior therapy for patients with borderline personality disorder and drug-dependence. Am J Addict, 8(4), 279-92.

Linehan MM, Tutek DA, Heard HL, \& Armstrong HE (1994). Interpersonal outcome of cognitive behavioral treatment for chronically suicidal borderline patients. Am J Psychiatry, 151(12), 1771-6.

Lingiardi V, Lonati C, Delucchi F, Fossati A, Vanzulli L, \& Maffei C (1999). Defense mechanisms and personality disorders. J Nerv Ment Dis, 187(4), 224-8.

Links PS, Heslegrave R, \& van Reekum R (1998). Prospective follow-up study of borderline personality disorder: prognosis, prediction of outcome, and Axis II comorbidity. Can J Psychiatry, 43(3), 265-70.

Livesley WJ (2001). Conceptual and taxonomic issues. In Livesley WJ (Ed.), Handbook of personality disorders (pp. 3-38). New York Guilford.

Livesley WJ, Jang KL, \& Vernon PA (1998). Phenotypic and genetic structure of traits delineating personality disorder. Arch Gen Psychiatry, 55(10), 941-8.

Livesley WJ, \& Schroeder ML (1991). Dimensions of personality disorder. The DSM-III-R cluster B diagnoses. J Nerv Ment Dis, 179(6), 317-28.

Luborsky L (1984). Principals of psychoanalytic psychotherapy: a manual for supportiveexpressive treatment. New York: Basic Books.

Lutz W, Rafaeli E, Howard KI, \& Martinovich Z (2002). Adaptive Modeling of Progress in Outpatient Psychotherapy. Psychotherapy Research, 12, 427-443.

Lynam DR, \& Widiger TA (2001). Using the five-factor model to represent the DSM-IV personality disorders: an expert consensus approach. J Abnorm Psychol, 110(3), 401-12.

MacCallum RC, \& Widaman KF (1999). Sample size in factor analysis. Psychol Methods, 4, 8499.

Marmar CR, Gaston L, Gallagher D, \& Thompson LW (1989). Alliance and outcome in late-life depression. J Nerv Ment Dis, 177(8), 464-72.

Martin DJ, Garske JP, \& Davis MK (2000). Relation of the therapeutic alliance with outcome and other variables: a meta-analytic review. J Consult Clin Psychol, 68(3), 438-50. 
Marziali E, \& Munroe-Blum H (1994). Interpersonal group psychotherapy for borderline personality disorder. New York: Basic Books.

McDermut W, \& Zimmerman M (2005). Assessment instruments and standardized evaluation. In Oldham JM, Skodol AE, \& Bender DS (Eds.), Textbook of personality disorders. Washington DC: The American Psychiatric Publishing Inc.

McGlashan TH (1986). The Chestnut Lodge follow-up study. III. Long-term outcome of borderline personalities. Arch Gen Psychiatry, 43(1), 20-30.

McGlashan TH (2002). The borderline personality disorder practice guidelines: the good, the bad, and the realistic. J Personal Disord, 16(2), 119-21.

Meares R (2004). The conversational model: an outline. Am J Psychother, 58(1), 51-66.

Millon T, \& Grossman SD (2005). Sociocultural factors. In Oldham JM, Skodol AE, \& Bender DS (Eds.), Textbook of personality disorders. Washington DC: The American Psychiatric Publishing Inc.

Millsap RE, \& Meredith W (1988). Component analysis in cross-sectional and longitudinal data. Psychometrica, 53, 123-134.

Miskimins RW, \& Baker BR (1973). Self concept and the disadvantages. J Commun Psychol, 1, 347-361.

Miskimins RW, Wilson LT, Braucht GN, \& Berry KL (1971). Self-concept and psychiatric symptomatology. J Clin Psychol, 27(2), 185-7.

Morey LC (1991). Personality Assessment Inventory: professional manual. Odessa FL: Psychological Assessment Resources.

Morey LC, Alexander GM, \& Boggs C (2005). Gender. In Oldham JM, Skodol AE, \& Bender DS (Eds.), Textbook of personality disorders. Washington DC: The American Psychiatric Publishing Inc.

Morrison N (2000). Schema-focused cognitive therapy for complex long-standing problems: a single case study. Behavioural and Cognitive Psychotherapy, 28, 269-283.

Munroe-Blum H, \& Marziali E (1995). A controlled trial of short-term group treatment for borderline personality disorder. J Personal Disord, 9, 190-198.

Muris P, \& Merckelbach H (1994). Defense style, trait anxiety, worry, and bodily symptoms. Personal Ind Diff, 16, 349-351.

Nadort M, van Dyck R, Spinhoven P, Wensing M, Smit J, Arntz A, \& al. e (2005). Implementation of outpatient schema focused therapy for borderline personality disorder in general psychiatry. Amsterdam: ZonMw.

Nahas Z, Molnar C, \& George MS (2005). Brain Imaging. In Oldham JM, Skodol AE, \& Bender DS (Eds.), Textbook of personality disorders. Washington DC: The American Psychiatric Publishing Inc.

Nelson-Gray RO, \& Farmer RF (1999). Behavioral assessment of personality disorders. Behav Res Ther, 37(4), 347-68.

Nordahl HM, \& Nysaeter TE (2005). Schema therapy for patients with borderline personality disorder: a single case series. J Behav Ther Exp Psychiatry, 36(3), 254-64.

Nunnally JC (1978). Psychometric theory. (2nd ed.). New York: Mc Graw-Hill.

Oldham JM, Gabbard GO, Goin MK, Gunderson JG, Soloff P, Spiegel D, \& al. e (2001). Practice guideline for treatment of patients with borderline personality disorder. Am J Psychiatry, $158,1-52$

Oldham JM, Skodol AE, Kellman HD, Hyler SE, Doidge N, Rosnick L, \& Gallaher PE (1995). Comorbidity of axis I and axis II disorders. Am J Psychiatry, 152(4), 571-8. 
Orlinsky DE, Ronnestad MH, \& Willutzki U (2004). Fifty years of psychotherapy process-outcome research: continuity and change. In Lambert MJ (Ed.), Bergin and Garfield's Handbook of psychotherapy and behavioral change (fifth ed.) (pp. 307-390). New York: Wiley.

Paddock JR, \& Nowicki S, Jr. (1986). The circumplexity of Leary's Interpersonal Circle: a multidimensional scaling perspective. J Pers Assess, 50(2), 279-89.

Paris $\mathrm{J}$ (1993). The treatment of borderline personality disorder in light of the research on its long term outcome. Can J Psychiatry, 38 Suppl 1, S28-34.

Paris $\mathrm{J}$ (1996). Social factors in the personality disorders. A biopsychosocial approach to etiology and treatment. Cambridge UK: Cambridge University Press.

Paris J (2002). Commentary on the American Psychiatric Association guidelines for the treatment of borderline personality disorder: evidence-based psychiatry and the quality of evidence. J Personal Disord, 16(2), 130-4.

Paris $\mathrm{J}$ (2005). A current integrative perspective on personality disorders. In Oldham JM, Skodol $A E$, \& Bender DS (Eds.), Textbook of personality disorders. Washington DC: The American Psychiatric Publishing Inc.

Paris J, Brown R, \& Nowlis D (1987). Long-term follow-up of borderline patients in a general hospital. Compr Psychiatry, 28(6), 530-5.

Paris J, \& Zweig-Frank H (2001). A 27-year follow-up of patients with borderline personality disorder. Compr Psychiatry, 42(6), 482-7.

Paris J, Zweig-Frank H, Bond M, \& Guzder J (1996). Defense styles, hostility, and psychological risk factors in male patients with personality disorders. J Nerv Ment Dis, 184(3), 153-8.

Parkes CM (1971). Psycho-social transitions: a field for study. Soc Sci Med, 5(2), 101-15.

Perry JC, Banon E, \& lanni F (1999). Effectiveness of psychotherapy for personality disorders. Am J Psychiatry, 156(9), 1312-21.

Perry JC, \& Cooper SH (1989). An empirical study of defense mechanisms. I. Clinical interview and life vignette ratings. Arch Gen Psychiatry, 46(5), 444-52.

Perry JC, \& Hoglend P (1998). Convergent and discriminant validity of overall defensive functioning. J Nerv Ment Dis, 186(9), 529-35.

Perry JC, \& lanni FF (1998). Observer-rated measures of defense mechanisms. J Pers, 66, 9931024.

Pfohl B, Blum N, \& Zimmerman M (1997). Structured Interview for DSM-IV Personality. Washington DC: American Psychiatric Press.

Pollock C, \& Andrews G (1989). Defense styles associated with specific anxiety disorders. Am J Psychiatry, 146(11), 1500-2.

Pretzer J (1990). Borderline personality disorder. In Beck AT, Freeman A, \& Associates (Eds.), Cognitive therapy of personality disorders. New York: Guilford Press.

Raue PJ, Goldfried MR, \& Barkham M (1997). The therapeutic alliance in psychodynamicinterpersonal and cognitive-behavioral therapy. J Consult Clin Psychol, 65(4), 582-7.

Regan AM, \& Hill CE (1992). Investigations of what clients and counselors do not say in brief therapy. Journal of Counseling Psychology, 39, 168-174.

Rijkeboer MM, van den Bergh H, \& van den Bout J (2005). Stability and discriminative power of the Young Schema-Questionnaire in a Dutch clinical versus non-clinical population. J Behav Ther Exp Psychiatry, 36(2), 129-44.

Rockland LH (1992). Supportive therapy for borderline patients. New York: The Guilford Press.

Rosenberg M (1965). Society and the adolescent self-image. Princeton: University Press.

Rosenberg M (1979). Conceiving the self. New York: Basic Books. 
Rothschild L, Cleland C, Haslam N, \& Zimmerman M (2003). A taxometric study of borderline personality disorder. J Abnorm Psychol, 112(4), 657-66.

Ryle A (1997). Cognitive analytic therapy and borderline personality disorder: the model and the method. Chichester UK: Wiley \& Sons.

Ryle A, \& Golynkina K (2000). Effectiveness of time-limited cognitive analytic therapy of borderline personality disorder: factors associated with outcome. $\mathrm{Br} J$ Med Psychol, 73 (Pt 2), 197-210.

Sabo AN (1997). Etiological significance of associations between childhood trauma and borderline personality disorder: conceptual and clinical implications. J Personal Disord, 11(1), 5070.

Safran JD, Muran JC, Samstag LW, \& Stevens C (2001). Repairing alliance ruptures. Psychotherapy: Theory, Research, Practice, Training, 38, 406-412.

Salvio MA, Beutler LE, Wood JM, \& Engle D (1992). The strength of the therapeutic alliance in three treatments of depression. Psychotherapy Research, 2, 31-36.

Sammallahti P, \& Aalberg $V$ (1995). Defense style in personality disorders. An empirical study. J Nerv Ment Dis, 183(8), 516-21.

Sammallahti P, Aalberg V, \& Pentinsaari JP (1994). Does defense style vary with severity of mental disorder? An empirical assessment. Acta Psychiatr Scand, 90(4), 290-4.

Samstag LW, Batchelder ST, Muran JC, Safran JD, \& Winston A (1998). Early identification of treatment failures in short-term psychotherapy. An assessment of therapeutic alliance and interpersonal behavior. J Psychother Pract Res, 7(2), 126-43.

Sandell R, Alfredsson E, Berg M, Crafoord K, Lagerlof A, Arkel I, Cohn T, Rasch B, \& Rugolska A (1993). Clinical significance of outcome in long-term follow-up of borderline patients a $a$ day hospital. Acta Psychiatr Scand, 87(6), 405-13.

Sanderson C, Swenson C, \& Bohus M (2002). A critique of the American psychiatric practice guideline for the treatment of patients with borderline personality disorder. J Personal Disord, 16(2), 122-9.

Sanislow CA, Grilo CM, \& McGlashan TH (2000). Factor analysis of the DSM-III-R borderline personality disorder criteria in psychiatric inpatients. Am J Psychiatry, 157(10), 1629-33.

Sanislow CA, Grilo CM, Morey LC, Bender DS, Skodol AE, Gunderson JG, Shea MT, Stout RL, Zanarini MC, \& McGlashan TH (2002). Confirmatory factor analysis of DSM-IV criteria for borderline personality disorder: findings from the collaborative longitudinal personality disorders study. Am J Psychiatry, 159(2), 284-90.

Saulsman LM, \& Page AC (2004). The five-factor model and personality disorder empirical literature: A meta-analytic review. Clin Psychol Rev, 23(8), 1055-85.

Schmand B, Lindeboom J, \& van Harskamp F (1992). NLV, Nederlandse Leestest voor Volwassenen. Lisse: Swets \& Zeitlinger.

Schmidt NB, Joiner TE, Young JE, \& Telch MJ (1995). The schema questionnaire: investigation of psychometric properties and the hierarchical structure of a measure of maladaptive schemas. Cogn Ther Res, 19, 231-295.

Schouten HJ (1995). Adaptive biased urn randomization in small strata when blinding is impossible. Biometrics, 51(4), 1529-35.

Shearin EN, \& Linehan MM (1992). Patient-therapist ratings and relationship to progress in dialectical therapy for borderline personality disorder. Behav Ther, 23, 730-741.

Shedler J, \& Westen D (2004). Refining personality disorder diagnosis: integrating science and practice. Am J Psychiatry, 161(8), 1350-65. 
Sieswerda SH (2006). On hypervigilance and extreme evaluations in borderline personality disorder. University Maastricht: Dissertation in preparation.

Simpson EB, Yen S, Costello E, Rosen K, Begin A, Pistorello J, \& Pearlstein T (2004). Combined dialectical behavior therapy and fluoxetine in the treatment of borderline personality disorder. J Clin Psychiatry, 65(3), 379-85.

Simpson WB (1994). Borderline personality disorder: dimension or category? A maximum covariance analysis. Dissertation abstracts international, 55, 1194.

Sinha BK, \& Watson DC (1999). Predicting personality disorder traits with the Defense Style Questionnaire in a normal sample. J Personal Disord, 13(3), 281-6.

Skodol AE, Buckley $P, \&$ Charles $E$ (1983). Is there a characteristic pattern to the treatment history of clinic outpatients with borderline personality? J Nerv Ment Dis, 171(7), 405-10.

Skodol AE, Gunderson JG, McGlashan TH, Dyck IR, Stout RL, Bender DS, Grilo CM, Shea MT, Zanarini MC, Morey LC, Sanislow CA, \& Oldham JM (2002). Functional impairment in patients with schizotypal, borderline, avoidant, or obsessive-compulsive personality disorder. Am J Psychiatry, 159(2), 276-83.

Skodol AE, \& Oldham JM (1991). Assessment and diagnosis of borderline personality disorder. Hosp Community Psychiatry, 42(10), 1021-8.

Skodol AE, Siever LJ, Livesley WJ, Gunderson JG, Pfohl B, \& Widiger TA (2002). The borderline diagnosis II: biology, genetics, and clinical course. Biol Psychiatry, 51(12), 951-63.

Sloane RB, Staples FR, Cristol AH, Yorkston NJ, \& Whipple K (1975). Psychotherapy versus behavior therapy. Cambridge MA: Harvard University Press.

Spinhoven P, van Gaalen HAE, \& Abraham RE (1995). The defense style questionnaire: a psychometric examination. J Personal Disord(9), 124-133.

Spitzer RL, Endicott J, \& Gibbon M (1979). Crossing the border into borderline personality and borderline schizophrenia. The development of criteria. Arch Gen Psychiatry, 36(1), 17-24.

Staten-Generaal TKd (vergaderjaar 1995-1996). Financieel overzicht zorg 1996. (pp. 24404 nr.2). Den Haag: Tweede kamer der staten generaal.

Steinberg M (1993). Semi-structured clinical interview for DSM-IV dissociative disorders (SCIDD). Washington DC: American Psychiatric Association.

Stern A (1938). Psychoanalytic investigation of and therapy in the borderline group of neuroses. Psychoanalytic Quarterly, 7(467-489).

Stevenson J, \& Meares R (1992). An outcome study of psychotherapy for patients with borderline personality disorder. Am J Psychiatry, 149(3), 358-62.

Stevenson J, Meares R, \& D'Angelo R (2005). Five-year outcome of outpatient psychotherapy with borderline patients. Psychol Med, 35(1), 79-87.

Stone MH (1993). Long-term outcome in personality disorders. Br J Psychiatry, 162, 299-313.

Surwillo WW (1980). Experimental design in psychiatry. Research methods for clinical practice. New York: Grune \& Stratton.

Tang TZ, \& DeRubeis RJ (1999). Sudden gains and critical sessions in cognitive-behavioral therapy for depression. J Consult Clin Psychol, 67(6), 894-904.

Ten Have ML, Lorsheyd JJG, Bijl R, \& van Osterthun P (1995). Jaarboek geestelijke gezondheidszorg 1995/1996: De GGZ in getallen. . Utrecht: De Tijdstroom.

Torgersen S, Kringlen E, \& Cramer V (2001). The prevalence of personality disorders in a community sample. Arch Gen Psychiatry, 58(6), 590-6. 
Trijsburg RW, van t' Spijker A, Van HL, Hesselink AJ, \& Duivenvoorden HJ (2000). Measuring overall defensive functioning with the Defense Style Questionnaire: a comparison of different scoring methods. J Nerv Ment Dis, 188(7), 432-9.

Trull TJ, Widiger TA, \& Guthrie P (1990). Categorical versus dimensional status of borderline personality disorder. J Abnorm Psychol, 99(1), 40-8.

Trull TJ, Widiger TA, Lynam DR, \& Costa PT, Jr. (2003). Borderline personality disorder from the perspective of general personality functioning. J Abnorm Psychol, 112(2), 193-202.

Tucker L, Bauer SF, Wagner S, Harlam D, \& Sher I (1987). Long-term hospital treatment of borderline patients: a descriptive outcome study. Am J Psychiatry, 144(11), 1443-8.

Turner RM (1989). Case study evaluations of bio-cognitive-behavioral approach for the treatment of borderline personality disorder. Behav Ther, 20, 477-489.

Turner RM, Becker L, \& DeLoach C (1994). Borderline personality. In Dattilio FM \& Freeman A (Eds.), Cognitive-behavioral strategies in crisis intervention. New York: The Guilford Press.

Tyrer $\mathrm{P}$ (2002). Practice guideline for the treatment of borderline personality disorder: a bridge too far. J Personal Disord, 16(2), 113-8.

Tyrer P, \& Johnson T (1996). Establishing the severity of personality disorder. Am J Psychiatry, 153(12), 1593-7.

Vaillant GE (1976). Natural history of male psychological health. V. The relation of choice of ego mechanisms of defense to adult adjustment. Arch Gen Psychiatry, 33(5), 535-45.

Vaillant GE (1994). Ego mechanisms of defense and personality psychopathology. J Abnorm Psychol, 103(1), 44-50.

van Asselt ADI (2006). Cost-effectiveness of schema focused therapy and transference focused psychotherapy for borderline personality disorder. Maastricht University Medical Center: Dissertation in preparation.

van Asselt ADI, Dirksen CD, Severens JL, \& Arntz A (2006). The cost of borderline personality disorder: societal cost of illness in BPD patients. Submitted for publication.

van den Hout JH, Arntz A, \& Kunkels FH (1995). Efficacy of a self-control therapy program in a psychiatric day-treatment center. Acta Psychiatr Scand, 92(1), 25-9.

van der Bossche CMR, Kremers I, Sieswerda S, \& Arntz A (1999). Vragenlijst naar belastende gebeurtenissen. Maastricht: Author.

Verheul R, van den Bosch LM, Koeter MW, de Ridder MA, Stijnen T, \& van den Brink W (2003). Dialectical behaviour therapy for women with borderline personality disorder: 12-month, randomised clinical trial in The Netherlands. Br J Psychiatry, 182, 135-40.

Waldinger RJ, \& Frank AF (1989). Clinicians' experiences in combining medication and psychotherapy in the treatment of borderline patients. Hosp Community Psychiatry, 40(7), 712-8.

Waldinger RJ, \& Gunderson JG (1984). Completed psychotherapies with borderline patients. Am J Psychother, 38(2), 190-202.

Wallner Samstag MA, Batchelder ST, Muran JC, Safran JD, \& Winston A (1998). Early identification of treatment failures in short-term psychotherapy. Journal of Psychotherapy Practice and Research, 7, 126-143.

Watson D, Clark LA, \& Harkness AR (1994). Structures of personality and their relevance to psychopathology. J Abnorm Psychol, 103(1), 18-31.

Weaver TL, \& Clum GA (1993). Early family environments and traumatic experiences associated with borderline personality disorder. J Consult Clin Psychol, 61(6), 1068-75. 
Weertman A, Arntz A, Dreessen L, van Velzen C, \& Vertommen S (2003). Short-interval testretest interrater reliability of the Dutch version of the Structured Clinical Interview for DSMIV personality disorders (SCID-II). J Personal Disord, 17(6), 562-7.

Weertman A, Arntz A, \& Kerkhofs MLM (2000). Gestructureerd klinisch interview voor DSM-IV persoonlijkheidsstoornissen (SCID-II). Lisse: Swets \& Zeitlinger.

Westen D A model and a method for uncovering the nomothetic from the idiographic: an alternative to the five-factor model? J Res Pers, 30, 400-413.

Westen D, \& Shedler J (1999). Revising and assessing axis II, Part II: toward an empirically based and clinically useful classification of personality disorders. Am J Psychiatry, 156(2), 273-85.

WHOQOL Group TW (1998). The World Health Organization Quality of Life Assessment: development and general psychometric properties. Soc Sci Med, 46(12), 1569-85.

Widiger TA, \& Frances AJ (1989). Epidemiology, diagnosis and comorbidity of borderline personality disorder. In Tasman A, Hales RE, \& Frances AJ (Eds.), American Psychiatric Press review of psychiatry (Vol. 8). Washington DC: American Psychiatric Press.

Widiger TA, \& Mullins-Sweat SN (2005). Categorical and dimensional models of personality disorders. In Oldham JM, Skodol AE, \& Bender DS (Eds.), Textbook of personality disorders. Washington DC: The American Psychiatric Publishing Inc.

Wilcox RR (1997). ANCOVA based on comparing a robust measure of location at empirically determined design points. Br J Math Stat Psychol, 50, 93-103.

Wilcox RR (2005). Introduction to robust estimation and hypothesis testing. 2nd ed. San Diego: Academic Press.

Wilcox RR (2006). ANCOVA: A robust omnibus test based on selected design points. J Mod Appl Stat Methods, In Press.

Wildgoose A, Clarke S, \& Waller G (2001). Treating personality fragmentation and dissociation in borderline personality disorder: A pilot study of the impact of cognitive analytic therapy. $\mathrm{Br}$ J Med Psychol, 74 Part 1, 47-55.

Yeomans F, Selzer M, \& Clarkin J (1993). Studying the treatment contract in intensive psychotherapy with borderline patients. Psychiatry, 56(3), 254-63; discussion 264-9.

Yeomans FE, Clarkin JF, \& Kernberg OF (2002). A primer for transference focused psychotherapy for the borderline patient. Northvale NJ: Jason Aronson.

Yeomans FE, Selzer MA, \& Clarkin JF (1992). Treating the borderline patient: a contract-based approach. New York: Basic Books.

Young J, Arntz A, \& Giesen-Bloo J (1999). Therapy adherence and competence scale for schema focused therapy. New York/Maastricht: Authors.

Young JE (1999). Cognitive therapy for personality disorders: a schema-focused approach. (Revised edition ed.). Sarasota FL: Professional Resource Press.

Young JE, \& Klosko J (1994). Reinventing your life. New York: Plume.

Young JE, Klosko J, \& Weishaar ME (2003). Schema therapy: a practitioner's guide. New York: The Guilford Press.

Zanarini MC, Frankenburg FR, Dubo ED, Sickel AE, Trikha A, Levin A, \& Reynolds V (1998a). Axis I comorbidity of borderline personality disorder. Am J Psychiatry, 155(12), 1733-9.

Zanarini MC, Frankenburg FR, Dubo ED, Sickel AE, Trikha A, Levin A, \& Reynolds V (1998b). Axis II comorbidity of borderline personality disorder. Compr Psychiatry, 39(5), 296-302. 
Zanarini MC, Frankenburg FR, Hennen J, \& Silk KR (2003). The longitudinal course of borderline psychopathology: 6-year prospective follow-up of the phenomenology of borderline personality disorder. Am J Psychiatry, 160(2), 274-83.

Zanarini MC, Frankenburg FR, Hennen J, \& Silk KR (2004). Mental health service utilization by borderline personality disorder patients and Axis II comparison subjects followed prospectively for 6 years. J Clin Psychiatry, 65(1), 28-36.

Zanarini MC, Gunderson J, Frankenburg FR, \& Chauncey DL (1989). The revised diagnostic interview for borderlines: discriminating BPD from other axis II disorders. J Personal Disord, 3, 10-18.

Zimmerman I, Woo S, Glasser JM, \& Alan J (1973). Clinical interpretation of the Wechsler Adult Intelligence Scale. Oxford: Grune \& Stratton.

Zimmerman M (1994). Diagnosing personality disorders. A review of issues and research methods. Arch Gen Psychiatry, 51(3), 225-45.

Zimmerman M, \& Coryell WH (1990). Diagnosing personality disorders in the community. A comparison of self-report and interview measures. Arch Gen Psychiatry, 47(6), 527-31.

Zimmerman M, \& Mattia Jl (1999a). Differences between clinical and research practices in diagnosing borderline personality disorder. Am J Psychiatry, 156(10), 1570-4.

Zimmerman M, \& Mattia Jl (1999b). Psychiatric diagnosis in clinical practice: is comorbidity being missed? Compr Psychiatry, 40(3), 182-91. 


\section{Dutch Summary/ \\ Nederlandse samenvatting}




\section{Nederlandse samenvatting}

Het onderwerp van dit proefschrift is de Borderline Persoonlijkheidsstoornis (BPS). Cliënten met een BPS hebben veelal te maken met complexe problematiek, waarin instabiliteit eigenlijk het enige stabiele kenmerk lijkt te zijn. Dat geldt in eerste instantie voor henzelf, maar ook voor de mensen om hem/haar heen zoals partners, familie, vrienden of collega's, en voor de mensen die met borderline cliënten te maken hebben via hun werk in de (geestelijke) gezondheidszorg of andere sociale diensten. Deel I van dit proefschrift geeft aandacht aan de historische ontwikkeling van het borderline concept. In dit proefschrift wordt uitgegaan van de borderline conceptualisering zoals beschreven in de Diagnostic and Statistical Manual of Mental Disorders, fourth edition (DSM-IV). Vervolgens is ingegaan op enkele karakteristieken, etiologische aspecten, meetmethoden voor BPSproblematiek en de stand van zaken met betrekking tot de behandeling van BPS beschreven. Daarnaast zijn 'op overdracht gerichte psychoanalytische psychotherapie' (transference focused psychotherapy, TFP) en 'schemagerichte cognitieve gedragstherapie' (schema focused therapy, SFT) in meer detail beschreven. De doelstellingen van dit proefschrift hadden betrekking op (1) het conceptuele debat rondom de BPS, (2) het ontwikkelen van betrouwbare, valide en verandering - gevoelige BPS-specifieke meetinstrumenten en (3) de effectiviteit van langdurige therapie (TFP en SFT) voor cliënten met BPS.

\section{Theorie}

Deel II bevat twee studies waarin theoretische issues van BPS centraal staan. Het doel van hoofdstuk 2 was te onderzoeken of BPS wordt gekenmerkt door specifieke wereldbeeld assumpties welke zijn afgeleid van de cognitieve theorie die zegt dat BPS wordt gekenmerkt door drie basisassumpties (De wereld is, c.q. anderen zijn, kwaadaardig en gevaarlijk; ik ben machteloos en kwetsbaar; ik ben slecht). Dit is getoetst aan de hand van (Janoff-Bulman, 1989) wereld assumptie model met betrekking tot de negatieve effecten van trauma. Daarnaast is de rol van trauma in de wereld assumpties van BPS-cliënten nader onderzocht. Hiertoe hebben BPS-cliënten, cliënten met Cluster $C$ persoonlijkheidsstoornissen, cliënten met enkel as-I psychopathologie en klachtvrije controle personen verschillende vragenlijsten ingevuld. BPS-cliënten zien de wereld als kwaadaardig en ervaren minder 'geluk' onafhankelijk van de mate van ervaren trauma, maar afhankelijk van de ervaren ernst van BPS-psychopathologie. Daarnaast hebben BPS-cliënten weinig 'zelfwaarde' en blijven vasthouden aan bepaalde assumpties onafhankelijk van ervaren trauma of ervaren ernst van de BPS-psychopathologie. Daarmee wordt de cognitieve theorie grotendeels ondersteund door middel van Janoff-Bulman's wereld assumptie model. Verder kan worden geconcludeerd dat 
de wereldassumpties van BPS-cliënten in deze studie beter kunnen worden verklaard door de ernst van de BPD-psychopathologie dan door de aanwezigheid van trauma.

Hoofdstuk 3 richt zich op het gebruik van afweermechanismen volgens moderne psychoanalytici met behulp van de 'Defense Style Questionnaire - 48' (DSQ-48). De doelen van deze studie waren het repliceren van de 3-factor structuur van de DSQ-36 (Spinhoven, van Gaalen, et al., 1995), een psychometrische evaluatie van de DSQ-48 en het vergelijken van de afweerstijl van verschillende cliënt- en controle groepen. Dezelfde groepen mensen als in de eerste studie werkten mee aan deza studie. Valideringsdata werden verkregen met zelfrapportage vragenlijsten en een semi-gestructureerd interview. De bevindingen van $\mathrm{Sp}$ inhoven et al. werden inderdaad gerepliceerd, echter ook met dezelfde psychometrische gebreken. Een daaropvolgende exploratieve principale componenten analyse liet een 5-factor structuur (wantrouwen, zelfbescherming, zelfopoffering, vermijding en controle) zien met betere psychometrische eigenschappen. Een replicatie van de 5 -factor structuur in een nieuwe groep klachtvrije controle personen gaf bevredigende resultaten.

\section{Meten}

Deel III omvat twee studies die zich richtten op het meten van BPSpsychopathologie en het concept BPS. De hoofdstukken 4 en 5 onderzochten BPD specifieke meetinstrumenten: een zelfrapportage vragenlijst 'de BPD Checklist' en een semigestructureerd interview 'de Borderline Personality Disorder Severity Index - IV (BPDSI-IV)'. Deze instrumenten zijn ontwikkeld om een hiaat van BPD-specifieke meetinstrumenten te vullen, welke de aard en de complexiteit van BPS vereisen. Beide instrumenten verkrijgen gedetailleerde informatie met betrekking tot de ernst en de frequentie van BPS-specifieke pathologie en zijn ontwikkeld om als behandeluitkomstmaat te functioneren. De BPD Checklist meet echter de last die een cliënt ervaart van zijn/haar BPS symptomen, terwijl de BPDSI-IV de werkelijke BPS-manifestaties objectief vastlegt. Opnieuw vormden BPS-cliënten, cliënten met cluster $C$ persoonlijkheidspathologie, cliënten met as I pathologie en klachtvrije controle personen de studie populaties. De psychometrische eigenschappen van beide instrumenten waren zeer goed. Tevens werden klinische normen, met bijbehorende specificiteit en sensitiviteit, vastgesteld. De gevoeligheid tot verandering door behandeling werd alleen onderzocht voor de BPD Checklist en met positief resultaat. De grote gevoeligheid voor verandering door behandeling voor de BPDSI-IV wordt wel aangetoond in hoofdstuk 6. Een andere belangrijke doelstelling in zowel hoofdstuk 4 als 5 had betrekking op zeven dimensionele modellen van BPS. Deze modellen werden gebruikt om te zien of de BPD Checklist en BPDSI-IV data met gebruikmaking van eerste orde 
confirmerende factor analyses een één, een hogere orde of een multidimensioneel BPS-concept het best ondersteund. Opvallend was dat zowel de BPD Checklist items als de BPDSI-IV items laten zien dat een 1- én een 9- (gebaseerd op de DSM-IV BPS criteria) factor model van BPS het beste wordt verklaard door de data.

\section{Behandeling}

In deel IV worden studies gerapporteerd waarin de behandeling van BPScliënten centraal staat. Hoofdstuk 6 gaat over een multicenter gerandomiseerd onderzoek waarin de effectiviteit van SFT en TFP met elkaar worden vergeleken. 88 Cliënten met BPS van vier reguliere GGZ-instellingen in Nederland namen deel aan de studie. Gedurende drie jaar werden zij gevolgd tijdens hun SFT of TFP behandeling (twee sessies per week). De onderzoeksmetingen van cliënten vonden plaats vlak voor de randomisatie en vervolgens iedere drie maanden gedurende drie jaar. De belangrijkste uitkomstmaten waren de BPDSI-IV om de verandering in BPS-specifieke pathologie vast te stellen, de ervaren kwaliteit van leven (indirect en direct) en meetinstrumenten die de onderliggende concepten van SFT en TFP in kaart brengen. De gegevens van 44 SFT- en 42 TFP cliënten werden geanalyseerd. Beide groepen waren vergelijkbaar wat betreft de sociodemografische en klinische karakteristieken. Een eerste bevinding was dat TFP-cliënten een hogere kans op voortijdige behandeluitval hebben dan SFT-cliënten. Beide behandelingen lieten statistisch en klinisch significante verbeteringen zien op alle uitkomstmaten na 1-, 2- en 3-jaar behandeling. Daarnaast bleek dat, op grond van de BPDSI-IV, na 3 jaar behandeling significant meer SFT-cliënten dan TFPcliënten het 'beter'-criterium en het 'reliable change'-criterium hadden bereikt. Ook bleken SFT-cliënten significant meer te zijn verbeterd voor wat betreft algemene psychopathologisch disfunctioneren en de maten van SFT/TFP persoonlijkheidsconcepten. De toename in ervaren kwaliteit van leven was voor SFTcliënten eveneens significant groter dan voor TFP-cliënten. Al met al kon worden geconcludeerd dat SFT in deze studie effectiever was dan TFP. In hoofdstuk 7 wordt de stand van zaken van de clientengroepen uit de RCT na een follow-up periode van 1 jaar besproken. Daarin werd vastgesteld dat de gevonden verbeteringen voor TFP en SFT na 3 jaar behandeling op alle uitkomstmaten voortduurden. De verschillen in verbetering tussen TFP en SFT bleven ook bestaan. Echter de additionele verbeteringen tijdens het follow-up jaar waren niet significant. De behandelstudie draagt in belangrijke mate bij aan een positief behandelperspectief van BPS-problematiek.

Hoofdstuk 8 belicht een andere zijde van het eerdergenoemde behandelonderzoek en gaat in op de kwaliteit en ontwikkeling van de therapeutische alliantie als mediator in het SFT- en TFP-behandelproces c.q. genezingsproces. 78 van de 
cliënten werden in dit onderzoek betrokken. De waardering van de therapeutische alliantie was voor zowel de SFT-cliënten als de SFT-therapeuten significant groter dan voor TFP-cliënten en -therapeuten. Negatieve beoordelingen van clienten en therapeuten in het begin van de behandeling bleken voortijdige behandeluitval te voorspellen, terwijl toenemende positieve beoordelingen van cliënten in de eerste helft van de therapie in staat waren om verdere klinische verbetering te voorspellen. Verder werd duidelijk dat verschillen in pathologische persoonlijkheidskenmerken tussen therapeuten en cliënten rechtstreeks van invloed was op de groei van de therapeutische alliantie. De therapeutische alliantie is dus een belangrijke gemeenschappelijke therapiefactor die wordt beinvloed door het type behandeling.

In Deel $\mathrm{V}$ van het proefschrift worden de belangrijkste conclusies van het gepresenteerde onderzoek nog eens op een rij gezet. Vervolgens worden de sterke en zwakke kanten van de borderline behandelstudie benoemd, en enkele opvallende observaties verder besproken. Daarnaast wordt de rol van zogenaamde patient-therapeut factoren verder uitgewerkt. Een ander punt van aandacht in de discussie vormt de BPS- conceptualisatie; de studies van dit proefschrift worden geplaatst in het debat over categoriële tegenover dimensionele classificaties van BPS. Verder wordt ingegaan op vragen rondom het meten van, en meetinstrumenten voor, BPS. De klinische implicaties en toekomstig onderzoek worden eveneens besproken.

Tot slot. In dit proefschrift zijn diverse grenzen gepasseerd of verlegd (crossing borders). Allereerst zijn (delen van) psychologische theorieën ter verklaring van BPS getoetst aan de hand van andere, verwante psychologische theorieën; Beck's cognitieve theorie voor BPS werd getoetst aan Janoff-Bulman's sociaal cognitieve wereld assumpties en psychodynamische afweerstijlen werden getoetst aan object-relatie gebaseerde primitieve afweer- en cognitieve coping mechanismen. Een tweede grens had betrekking op de ontwikkeling van betrouwbare, valide en verandering-gevoelige meetinstrumenten (de BPDSI-IV en de BPDChecklist), specifiek voor het vaststellen van BPS-pathologie in screening- en behandeleffect procedures. Dit heeft ook betrekking op de verschillende formats, zelfrapportage en semigestructureerd interview, die zowel subjectieve als objectieve metingen mogelijk maken. De derde grens slaat op de geslaagde uitvoering van een methodologisch degelijk opgezet 'randomized clinical trial' van ambulante psychotherapieën (SFT en TFP) voor cliënten met BPS, die bijdraagt aan het veranderend behandelperspectief van BPS-problematiek, van onbehandelbaar naar behandelbaar. Tenslotte houdt een vierde grens verband met de vooralsnog unieke samenwerking van twee verschillende psychologische 'scholen' in één onderzoek. 



\section{Appendices}





\title{
Appendix I
}

\author{
Pilot Study \\ "Interpersonal behavior style of SFT and TFP therapists"
}

Background. The SFT and TFP manuals elaborate on the therapeutic position, as well as how and when to deviate from, and return to, this position in the therapy process (see chapter 1). Leary's interpersonal model (1957) can be used in describing and explaining interaction between persons. In this model, interpersonal behavioral styles can be ranked across two square axes: dominance and affiliation. The vertical axis 'dominance' is marked by control or dominant behavior on top and submissive, obedient behavior on the bottom. The horizontal axis 'affiliation' is characterized by hostility, or 'against others' on the left end, and by friendliness, or collaboration on the right end. Within the interpersonal model, it can be hypothesized that the TFP-manual appears to prescribe an interpersonal behavioral style for the TFP-therapist that is largely determined by dimensions reflected in the 'dominance'-axis, and much less by those of the 'affiliation'-axis. The hypothesis for the SFT-protocol is that dominance and affiliation dimensions are equally represented in defining the interpersonal style of a SFT-therapist.

Method. From 16 SFT- and 16 TFP therapists, and their corresponding borderline disordered patients, the first six audio tapes were used to obtain observer-rated assessments of interpersonal behavior style with the Interpersonal Checklist - Revised version (ICL-R; de Jong, van den Brink, \& Jansma, 2000; Laforge \& Suczek, 1955; Paddock \& Nowicki, 1986) and the Interpersonal Behavior Observation (IBO; de Jong, 1998). The IBO and ICL-R are both based on the interpersonal behavioral model. The dominance- and affiliation-scores are determined by ten behavioral styles (subscales) which have a circumplex structure around the two axes. The IBO consists of two visual analogue scales, one for dominance and one for affiliation. The ICL-R questionnaire consists of 160 yes/no-items (16 items per style), designed in a manner that enables a respondent to rate him/herself or another person. The therapists did not differ with respect to gender, professional status, years of clinical experience. Therapists did differ for age, for which analyses were corrected. Six independent graduate students in Psychology/Mental Health Sciences (University Maastricht) rated sets of tapes in random order. IBO ratings were made after each tape for both therapist and patient. Observer ICL-R ratings were made for both therapist and patient after all tapes were listened to. The raters also filled out an ICL-R for their own interpersonal behavioral style, as these scores were covariates. Two-sided t-tests were used in comparing SFT- and TFP-therapists with respect to their mean ICL$\mathrm{R}$ and IBO affiliation and dominance levels. Then, multiple regression tested which variables (treatment condition, patient and therapist affiliation and domi- 
nance scores, baseline BPDSI-IV) significantly influenced affiliation and dominance ratings.

Results and comment. Table 1 contains mean IBO and ICL-R affiliation and dominance scores of SFT- and TFP-therapists and test statistics. Only IBOaffiliation scores were significantly different, meaning that SFT-therapists were warmer, friendlier and more 'together' with the patient than TFP-therapists. The subsequent multiple regression analyses also revealed a single relevant effect; treatment condition was of significant influence on therapists' IBO-affiliation scores $(\beta=0.45, P=.018)$.

Table 1 Mean IBO and ICL-R affiliation and dominance scores of SFT- and TFP-therapists and test statistics.

\begin{tabular}{lclccc}
\hline & $\begin{array}{c}\text { SFT-Therapists } \\
\text { mean (sd) }\end{array}$ & $\begin{array}{c}\text { TFP-Therapists } \\
\text { mean (sd) }\end{array}$ & T & df & $\boldsymbol{P}$-value \\
\hline IBO & & & & & \\
Dominance & $0.41(0.37)$ & $0.30(0.31)$ & -0.90 & 30 & .380 \\
Affiliation & $1.19(0.35)$ & $0.88(0.36)$ & -2.50 & 30 & .018 \\
ICL-R & & & & & \\
Dominance & $10.54(7.95)$ & $5.74(7.03)$ & -1.73 & 30 & .094 \\
Affiliation & $6.66(4.19)$ & $1.84(8.53)$ & -1.41 & 30 & .169 \\
\hline
\end{tabular}

Note: Two-sided T-tests.

The inter-rater reliability of the IBO- and ICL-R ratings were very disappointing (ICC's; IBO-affiliation 0.21; IBO-dominance 0.19; ICL-R affiliation 0.17), except for ICL-R dominance (ICC 0.89). So, even though the IBO-affiliation scores seemed to provide some support for our hypothesis, not much weight can be given to this finding. If anything, it can be concluded that the formulated hypotheses cannot be accepted or rejected but should be tested with different instruments and/or other fundamental adjustments to the design (e.g. larger $n$; self-report ICL-R for patients and therapists; patients and therapists rating the ICL-R over each other). 


\section{Appendix II}

\section{BPDSI-IV Criterion cutoff scores}

Extremely skewed distributions led to the calculation of nonparametric estimates in addition to Jacobson \& Truax's parametric procedures (1991). Since a nonparametric approach can also yield unreliable estimates of scores distinguishing non-patients from BPD-patients, by using individual observations and not a theoretical distribution, several nonparametric procedures were employed. For one, criteria cutoff scores were estimated with ROC curve analysis i.e. the empirical point that maximizes sensitivity and specificity between BPD- and nonpatients. Points halfway the upper $75^{\text {th }}$ percentile of the non-patient sample and the lower $25^{\text {th }}$ percentile of the BPD sample were calculated for each criterion, as well as for the percentile ratios $80 / 20,90 / 10,95 / 5$ and $97.5 / 2.5$. If scores were normally distributed, the Jacobson and Truax's a-criterion (i.e. 2 standard deviations above the non-patients mean) would be the same as the 97.5 percentile of the observed distribution, but this was not the case. All calculated nonparametric estimates had high sensitivity and specificity, and were near the maximum value of the ROC-analyses. Specificity was generally higher than sensitivity, which is acceptable given the fact that these norms were calculated to detect treatment change towards recovery and not towards a BPD-diagnosis. As final cutoff scores we looked for round figures to facilitate use in clinical practice, these cutoff scores are depicted in Table 2.

Table 2. BPDSI-IV criteria cutoff scores, specificity and sensitivity

\begin{tabular}{llll}
\hline Criterion & Cutoff score & specificity & sensitivity \\
\hline 1 abandonment & 1 & .974 & .852 \\
2 relationships & 1 & .987 & .935 \\
3 identity & 1 & 1.000 & .972 \\
4 impulsivity & .5 & .921 & .870 \\
5 parasuicide & .1 & .987 & .787 \\
6 mood & 2 & .987 & .963 \\
7 emptiness & 2 & .987 & .972 \\
8 anger & 1 & .895 & .861 \\
9 dissociation & .3 & .987 & .917 \\
\hline
\end{tabular}

In a next step, the cutoff scores obtained were used to verify whether a diagnosis of BPD was met or not (i.e. like the DSM-IV, meeting 5 BPDSI-IV criteria qualified for a BPD-diagnosis). All but one BPD-patient were correctly identified (sensitivity $=0.991$ ), this one patient was also an anomaly to the BPDSI-IV total score (9.85). Specificity was perfect with respect to non-patients (1.000); satisfactory with respect to axis-I patients $(0.889)$; and moderate with respect 
to Cluster-C personality disordered patients (0.667). The latter score can be understood since comorbidity of Cluster $\mathrm{C}$ personality disorders in BPD-patients is quite common (Oldham, Skodol, et al., 1995; Zanarini, Frankenburg, et al., 1998b). 


\section{Appendix III}

\section{The Defense Style Questionnaire - 48}

Naam (optioneel):

Geslacht:

Leeftijd:

Hoogst voltooide opleiding:

( 1 =geen, 2 =buitengewoon lager onderwijs, $3=$ lagere school/basisonderwijs, $4=$ lager voortgezet onderwijs, $5=\mathrm{lbo}, 6=\mathrm{mvo}$ (mulo, mavo, ivo) $7=$ hvo (havo, $\mathrm{mms}$ ), $8=\mathrm{mbo}, 9=$ vwo, $10=$ hbo, $11=$ wo)

Deze vragenlijst bestaat uit 48 uitspraken, geef achter elke uitspraak aan in welke mate $u$ het er mee eens bent door een getal tussen de 1 en de 9 achter de uitspraak aan te geven.

$\begin{array}{lllllllllll}\text { zeer mee oneens } & 1 & 2 & 3 & 4 & 5 & 6 & 7 & 8 & 9 & \text { zeer mee eens }\end{array}$

Voorbeeld:

1. Ik houd van rozen

wanneer u het er "zeer mee eens" bent, dan zet u een 9 achter de uitspraak:

1. Ik houd van rozen

9

\begin{tabular}{lllllllllll}
\hline zeer mee oneens & 1 & 2 & 3 & 4 & 5 & 6 & 7 & 8 & 9 & zeer mee eens \\
\hline
\end{tabular}

1. Men vindt mij vaak een chagrijnig persoon

2. Ik kan een probleem uit m'n hoofd zetten, totdat ik tijd heb om er wat aan te doen.

3. Ik word altijd oneerlijk behandeld

4. Ik reageer mijn onrust af door iets praktisch en creatiefs te doen zoals schilderen of hout bewerken.

5. Ik raak altijd verzeild in dezelfde soort vervelende situaties en ik weet niet waarom.

6. Ik kan gemakkelijk om mezelf lachen.

7. Ik reageer als een kind als ik gefrustreerd ben.

8. Men is geneigd mij slecht te behandelen. 


\begin{tabular}{lllllllllll}
\hline zeer mee oneens & 1 & 2 & 3 & 4 & 5 & 6 & 7 & 8 & 9 & zeer mee eens
\end{tabular}

9. Men vindt dat ik een struisvogel ben met $m^{\prime} n$ kop in het zand. Met andere woorden: ik ben geneigd vervelende dingen te negeren door te doen alsof ze niet bestaan.

10. Gevoelsmatig is er iemand die me alles afneemt wat ik van waarde vind.

11. Ik ben trots op mijn eigenschap mensen op hun plaats te zetten.

12. Men zegt dat ik een achtervolgingscomplex heb.

13. Ik word lichamelijk ziek als dingen niet goed voor mij gaan.

14. Ik ben een heel geremd persoon.

15 Mijn vrienden zien mij als een clown.

16 Ik werk meer dingen uit in mijn dagdromen dan in mijn feitelijk leven.

17. Ik ben zeer verlegen als het gaat om het benaderen van mensen.

18. Ik ben nergens bang voor.

19. Ik heb altijd het gevoel dat iemand die ik ken een soort beschermengel voor $\mathrm{mij}$ is.

20. Wat mij betreft zijn mensen ofwel goed, ofwel slecht.

21. ledereen is tegen mij.

22. Als mijn gevoelens mij zouden belemmeren in waar ik mee bezig ben, als ik ze de vrije loop zou laten, dan kan ik mezelf voor deze gevoelens afsluiten.

23. Sommige mensen zweren tegen mij samen.

24. Ik ben gewoonlijk in staat om de humoristische kant in te zien van een zaak die anders pijnlijk zou zijn.

25. Ik krijg hoofdpijn als ik dingen moet doen die ik niet leuk vind.

26. Als je slecht bent, ben je helemaal slecht. “lets goeds vinden in iedereen" bestaat niet.

27. We zouden nooit boos moeten worden op mensen die we onaardig vinden.

28. Als ik weet dat ik een moeilijke situatie onder ogen moet zien, zoals een examen of een sollicitatiegesprek, probeer ik mij van te voren in te beelden hoe het zal zijn en probeer ik manieren te bedenken om er mee om te gaan.

29. Als iemand in mijn naaste omgeving sterft, voel ik mij 


\begin{tabular}{lllllllllll}
\hline zeer mee oneens & 1 & 2 & 3 & 4 & 5 & 6 & 7 & 8 & 9 & zeer mee eens \\
\hline
\end{tabular}

niet geschokt.

30. Nadat ik voor mijn rechten heb gevochten, ben ik geneigd mij te verontschuldigen voor het feit dat ik voor mijzelf ben opgekomen.

31. Ik voel mij beter door hard te werken.

32. Ik heb gewoonten of gebruiken die ik wel moet uitvoeren, omdat er anders iets verschrikkelijks zal gebeuren.

33. Als ik kan voorspellen dat ik binnenkort treurig zal worden, kan ik er beter mee om gaan.

34. Vaak merk ik dat ik niets voel, terwijl de situatie aanleiding zou moeten geven tot sterke gevoelens.

35. Door bezig te blijven met de taak die ik uitvoer, word ik afgehouden van sombere en onrustige gevoelens.

36. Als ik een agressieve gedachte heb, voel ik de drang om iets te doen dat dit goed maakt.

37. Ik vind het prettig om anderen te helpen en als ik dat niet meer zou kunnen doen, zou ik me naar voelen.

38. Ik ben heel verlegen als ik tegenover anderen voor $\mathrm{m}^{\prime} \mathrm{n}$ rechten op moet komen.

39. Als iemand mij in elkaar slaat en m'n geld rooft, heb ik liever dat ze hem helpen dan mij.

40. Ik val stil als ze gaan doen wie ergens het beste in is.

41. Als ik me gekwetst voel, trek ik me in m'n schulp terug.

42. Ik vind het een goede zaak om iemand die me kwetst, m'n andere wang toe te keren.

43. Ik wil met het kwaad niets maar dan ook niets te maken hebben.

44. Ik probeer aardig te zijn tegen mensen die ik niet mag.

45. Ik ken iemand die alles kan en volstrekt eerlijk en rechtvaardig is.

46. Vaak ben ik erg aardig voor mensen waarop ik terecht boos zou moeten zijn.

47. Als ik me naar voel, zoek ik gezelschap.

48. Als ik het ergens erg moeilijk mee heb, ga ik iemand opzoeken die hetzelfde probleem heeft gehad. 


\section{The Defense Style Questionnaire - 43}

Items 47 and 48 of the DSQ-48 had insufficient factor loadings and were therefore not included in the DSO-43.

Items 13,25 and 45 of the DSQ-43 did not match any of the new five factors' content and were therefore not included in the DSQ-43. 


\section{The Borderline Personality Disorder Severity Index - IV}

\section{Versie IV}

Bewerking naar Weaver \& Clum, 1993

Maastricht, 06/09/1995, 04/04/1996, 11/11/1998, 08/07/1999

Arnoud Arntz en Josephine Giesen-Bloo

Departement Medische, Klinische en Experimentele Psychologie, Universiteit Maastricht.

Met dank aan: Mw. M.Burger, Mw. drs. I. Callewaerd, Mw. drs. A.E.F. Cleven, Mw. drs. M. van den Hoorn, Mw. H.Keijsers, Mw. drs. E. A. Riedijk, Mw. drs. A.M. Turksma, Dhr. drs W.M. van der Vlist.

\section{Instructie}

Dit is een semi-gestructureerd klinisch interview naar de ernst van kenmerken van Borderline Persoonlijkheidsstoornis gedurende een afgebakende periode, in dit geval drie maanden. Het interview dient te worden afgenomen door een klinisch geschoolde interviewer, omdat een klinisch oordeel voor de scoring noodzakelijk is. Het interview is geschikt voor herhaalde afname, maar dient dan zoveel mogelijk door dezelfde interviewer te worden afgenomen. Om tot een afgewogen beoordeling te komen dienen naast de standaardvragen aanvullende vragen gesteld te worden. Het vragen naar concrete voorvallen/voorbeelden is noodzakelijk. Ook uitsluitingen (bijv. depersonalisatie door druggebruik) moeten actief worden uitgevraagd. In veel gevallen is het belangrijk om alle concrete voorvallen gedurende de afgelopen 3 maanden te laten beschrijven, om zo de frequentie vast te stellen. Alleen bij hoogfrequente gedragingen kan volstaan worden met enkele prototypische voorbeelden, waarna de frequentie geschat kan worden. Alle frequentievragen worden op 10-puntsschalen gescoord $(0=$ nooit; $10=$ dagelijks), ook die waar een zo hoge frequentie onwaarschijnlijk is. Bij elke onduidelijkheid dienen aantekeningen gemaakt te worden, zodat geen informatie verloren gaat. Scores en aantekeningen worden op een apart scoringsformulier gemaakt. 
Inleiding

"Dit interview gaat over een aantal zaken die mensen kunnen meemaken. De bedoeling is dat $u$ bij $u$ zelf nagaat of $u$ dat wat gevraagd wordt de afgelopen 3 maanden heeft meegemaakt, en hoe vaak dat is gebeurd. De vragen worden steeds op een vaste manier gesteld, maar als $u$ ze niet goed begrijpt kunt $u$ gerust uitleg vragen. Heeft u daar nog vragen over?

Omdat het alleen over de afgelopen 3 maanden gaat is het handig om even vast te stellen welke periode dat was. $\mathrm{Nu}$ is het (..(datum), 3 maanden geleden was het ... Dat was ongeveer (belangrijke gebeurtenis/dag)"

N.B. Alles wat schuin gedrukt is, is tekst voor de interviewer, dit dient niet hardop voorgelezen te worden.

Cliënten kunnen desgewenst met "je" aangesproken worden. 


\section{Verlating}

Deze items slaan op verwoede pogingen door de persoon in kwestie ondernomen met als doel te voorkomen dat iemand waar hij / zij een verhouding mee heeft, verbonden mee is of afhankelijk van is hem / haar gaat verlaten. Voorbeelden zijn o.a. smeken dat iemand niet zal weggaan of iemand fysiek proberen tegen te houden.

0. nooit

1. 1 x p. 3 maanden

2. $2 \times$ p. 3 maanden

3. $3 \times$ p. 3 maanden $/ 1 \times$ p. maand

4. 4 à $5 \times$ p. 3 maanden $/ 1 \times$ p. 3 weken

5. 6 à $7 \times$ p. 3 maanden $/ 1 \times$ p. 2 weken
6. 8 à $10 \times$ p. 3 maanden $/ 2 \times$ p. 3 weken
7. $1 \times$ p. week/11 à $15 \times$ p. 3 maanden
8. meerdere malen $p$. week maar minder dan de helft van de week
9. meer dan de helft van de week/bijna elke dag 10. dagelijks

1.1 Werd $\mathrm{u}$ er de afgelopen 3 maanden weleens wanhopig van als $\mathrm{u}$ dacht dat iemand waar $u$ om gaf $u$ zou gaan verlaten?

(bij positief scoren zijn duidelijke voorbeelden nodig)

1.2 Heeft $u$ de afgelopen 3 maanden iemand die belangrijk voor $u$ was en die bij $\mathrm{u}$ weg wilde (of waarvan $\mathrm{u}$ dacht dat die weg wilde) op een fanatieke manier bij u proberen te houden? (bv. continu bellen, controleren, verleiden e.d., alleen overdreven, krampachtige manieren scoren)

(het gaat om echte en ingebeelde verlatingen; bij positief scoren zijn duidelijke voorbeelden nodig van de pogingen) (Ook scoren wat bij 1.3, 1.4 en 1.5 terugkeert).

1.3 Kwam het de afgelopen 3 maanden weleens voor dat u smeekte en huilde dat iemand $\mathrm{u}$ niet moest verlaten?

1.4 Kwam het de afgelopen 3 maanden weleens voor dat $\mathrm{u}$ dreigde iets te doen, om er voor te zorgen dat iemand u niet zou verlaten? lbv. chantage, leugens, moord, zelfmoord)

1.5 Heeft $u$ de afgelopen 3 maanden ooit geprobeerd op een fysieke manier iemand tegen te houden als die $\mathrm{u}$ wilde verlaten. (bv. voor de deur gaan staan, vasthouden) 
1.6 Hoe vaak heeft $u$ de afgelopen 3 maanden een sterk verlangen gehad om te horen dat iemand van $\mathrm{u}$ hield/ om u geeft/ u niet verlaat/ $\mathrm{u}$ aantrekkelijk vond etc.? (kan zowel bij vrienden, familie als partners zijn)

1.7 Hoe vaak heeft $u$ de afgelopen 3 maanden bij andere mensen om bevestiging gevraagd, het gaat hierbij om bevestiging om $u$ gerust te stellen dat iemand u niet zal gaan verlaten.

(bij vermijding, doorvragen) Werd $\mathrm{u}$ de afgelopen 3 maanden weleens gezegd dat $u$ teveel bevestiging vraagt? 


\section{Relaties}

Er zijn drie kenmerken van dit criterium. Ten eerste moet er een patroon zijn van instabiele relaties, welke gekenmerkt worden door regelmatige conflicten en dreigende of daadwerkelijke relatiebreuk. Ten tweede, deze relaties moeten intens zijn, in die zin dat sterke emoties aanwezig zijn (bv. euforie, afkeer, woede, wrok, wanhoop). Tenslotte moet de persoon de andere persoon op sommige momenten devalueren ("hij is echt gemeen"). Op andere momtenten zou de persoon de andere kunnen idealiseren ("mijn vriend is de meest geweldige, attente en sterkste persoon die ik ooit ontmoet heb").

In analytische termen gebruiken deze personen splitting als afweermechanisme.

0. Nooit

1. $1 \times$ p. 3 maanden

2. $2 \times$ p. 3 maanden

3. $3 \times$ p. 3 maanden $/ 1 \times$ p. maand

4. 4 à $5 \times$ p. 3 maanden $/ 1 \times$ p. 3 weken

5. 6 à $7 \times$ p. 3 maanden $/ 1 \times$ p. 2 weken
6. 8 à $10 \times$ p. 3 maanden $/ 2 \times$ p. 3 weken

7. $1 \times \mathrm{p}$. week/11 à $15 \times \mathrm{p} .3$ maanden

8. meerdere malen p. week maar minder dan de helft van de week

9. meer dan de helft van de week/bijna elke dag 10. dagelijks

\section{Partnerrelatie}

2.1 Waren er momenten waarop $u$ vond dat uw partner alles was wat u wilde en andere momenten dat $\mathrm{u}$ vond dat hij/zij verschrikkelijk was? (het gaat hierbij om een overtuiging d.w.z. intrapsychisch, dus niet persé om de daadwerkelijke relatie)

2.2 Hoe vaak heeft $u$ in de afgelopen 3 maanden ups en downs in uw partnerrelatie gekend? (het gaat hierbij dus om de daadwerkelijke relatie)

2.3 Heeft u de afgelopen 3 maanden uw partnerrelatie verbroken en weer aangegaan? (zowel 'aan' als 'uit ' scoren, dus twee keer 'aan' en 'uit 'gemaakt is een 4 scoren)

2.4 Bent $u$ de afgelopen 3 maanden een of meerdere nieuwe partnerrelatie(s) aangegaan en/of heeft u die beëindigd? (zowel 'aan' als 'uit' afzonderlijk scoren)

\section{Andere relaties}

2.5 Waren er momenten waarop u vond dat uw vrienden/familieleden/collega's en/of andere belangrijke bekenden alles waren wat $u$ wilde en andere mo- 
menten dat u vond dat hij/zij verschrikkelijk waren? (het gaat hierbij om een overtuiging, dus niet persé om de daadwerkelijke relatie)

2.6 Hoe vaak heeft $u$ de afgelopen 3 maanden ups en downs in uw relaties met vrienden/familie/collega's en/of andere belangrijke bekenden gekend? (het gaat hierbij dus om de daadwerkelijke relatie)

2.7 Heeft u de afgelopen 3 maanden uw relaties met vrienden/familie/collega's en/of andere belangrijke bekenden verbroken en bent $u$ deze weer aangegaan? (zowel het uitmaken als aangaan worden gescoord)

2.8 Bent $u$ de afgelopen 3 maanden een of meerdere nieuwe relaties aangegaan met vrienden/familie/collega's en/of andere belangrijke bekenden en/of heeft $\mathrm{u}$ die beëindigd? (zowel het verbreken als het aangaan scoren) 


\section{Zelfbeeld}

Zelfidentiteit is een stabiel zelfgevoel dat zorgt voor eenheid van persoonlijkheid over tijd. Het type identiteitsstoornis dat kenmerkend is voor de Borderline Persoonlijkheidsstoornis bestaat uit extreme verschuivingen in het zelfbeeld van de persoon (wie ben ik), die zich manifesteren in plotselinge veranderingen wat betreft baan, carrière doelen, sexuele oriëntatie, persoonlijke waarden, vrienden en het fundamentele gevoel wat iemand over zichzelf heeft (bv. als goed of slecht). Dit item moet alleen gescoord worden als de identiteitsstoornis niet past bij de ontwikkelingsleeftijd van de persoon (d.i. normale volwassen identiteitsverschuivingen moeten hier niet toe gerekend worden).

3.1 Bent $\mathrm{u}$ de afgelopen 3 maanden in uiteenlopende situaties of met verschillende mensen zo anders geweest dat $u$ zich niet steeds als dezelfde persoon gedroeg en dat u niet meer wist wie $u$ eigenlijk was?
0 . afwezig
1. twijfelachtig / enige ondersteuning
2. waarschijnlijk niet weten wie hij/zij is, maar niet zo duidelijk omschreven
3. (vrij) duidelijk niet weten wie hij/zij is, maar niet zo overheersend
4. overheersend, duidelijk en welomschreven niet weten wie hij/zij is

3.2 Is het de afgelopen 3 maanden voorgekomen dat het idee, over wie u bent, sterk veranderde?
0 . afwezig
1. twijfelachtig / enige ondersteuning
2. waarschijnlijke instabiliteit van zelfidentiteit
3. (vrij) duidelijke instabiliteit van zelfidentiteit
4. duidelijk en overheersende instabiliteit van zelfidentiteit

3.3 Is het de afgelopen 3 maanden voorgekomen dat het gevoel, of u een goed of slecht mens bent, sterk veranderde?
0. afwezig
1. twijfelachtig / enige ondersteuning
2. waarschijnlijke instabiliteit van zelfgevoel
3. (vrij) duidelijke instabiliteit van zelfgevoel
4. duidelijk en overheersende instabiliteit van zelfgevoel

3.4 Wat zijn de afgelopen 3 maanden uw doelen op lange termijn in het leven geweest? Bv. welke opleiding, werk en/of carrière u zou willen of wensen ? 
Zijn deze doelen de afgelopen 3 maanden veranderd? (cliënten die in behandeling zijn zeggen vaak dat hun enige doel is beter worden en/of de therapie goed afmaken, dit wordt gescoord als vermijding)

0 . afwezig

1. twijfelachtig / enige ondersteuning

2. waarschijnlijke instabiliteit van lange termijn doelen/waarschijnlijke vermijding stil te staan bij lange termijn doelen

3. (vrij) duidelijke instabiliteit van lange termijn doelen, maar niet zo overheersend / (vrij) duidelijke vermijding stil te staan bij lange termijn doelen, maar niet zo overheersend

4. duidelijke en overheersende instabiliteit van lange termijn doelen / duidelijke en overheersende vermijding stil te staan bij lange termijn doelen

3.5 Bent u de afgelopen 3 maanden veranderd in uw opvatting over wat moreel goed en fout is? (... in uw opvatting over uw normen en waarden/ wat je wel en wat je niet kunt maken/ wat goed en slecht is). Doorvragen naar intensiteit/richting/frequentie van veranderingen)

0 . afwezig

1. twijfelachtig / enige ondersteuning

2. waarschijnlijke instabiliteit van morele waarden

3. (vrij) duidelijke instabiliteit van morele waarden, maar niet zo overheersend

4. duidelijke en overheersende instabiliteit van morele waarden

3.6 Heeft $\mathrm{u}$ er de afgelopen 3 maanden moeite mee gehad om te bepalen wat belangrijk is in uw leven? Zijn hier belangrijke veranderingen in geweest?

0. afwezig

1. twijfelachtig / enige ondersteuning

2. waarschijnlijke instabiliteit van persoonlijke waarden/ waarschijnlijke vermijding

3. (vrij) duidelijke instabiliteit van persoonlijke waarden, maar niet zo overheersend/ duidelijke vermijding, maar niet zo overheersend

4. duidelijke en overheersende instabiliteit van persoonlijke waarden/ duidelijke en overheersende vermijding

3.7 Heeft $\mathrm{u}$ er de afgelopen 3 maanden moeite mee gehad te bepalen wat voor soort vrienden $\mathrm{u}$ zou willen hebben? Veranderden het soort vrienden wat $\mathrm{u}$ had vaak? (sommige mensen hebben geen vrienden, dit wordt gescoord als vermijding)

0. afwezig

1. twijfelachtig / enige ondersteuning

2. waarschijnlijke instabiliteit m.b.t.. vrienden / waarschijnlijke vermijding m.b.t. vrienden 
3. (vrij) duidelijke instabiliteit m.b.t. vrienden, maar niet zo overheersend / duidelijke vermijding m.b.t. vrienden, maar niet zo overheersend

4. duidelijke en overheersende instabiliteit m.b.t. vrienden / duidelijke en overheersende vermijding m.b.t. vrienden

3.8 Heeft u er de afgelopen 3 maanden weleens aan getwijfeld of $u$ een seksuele relatie wilt met mannen óf met vrouwen? Na het antwoord: Hoe vaak is dit veranderd de afgelopen 3 maanden? (stabiele biseksuele (e.a.) oriëntatie wordt gescoord als 0 )
0. afwezig
1. zelden
2. heeft waarschijnlijke twijfels m.b.t. de sexuele oriëntatie
3. heeft vrij duidelijke twijfels m.b.t. de sexuele oriëntatie
4. heeft ernstige twijfels m.b.t. de sexuele oriëntatie 


\section{Impulsiviteit}

Hieronder volgen een paar voorbeelden van dingen die mensen impulsief kunnen doen, dus dingen waarvan $u$ later dacht dat $u$ ze beter niet had kunnen doen of dingen die $u$ of uw omgeving in de problemen brachten of hadden kunnen brengen. (het gaat niet om gedrag dat als primair doel heeft jezelf of anderen te beschadigen, het gaat om gedrag dat als primair doel heeft negatieve gevoelens uit te schakelen en/of als primair doel heeft positieve gevoelens te bewerkstelligen)

Het centrale kenmerk van dit criterium is het onvermogen van de persoon om controle uit te oefenen over zijn / haar impulsen, waardoor hij / zij betrokken raakt bij gedrag dat bevredigend is op de korte termijn, maar op de lange termijn mogelijk schadelijk is. De gedragingen die hieronder genoemd worden zijn voorbeelden, zij beslaan niet het volledige bereik.

0. nooit

1. $1 \times$ p. 3 maanden

2. $2 \times$ p. 3 maanden

3. $3 \times$ p. 3 maanden $/ 1 \times$ p. maand

4. 4 à $5 \times$ p. 3 maanden $/ 1 \times$ p. 3 weken

5. 6 à $7 \times$ p. 3 maanden $/ 1 \times$ p. 2 weken
6. 8 à $10 \times$ p. 3 maanden $/ 2 \times$ p. 3 weken

7. $1 \times$ p. week/11 à $15 \times$ p. 3 maanden

8. meerdere malen p. week maar minder dan de helft van de week

9. meer dan de helft van de week/bijna elke dag 10. dagelijks

4.1 Onverantwoordelijk geld uitgeven en/of meer geld uitgeven dan u eigenlijk kan uitgeven. (Bv. gokken, dingen impulsief kopen, veel en lang telefoneren.)

4.2 Seks met mensen die u niet of nauwelijks kende.

4.3 Onveilige seks. (dat is seks zonder rekening te houden met mogelijke zelfbeschadigende gevolgen en/of zwangerschap)

4.4 Teveel alcohol gebruiken of alcohol op verkeerde momenten. (Bij alcoho/verslaafden het vaste gebruik noteren onder $4.4 \mathrm{~A}$, alles wat extra wordt gebruikt scoren onder 4.4)

4.5 Teveel soft drugs gebruiken of soft drugs op de verkeerde momenten. (Bij drugverslaafden het vaste gebruik noteren onder $4.5 \mathrm{~A}$, alles wat extra wordt gebruikt scoren onder 4.5)

4.6 Inname van pillen. (niet met als doel zelfdoding, maar met als doel een roes te bewerkstelligen) 
4.7 Harddrugs gebruiken. (bij drugverslaafden het vaste gebruik noteren onder 4.7A, alles wat extra wordt gebruikt scoren onder 4.7)

4.8 Eetbuien. (alle eetbuien worden gescoord, dus met en zonder controleverlies, met en zonder planning etc.)

4.9 Roekeloos deelnemen aan het verkeer. (bv. te hard rijden of rijden onder invloed) (mag niet door dissociatie veroorzaakt worden)

4.10 Het plegen van diefstallen. (met welke intentie? Het moet gaan om het verkrijgen van een goed gevoel of het wegdrukken van een slecht gevoel, niet om zelfverrijking)

4.11 Andere zaken die $u$ impulsief heeft gedaan en die $u$ in moeilijkheden hadden kunnen brengen of u gebracht hebben? (bv. afspraken afzeggen/ niet nakomen, opgeven voor cursus/opleiding, reizen boeken) (niet automutilatie of suïcidaal gedrag, antwoord noteren op scoreformulier) 


\section{Parasuïcide}

Deze vragen gaan erover of $u$ in de afgelopen 3 maanden heeft getracht $u$ zelf pijn te doen of te verwonden. (niet scoren als het doel zelfdoding is)

\begin{tabular}{|c|c|c|}
\hline 0. nooit & 6. & 8 à 10 x p. 3 maanden $/ 2 \times$ p. 3 weken \\
\hline 1. $1 \times$ p. 3 maanden & 7. & $1 \times$ p. week $/ 11$ à $15 \times$ p. 3 maanden \\
\hline 2. $2 \times$ p. 3 maanden & 8. & meerdere malen $\mathrm{p}$. week maar minder dan de \\
\hline 3. $3 \times$ p. 3 maanden $/ 1 \times$ p. maand & & helft van de week \\
\hline 4. 4 à $5 \times$ p. 3 maanden $/ 1 \times$ p. 3 weken & 9. & meer dan de helft van de week/bijna elke dag \\
\hline 5. 6 à $7 \times$ p. 3 maanden $/ 1 \times$ p. 2 weken & 10. & Dagelijks \\
\hline
\end{tabular}

Automutilatie (met zelfbeschadiging als onmiddellijk gevolg,

d.i. weefselschade of fysieke pijn), zonder enige suicidale bedoeling.

5.1 Hoe vaak heeft $u$ de afgelopen 3 maanden u zelf opzettelijk geslagen of met uw hoofd, vuist knokkels of ander lichaamsdeel ergens tegenaan geslagen of geschuurd?

Of met uw vuist en/of ander lichaamsdeel een raam ingeslagen?

5.2 U zelf gekrabd of geknepen?

5.3 U zelf gebeten? (wat normaal gesproken pijn doet, dus niet nagelbijten)

5.4 Uw haren uitgetrokken? (kunnen ook wenkbrauwen/wimpers zijn)

5.5 U zelf gesneden en/of gekrast?

5.6 U zelf gebrand (sigaret, strijkijzer)?

5.7 Naalden e.d. in uw lichaam gestoken?

5.8 $U$ zelf op een andere manier opzettelijk beschadigd? (bv. scherpe voorwerpen inslikken, gevaarlijke stoffen innemen, het inbrengen van scherpe/gevaarlijke voorwerpen in lichaamsopeningen zoals vagina, penis, oren e.d., goed noteren op scoreformulier) 


\section{Sü̈cide(plannen/pogingen)}

5.9 Hoe vaak heeft u de afgelopen 3 maanden u zelf willen doden?

5.10 Hoe vaak heeft $\mathrm{u}$ de afgelopen 3 maanden tegen anderen gezegd dat $\mathrm{u}$ zichzelf wilde doden? (dit wordt niet gescoord als het gaat om passieve suïcidale ideevorming bv. tegen anderen zeggen: "ik wou dat ik dood was")

5.11 Hoe vaak heeft $u$ de afgelopen 3 maanden plannen gemaakt om u zelf te doden? (wanneer deze plannen uitmonden in stappen, scoren onder 5.12)

5.12 Hoe vaak heeft $\mathrm{u}$ de afgelopen 3 maanden stappen ondernomen om $\mathrm{u}$ zelf te doden? (wanneer deze stappen uitmonden in een poging, scoren onder 5.13)

5.13 Hoe vaak heeft $u$ de afgelopen 3 maanden een poging gedaan $u$ zelf te doden? 


\section{Stemmingswisselingen}

Ik wil u nu vragen naar stemmingswisselingen. Het gaat daarbij om opvallende wisselingen naar een sombere, prikkelbare, angstige, wanhopige en/of boze stemming.

Affectieve instabiliteit heeft betrekking op de wisselende, instabiele kwaliteit van de stemming van een persoon. Hoewel de stemmingsverandering vaak abrupt is, is een plotseling begin van de stemmingsverandering niet vereist. In plaats daarvan specificeert dit criterium frequente stemmingswisselingen, die weliswaar sterk zijn, maar slechts van relatief korte duur (eerder uren dan dagen of weken).

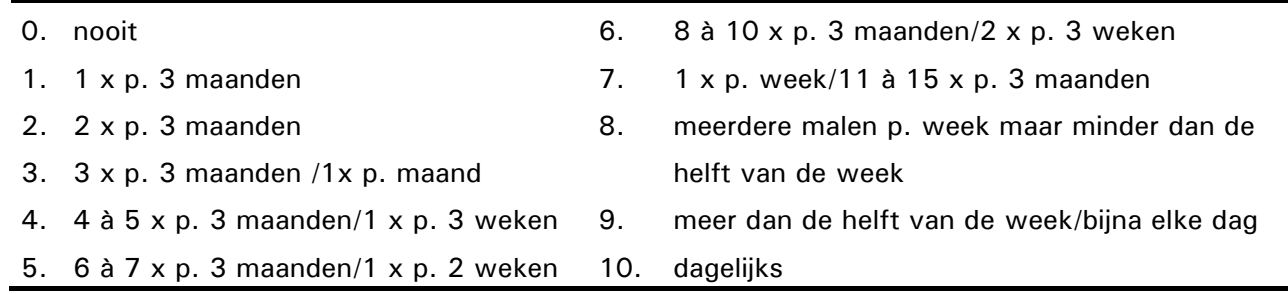

6.1 Kent u dergelijke stemmingswisselingen naar een sombere stemming? Hoe vaak zijn deze de laatste 3 maanden voorgekomen? (Niet door AS-I diagnose: uitvragen!/check ook SCID-I)

6.2 En naar een prikkelbare en/of geïrriteerde stemming? (Niet door AS-I)

6.3 En naar een angstige stemming? (Niet door AS-I)

6.4 En naar een wanhopige stemming? (Niet door AS-I)

6.5 En naar een boze stemming? (Niet door AS-I)

(bij twijfel over AS-I invloed wel scoren maar dit aantekenen en overleggen met onderzoeker) 


\section{Leegte}

De chronische gevoelens van leegte worden vaak geassocieerd met gevoelens van verveling, eenzaamheid, waardeloosheid, of gevoelens die "niet te definiëren" zijn.

0. nooit

1. $1 \times$ p. 3 maanden

2. $2 \times$ p. 3 maanden

3. $3 \times$ p. 3 maanden $/ 1 \times$ p. maand

4. 4 à $5 \times$ p. 3 maanden $/ 1 \times$ p. 3 weken

5. 6 à $7 \times$ p. 3 maanden $/ 1 \times$ p. 2 weken

\author{
6. 8 à $10 \times$ p. 3 maanden $/ 2 \times$ p. 3 weken \\ 7. $1 \times \mathrm{p}$. week/11 à $15 \times \mathrm{p} .3$ maanden \\ 8. meerdere malen p. week maar minder dan de \\ helft van de week \\ 9. meer dan de helft van de week/bijna elke dag \\ 10. dagelijks
}

7.1 Heeft u zich de afgelopen 3 maanden weleens verveeld of leeg gevoeld van binnen?

(Het gaat hier om gevoelens van leegte of verveling die spanningen geven of tot onaangepast gedrag leiden. Onder onaangepast gedrag wordt ook verstaan dat het normale of gewenste gedrag negatief wordt beïnvloed, bijv. tot niets komen terwijl iets anders gewenst of noodzakelijk was.)

7.2 Hoe vaak kwam u de afgelopen 3 maanden, als gevolg van deze gevoelens van leegte en verveling, tot niets terwijl u eigenlijk wat had willen doen? (bv. in bed liggen i.p.v. boodschappen doen)

7.3 Hoe vaak kwam het de afgelopen 3 maanden voor dat $u$, als gevolg van deze gevoelens van leegte en verveling, dingen ging doen, terwijl u eigenlijk iets anders had willen doen. (bv. uitgaan i.p.v. werken, ook alcohol en drugsgebruik vallen hieronder)

7.4 Hoe vaak is het de afgelopen 3 maanden voorgekomen dat u geen moment rust kon nemen? (bv. schoonmaken of ijsberen, dit wordt beschouwd als vermijding van rust nemen om gevoelens van leegte uit de weg te gaan) 


\section{Woedeuitbarstingen}

De volgende vragen gaan over driftbuien. Gebeurt het wel eens dat $u$ heel kwaad bent en/of woedeuitbarstingen heeft ?

Ongepaste woede verwijst naar de intensiteit van de woede van een persoon, deze staat niet in verhouding tot de oorzaak van de woede. Het tonen van extreem fysiek (gewelddadig) gedrag, zoals het slaan van mensen of het gooien van dingen kan wijzen op controleverlies m.b.t. woede. De woede wordt vaak geuit in de context van een daadwerkelijk of ervaren gebrek aan zorg / aandacht, verlies of verwaarlozing.

\begin{tabular}{|c|c|c|}
\hline 0. nooit & 6. & 8 à $10 \times$ p. 3 maanden $/ 2 \times$ p. 3 weken \\
\hline 1. $1 \times$ p. 3 maanden & 7. & $1 \times \mathrm{p}$. week $/ 11$ à $15 \times \mathrm{p} .3$ maanden \\
\hline 2. $2 \times$ p. 3 maanden & 8. & meerdere malen p. week maar minder dan de \\
\hline 3. $3 \times$ p. 3 maanden $/ 1 \times$ p. maand & & helft van de week \\
\hline 4. 4 à $5 \times$ p. 3 maanden $/ 1 \times$ p. 3 weken & 9. & meer dan de helft van de week/bijna elke dag \\
\hline 5. 6 à $7 \times$ p. 3 maanden $/ 1 \times$ p. 2 weken & 10. & dagelijks \\
\hline
\end{tabular}

8.1 Hoe vaak is dat de afgelopen 3 maanden gebeurd?

Kunt $\mathrm{u}$ aangeven hoe vaak $\mathrm{u}$ de afgelopen 3 maanden...

8.2 ... cynisch en/of sarcastisch reageerde op andere mensen. (bijtend, spottend)

8.3 ... gescholden, geschreeuwd en/of met deuren heeft geslaan. (verbaal)

8.4 ... zo kwaad was dat u niet meer aanspreekbaar was, dat u niet meer voor rede vatbaar was. (driftbuien)

8.5 ... met dingen heeft gegooid, gebroken heeft etc..

8.6 ... anderen aangevallen heeft. (fysiek) 


\section{Dissociatie en paranoïde ideeën}

Sommige mensen reageren sterk op stressvolle gebeurtenissen. In hoeverre heeft $u$ de afgelopen 3 maanden tijdens stressvolle gebeurtenissen last gehad van 1 van de volgende verschijnselen:

Gedurende periodes van stress, ontwikkelen sommige mensen met Borderline Persoonlijkheidsstoornis paranoïde of dissociatieve symptomen, van voorbijgaande aard, die zelden ernstig genoeg zijn om een additionele diagnose te stellen (d.i. psychotische stoornissen). De stressor is vaak daadwerkelijke, vermeende of geanticipeerde onthouding van zorg/aandacht van een zorgverlener (bv. partner, ouder, therapeut). In dergelijke situaties, kan daadwerkelijke of vermeende terugkeer van zorg / aandacht van de zorgverlener resulteren in een remissie van de symptomen. De dissociatieve symptomen bestaan uit periodes van dissociatieve amnesie (soms tot uiting komend doordat de persoon het gevoel heeft de "tijd" kwijt te zijn), depersonalisatie (d.i. het gevoel van zichzelf verwijderd of vervreemd te zijn) of derealisatie (d.i. het gevoel dat de externe wereld onwerkelijk of vreemd is). Deze periodes duren gewoonlijk enkele minuten of uren.

\begin{tabular}{|c|c|c|}
\hline 0. nooit & 6. & 8 à 10 x p. 3 maanden $/ 2 \times$ p. 3 weken \\
\hline 1. 1 x p. 3 maanden & 7. & $1 \times$ p. week/11 à $15 \times$ p. 3 maanden \\
\hline 2. $2 \times$ p. 3 maanden & 8. & meerdere malen $\mathrm{p}$. week maar minder dan de \\
\hline 3. $3 \times$ p. 3 maanden $/ 1 \times$ p. maand & & helft van de week \\
\hline 4. 4 à $5 \times$ p. 3 maanden $/ 1 \times$ p. 3 weken & 9. & meer dan de helft van de week/bijna elke dag \\
\hline 5. 6 à $7 \times$ p. 3 maanden $/ 1 \times$ p. 2 weken & 10. & dagelijks \\
\hline
\end{tabular}

Dissociatie: depersonalisatie (9.1), derealisatie (9.2), bewustzijn (9.3), geheugen (9.4 en 9.5)

9.1 Dat u zich niet meer $u$ zelf voelde, alsof $u$ buiten $u$ zelf stond, of $u$ zelf ervaarde alsof in een film of in een droom?

(nb.... zelf = zowel lichaam als geest) (niet door drugs)

9.2 Dat $u$ de wereld om $u$ heen geheel anders waarnam, of geheel anders ervaarde, zodat deze u heel vreemd of onwerkelijk voorkwam? (bv. andere mensen lijken onbekend of 'robots' te zijn) (niet door drugs)

9.3 Dat u niet meer wist wat u deed of waar u zich bevond? (niet door drugs) 
9.4 Dat u voor u bekende mensen en/of voorwerpen plotseling niet meer herkende?

9.5 Dat u zich belangrijke dingen helemaal niet meer kon herinneren? Iniet door drugs) (klinisch beoordelen of iets belangrijk is, dus niet altijd vanuit de beleving van cliënt)

\section{Paranoïde ideeën}

9.6 Er last van gehad dat $\mathrm{u}$ erg achterdochtig was of anderen wantrouwde? (niet door drugs) (het gaat om een idee)

9.7 Ervan overtuigd was dat anderen het op u gemunt hadden, dat $\mathrm{u}$ achtervolgd werd? (niet door drugs) (het gaat om een tijdelijke waan)

9.8 Ervan overtuigd was dat anderen u oneerlijk behandeld hadden? (niet door drugs) (het gaat om een tijdelijke waan) 


\section{The Borderline Personality Disorder Checklist}

KLACHTENLIJST - 47

ARNOUD ARNTZ, LAURA DREESSEN

\& JOSEPHINE GIESEN-BLOO

Universiteit Maastricht

Departement medische, klinische en experimentele psychologie

Postbus 616

6200 MD Maastricht

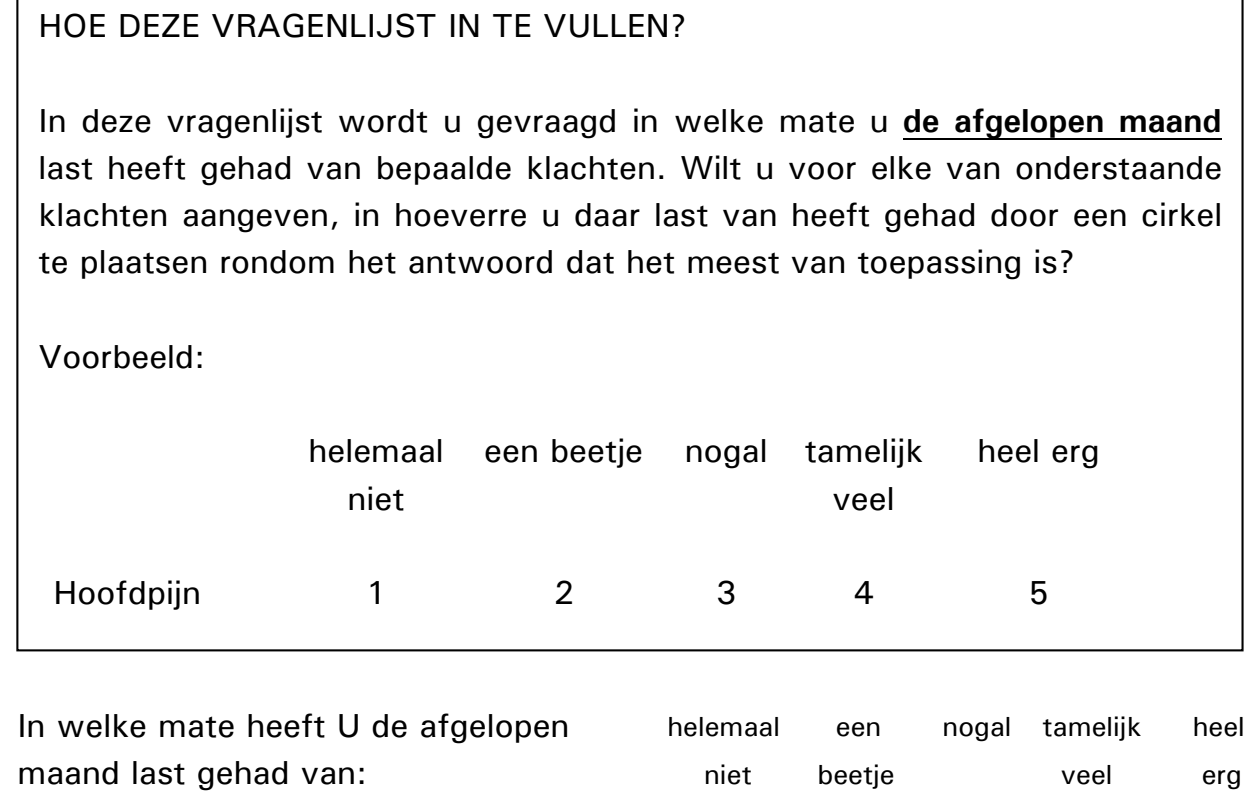

In welke mate heeft $U$ de afgelopen helemaal een nogal tamelijk heel maand last gehad van:

niet beetje veel erg

1. Impulsief teveel geld uitgeven,

$\begin{array}{llllll}\text { wat eigenlijk niet kan } & 1 & 2 & 3 & 4 & 5\end{array}$

$\begin{array}{lllllll}\text { 2. Snelle wisselingen in stemming } & 1 & 2 & 3 & 4 & 5\end{array}$

$\begin{array}{llllll}\text { 3. Woedeaanvallen } & 1 & 2 & 3 & 4 & 5\end{array}$ 
In welke mate heeft $U$ de afgelopen maand last gehad van:

4. Je niet meer jezelf voelen, alsof je buiten jezelf staat, of jezelf ervaart als in een film of een droom (niet door drugs)

5. Anderen slaan of met dingen gooien naar anderen

6. Jezelf opzettelijk verwonden (snijden, prikken, slaan, branden)

7. Niet weten of je je eigenlijk tot mannen of tot vrouwen aangetrokken voelt

8. Gokken

9. De neiging jezelf te doden

10. Onzekerheid over wie je eigenlijk bent

11. Je verveeld of leeg van binnen voelen

12. Teveel drinken

13. Angst dat anderen je verlaten

14. Zo anders zijn in verschillende situaties of bij verschillende mensen dat je niet meer weet wie je bent

15. Onzekerheid over hoe je leven eruit moet zien

$\begin{array}{ccccc}\text { helemaal } & \text { een } & \text { nogal } & \text { tamelijk } & \text { heel } \\ \text { niet } & \text { beetje } & & \text { veel } & \text { erg }\end{array}$

$\begin{array}{lllll}1 & 2 & 3 & 4 & 5\end{array}$


In welke mate heeft $U$ de afgelopen maand last gehad van:

16. Ervan overtuigd zijn dat anderen je oneerlijk behandelen

17. Drugsgebruik

18. Sterke veranderingen van gevoelens voor andere mensen

19. Wantrouwen in andere mensen

20. De slechte kanten van jezelf niet durven onderkennen

21. Het idee dat als anderen je werkelijk leren kennen, ze je zullen afwijzen

22. Roekeloos auto/motor/(brom-) fiets rijden

$\begin{array}{ccccc}\text { helemaal } & \text { een } & \text { nogal } & \text { tamelijk } & \text { heel } \\ \text { niet } & \text { beetje } & & \text { veel } & \text { erg }\end{array}$

$\begin{array}{lllll}1 & 2 & 3 & 4 & 5\end{array}$

$\begin{array}{lllll}1 & 2 & 3 & 4 & 5\end{array}$

$\begin{array}{lllll}1 & 2 & 3 & 4 & 5\end{array}$

$\begin{array}{lllll}1 & 2 & 3 & 4 & 5\end{array}$

$\begin{array}{lllll}1 & 2 & 3 & 4 & 5\end{array}$

$\begin{array}{lllll}1 & 2 & 3 & 4 & 5\end{array}$

$\begin{array}{lllll}1 & 2 & 3 & 4 & 5\end{array}$

23. De wereld om je heen geheel anders waarnemen, of geheel anders ervaren, zodat deze je heel vreemd of onwerkelijk voorkomt (bv. andere mensen lijken onbekend of 'robots' te zijn) (niet door drugs)

$\begin{array}{llll}2 & 3 & 4 & 5\end{array}$

24. De neiging levensgevaarlijke dingen te doen (b.v. in het verkeer)

$\begin{array}{lllll}1 & 2 & 3 & 4 & 5 \\ 1 & 2 & 3 & 4 & 5 \\ 1 & 2 & 3 & 4 & 5\end{array}$

26. Proberen jezelf te doden

27. Buiten jezelf raken omdat je meent dat iemand die belangrijk voor je is je gaat verlaten

$\begin{array}{lllll}1 & 2 & 3 & 4 & 5\end{array}$


In welke mate heeft $U$ de afgelopen maand last gehad van:

28. Anderen dreigen jezelf te verwonden of te doden

29. Vreetpartijen

30. Jezelf eigenlijk een slecht en onacceptabel persoon vinden

31. Ervan overtuigd zijn dat anderen het op je gemunt hebben

(dat je achtervolgd wordt)

32. Niet weten wat voor vrienden of geliefden je wilt hebben

33. Gevoelens die onacceptabel voor je zijn

34. Niet weten wat eigenlijk belangrijk voor je is

35. Winkeldiefstallen

36. Plotselinge angsten, depressies of prikkelbaarheid

37. Zo boos worden dat je de controle over jezelf verliest en dan dingen kapot maakt

38. Je belangrijke dingen helemaal niet meer kunnen herinneren (niet door drugs)

39. Erg achterdochtig zijn

$\begin{array}{ccccc}\text { helemaal } & \text { een } & \text { nogal } & \text { tamelijk } & \text { heel } \\ \text { niet } & \text { beetje } & & \text { veel } & \text { erg }\end{array}$

$\begin{array}{lllll}1 & 2 & 3 & 4 & 5\end{array}$

$\begin{array}{lllll}1 & 2 & 3 & 4 & 5\end{array}$

$\begin{array}{lllll}1 & 2 & 3 & 4 & 5\end{array}$

$\begin{array}{lllll}1 & 2 & 3 & 4 & 5\end{array}$

$\begin{array}{lllll}1 & 2 & 3 & 4 & 5\end{array}$

$\begin{array}{lllll}1 & 2 & 3 & 4 & 5\end{array}$

$\begin{array}{lllll}1 & 2 & 3 & 4 & 5\end{array}$

$\begin{array}{lllll}1 & 2 & 3 & 4 & 5\end{array}$

$\begin{array}{lllll}1 & 2 & 3 & 4 & 5\end{array}$

$\begin{array}{lllll}1 & 2 & 3 & 4 & 5\end{array}$

$\begin{array}{lllll}1 & 2 & 3 & 4 & 5\end{array}$


In welke mate heeft $U$ de afgelopen maand last gehad van:

40. Je verschrikkelijk teleurgesteld voelen door iemand die je eerst bewonderde of waar je van hield

41. Impulsief sexuele contacten aangaan waar je later spijt van had

42. Plotseling het vertrouwen in andere mensen verliezen

43. De overtuiging dat je niet in staat bent het leven alleen aan te kunnen

44. Jezelf, iedereen en de hele wereld haten

45. Krampachtig proberen te voorkomen dat anderen je in de steek laten

46. Onzekerheid over wat je echte normen en waarden zijn

47. Niet meer weten wat je gedaan hebt of waar je je bevindt (niet door drugs)

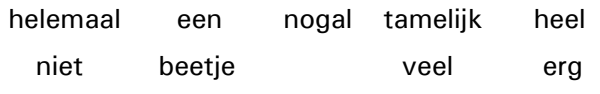

$\begin{array}{lllll}1 & 2 & 3 & 4 & 5\end{array}$

$\begin{array}{lllll}1 & 2 & 3 & 4 & 5\end{array}$

$\begin{array}{lllll}1 & 2 & 3 & 4 & 5\end{array}$

$\begin{array}{lllll}1 & 2 & 3 & 4 & 5\end{array}$

$\begin{array}{lllll}1 & 2 & 3 & 4 & 5\end{array}$



Dankwoord 


\section{Dank!}

Eindelijk is het dan af. Heel wat cliché's hebben me de afgelopen jaren hun gelijk getoond, zo ook dat achter ieder wetenschappelijk artikel de medewerking en ondersteuning van vele mensen schuilgaat. Alleen mijn naam staat echter op deze bundeling van zeven jaar intensief werken. Op deze plek wil ik daarom een aantal mensen bij naam noemen en bedanken, zonder hen zou dit proefschrift er niet zijn gekomen.

Allereerst dank ik mijn promotoren, de professoren Arnoud Arntz, Richard van Dyck en Philip Spinhoven, voor de mogelijkheid en het vertrouwen dat ik als landelijk coördinator/organisator en onderzoeker aan de borderline behandelstudie mocht gaan werken. Ik hoop dat deze thesis jullie namen eer aan doet.

Beste Arnoud, Als $1^{\mathrm{e}}$ promotor in Maastricht was je niet alleen mijn eerste aanspreekpunt in praktische zin, maar heb je ook het meeste invloed gehad op mij als onderzoeker. Ik heb groot respect en bewondering voor de passie en integriteit waarmee je zowel fundamenteel als klinisch toegepast wetenschappelijk onderzoek initieert en uitvoert (ook als het bijna onuitvoerbare projecten lijken te zijn), en daarnaast nog de link met de klinische praktijk behoudt. Je enthousiasme is aanstekelijk en inspirerend, het snelle nakijken van stukken werkt eveneens stimulerend! Ik heb ook veel geleerd van je brede kennis en je ondernemingszin om die kennis te blijven verbreden, dan denk ik bijvoorbeeld aan de 'spoedcursus' robuuste statistiek die volgde toen de data van de behandelstudie niet voldeden aan de assumpties voor parametrische statistiek. De vrijheid en zelfstandigheid waarin ik mocht werken vond ik niet altijd even gemakkelijk, maar maakten dat ik me eveneens breed heb kunnen ontwikkelen. Veel dank voor dit alles.

Beste Philip en Richard, veel dank voor de ondersteuning, betrokkenheid en inbreng vanuit Leiden en Amsterdam, zeker in de laatste fase van dit proefschrift. Op zich waren jullie, letterlijk en figuurlijk, meer op de achtergrond. Ik heb echter altijd het gevoel gehad, en dat is ook door de jaren heen gebleken, dat ik wel gelijk een beroep op jullie kon doen. Philip, ongelofelijk hoe snel jij emails weet te beantwoorden! Ik heb jullie bijdragen bijzonder gewaardeerd.

I would like to thank the members of the reading committee, Dr. David Bernstein, Prof. Dr. Wim van den Brink, Prof. Dr. Anita Jansen, Prof. Dr. John Livesley en Prof. Dr. Johan Vlaeyen for their willingness and efforts to review this thesis.

Oprechte dank ben ik verschuldigd aan alle mensen die de borderline behandelstudie mogelijk hebben gemaakt: 
De borderline cliënten, maar zeker ook de cliënten en controle personen die buiten de borderline behandelstudie hebben deelgenomen, die zoveel uren hebben meegewerkt aan interviews, vragenlijsten en andere testen waarin de voor hen meest moeilijke onderwerpen aan de orde waren. Dank voor jullie jarenlange medewerking!

De screeners en onderzoeksassistenten. Jullie vormden een cruciale schakel, daar waar de data werd verzameld, en dat was niet altijd vanzelfsprekend of gemakkelijk werken! Ik waardeer jullie inspanningen en doorzettingsvermogen zeer. Dank voor de plezierige samenwerking.

Ismay Kremers en Marjon Nadort, Leids/Haagse en Amsterdamse coördinator, dank voor de fijne samenwerking en voor het delen van zoveel meer. Het is inmiddels duidelijk geworden dat 'het delen van zoveel meer' ook na het borderline project blijft bestaan, daar ben ik blij om.

Thea van Asselt, kosteneffectiviteit onderzoeker. Onze samenwerking verliep eigenlijk vooral per telefoon of e-mail, verder bijkletsen gebeurde meer tijdens lunch- of koffie'dates'. Dank je wel daarvoor.

De SFT- en TFP-therapeuten. Ik ben me altijd bewust geweest dat jullie samenwerking in één onderzoek (vooralsnog) uitzonderlijk was. Vanaf de eerste landelijke trainings/supervisie bijeenkomsten heb ik het heel bijzonder gevonden, en me bevoorrecht gevoeld om zoveel van jullie werken van dichtbij te mogen zien, en ervan te mogen leren. Ik heb me zowel in de SFT als de TFP-groep welkom en thuis gevoeld. Dank voor dit al, evenals voor jullie inspanningen voor- en betrokkenheid bij het onderzoek.

Consultants Frank Yeomans and Jeffrey Young. I feel very privileged and honored that I could work with you. A sincere 'thank you' for everything your workshops/supervisions taught me on TFP and SFT, and for the conversations that contributed to my becoming a researcher with an eye for the clinical perspective.

In Maastricht was mijn standplaats het Departement Medische Klinische en Experimentele Psychologie, waarvan ik de volgende (oud-)collega's expliciet wil bedanken.

Statisticus Erik Schouten, dank voor je uitleg, advies en inspanningen, altijd op een kalme en rustige manier. Ik ben er heel wat wijzer van geworden! Rosanne Janssen, veel dank voor de (plezierige) ondersteuning wat betreft databases en het omzetten in, voor mij leesbare, SPSS-bestanden. Lindy Dullens, Annie Raven en Angela Verwey, dank jullie wel voor de administratieve en organisatorische ondersteuning, maar ook voor jullie persoonlijke betrokkenheid.

Mijn kamergenoten Simkje Sieswerda, Manon Vincken en Lotte Bamelis. Of jullie wilden of niet, jullie hebben veel van mijn wel en wee gedurende de afgelopen zeven jaar meegekregen. Dank dat ik soms zomaar kon blijven ratelen en zo 
dan de dingen kon ordenen. Lotte, jouw humor tijdens de pittige laatste loodjes gaf me het nodige, gezonde tegenwicht, en het heeft me geholpen productief te blijven!

Anneke van Brakel, Nicole Buck, Maaike Cima, Elizabeth Dorant, Iris Engelhard, Jill Lobbestael, Hanneke Oomkes, en Anoek Weertman: dank jullie wel voor de morele steun en afleiding, en de gesprekken die verder reikten dan de verschillende facetten van wetenschappelijk onderzoek. Mijn contact met een ieder van jullie eindigt gelukkig niet met de voltooiing van dit proefschrift!

Joke Opdenacker en Lieven Wachters, bedankt voor de tijd en energie die jullie, door middel van jullie afstudeerstages, in dit onderzoek hebben gestoken.

Mijn nieuwe collega's van F.P.C. "de Rooyse Wissel", Farid Chakhssi en Marjolein van Vorstenbos, wil ik van harte bedanken voor de geboden ruimte en belangstelling in de aller-, allerlaatste fase van dit proefschrift.

Mijn paranimfen en heel bijzondere vriendinnen, Karin Flobbe en Ina Leeuw. Ik ben intens blij dat twee mensen die me erg dierbaar zijn, en tijdens het hele promotietraject zo ontzettend belangrijk zijn geweest, dan ook op de grote dag naast me staan. Dank voor alles, en op naar meer ontspannen tijden.

Nolda van Asseldonk, Liesbeth Billy, Astrid Claessens, Katja Schmitz en Pascale Voermans. De gesprekken die ik de afgelopen jaren met ieder van jullie over, en naar aanleiding van mijn werk heb gevoerd, waren erg belangrijk voor me, en hebben me geholpen en gesteund bij het afmaken van dit proefschrift.

Al mijn andere vrienden en familie wil ik eveneens bedanken voor hun niet aflatende belangstelling en het begrip als ik het een tijd liet afweten in verband met mijn werk.

Rob Klein Goldewijk, wat vind ik het fijn dat jij de omslag hebt willen maken, en wat is het mooi geworden! Dank je wel. Marike Mulder, dank voor je spontane medewerking.

Mijn ouders. Als kind was het al vanzelfsprekend dat ik zou gaan studeren. Hoe bijzonder dat was, realiseerde ik me echter pas jaren later. Ook tijdens deze promotiejaren hebben jullie heel erg meegeleefd, veel dank daarvoor.

Mijn kinderen, Pieter, Loes en Geert. Eindelijk is dat boek van mamma dan af, en nu is het ook echt voorbij. En ook al hadden jullie het waarschijnlijk niet door; jullie waren (en zijn) mijn ultieme relativeringsbron, het gaat gewoon om ons vijven.

Mijn man, Peter. Het 'ondanks' voorbij, is het met name dankzij jou dat het me gelukt is te blijven doorgaan en dit proefschrift af te ronden. Dank je wel voor je liefde en energie. Wat hebben we toch intense jaren achter de rug, op naar de rust en het betere levensgenieten. 


\section{Curriculum vitae}




\section{Curriculum vitae}

Josephine Giesen-Bloo was born on August 6, 1971, in Nijmegen, the Netherlands. In 1990 she graduated from secondary school (Atheneum, Pax Christi College in Druten). Consequently, she studied Health Sciences at the Rijksuniversiteit Limburg (currently University Maastricht) and obtained her MSc in Mental Health Sciences in September 1994. From November 1994 to January 1996 she worked as a research fellow at the Department of Psychiatry at Mount Sinai Medical School in New York (USA) with Prof. Dr. David Greenberg. In New York, she and her husband Peter had three children: Pieter (1996), Loes (1997) and Geert (1998). When they returned to the Netherlands, she started working as a research fellow on the Dutch borderline treatment study with professors Arnoud Arntz (UM), Philip Spinhoven (Leiden University) and Richard van Dyck (VU Medical Center Amsterdam), based at the department of medical, clinical and experimental psychology (dmcep) at the University of Maastricht and the department of medical psychology of the Maastricht University Hospital. The present thesis is the result of these years (April 1999 to September 2005). Since October 2005 she is a researcher at forensic psychiatric center "De Rooyse Wissel" in Venray, and has an honorary appointment at the University of Maastricht (dmcep). 


\section{Papers}

International (peer-reviewed) journals

Giesen-Bloo, J. \& Arntz, A. (2005). World assumptions and the role of trauma in borderline personality disorder. Journal of Behavior Therapy and Experimental Psychiatry, 36, 197-208.

Giesen-Bloo, J., Wachters, L., Arntz, A. \& Schouten, E. (2006). Assessment of borderline personality disorder with the Borderline Personality Disorder Severity Index-IV: psychometric evaluation and dimensional structure. Submitted.

Giesen-Bloo, J., van Dyck, R., Spinhoven, Ph., van Tilburg, W., Dirksen, C., van Asselt, Th., Kremers, I., Nadort, M. \& Arntz, A. (2006). Outpatient psychotherapy for borderline personality disorder: a randomized trial of schema focused therapy versus transference focused therapy. Archives of General Psychiatry, 63, 649-58.

Giesen-Bloo, J., Arntz, A. \& Schouten, E. (2006). The borderline personality disorder Checklist: psychometric evaluation and factorial structure in clinical and nonclinical samples. Submitted.

Giesen-Bloo, J., Opdenacker, J., Spinhoven, Ph. \& Arntz, A. (2006). The defense style questionnaire - 48: psychometric evaluation and factorial structure in clinical and nonclinical samples. Submitted.

Spinhoven, Ph., Giesen-Bloo, J., van Dyck, R., Kooiman, K. \& Arntz, A. (2006). The therapeutic alliance in schema focused therapy and transference focused psychotherapy for borderline personality disorder. Submitted.

Spinhoven, Ph., van Dyck, R., Giesen-Bloo, J. \& Arntz, A. (2006). Can assessors and therapists predict the outcome of long-term psychotherapy in borderline personality disorder? Submitted.

Asselt, Th. van, Dirksen, C., Severens, J., Arntz, A., van Dyck, R., Spinhoven, Ph., Giesen-Bloo, J., Kremers, I. \& Nadort, M. (2006). Costeffectiveness of schema focused therapy and transference focused therapy for borderline personality disorder. Submitted.

\section{National (peer-reviewed) journals}

Giesen-Bloo, J. \& Arntz, A. (2000). Borderline persoonlijkheidsstoornis: een uitdaging voor behandeling. De Psycholoog, 35 (7/8), 317-324.

Giesen-Bloo, J. \& Arntz, A. (2000). Psychoanalytische psychotherapie en cognitieve gedragstherapie voor borderline persoonlijkheidsstoornis. Gedrag \& Gezondheid, 28, 288-295. 


\section{Book-section and book}

Giesen-Bloo, J. (2004). Poliklinische behandeling van borderline persoonlijkheidsstoornis: cognitieve schemagerichte gedragstherapie versus 'transference focused psychotherapy'. In: W.C.B. Hoenink, M.J. Rexwinkel \& W. Roelofsen (red.) Psychoanalytische psychotherapie vergelijkenderwijs (p.68-78). Assen: van Gorcum.

Giesen-Bloo, J. (2005). Leven met een borderline persoonlijkheidsstoornis. Houten: Bohn, Stafleu \& van Loghum.

\section{Other}

Giesen-Bloo, J. \& Arntz, A. (2000). Reactie op 'missie met omissies'. De Psycholoog, 35(12), 565.

Giesen-Bloo, J. (2004). Boeken-wijzer: Linehan, M.M. (2002) Cognitieve gedrags therapie voor borderline persoonlijkheidsstoornis: theorie en behandeling en Borderline persoonlijkheidsstoornis: handleiding voor training en therapie. Diagnostiek wijzer, 87-90.

\section{Conference Presentations}

Giesen-Bloo, J. Outpatient treatment of borderline personality disorder: analytical psychotherapy versus cognitive behaviour therapy. World Congress of Behavioral and Cognitive Therapies, Vancouver (Canada) July, 2001.

Giesen-Bloo, J. World assumptions and trauma in borderline personality disorder. European Association of Behavioral and Cognitive Therapies. Maastricht, September 2002.

Giesen-Bloo, J. Transference-focused psychotherapy for borderline personality disorder symposium, New York (USA), November 2002.

Giesen-Bloo, J., Poliklinische behandeling van borderline persoonlijkheidsstoornis: cognitieve schemagerichte gedragstherapie versus 'transference focused psychotherapy'. Voorlopige resultaten van een voortdurend onderzoek. Studiedag NVPP, Amersfoort Juni 2003.

Giesen-Bloo, J., Effectiveness of schema focused therapy versus transference focused psychotherapy. EPP symposium personality disorders - state of the art, Amsterdam, Mei 2004.

Giesen-Bloo, J., Psychodynamisch of cognitief? Nascholingsdag Psychotherapie voor persoonlijkheidsstoornissen, Benecke NI. Utrecht, Juni 2004.

Giesen-Bloo, J., Outpatient treatment of borderline personality disorder: schema focused therapy versus transference focused psychotherapy. European 
Association of Behavioral and Cognitive Therapies. Manchester (United Kingdom), September 2004.

Giesen-Bloo, J., Effectiviteit van schemagerichte cognitieve gedragstherapie en Transference focused psychotherapie voor borderline persoonlijkheidsstoornis. Studiedag Psychiater Expertise groep Persoonlijkheidsstoornissen, Amersfoort, Februari 2005.

Giesen-Bloo, J., Psychodynamisch of cognitief? Nascholingsdag Psychotherapie voor persoonlijkheidsstoornissen, Benecke NI. Utrecht, Maart 2005.

Giesen-Bloo, J., Arntz, A. Outpatient treatment of borderline personality disorder: schema focused therapy versus transference focused psychotherapy. First meeting of the International Society for Schema Therapy. Stockholm, April 2006.

Giesen-Bloo, J., Outpatient treatment of borderline personality disorder: schema focused therapy versus transference focused psychotherapy. International Association of Forensic Mental Health Services. Amsterdam, June 2006. 